\title{
PROSODY MODELLING USING MACHINE LEARNING TECHNIQUES FOR NEUTRAL AND EMOTIONAL SPEECH SYNTHESIS
}

A dissertation for the degree of

Doctor of Philosophy

By

\section{Alexandros Anastasios-Georgios Lazaridis}

Electrical and Computer Engineer

UNIVERSITY of PATRAS

POLYTECHNIC SCHOOL

DEPARTMENT OF ELECTRICAL AND COMPUTER ENGINEERING WIRE COMMUNICATIONS LABORATORY 


\title{
ПАNЕПI $\Sigma$ THMIO ПАТР $\Omega N$ \\ ПO $\Lambda$ YTEXNIKH $\Sigma X O \Lambda H$
}

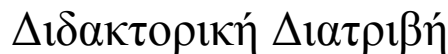 \\ $\tau \mathrm{OV}$
}

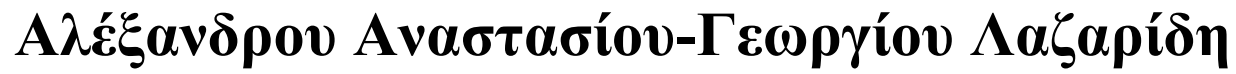

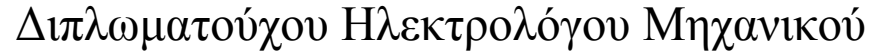

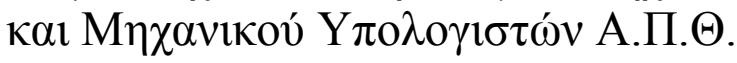

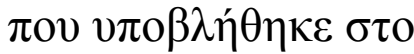

\section{TМНМА НАЕКТРОАОГ

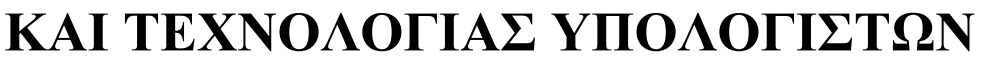

$\mu \varepsilon \tau i ́ \tau \lambda o$

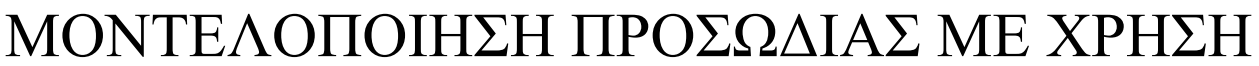 TEXNIK $\Omega$ N MHXANIKH $\Sigma$ MA $\Theta H \Sigma H \Sigma \Sigma T A$ $\Pi \Lambda A I \Sigma I A$ OY $\Delta$ ETEPH $\Sigma$ KAI $\Sigma$ YNAI $\Sigma \Theta H M A T I K H \Sigma$ $\Sigma$ YN $\Theta E T I K H \Sigma$ OMI $\Lambda$ IA $\Sigma$}




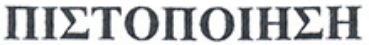

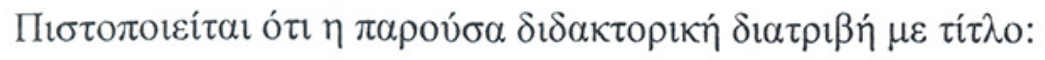

\section{MONTE $\Lambda$ OПOIH $\Sigma H$ ПPO $\Sigma \Omega \Delta I A \Sigma$ ME XPH $\Sigma H$ TEXNIK $\Omega N$ MHXANIKH $\Sigma$

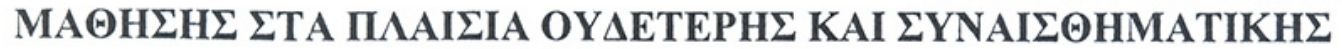

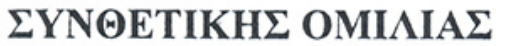

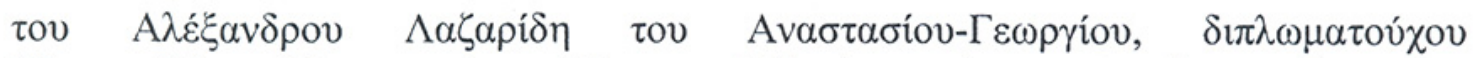

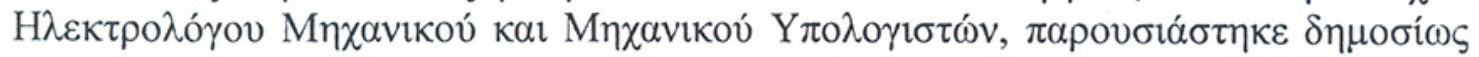

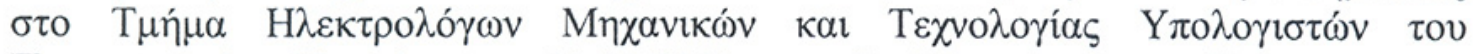

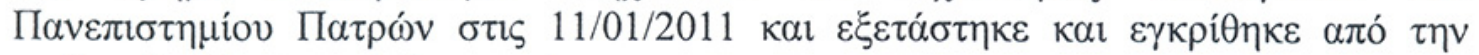

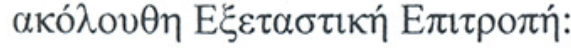

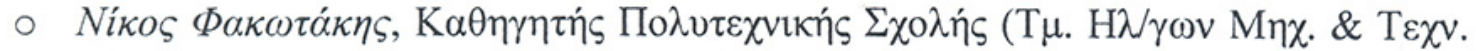

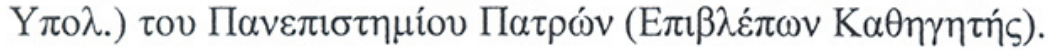

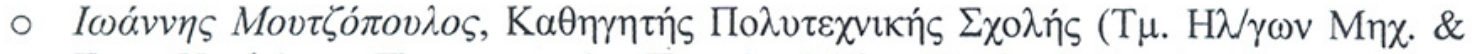

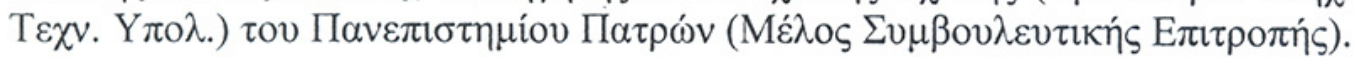

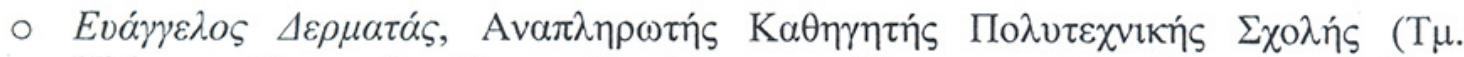

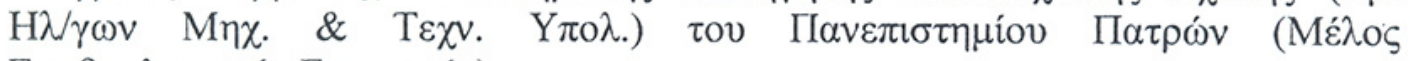

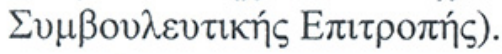

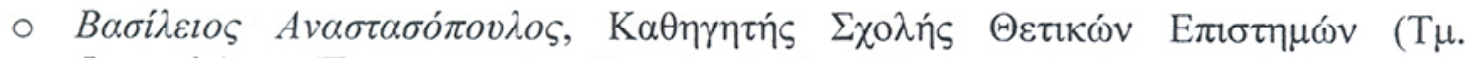

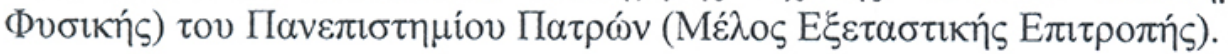

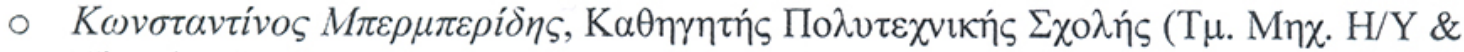

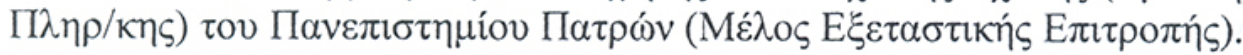

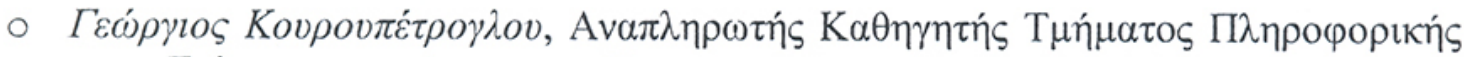

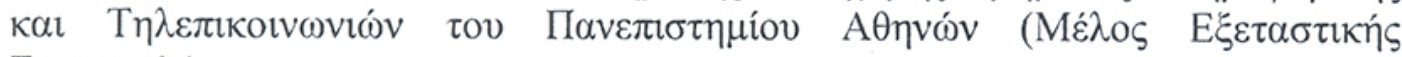

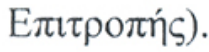

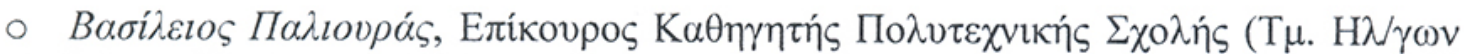

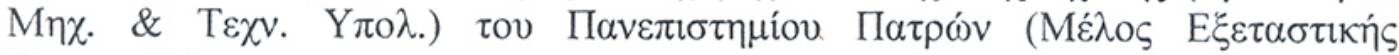

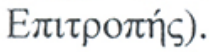

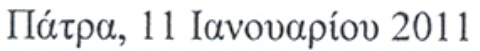
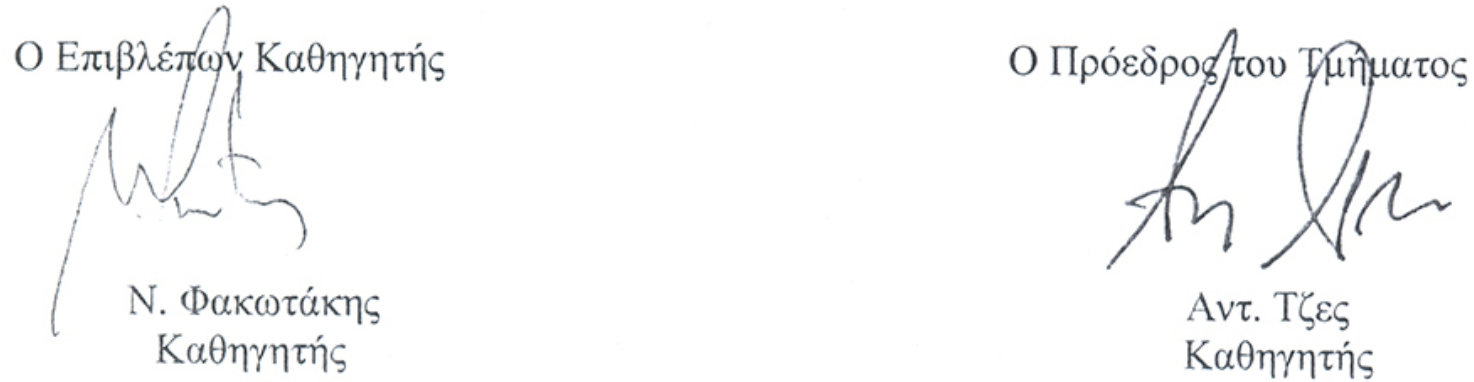

$\mathrm{A} v \tau . T \zeta \varepsilon \zeta$

$\mathrm{K} \alpha \theta \eta \gamma \eta \tau \dot{\zeta} \varsigma$ 


\section{Members of the Examination Committee}

- Nikos Fakotakis, Professor of the Electrical and Computer Engineering Department of the University of Patras, Director of the Telecommunications and Information Technology Division (Supervisor).

- John Mourjopoulos, Professor of the Electrical and Computer Engineering Department of the University of Patras (Member of the Advising Committee).

- Evaggelos Dermatas, Associate Professor of the Electrical and Computer Engineering Department of the University of Patras (Member of the Advising Committee).

- Vassilis Anastassopoulos, Professor of the Department of Physics of the University of Patras (Member of the Examination Committee).

- Kostas Berberidis, Professor of the Department of Computer Engineering and Informatics of the University of Patras (Member of the Examination Committee).

- Georgios Kouroupetroglou Associate Professor of the Department of Informatics and Telecommunications of the University of Athens (Member of the Examination Committee).

- Vassilis Paliouras, Assistant Professor of the Electrical and Computer Engineering Department of the University of Patras (Member of the Examination Committee). 
This work is dedicated to my parents, Tasos and Filio, and to my brothers, Nikos and Mitsos. 


\section{Acknowledgments}

This dissertation thesis took place in the Wire Communications Laboratory of Electrical and Computer Engineering Department of the University of Patras.

I would like to express my acknowledgments to the supervisor of my $\mathrm{PhD}$ study, the Director of the Wire Communications Laboratory, Prof. Nikos Fakotakis, for his valuable guidance. His comprehensive support and countenance made possible the successful completion of this work.

Furthermore, I would like to thank the members of the Advising Committee, Prof. John Mourjopoulos and Assoc.Prof. Evaggelos Dermatas for their guidance during my $\mathrm{PhD}$ study.

I would like also to express my thanks to Prof. George Kokkinakis for his valuable help.

Moreover, I would like to thank my friend Dr. Iosif Mporas for his valuable help and contribution to my study. Moreover I would like to thank my colleague Dr. Todor Ganchev for his valuable help to my study. Furthermore, I would like to thank all the rest of my colleagues of the Wire Communications Laboratory.

I would like to thank my second family, Assist.Prof. Thrasos Panidis family, for supporting me and helping me to adjust in Patras.

Finally, I would like to thank my family, my grandparents and all the rest of my family, for their precious help and support in every way in every step of my life and for making me the person I am today. I owe everything to them. 


\begin{abstract}
The dissertation is structured as follows:

In Chapter 1, a brief introduction to speech processing and consequently to speech synthesis is made. Furthermore, the basic concept of prosody is presented along with a brief introduction to machine learning and how it is applied in phone duration modelling. Finally, the Thesis contribution and structure are presented.

In Chapter 2, the design and development of a speech database for the Greek language is presented along with the development of two speech synthesis systems, a unit-selection corpus-based synthesis system and an HMM-based statistical parametric speech synthesis system, developed during this dissertation.

In Chapter 3, an introduction to prosody modelling is presented. Specifically, an overview is done concerning the phone duration modelling approaches and techniques which have been studied in the literature over the years. Furthermore, a description of the features which have been used in this task is done.

In Chapter 4, the three proposed approaches for phone duration modelling are presented. Specifically, the support vector regression (SVR) model, the fusion scheme based on the combination of the predictions of individual phone duration models and the two-stage phone duration modelling scheme with feature construction and feature vector extension are described in detail and evaluated.

In Chapter 5, phone duration modelling is studied in emotional speech. Feature selection techniques are applied in order to evaluate the features and select the most appropriate ones improving the phone duration modelling accuracy. Furthermore, the first proposed approach, SVR, is applied and evaluated in emotional speech.

In Chapter 6, perceptual evaluation tests are presented in order to evaluate the improvement of the proposed phone duration modelling schemes in terms of improvement of the naturalness of synthetic speech.

In Chapter 7, a summary of the Thesis is given along with the conclusions and the feature work concerning phone duration modelling in neutral and emotional speech.

The three proposed approaches were evaluated using two databases of different languages, one American-English and one Greek. The proposed approaches were compared to the state-of-the-art models in the phone duration modelling task.
\end{abstract}


The SVR model outperformed all the other individual models evaluated in this dissertation. Their ability to outperform all the other models is mainly based on their advantage of coping in a better way with high-dimensionality feature spaces in respect to the other models used in phone duration modelling, which makes them appropriate even for the case when the amount of the training data would be small respectively to the number of the feature set used.

The proposed fusion scheme, taking advantage of the observation that different prediction algorithms perform better in different conditions, when implemented with SVR (SVR-fusion), contributed to the improvement of the phone duration prediction accuracy over that of the best individual model (SVR). Furthermore the SVR-fusion model managed to reduce the outliers in respect to the best individual model (SVR).

Moreover, the proposed two-stage scheme using individual phone duration models as feature constructors in the first stage and feature vector extension (FVE) in the second stage, implemented with SVR (SVR-FVE), improved the prediction accuracy over the best individual predictor (SVR), and the SVR-fusion scheme and moreover managed to reduce the outliers in respect to the other two proposed schemes (SVR and SVR-fusion). The SVR two-stage scheme confirms in this way their advantage over all the other algorithms of coping well with high-dimensionality feature sets. The improved accuracy of phone duration modelling contributes to a better control of the prosody, and thus quality of synthetic speech.

Furthermore, in the study concerning the phone duration modelling in emotional speech and how feature selection techniques could improve the performance accuracy, the experimental results demonstrated that in four out of the five categories of emotional speech, i.e. Anger, Fear, Neutral and Sadness, regardless of the machine learning algorithm used for phone duration modelling, feature selection contributed to achieving a better phone duration prediction accuracy, when compared to the case without feature selection. Moreover the SVR algorithm was also evaluated on the phone duration modelling task in emotional speech, outperforming all the other models with and without feature selection in all the emotional categories. The SVR model with feature selection did not manage to outperform the SVR with no feature selection models in none of the emotional categories, showing that the SVR model manages to evaluate the features and use them in the best way without the need of feature selection algorithms. This shows that SVR copes well with small amount of data in respect to the feature set. 
Finally, perceptual tests were performed evaluating the impact of the proposed phone duration models to synthetic speech. The perceptual test for both the databases confirmed the results of objective tests showing the improvement achieved by the proposed models in the naturalness of synthesized speech. Finally, Wilcoxon test was performed, showing that the results of the MOS tests are statistical significant for all the proposed methods for both databases. 


\section{Пєрí $\lambda \psi \psi \eta$}

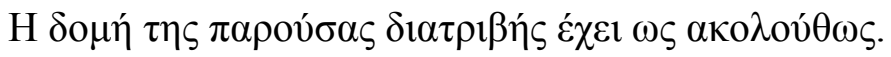

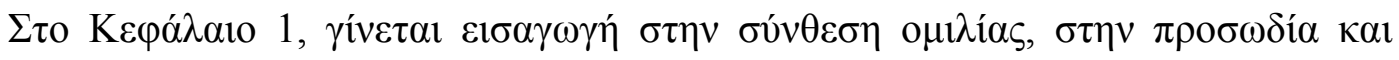

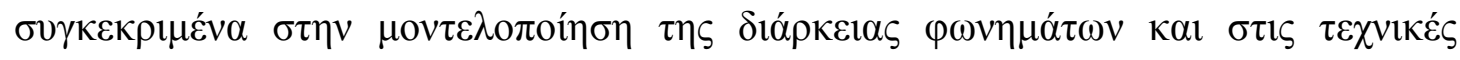

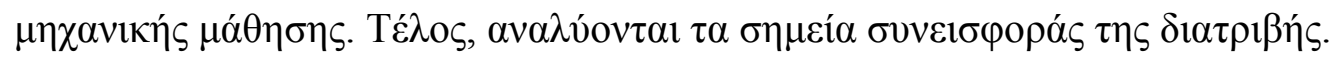

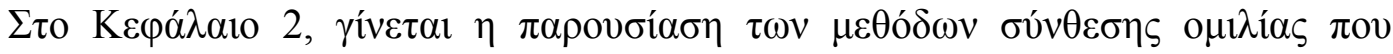

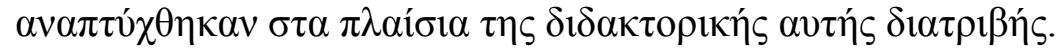

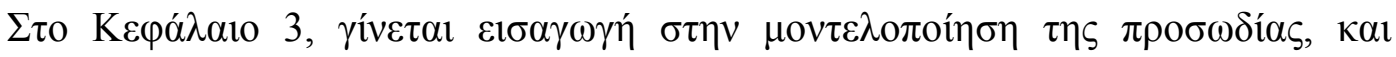

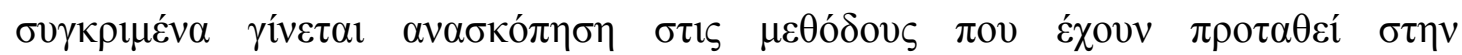

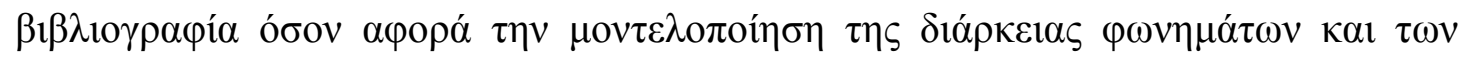

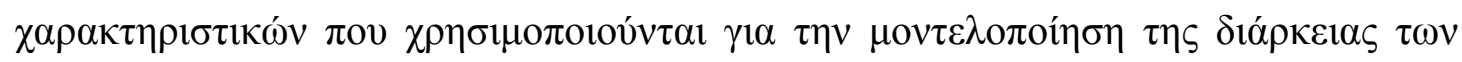

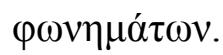

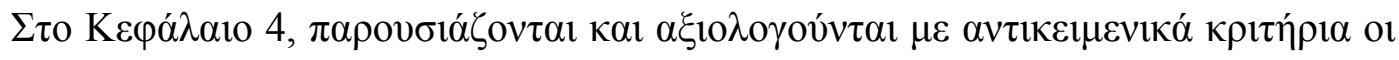

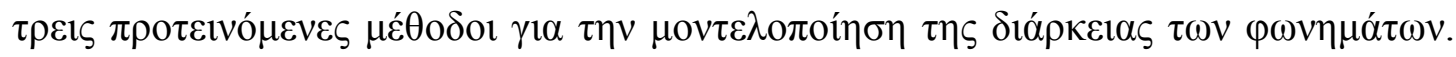

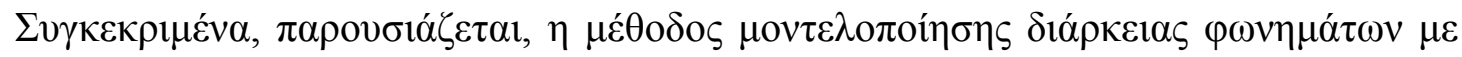

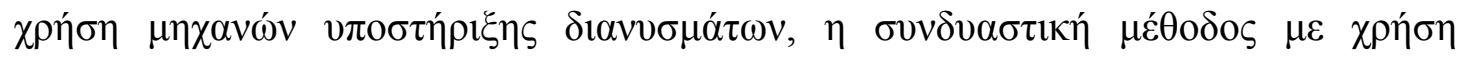

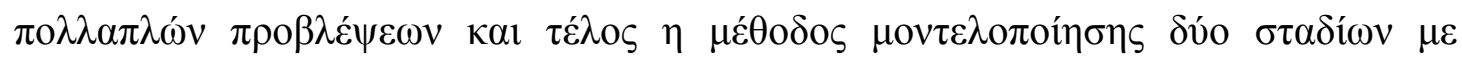

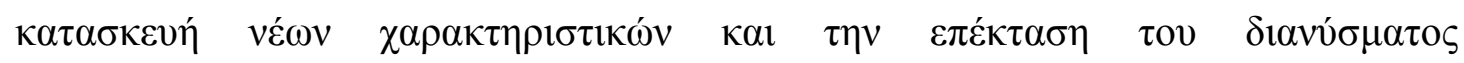

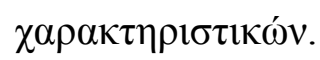

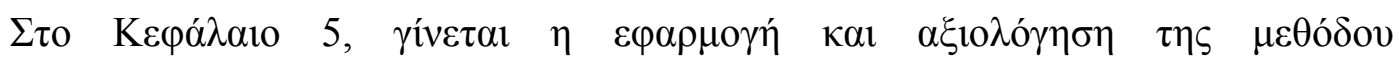

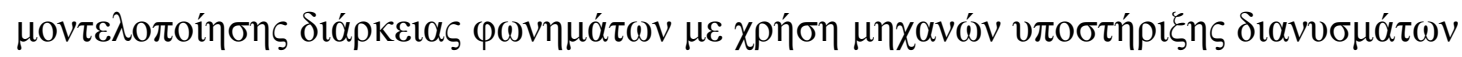

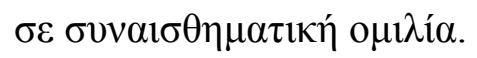

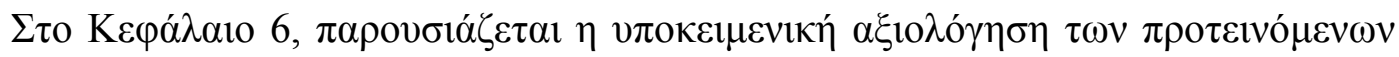

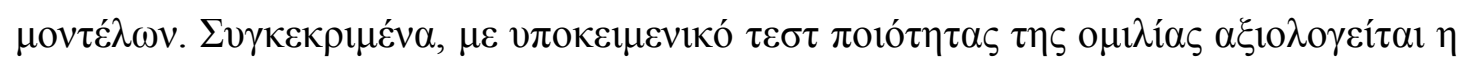

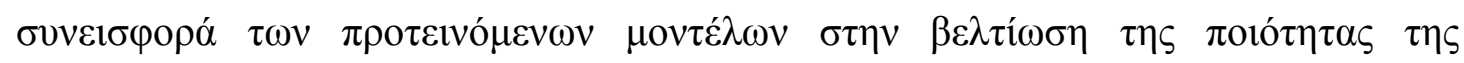

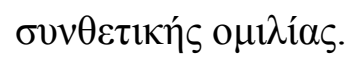

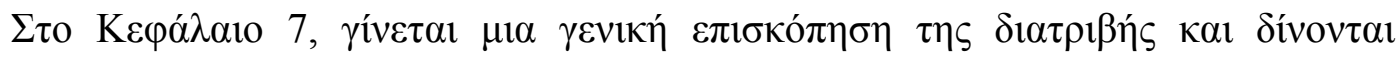

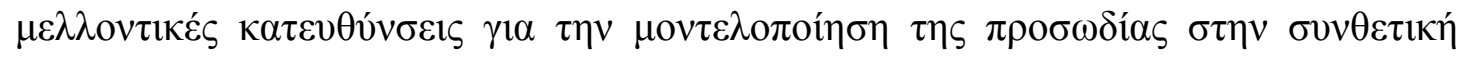

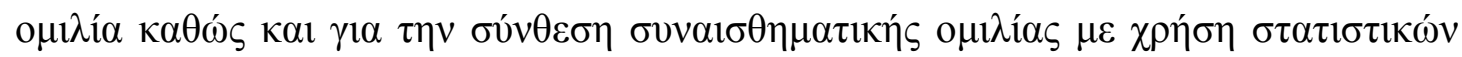

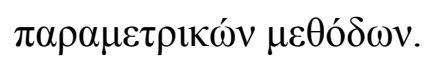

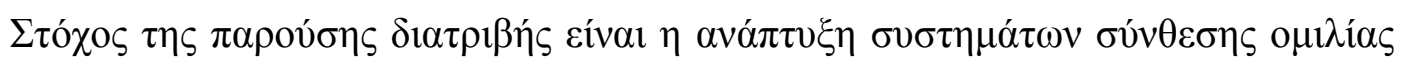

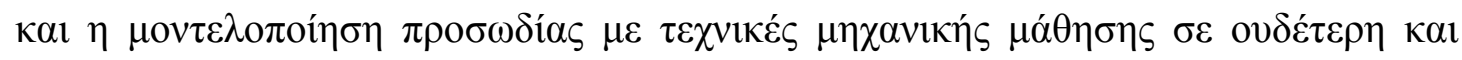




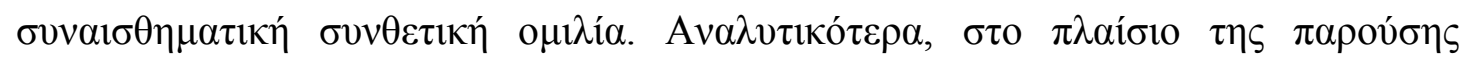

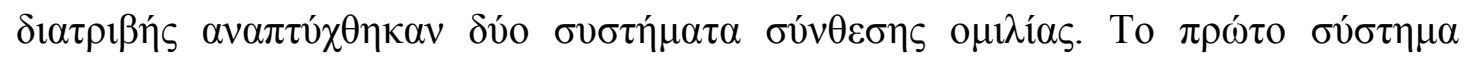

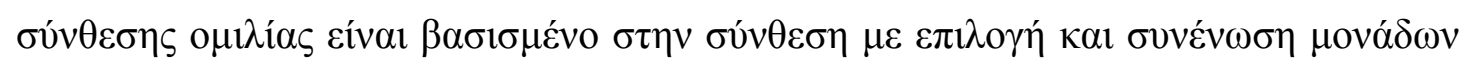

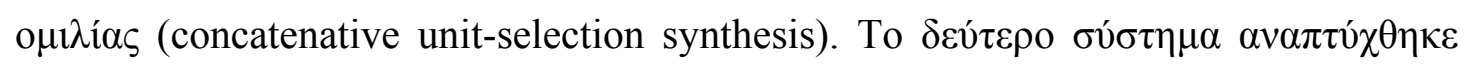

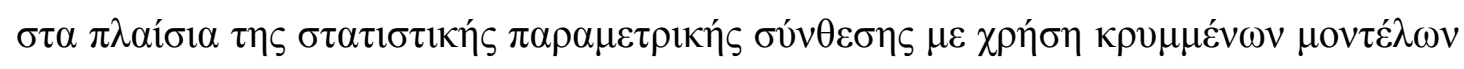

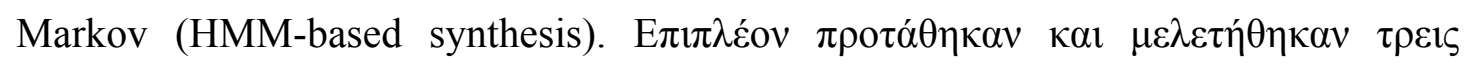

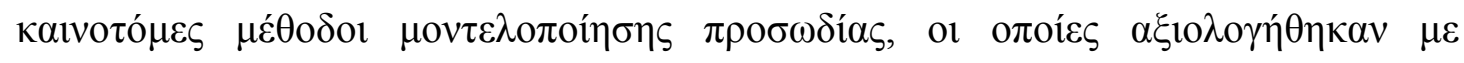

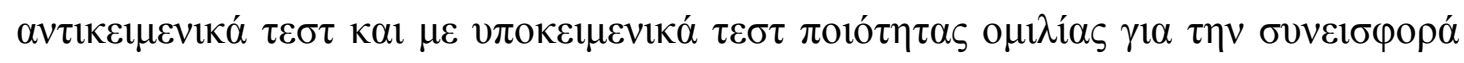

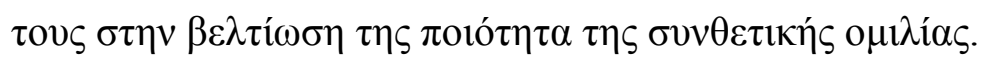

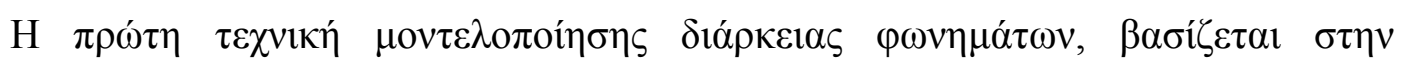

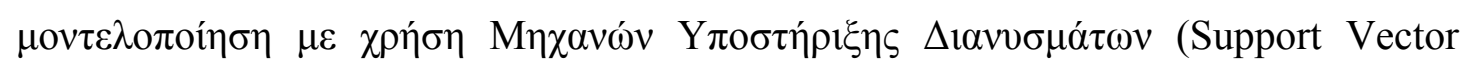

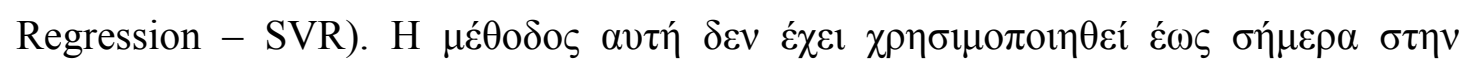

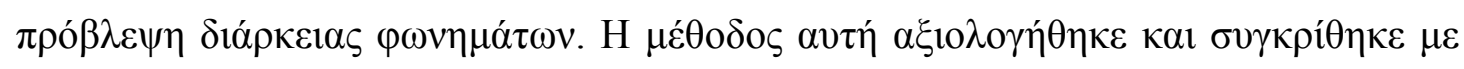

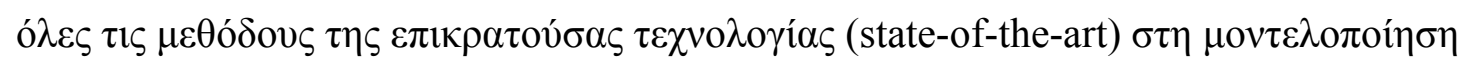

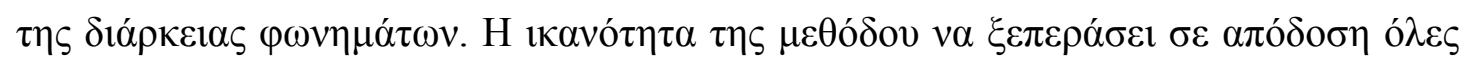

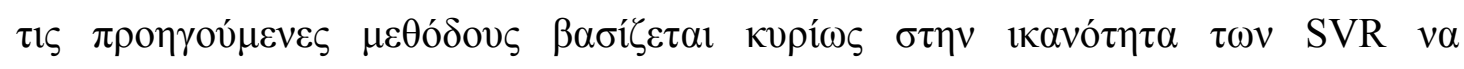

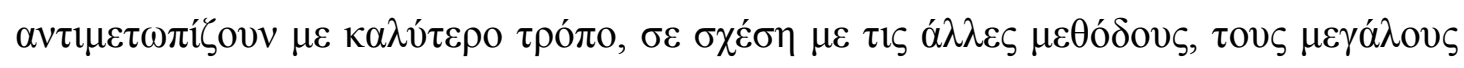

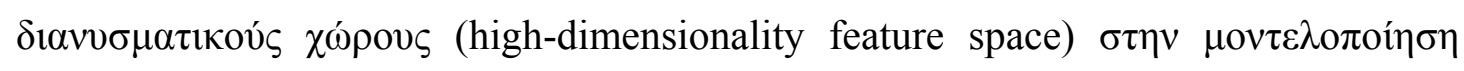

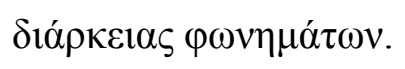

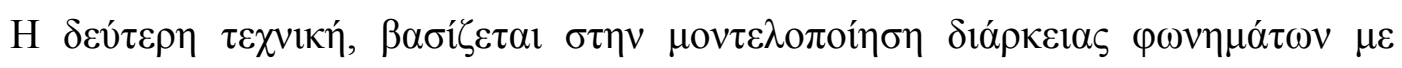

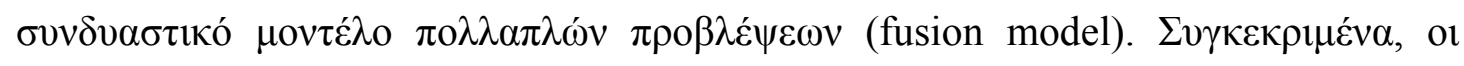

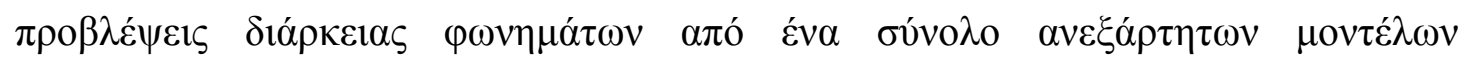

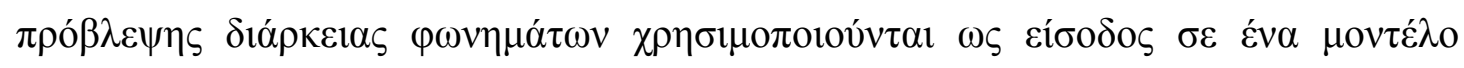

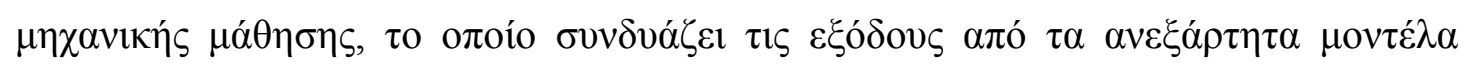

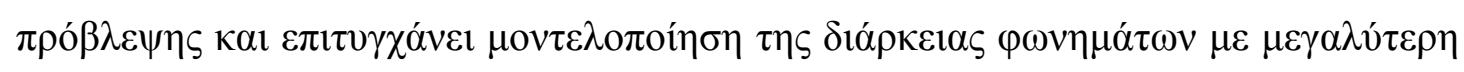

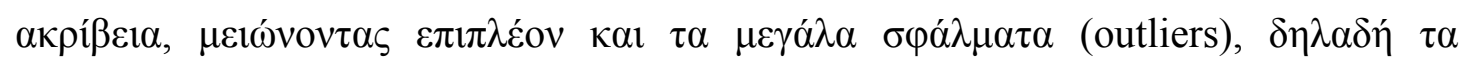

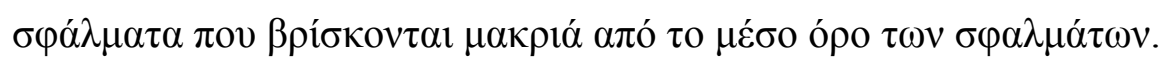

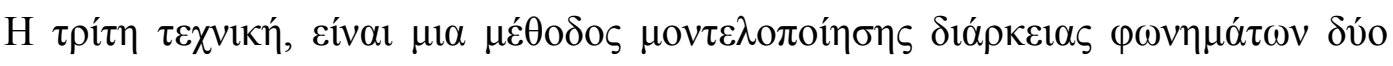

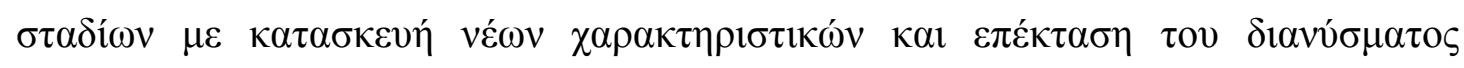

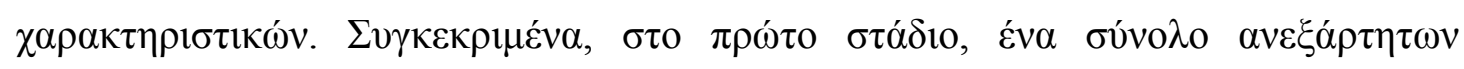

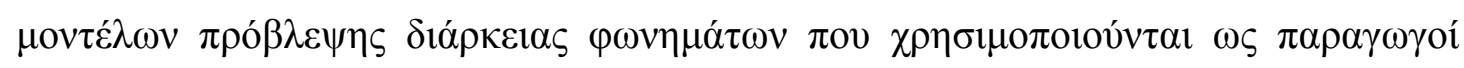

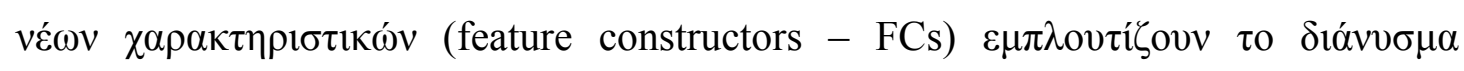

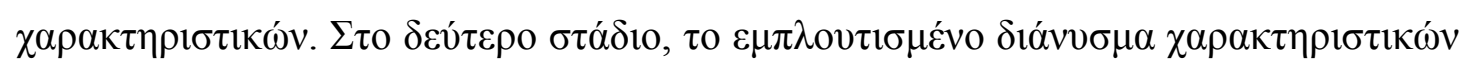

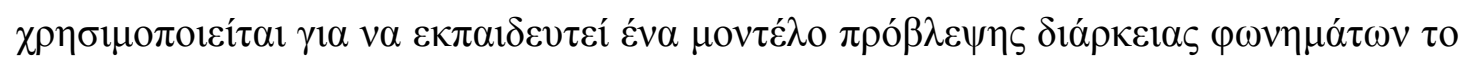




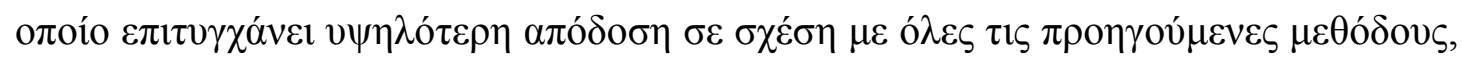

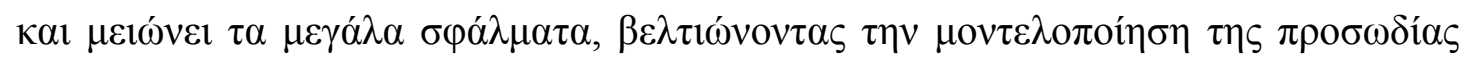

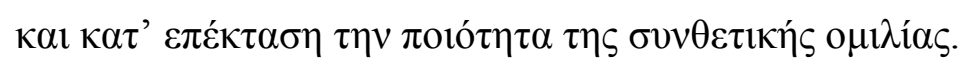

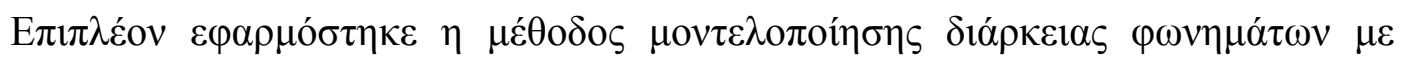

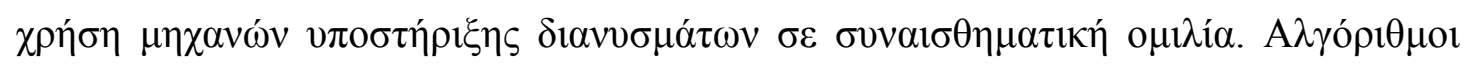

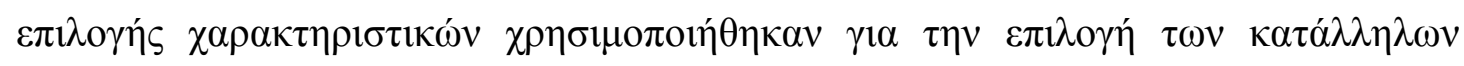

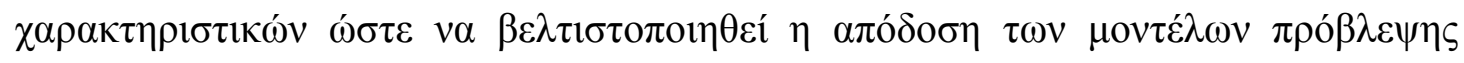

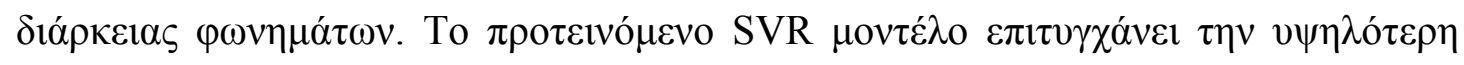

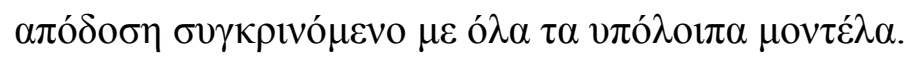

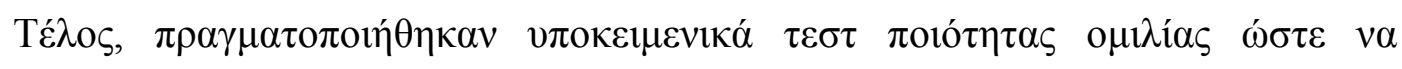

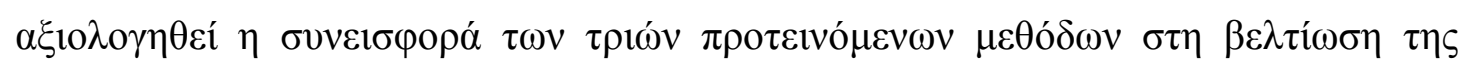

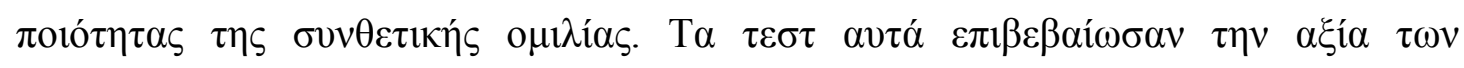

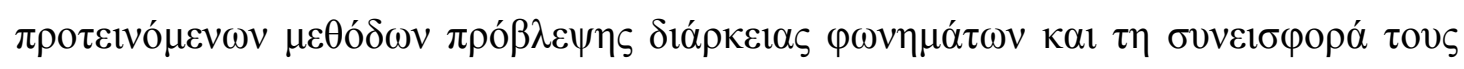

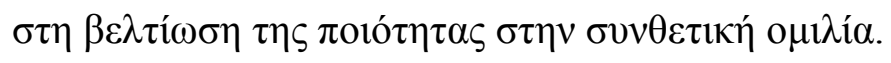




\section{CONTENTS}

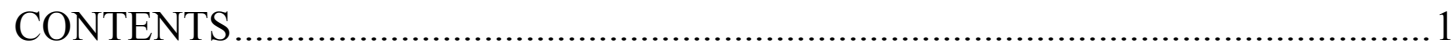

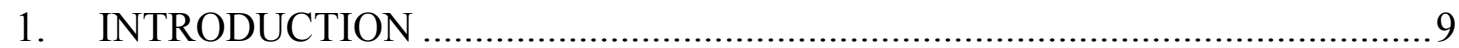

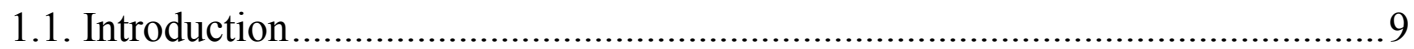

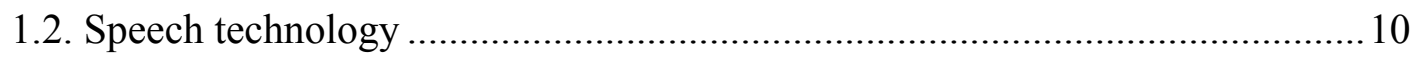

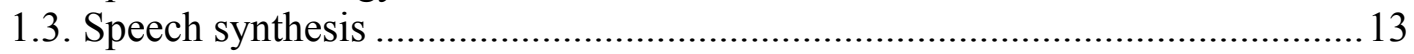

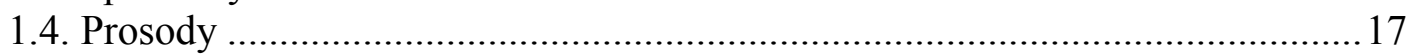

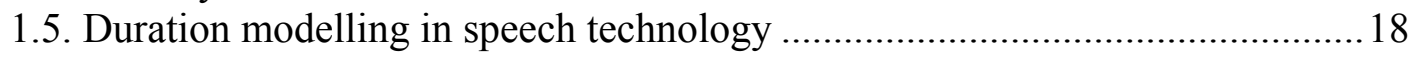

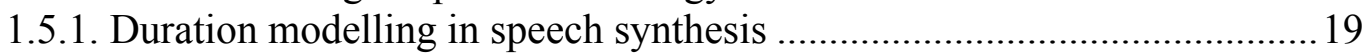

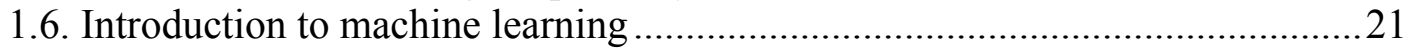

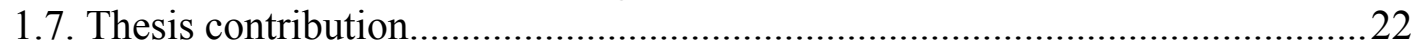

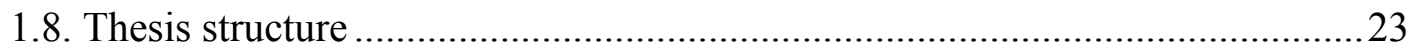

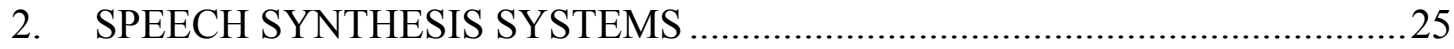

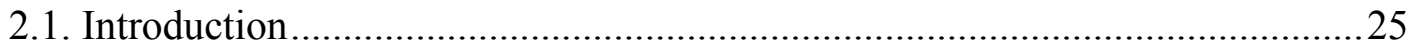

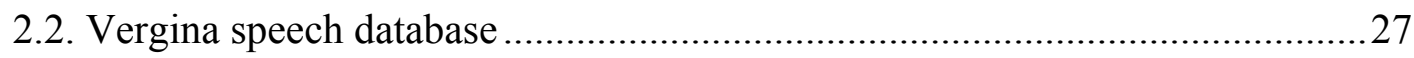

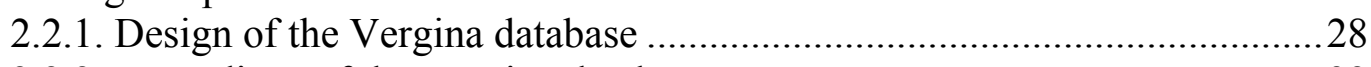

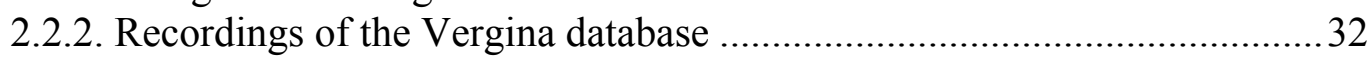

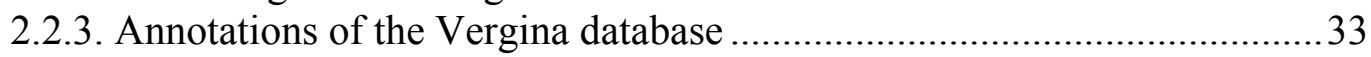

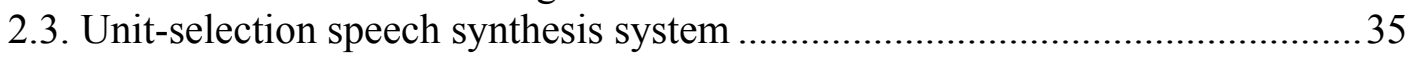

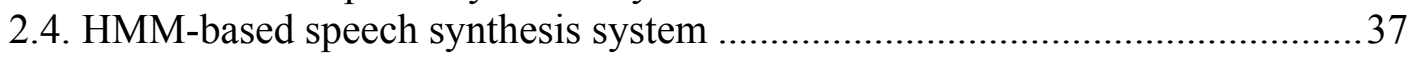

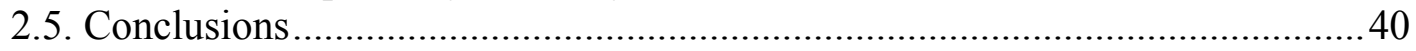

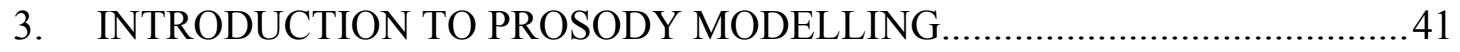

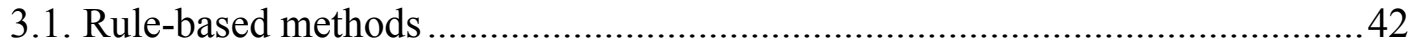

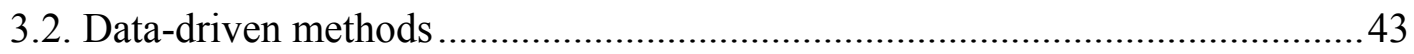

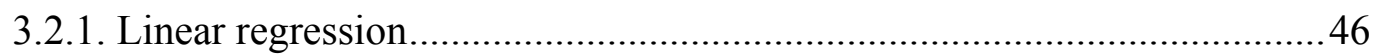

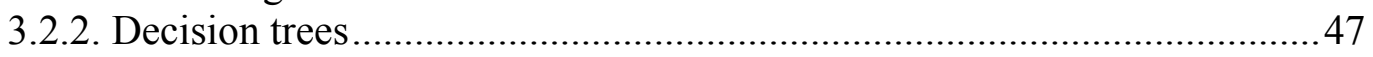

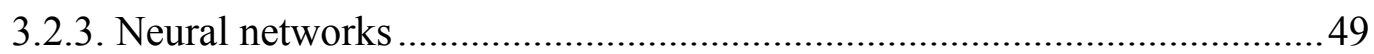

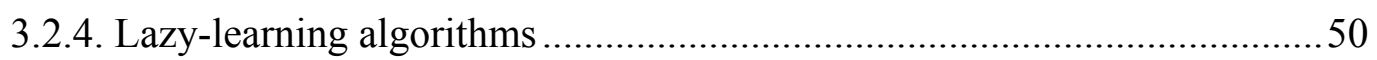

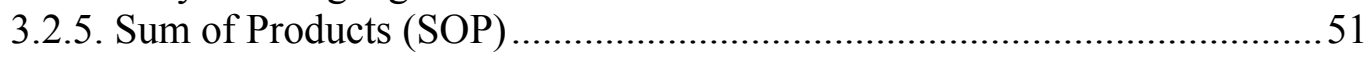

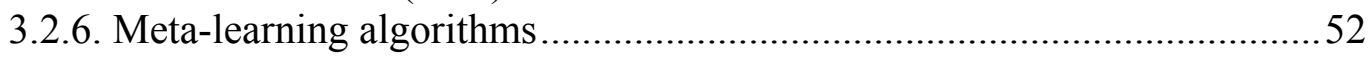

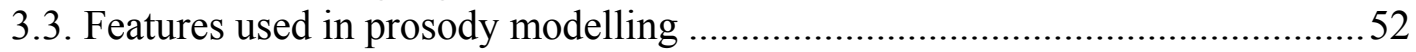

4. PROPOSED PROSODIC MODELS BASED ON MACHINE LEARNING

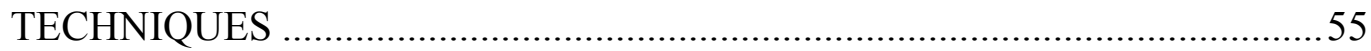

4.1. Prosody modelling with support vector regression......................................56

4.1.1. Introduction to support vector regression ...............................................57

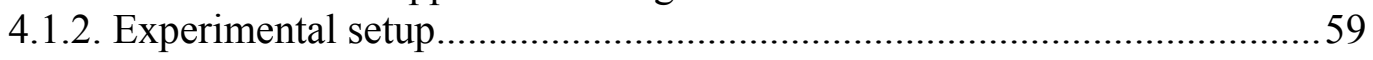

4.1.2.1. Phone duration models using other algorithms................................59

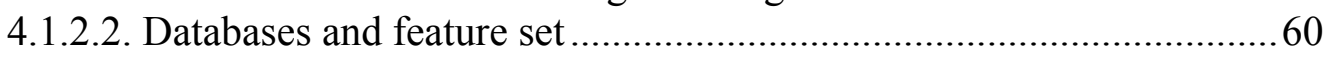

4.1.2.3. Performance evaluation metrics.....................................................6 63

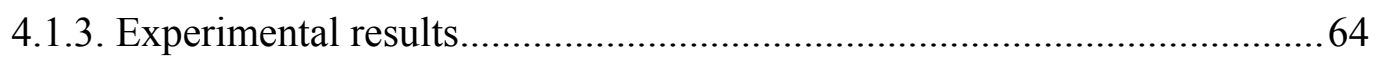

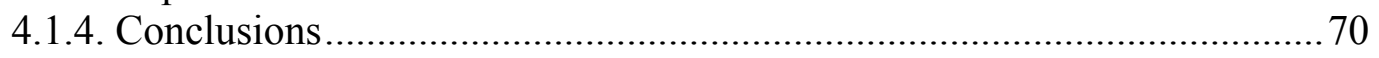

4.2. Fusion of multiple predictions using support vector regression ...................... 71

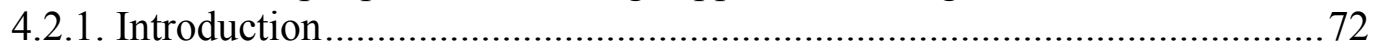

4.2.2. Fusion of models using support vector regression................................. 73

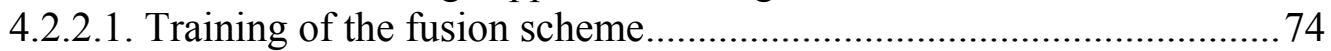

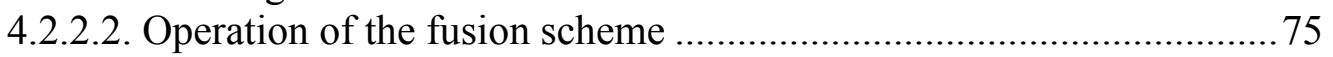

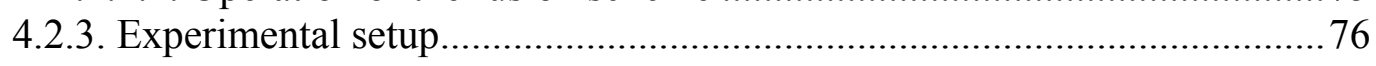

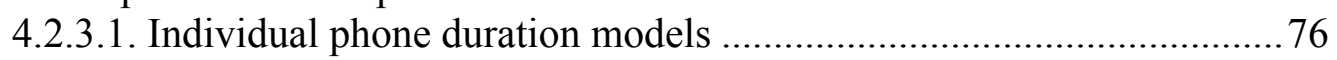

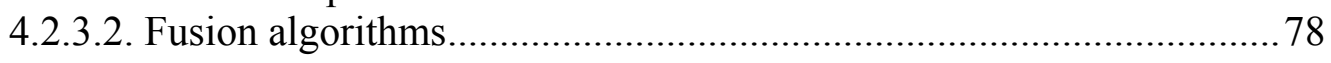




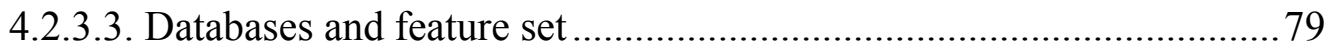

4.2.3.4. Performance evaluation metrics......................................................... 80

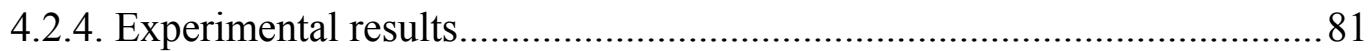

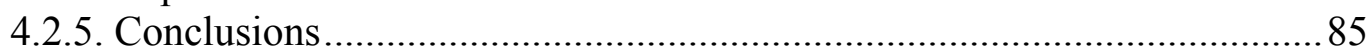

4.3. Two-stage prosody modelling with feature construction and feature vector

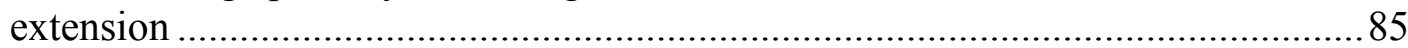

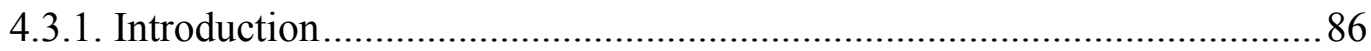

4.3.2. Two-stage scheme with feature construction and feature vector extension

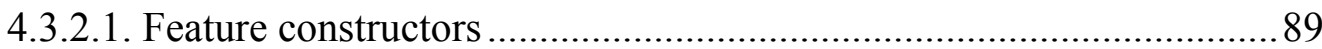

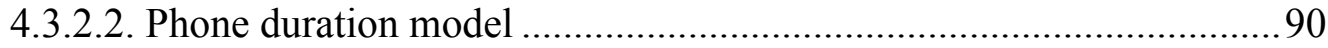

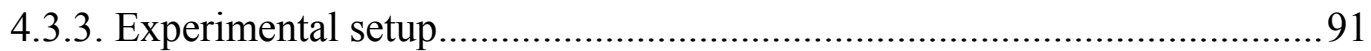

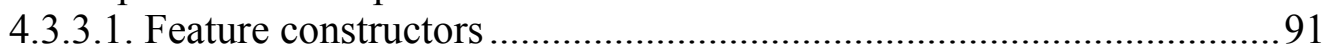

4.3.3.2. Phone duration model in the second stage ...................................... 92

4.3.3.3. Databases and feature set .............................................................. 93

4.3.3.4. Performance evaluation metrics....................................................... 94

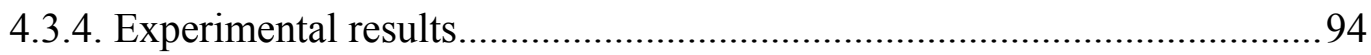

4.3.4.1. Additional experiments with feature ranking .................................97

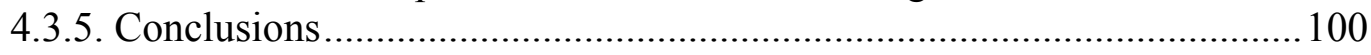

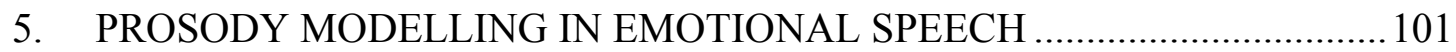

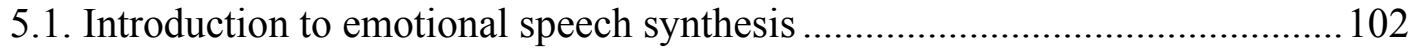

5.2. Introduction to prosody modelling in emotional speech................................ 105

5.3. Phone duration modelling in emotional speech ............................................ 106

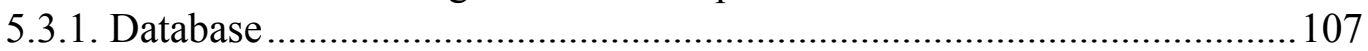

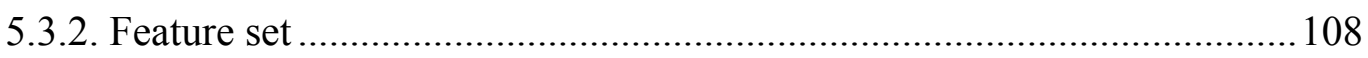

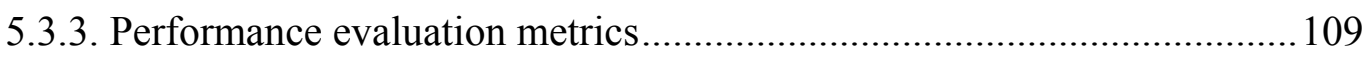

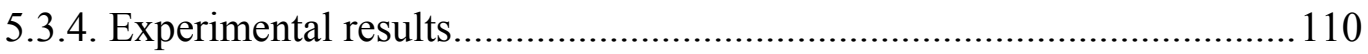

5.4. Implementation of feature selection algorithms ....................................... 112

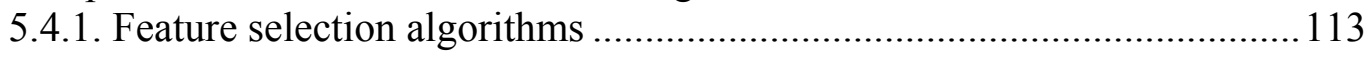

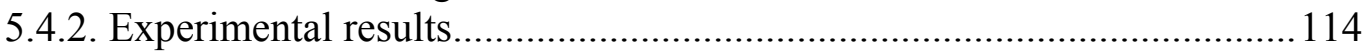

5.5. Support vector regression models in emotional speech ................................121

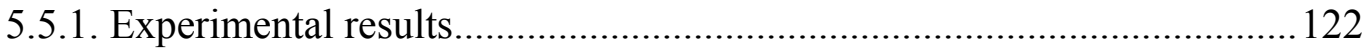

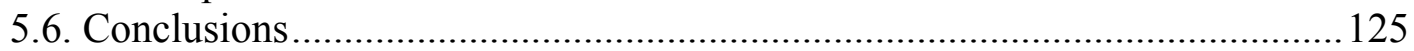

6. PERCEPTUAL EVALUATION OF THE PROSODIC MODELS ..................127

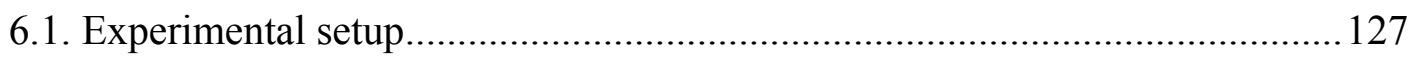

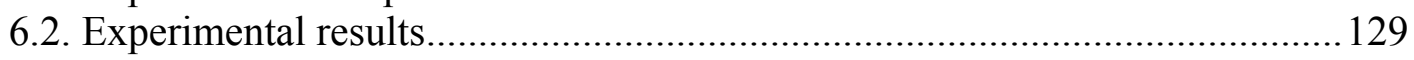

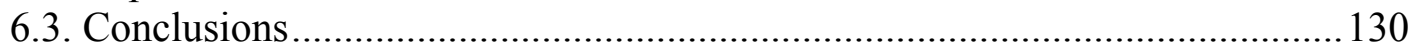

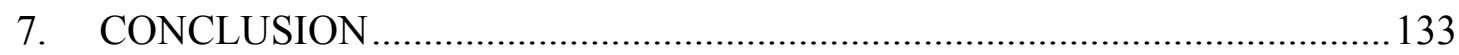

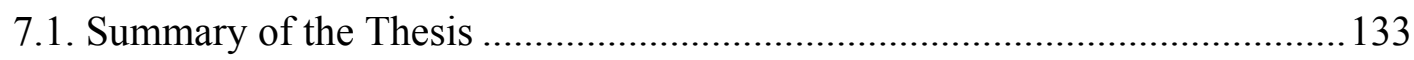

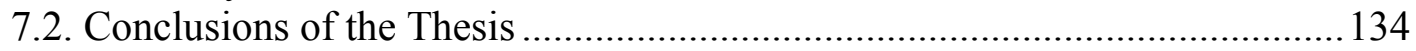

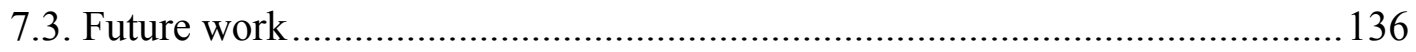

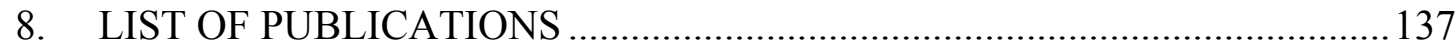

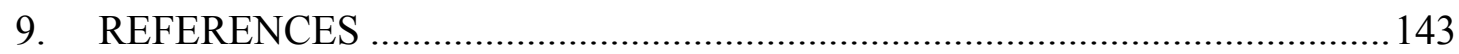

EXTENDED SUMMARY OF THE PHD DISSERTATION IN GREEK

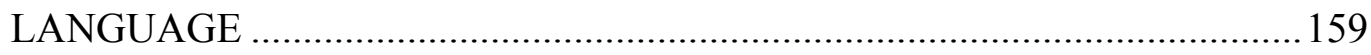




\section{List of Figures}

Fig. 1.1 The general diagram of the speech communication chain (Denes and Pinson, 1993)....................................................11

Fig. 1.2 The general block diagram of a dialog system.....................12

Fig. 1.3 Categories of speech technology...............................13

Fig. 1.4 General block diagram of a TTS system (van Santen et al., 1997)...14

Fig. 1.5 General block diagram of the frontend (Benesty et al., 2007).........14

Fig. 1.6 General block diagram of a phone duration model..................22

Fig. 2.1 The number of words per sentence............................... 30

Fig. 2.2 Diagram of the target and join cost functions........................36

Fig. 2.3 Viterbi search for the sequence of diphones of the word " $\varepsilon \alpha$ " (one).37

Fig. 2.4 Overview of a typical HMM-based speech synthesis system (Zen et al.,

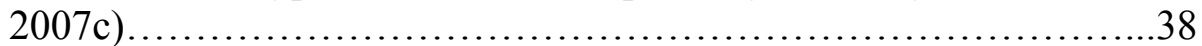

Fig. 2.5 Concatenation of context-dependent HMMs for generating the word "Éva" (one) ....................................................

Fig 4.1 Mean Absolute Error (MAE), standard deviation of absolute error (STD of AE) and Root Mean Square Error (RMSE) in milliseconds, for individual SMOreg and the second-best phone duration prediction algorithms on: (a) the KED TIMIT database, and (b) the WCL-1 database....................................................66

Fig 4.2 Block diagram of the proposed fusion scheme, which exploits multiple dissimilar phone duration predictors, operating on a common input...74

Fig 4.3 Mean Absolute Error (MAE), standard deviation of absolute error (STD of AE) and Root Mean Square Error (RMSE) in milliseconds, for the individual SMOreg, and the fusion SMOreg-Fusion models on: (a) the KED TIMIT database, and (b) the WCL-1 database............84

Fig 4.4 Phone duration prediction: (a) the classical approach, (b) two-stage approach involving intermediate feature vector extension step........87

Fig 4.5 Block diagram of the two-stage phone duration modelling scheme, which involves feature construction and feature vector extension......89

Fig 4.6 Mean Absolute Error (MAE), standard deviation of absolute error (STD of AE) and Root Mean Square Error (RMSE) in milliseconds, for individual SMOreg-FC, the SMOreg-FCF and the SMOreg-FVE models on: (a) the KED TIMIT database, and (b) the WCL-1 database......................................................96

Fig 5.1 Weighted average values of standard deviations in milliseconds of phone durations for all the emotions... 121 


\section{List of Tables}

Table 1.1. The three levels of prosody representation.......................17

Table 2.1. Characteristics, advantages and drawbacks of unit-selection and HMM-based speech synthesis approaches.......................27

Table 2.2. The twenty most frequently occurred words in the database along with their pronunciation............................................... 31

Table 2.3. The twenty most frequently occurred diphones in the database........31

Table 2.4. Vergina Speech Database phone set along with the respective symbols of the IPA alphabet.............................................32

Table 2.5. Mean duration, standard deviation and number of occurrences of the phones of Vergina speech database...............................35

Table 4.1 (a). Mean Absolute Error (MAE), standard deviation of absolute error (STD of AE) and Root Mean Square Error (RMSE) in milliseconds, for the eight individual phone duration prediction algorithms on the KED TIMIT database ...........................................64

Table 4.1 (b). Mean Absolute Error (MAE), standard deviation of absolute error (STD of AE) and Root Mean Square Error (RMSE) in milliseconds, for the eight individual phone duration prediction algorithms on the WCL-1 database................................................65

Table 4.2. Root Mean Square Error in milliseconds, per phonetic category for the eight individual phone duration prediction algorithms on: (a) the KED TIMIT database, and (b) the WCL-1 database........................67

Table 4.3 (a). Root Mean Square Error in milliseconds, per phone for the eight individual phone duration prediction algorithms on the KED TIMIT database.

Table 4.3 (b). Root Mean Square Error in milliseconds, per phone for the eight individual phone duration prediction algorithms on the WCL-1 database.....................................................70

Table 4.4. Mean Absolute Error (MAE), standard deviation of absolute error (STD of AE) and Root Mean Square Error (RMSE) in milliseconds, for the fusion scheme, implemented with the average linear combination and the best-case selection fusion algorithms on: (a) the KED TIMIT database, and (b) the WCL-1 database.................82

Table 4.5. Mean Absolute Error (MAE), standard deviation of absolute error (STD of AE) and Root Mean Square Error (RMSE) in milliseconds, for the various fusion techniques on: (a) the KED TIMIT database, and (b) the WCL-1 database.........................................83

Table 4.6. Mean Absolute Error (MAE), standard deviation of absolute error (STD of AE) and Root Mean Square Error (RMSE) in milliseconds, for the baseline FCF scheme and for the proposed FVE scheme on: (a) the KED TIMIT database, and (b) the WCL-1 database................95

Table 4.7. Mean Absolute Error (MAE), standard deviation of absolute error (STD of AE) and Root Mean Square Error (RMSE) in milliseconds, for the various feature subsets on the PDMs on: (a) the KED TIMIT database, and (b) the WCL-1 database...........................98 
Table 5.1. RMSE values in milliseconds for the different categories of emotional speech....

Table 5.2. MAE values in milliseconds for the different categories of emotional speech.

Table 5.3. CC values for the different categories of emotional speech..............111

Table 5.4. The number of features selected by the two feature selection algorithms, RReliefF and CFS, for the different categories of emotional speech. ...

Table 5.5. RMSE values in milliseconds for the different categories of emotional speech. Each phone duration model was evaluated for the full feature set, i.e. without feature selection (NoFS), and with feature selection based either on the RReliefF or the CFS methods.

Table 5.6. MAE values in milliseconds for the different categories of emotional speech. Each phone duration model was evaluated for the full feature set, i.e. without feature selection (NoFS), and with feature selection based either on the RReliefF or the CFS methods. .118

Table 5.7. CC values for the different categories of emotional speech. Each phone duration model was evaluated for the full feature set, i.e. without feature selection (NoFS), and with feature selection based either on the RReliefF or the CFS methods 119

Table 5.8. RMSE values in milliseconds for the different categories of emotional speech. For each emotion the best model with (WithFS) and without feature selection (NoFS) and the SVR performance with (RReliefF or CFS) and without (NoFS) feature selection... .123

Table 5.9. MAE values in milliseconds for the different categories of emotional speech. For each emotion the best model with (WithFS) and without feature selection (NoFS) and the SVR performance with (RReliefF or CFS) and without (NoFS) feature selection.

Table 5.10. $\mathrm{CC}$ values in milliseconds for the different categories of emotional speech. For each emotion the best model with (WithFS) and without feature selection (NoFS) and the SVR performance with (RReliefF or CFS) and without (NoFS) feature selection. 124

Table 6.1. Mean opinion score (MOS) scale for the perceptual evaluation tests for: (a) the KED TIMIT database, and (b) the WCL-1 database......128

Table 6.2 (a). Perceptual evaluation tests in MOS scale. MOS score and the standard deviation (STD) of MOS, and the MAE and the RMSE of the respective models for the KED TIMIT database

Table 6.2 (b). Perceptual evaluation tests in MOS scale. MOS score and the standard deviation (STD) of MOS, and the MAE and the RMSE of the respective models forthe WCL-1 database. 130

Table 6.3. Wilcoxon test on the results of the perceptual evaluation test for: (a) the KED TIMIT database, and (b) the WCL-1 database. 130 


\section{Abbreviations}

AI - Artificial Intelligence

ANN - Artificial neural network

$\mathrm{CC}$ - Correlation Coefficient

DSP - Digital Signal Processing

FC - Feature Constructors

FVE - Feature Vector Extension

HMM - Hidden Markov Model

LPC - Linear Predictive Coding

LR - Linear Regression

MAE - Mean Absolute Error

MFCC - Mel-frequency cepstral coefficient

MLSA - Mel Log Spectrum Approximation

MOS - Mean Opinion Score

PDM - Phone Duration Model

QMT1 - Quantification method type 1

QP - Quadratic Programming

RMSE - Root Mean Square Error

SMO - Sequential Minimal Optimization

STD - Standard Deviation

STD of AE - Standard Deviation of Absolute Error

SVM - Support Vector Machines

SVR - Support Vector Regression 


\section{Chapter 1}

\section{INTRODUCTION}

In this chapter, a brief introduction to speech processing and consequently to speech synthesis is made. Furthermore, the basic concept of prosody is presented along with a brief introduction to machine learning and how it is applied in phone duration modelling. Finally, the Thesis contribution and structure are presented.

\subsection{Introduction}

Speech constitutes the most natural and widespread way of communication among humans. The development of various fields of technology and science allowed the development of systems and tools which can be used for automating processes which are related to speech. As an example, the use of humans as operators in telephone centres has been replaced by automatic systems of service via telephone (call-centres). Specifically for the case of applications that function under stable conditions of noise or way of speaking, the modern speech technology allows the replacement of humans by dialog systems (Mporas, 2009).

Over the last decades, the development of systems concerning human computer interaction over speech has been increased considerably. Human computer interaction includes the transmission of messages from human to machine, meaning the transformation of natural speech into text (i.e. speech recognition), and the 
transmission of messages from machine to human, meaning the transformation of automatically produced text to synthetic speech (i.e. speech synthesis). The increasing rate in the development of such systems is mainly owed to the development of databases with recordings of speech of big volume (i.e. enough hours of speech) (Huang et al., 2001).

The development of large databases with recorded speech from different speakers and under different conditions and environments allowed the development of automatic speech recognition systems independent of the speaker, and big vocabulary, contrary to older systems that were usually limited to one speaker and to a limited vocabulary (e.g. recognition of digits, or individual words). Furthermore the development of large databases led to the replacement of the rule-based methods, widely used in various fields of speech technology, e.g. the formant and articulatory methods concerning speech synthesis, by data-driven (statistical modelling) such as hidden Markov model (HMM)-based synthesis and concatenative synthesis (corpusbased unit-selection, diphone).

\subsection{Speech technology}

Speech is the basic mean of communication among people and along with the written are the two main means of exchanging opinions, views, ideas, knowledge and culture. Nonetheless, the oral communication is faster, more direct and more convenient than written, making oral speech mandatory in all the fields of human life and communication.

The development of technology over the last decades, allowed the creation of systems, mainly focused on specific tasks, which are in position of replacing humans in specific tasks. It is obvious that such systems, which are in direct communication and contact to humans, in order to be friendly to the user, must follow the way of communication among humans. Such systems may be automatic call-centres, services of information (tourist information, cinema), financial services (bank), security control for access to rooms or buildings with speech, smart houses and cars, etc. Speech technology is the field of science that deals with the communication between human and machine (human-computer interaction - HCI) with the most natural way, 
which is speech. Moreover, speech technology is interested in the fusion of speech with other types of communication, such as visual communication, gestures, etc (Huang et al., 2001).

In Fig. 1.1 the "speech chain", which is the transmission of information (meaning-concept) from the speaker to the listener, is shown. Initially certain semantics (concepts) are produced by the brain. These concepts are converted into sentences of natural speech by the brain and through the neurons of the nervous system, a command is given to all the muscles involved in the speech production mechanism to produce the corresponding air wave through the vocal tract producing speech. Specifically, the lung's muscles create an air pressure that passes through the vocal cords, the glottis. Depending on whether, at that moment, voiced or unvoiced sounds are meant to be produced, the vocal cords are found respectively to be vibrating or relaxing, creating an air flow with impulse or turbulent form respectively. Consequently, the air flow coming out of the glottis, passes through the pharynx and the larynx, and afterwards through the mouth cavity, in combination or not with the nasal cavity, and comes out with the form of speech signal. Depending on what sound must be produced at that moment, the vocal tract with the jaw and tongue take the suitable place of articulation. On the listener's side, the ear receives the speech signal and the ear's sensors convert it to the corresponding neural signals. Consequently, the nerves transmit this information to the brain, which in turn converts it to concepts.

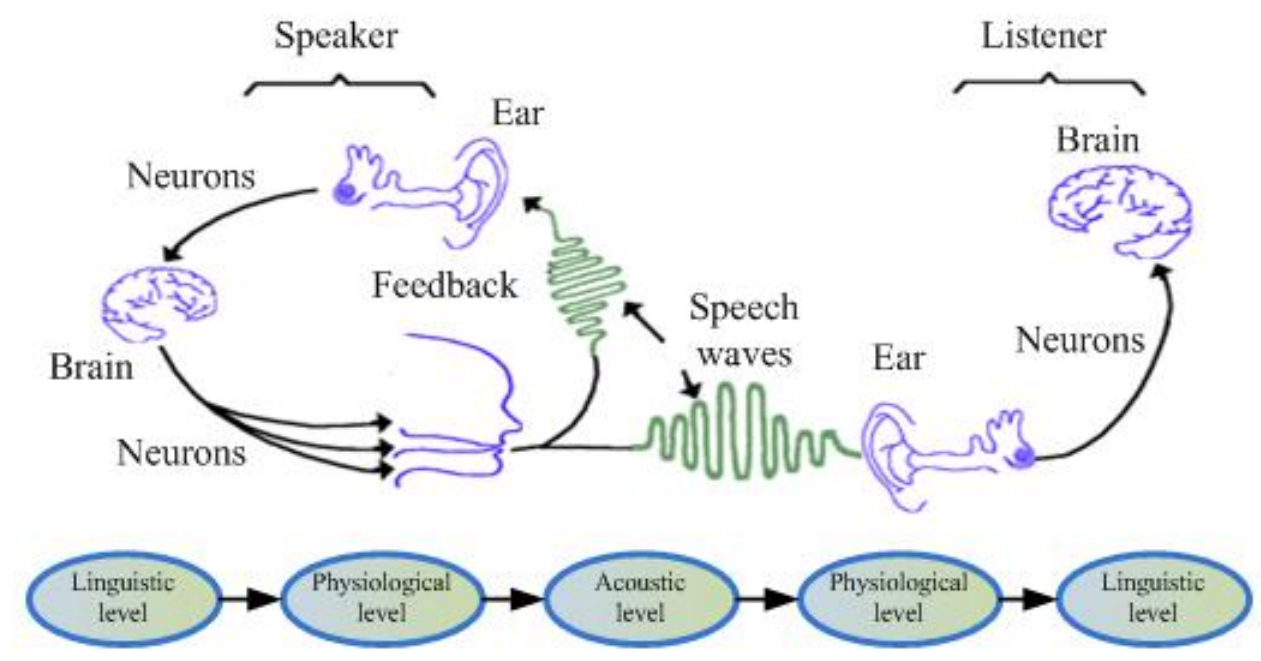

Fig. 1.1. The general diagram of the speech communication chain (Denes and Pinson, 1993). 
Speech is actually a group of basic-fundamental sounds. These sounds are the phonemes and are different for each language. Each phoneme has no contextual meaning by each own, however combinations of these sounds and in particular, in specific order, correspond to meanings, i.e. words of each language. Of course the meaning that corresponds to each word can be differentiated for the same sequence of phonemes, according to where the word is stressed, or according to the words that precede or follow. Even though each language has its own group of phonemes, all the spoken languages are considered subsets of a global set of phonemes which is called International Phonetic Alphabet (IPA) (IPA, 1999).

Speech technology tries to follow or even improve the processes that take place during the oral communication between two people. The communication process of human-computer (HCI) is presented in Fig. 1.2, and represents the basic structure of a general dialog system.

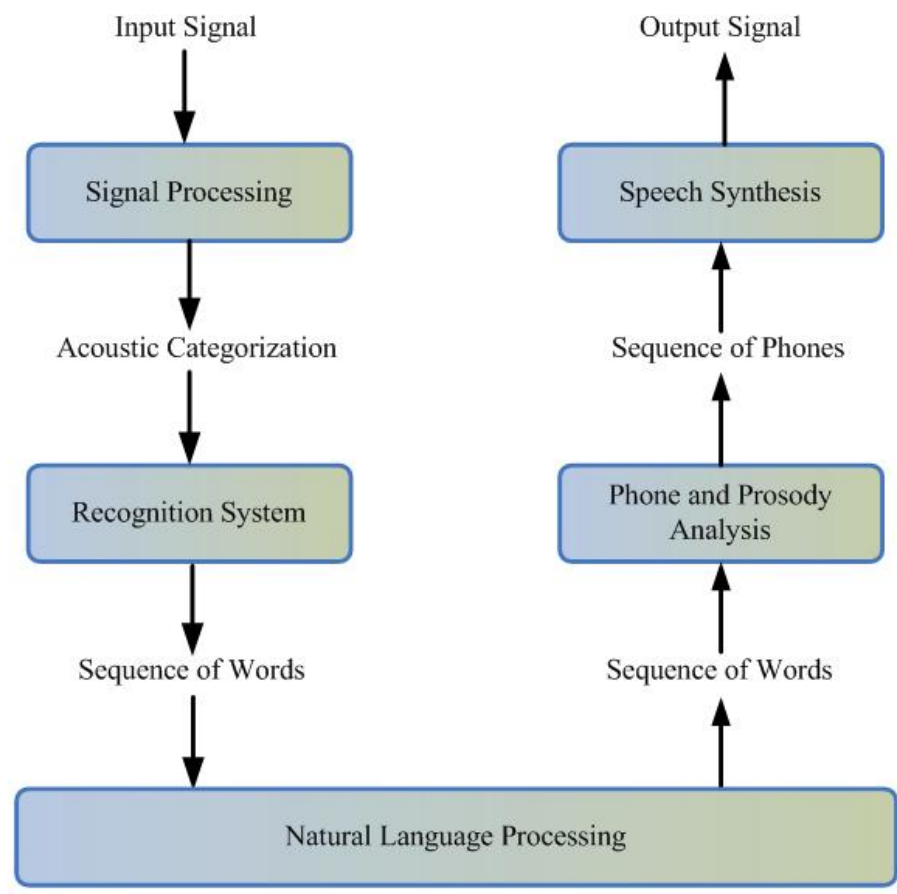

Fig. 1.2. The general block diagram of a dialog system.

As it is shown in Fig. 1.2, the speech signal from the human-user is processed and led to an automatic speech recognition (Automatic Speech Recognizer - ASR), where it is converted to a sequence of recognized words. Consequently, a natural language processing system, which is the heart of the dialog system, will process the input data. This system deals with the conversion of text to concepts, natural language 
understanding (NLU) process, the processing of concepts and the production of new ones, which is the response of system to the human-user, the conversion of these new concepts into natural speech with a natural language generation (NLG) system, and finally, the conversion of text that corresponds to the response of system to the human-user through synthetic speech, with a text-to-speech conversion (TTS) system.

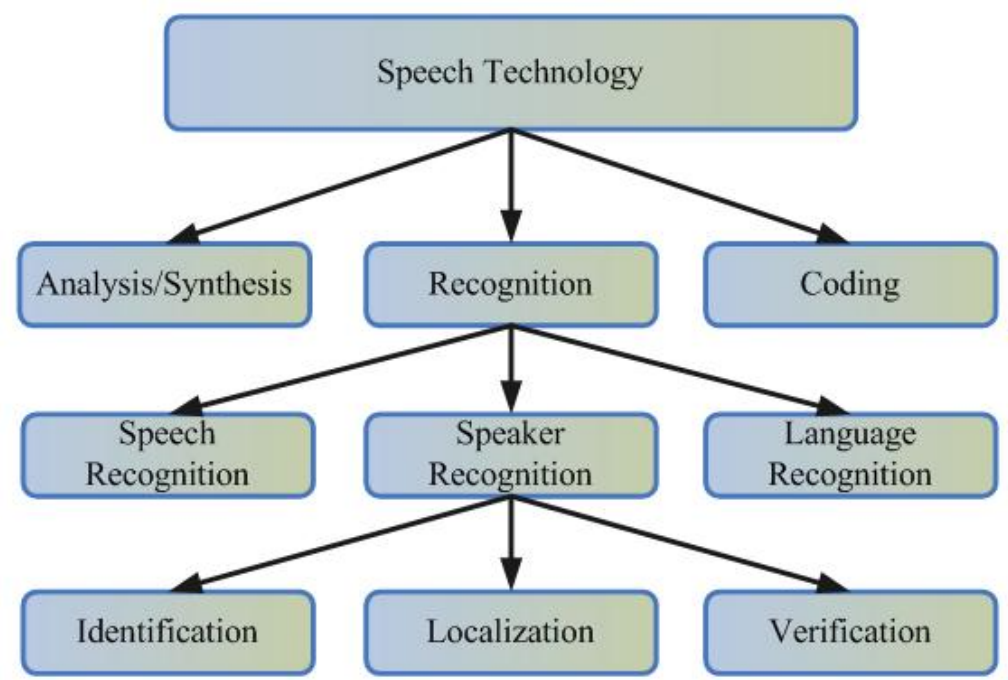

Fig. 1.3. Categories of speech technology.

In this point, it should be mention that speech technology includes a wide variety of systems and sub-systems responsible for the complete human-computer interaction. All these systems can be categorized in various fields as it is shown in Fig. 1.3.

\subsection{Speech synthesis}

Speech synthesis is the artificial production of human-like speech. Speech synthesizers are also called text-to-speech (TTS) systems since their task is to convert normal (or tagged) text to speech (Allen et al., 1987). Over the years, speech synthesis has been used in a great range of applications covering different fields of human life and human needs such as helping people with visual impairment as screen readers, or people with dyslexia or other reading difficulties as a learning tool, or in the entertainment area in games and animation producing various voices and speaking styles. A general block diagram of a TTS system is shown in Fig. 1.4. The frontend of 
the TTS takes text as input and produces control information as output. The frontend is basically a text analysis component. The backend uses the control information and produces synthetic speech at the output.

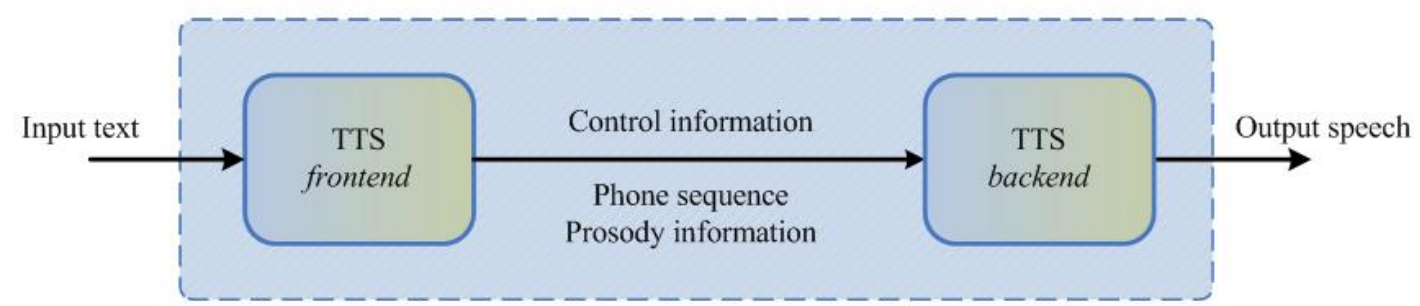

Fig. 1.4. General block diagram of a TTS system (van Santen et al., 1997).

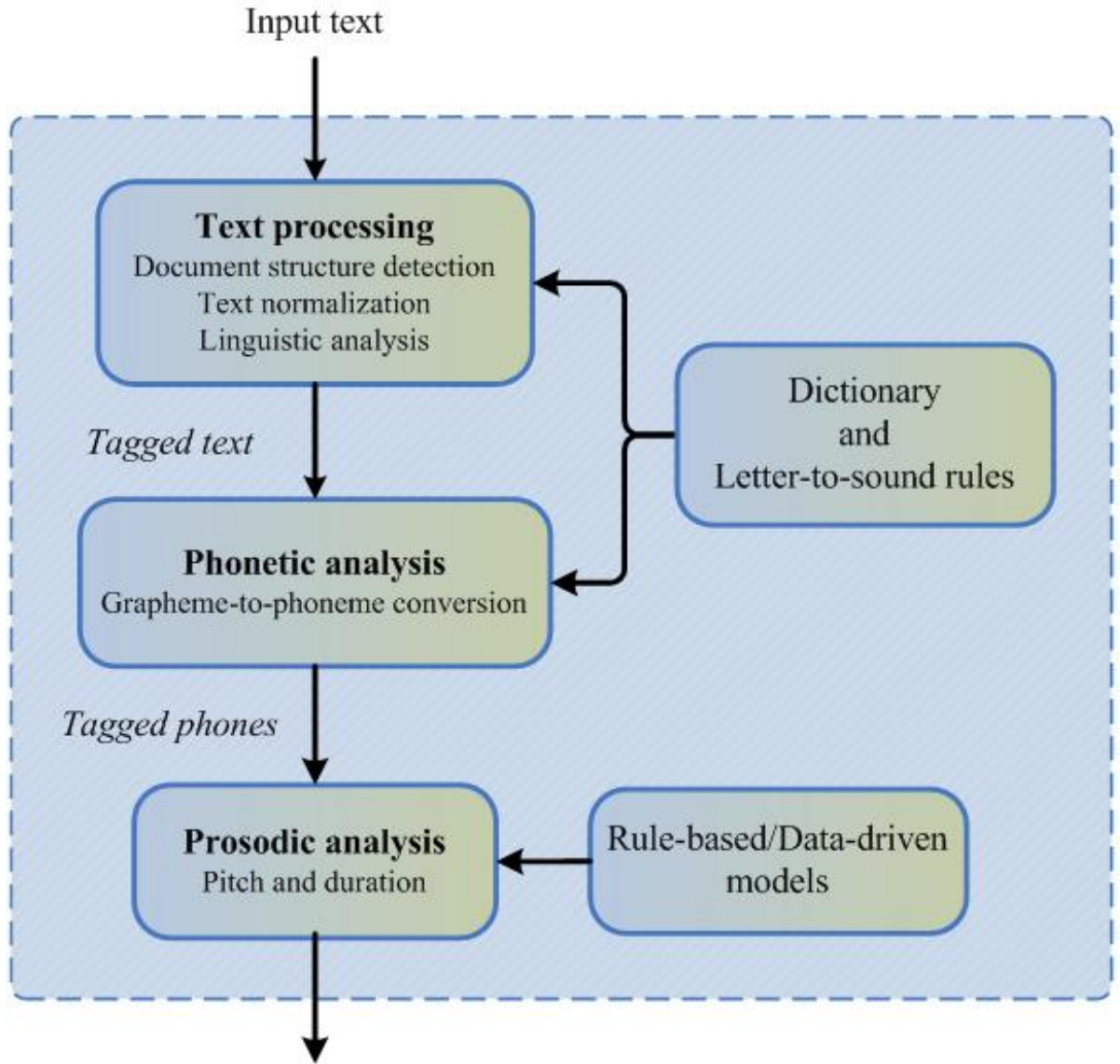

Control information

Sequence of phones, pitch, duration

Fig. 1.5. General block diagram of the frontend (Benesty et al., 2007).

The main tasks of the frontend of a TTS are presented in Fig 1.5 (Benesty et al., 2007). In brief, the frontend provides document structure detection (e.g. recognizing email headers or addresses) and text normalization (e.g. dealing with abbreviations or 
acronyms). Moreover, frontend performs linguistic analysis (e.g. morphological analysis for proper pronunciation or dealing with ambiguities in text) on the input text. Furthermore, the phonetic analysis component handles the phonetic representation of the words (e.g. pronunciation, grapheme-to-phoneme conversion), tagging the phones with information regarding the way they should be converted to sound (e.g. speaking style). Finally the prosody analysis component mainly determines the variations in time of the pitch and duration over the sequence of the phones.

All the aforementioned information produced in the frontend of the TTS is consequently passed to the TTS backend where synthetic speech is produced using a specific synthesis method. A basic categorization of the synthesis methods is to rulebased (e.g. articulatory and formant synthesis) and corpus-based (e.g. concatenative unit-selection, hidden Markov model synthesis). These methods are briefly described next.

The articulatory synthesis is based on the modelling of the vocal tract and the human speech production mechanism. This method is mainly used as a research tool for better understanding of the human speech production mechanism rather than as a system for commercial applications. On the other hand, formant synthesis instead of modelling the vocal tract, tries to model only the input/output characteristics using formant filters. Formant synthesis following the theory of the source-filter model of speech production, which is based on the independence of the source (lungs and vocal cords) and the filter (vocal tract), models the formant frequencies and bandwidths.

The corpus-based methods are mainly three, the concatenation with fixed inventory, the unit-selection and the HMM-based synthesis. The first, the concatenation with fixed inventory, usually referred to as diphone synthesis, was the first concatenative technique introduced in the speech synthesis field. This method is based on the use of one relatively small database containing one instance for each diphone - a unit of speech starting from the middle of one phone till the middle of the next phone - of the language. Since one instance is used for each diphone, the recording of the diphones is done with as neutral and flat prosody as possible. During synthesis, the appropriate prosody (mainly pitch and duration) is applied on the diphones using digital signal processing methods such as linear predictive coding (LPC). The quality of the synthetic speech is quite lower than the one of unit-selection 
synthesis, which is described next, but needs much less resources and has lower computational cost.

On the other hand, the unit-selection corpus-based synthesis is based on the use of large databases (many hours of speech) containing hundreds or thousands instances of each unit (usually phones or diphones). This method is the most widely and commonly used speech synthesis method over the last years. Unit-selection synthesis produces synthetic speech with the highest quality in contrast to all the other speech synthesis methods. The reasoning behind this is that no or almost no modification of the speech units is done since the units are selected based on some criteria, from the database and concatenated together producing the synthetic speech. Two cost measures are used for selecting the units, the join cost function depending on the spectral similarity at the unit boundaries in order to avoid discontinuation effects and the target cost function depending on the matching of the prosody of the units to the prosodic information produced by the frontend of the system.

Finally, HMM-based synthesis is a statistical parametric corpus-based method. In HMM-based synthesis, speech parameters such as the spectrum, fundamental frequency and phone duration are modelled using $n$-states (usually $n$ is equal to 3 ) HMMs. In synthesis phase a sequence of HMMs is used to generate these speech parameters based on maximum likelihood criterion and produce synthetic speech through a Mel Log Spectrum Approximation (MLSA) filter. This technique, in contrast to the unit-selection corpus-based approach, even though it produces synthetic speech of lower quality, offers the advantage of modelling different speaking styles and emotions with the use of limited databases. This is achieved by applying a model adaptation algorithm, such as the Maximum Likelihood Linear Regression (MLLR) algorithm (Tamura et al., 2006; Yamagishi et al. 2004) or MAPbased (Maximum A Posteriori) modification (Gauvain and Lee, 1994; Nakano et al., 2006; Ogata et al., 2006), using a small amount of speech uttered by the target speaker. The target speaker is not restricted only to different speakers with different voice characteristics but also can be characterized by different speaking styles or even different emotions. 


\subsection{Prosody}

Prosody can be regarded as the implicit channel of information in the speech signal that conveys linguistic, paralinguistic and extra-linguistic information related to communicative functions. Such functions are the linguistic functions of prominence (stress and accent), the phrasing, the discourse segmentation, the information about expression of emphasis, attitude, assumptions, the emotional state of the speaker, the information about the identify of the speaker (particular with respect to habitual factors). These functions provide to the listener clues supporting the recovery of the verbal message (Clark and Yallop, 1995; Laver, 1980; Laver, 1994). The accurate modelling and control of prosody in a text-to-speech system leads to synthetic speech of higher quality. Prosody is shaped by the relative level of the fundamental frequency, the intensity and last but not least by the duration of the pronounced phones (Dutoit, 1997; Furui, 2000).

Table 1.1. The three levels of prosody representation.

\begin{tabular}{ccc}
\hline Acoustic & Perceptual & Linguistic \\
\hline Fundamental frequency (F0) & Pitch & Tone, intonation, aspect of stress \\
\hline Amplitude, energy, intensity & Loudness & Aspect of stress \\
\hline Duration & Length & Aspect of stress \\
\hline
\end{tabular}

Prosody can be represented in three different but none the less correlated levels, the acoustic, the perceptual and the linguistic levels (see Table 1.1) (Dutoit, 1997). The first, the acoustic representation (fundamental frequency, amplitude and duration) can be measured directly by using hardware (devises) or software (algorithms). The second, the perceptual level (pitch, loudness and length) is correlated with the prosodic events as perceived by the listener. In this level, the aspects of prosody cannot be measured objectively but only subjectively through the listener's perspective. Finally the third level, the linguistic level, corresponds to the symbolic representation of prosody in an utterance. This level represents a structural interpretation of prosodic data defined by linguists (Dutoit, 1997).

Duration and pitch (fundamental frequency - f0) are not completely independent. Many of the factors of the semantic level that determine duration also influence the determination of pitch in speech (Huang et al., 2001). Even though this dependency 
exists, for practical reasons these two aspects of prosody are usually treated separately (van Santen, 1994). Pitch generation in synthetic speech is a procedure divided in two parts. First, the symbolic prosody (phrase breaks, accents, tones, etc.) (Huang et al., 2001) is determined, in order to represent all the factors, i.e. pragmatic, syntactic and semantic features, that affect pitch and consequently, the generation of the pitch contours based on the symbolic representation is done. On the other hand, the duration controls the rhythm and the tempo of speech and determines the speaking style of the speech (Yamagishi et al., 2008). Consequently, the flattening of prosody in a speech waveform would result in a monotonous, neutral, toneless and without rhythm synthetic speech, sounding unnatural, unpleasant to the listener or sometimes even scarcely intelligible (Chen et al., 2003). Along with pitch and duration but with a little less important role in prosody, amplitude, which is perceived as loudness by the listener, mainly helps in emphasizing or not specific parts of the speech helping the listener to focus on different parts of the it and meanings of phrase which is said.

\subsection{Duration modelling in speech technology}

Several areas of speech technology, among which automatic speech recognition (ASR), automatic segmentation (AS), speaker recognition (SR) and last but not least, speech synthesis (TTS), benefit from duration modelling. In hidden Markov model (HMM)-based ASR, implementing state duration models or explicit duration models either by using hidden semi-Markov models (HSMM) or other explicit duration modelling techniques, improve the speech recognition performance (Anastasakos et al., 1995; Bourlard et al., 1996; Jennequin and Gauvian, 2007; Levinson, 1986; Mitchell et al., 1995; Pols et al., 1996; Pylkkonen and Kurimo, 2004). For example, in (Anastasakos et al., 1995), instead of improving the durational behaviour at the state level, a method for modelling the segmental duration outside the HMM framework was proposed, improving the word error rate (WER) in respect to the baseline HMM by $7.9 \%$ in terms of relative improvement. Furthermore, in (Pylkkonen and Kurimo, 2004), a post-processor duration model (Juang et al., 1985), outperformed the baseline HMM by a relative improvement of $5.2 \%$ and $5.5 \%$ in letter error rate (LER) and word error rate (WER), respectively. Moreover, concerning the task of automatic 
segmentation, in ( $\mathrm{Wu}$ et al., 2005), an explicit duration modelling technique was proposed, implementing Gaussian distribution (Russell and Moore, 1985) as the probability distribution of the duration model and using a two-step-based approach. In this study, the main contribution of this model was to the reduction of the larger errors, i.e. larger than 30 milliseconds, achieving a relative reduction of $27 \%$ and $47 \%$ on a Chinese and a Japanese databases, respectively. Finally, significant improvement of the performance in the speaker recognition task was achieved by Ferrer et al. (2003), when duration-based speech parameters were used for the characterization of the speaker's voice. In this work, duration features at the level of word, phone or state, based on automatic speech recognition, when combined with speaker recognition system achieved a reduction in the identification error by $50 \%$. Finally, in TTS systems, the correct segmental duration contributes to the naturalness of synthetic speech (Chen et al., 1998; Klatt, 1976), as presented in the next subsection.

\subsubsection{Duration modelling in speech synthesis}

The prediction of the segments' durations is a task essential in speech synthesis no matter which method of synthesis is used. From the first mechanical TTS systems (Wolfgang von Kempelen "Talking Machine" 1791, UK Speaking Clock 1936, Homer Dudley 'VODER' 1939, Haskins Labs "Pattern Playback" 1950, Gunnar Fant's OVE speech synthesizer) till the rule-based, i.e. articulatory and formant synthesis methods, and the corpus-based, i.e. diphone (with fixed inventory), unitselection and HMM-based synthesis, the phone duration modelling was an integral task of the speech synthesizers implemented in each time in a different way and based on different approaches and technologies. Specifically in rule-based synthesis, phone duration modelling was implemented in TTS systems mainly through the use of rules determining the duration of each phone. The rule-based methods use manually produced rules, extracted from experimental studies on large sets of utterances, or based on previous knowledge. The extraction of these rules requires labour of expert phoneticians. The main disadvantage of the rule-based approaches is the difficulty to represent and tune manually all the linguistic factors, such as the phonetic, the morphological and the syntactic ones, which influence the segmental duration in speech. As a result, it is very difficult to collect all the appropriate (or even enough) 
rules without long-term devotion to this task (Klatt, 1987). Consequently the rulebased duration models are restricted to controlled experiments, where only a limited number of contextual factors are involved in order to be able to deduce the interaction among these factors and extract the corresponding rules (Rao and Yegnanarayana, 2007).

Later on, in concatenative speech synthesis techniques, such as in diphone synthesis, phone duration modelling implemented through machine learning techniques (data-driven phone duration modelling) was used in order to modify through DSP approaches - each diphone and give the appropriate prosody in synthetic speech. Nowadays, in unit-selection corpus-based synthesis which is also a concatenative method, no or almost no modification of the speech units is done during the concatenation of them (Hunt and Black, 1996). In this approach, phone duration models play a very important role not by modifying the units but in the selection of the appropriate units (along with other factors such as the f0 or the context of each unit in the word and phrase) through the target cost function (Section 1.3 - Speech synthesis). In (Teixeira, 2004), a study on artificial neural networks (ANNs)-based phone duration models was reported. A standard ANN model was compared to an alternative model using one ANN model for each type of phone showing that a $5.7 \%$ relative improvement in terms of root mean square error (RMSE), led to a relative improvement in the quality of speech by $4 \%$ in terms of mean opinion score (MOS). In (Krstulovic et al., 2008), it was reported that modelling phone duration with a Quantification method type 1 (QMT1) model (Hayashi, 1952), instead of HMMs, in a unit-selection corpus-based TTS, contributed to the improvement of synthetic speech in terms of quality with a relative improvement in MOS scale of $3.7 \%$ for "command" sentences and 7.4\% for "Wh-questions" sentences. These results are very important since, this kind of sentences, are rich in prosodic phenomena making even more important the accurate modelling of prosody.

Moreover, in HMM-based synthesis, duration modelling can be applied to the synthesis procedure in two ways, internally and externally. In the former, internally by the HMMs, through the state transition probabilities (Silen et al., 2010; Yoshimura et al., 1998; Zen et al., 2007a) or modifications of them (Latoree et al., 2010; Gao et al., 2008), using HSMMs (Zen et al., 2004) or other explicit duration modelling techniques, the duration of the phones is determined. In the latter, the phones' durations are determined externally, through an external data-driven (machine 
learning) phone duration model (Latoree et al., 2010). In this study, a QMT1 model (Hayashi, 1952), was used modelling externally the phone duration instead of determining duration explicitly with HSMM. Perceptual results showed a preference of the listeners for the QMT1 model over the explicit HSMM model by $12 \%$.

Finally, it should be pointed out that phone duration model and prosody in generally are essential components in emotional synthesis (Eide et al., 2004; Jiang et al., 2005; Strom et al., 2006). Since prosody is much more intense and rich in emotional speech, the accurate control of it plays a very important role in emotional speech synthesis.

\subsection{Introduction to machine learning}

Machine learning is the field of science which deals with the design and development of algorithms and techniques for creating and evolving or recognizing patterns and structures using empirical data, improving their future performance. A more precise definitions is given next (Mitchell, 1997):

A computer program is said to learn from experience $E$ with respect to some class of tasks $T$ and performance measure $P$, if its performance at tasks in $T$, as measured by $P$, improves with experience $E$.

Machine learning is highly correlated with Artificial Intelligent (AI) tasks such as: recognition, classification and regression, clustering, decision making, prediction, etc. Two are the major learning types of machine learning algorithms, the supervised and the unsupervised. In the former, the supervised learning, the output values or the ground truth (reference) values in respect to specific data are known and used by the machine learning algorithm for its training. These algorithms usually belong to the category of classification and regression techniques and their main goal is to create a mapping function of the input data to the output values. The later, the supervised learning, is mainly correlated to clustering problems where the output values are unknown. In these algorithms, the main goal is to analyse and organize the data according to their characteristics.

Prosody modelling and specially phone duration modelling is a regression problem. The data $W$ consists of pairs $\left(X_{j}, y_{j}\right)$ where $X_{j}$ are the features concerning 
$j$ th instance and $y_{j}$ is the output value (i.e. phone duration) of the $j$ th instance. In the training phase, the goal is to find a mapping function $f$ that closely correlates the features to the output values. Consequently, this function is used in the operational phase, where unknown data $U$ containing only features without the output values are fed to the model predicting the phones' durations.

\section{Training phase}
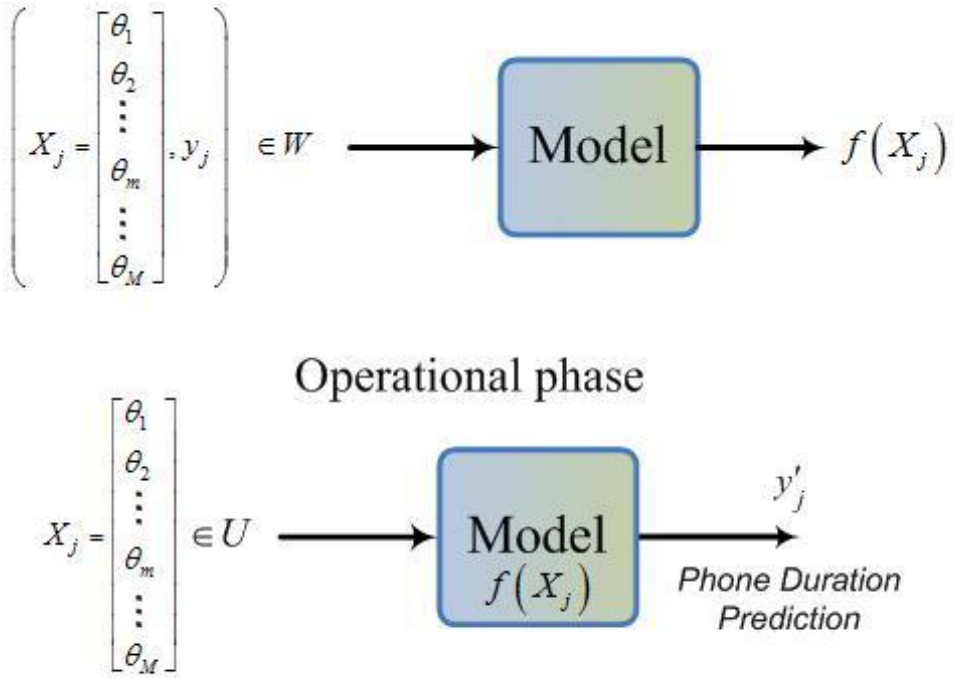

Fig. 1.6. General block diagram of a phone duration model.

\subsection{Thesis contribution}

In this Thesis, three machine learning techniques for prosody modelling are presented and evaluated. Specifically:

- The first proposed technique introduced in this Thesis is the Support Vector Regression (SVR) approach. SVR has never been used before in the phone duration modelling task. The SVR are compared with the state-of-the-art models in phone duration modelling. Their ability to outperform all the other models is mainly based on their advantage of coping in a better way with highdimensionality feature spaces and small amount of training data in respect to the other models used in phone duration modelling. 
- The second proposed phone duration modelling scheme is a fusion scheme based on the combination of independent phone duration models operating in parallel. Specifically, the predictions from a group of dissimilar and independent to each other individual phone duration models are fed to a machine learning algorithm, which reconciles and fuses the outputs of the individual models, yielding more precise phone duration predictions and furthermore reducing the outliers.

- The third proposed scheme is a two-stage phone duration modelling approach which builds on a number of independent feature constructors (FCs) employed in the first stage, and a phone duration model (PDM), which operates on an extended feature vector in the second stage. The extended feature vector is obtained by appending the phone duration predictions estimated by the FCs to the initial feature vector. The two-stage phone duration model manages to reduce the phone duration prediction error and furthermore reduces the outliers.

Furthermore a study concerning phone duration modelling in the filed of emotional speech is presented in this Thesis. Feature selection techniques are applied in order to evaluate the features and select the most appropriate ones improving the phone duration modelling accuracy.

Finally, apart from the objective evaluation of the proposed phone duration modelling schemes, subjective evaluation was performed in order to measure the impact of the proposed schemes to the quality of the synthetic speech and more precise to the naturalness of it.

\subsection{Thesis structure}

The structure of this Thesis is described in the following.

In Chapter 2, the design and development of a speech database for the Greek language is presented along with the development of two speech synthesis systems, a unit-selection corpus-based synthesis system and an HMM-based statistical parametric speech synthesis system, developed during this dissertation. 
In Chapter 3, an introduction to prosody modelling is presented. Specifically, an overview is done concerning the phone duration modelling approaches and techniques which have been studied in the literature over the years. Furthermore, a description of the features which have been used in this task is done.

In Chapter 4, the three proposed approaches for phone duration modelling are presented. Specifically, the support vector regression (SVR) model, the fusion scheme based on the combination of the predictions of individual phone duration models and the two-stage phone duration modelling scheme with feature construction and feature vector extension are described in detail and evaluated.

In Chapter 5, phone duration modelling is studied in emotional speech. Feature selection techniques are applied in order to evaluate the features and select the most appropriate ones improving the phone duration modelling accuracy. Furthermore, the first proposed approach, SVR, is applied and evaluated in emotional speech.

In Chapter 6, perceptual evaluation tests are presented in order to evaluate the improvement of the proposed phone duration modelling schemes in terms of improvement of the naturalness of synthetic speech.

In Chapter 7, a summary of the Thesis is given along with the conclusions and the feature work concerning phone duration modelling in neutral and emotional speech. 


\section{Chapter 2}

\section{SPEECH SYNTHESIS SYSTEMS}

In this chapter, the design and development of Vergina speech database, developed in support of research and development of corpus-based unit-selection and statistical parametric speech synthesis systems for Modern Greek language is presented. Moreover, two speech synthesis systems, a unit-selection corpus-based and an HMM-based statistical parametric speech synthesis system, developed during this dissertation, are presented.

\subsection{Introduction}

In Text-to-Speech (TTS) synthesis there are two major issues concerning the quality of the synthetic speech, namely the intelligibility and the naturalness (Dutoit, 1997; Klatt, 1987). The former refers to the capability of a synthesized word or phrase to be comprehended by the average listener. The latter represents how close to the human natural speech, the synthetic speech is perceived.

Over the last years the most widely used approach for high quality speech synthesis is the unit-selection corpus-based technique (Black and Taylor, 1997; Campbell and Black, 1996; Hunt and Black, 1996; Mobius, 2000). This approach is mainly based on runtime selection of the appropriate units of speech from a database and the concatenation of them with no or almost no speech processing of the selected 
speech units apart from the part where the concatenation takes place (Hunt and Black, 1996). Unit-selection synthesis exploits many hours of speech containing hundreds or thousands of instances for each unit (e.g., phones or diphones) and produces the highest quality synthetic speech among all the existing methods. This can be attributed to the fact that the selected from the database speech units are not modified at all or they are slightly modified. Even though the quality of the synthetic speech produced by unit-selection is very high, there is still no flexibility in the manipulation of the synthetic voice characteristics (e.g., speaking style, speech tempo and rhythm, emotions). The reasoning behind this drawback is that the characteristics of the synthetic speech follow the ones of the speech stored in the database since synthesis is carried out by concatenating speech waveforms selected from the database, making mandatory the creation of multiple databases, one for each speaker, separate speaking style or emotion to be synthesized, which is a very time consuming and expensive process.

In parallel with unit-selection corpus-based speech synthesis, the statistical parametric speech synthesis techniques have been developed with the hidden Markov model (HMM)-based approach to be the most commonly used one (Black, 2006; Ling et al., 2006; Yoshimura et al., 1999; Zen et al., 2007b). In contrast to unit selection speech synthesis, where actual instances of speech taken from a database are concatenated together to synthesize speech, in statistical parametric speech synthesis, the synthetic speech is produced by the proper manipulation of the parameters of a model offering the advantage of controlling the procedure and adapting the approach to different voices-speakers, languages or applications (Black et al., 2007). The main characteristics, advantages and drawbacks of unit-selection and HMM-based speech synthesis approaches are presented in Table 2.1. 
Table 2.1. Characteristics, advantages and drawbacks of unit-selection and HMM-based speech synthesis approaches.

\begin{tabular}{|c|c|}
\hline Unit-selection speech synthesis & HMM-based speech synthesis \\
\hline \multicolumn{2}{|c|}{ Characteristics } \\
\hline $\begin{array}{c}\text { Multi-instances of waveforms } \\
\text { Single tree for waveforms }\end{array}$ & $\begin{array}{c}\text { Statistics } \rightarrow \text { small footprint } \\
\text { Multiple tree for spectrum, duration and F0 }\end{array}$ \\
\hline $\begin{array}{l}\text { Advantage: } \\
\qquad \quad \text { Waveform concatenation } \\
\quad \rightarrow \text { High quality speech }\end{array}$ & $\begin{array}{l}\text { Disadvantage: } \\
\quad \quad \text { Vocoder-based synthesis } \\
\quad \rightarrow \text { buzzy sound of synthetic speech }\end{array}$ \\
\hline $\begin{array}{l}\text { Disadvantage: } \\
\text { - } \quad \text { Discontinuity } \\
\text { - } \quad \text { Hit or miss on selecting the units }\end{array}$ & $\begin{array}{l}\text { Advantage: } \\
\text { - Smooth synthetic speech no } \\
\text { discontinuity } \\
\text { - Stable } \\
\end{array}$ \\
\hline $\begin{array}{c}\text { Fixed voices following the characteristics of } \\
\text { the speech database }\end{array}$ & $\begin{array}{l}\text { Various voices tuning the voice } \\
\text { characteristics, adapting them. }\end{array}$ \\
\hline
\end{tabular}

In order to produce high quality synthetic speech, TTS methods rely on databases of high quality recordings, i.e. databases of clean and controlled speech are needed. The recordings have to be noise free (studio quality) and free of artifacts introduced by the speaker, such as breathe sounds, sounds of the lips, etc. Furthermore, the contents of the speech database must be phonetically rich and balanced with controlled prosody, and with utterances targeting at the domain for which the TTS is designed for. Consequently, the availability of large speech databases is a prerequisite for the unit-selection (Hunt and Black, 1996; Iwahashi et al., 1993) and the HMMbased speech synthesis (Tokuda et al., 2000; Yoshimura et al., 1999) approaches. Since Modern Greek is not a widely-spoken language, to this end only limited efforts have been invested in the development of corpus-based speech synthesis as well as the development of speech synthesis resources and tools (Fotinea and Tambouratzis, 2005; Tsiakoulis et al., 2008; Zervas et al., 2008).

\subsection{Vergina speech database}

In this subsection, the Vergina speech database, which was developed in support of research and development of unit-selection corpus-based and statistical parametric speech synthesis systems for Modern Greek language is presented. In the following, we describe the design, development and implementation of the recording campaign, as well as the annotation of the database. Specifically, a text corpus of approximately 
5 million words, collected from newspaper articles, periodicals, and paragraphs of literature, was processed in order to select the utterances-sentences needed for producing the speech database and to achieve a reasonable phonetic coverage. The broad coverage and contents of the selected utterances-sentences of the database text corpus collected from different domains and writing styles - makes this database appropriate for various application domains. The database, recorded in audio studio, consists of approximately 3,000 phonetically balanced Modern Greek utterances corresponding to approximately four hours of speech. Annotation of the Vergina speech database was performed using task-specific tools, which are based on a hidden Markov model (HMM) segmentation method, and then manual inspection and corrections were performed.

\subsubsection{Design of the Vergina database}

The most crucial requirement in the design phase of a speech database for speech synthesis is the adequate phonetic coverage of the selected text corpus. In corpusbased speech synthesis, the quality of the output is highly correlated to the coverage of the database. In detail, it is necessary to include most of the contextual segmental variants in the database along with as more phonetic transitions as possible (diphones), and thus compensate for the co-articulation phenomenon in speech (Kominek and Black, 2003). A text corpus fulfilling this particular condition is characterized as phonetically rich (Black and Taylor, 1997). In the designing phase of the Vergina speech database, this requirement was attained by utilizing an automatic selection of text data from a large corpus.

Furthermore, the design of the database was guided by the needs of building a Greek unit-selection corpus-based voice operating with phone sized units as well as by the needs of a HMM-based voice. Even though perfect quality open-domain synthesis is not yet possible (Kominek and Black, 2003), an attempt was made not to restrict the database to a specific narrow domain. This was achieved by designing the contents in such a way, so that a number of dissimilar domains are covered in the recordings. To implement this intention, we included in the database text collected from different domains and sources such as newspapers, periodicals, and literature. For that purpose the prompt sentences were designed through the following steps: (i) 
selecting a source text corpus to represent the target domains, (ii) analyzing the source text corpus to obtain the unit statistics and finally (iii) selecting appropriate prompt sentences from the source text.

In the first step, a large amount of textual material, approximately 5 million words, was collected from articles in newspapers (approximately 2.2 million words) and periodicals (approximately 1.4 million words) as well as from excerpts from the literature (approximately 1.4 million words). The entire text corpus consists of approximately 280 thousand utterances. In the second step, a subset of utterances was produced, by using a Festvox (Black and Lenzo, 2000) script and a Modern Greek diphone TTS based on the Festival Speech Synthesis framework (Black and Taylor, 1997). Specifically, this script applies a filter on the entire text corpus, in order to select a subset of sentences of length between 5 and 15 words (Kominek and Black, 2003), which are easily read. This procedure resulted in a subset of approximately 95 thousand utterances (sentences, paragraphs) of an appropriate length, which are easily pronounceable. Finally in the third step, this subset was further processed using the dataset-select Festvox procedure (Black and Lenzo, 2000), which is based on a greedy search algorithm and leads to the final subset of sentences. The criterion for selection is the sentences to have the best diphone coverage - with the maximum number of diphones and the maximum occurrences of these diphones. An advantage of the Greek language is that the stress is clearly defined in the text (by the stress symbol) over every stressed vowel (i.e. $\alpha / \alpha, \varepsilon / \varepsilon, i / l$, etc.). Thus stressed vowels are represented with unique phonetic symbols. Accounting for this norm in the Modern Greek language and for the fact that stressed syllables play a very important role in the language, we used distinctive representations for the vowels of the stressed and unstressed syllables (i.e. $\mathrm{A} / \mathrm{a}, \mathrm{E} / \mathrm{e}, \mathrm{I} / \mathrm{i}$, etc).

The above mentioned steps resulted in a set of approximately 3,000 sentences. This set corresponds to approximately 23,500 words $-8,000$ unique words - and to approximately 60,000 and 127,000 syllables and phones respectively.

Figure 2.1 and Tables 2.2 and 2.3 show structural information of the Vergina speech database. In particular, in Fig. 2.1 the number of words per sentence is presented. In Table 2.2 the twenty most frequent words of the database are presented along with the number of their occurrences and the pronunciation of the words. In Table 2.3 the twenty most frequent diphones of the database are presented along with the number of their occurrences. 


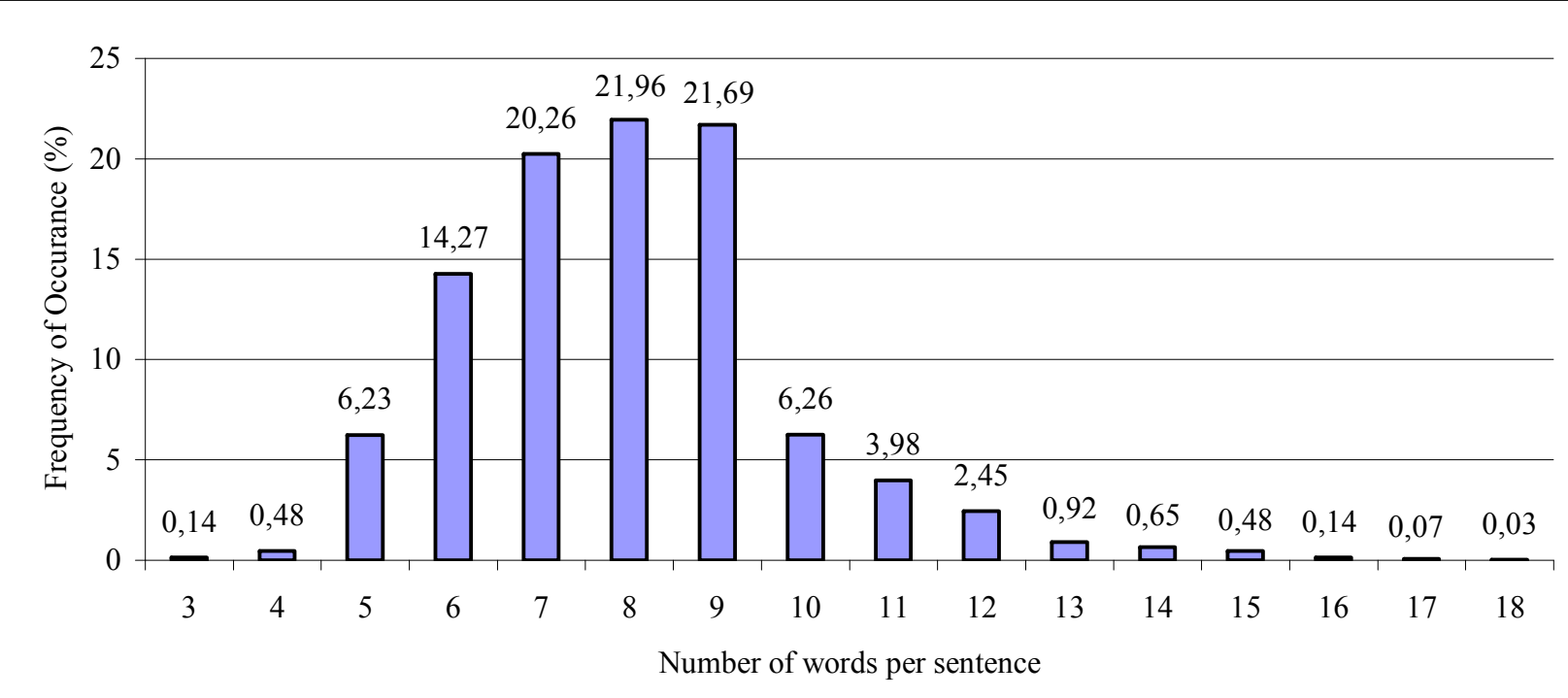

Fig. 2.1. The number of words per sentence. 
Table 2.2. The twenty most frequently occurred words in the database along with their pronunciation.

\begin{tabular}{ccc|ccc}
\hline Word & Pron. & $\begin{array}{c}\text { Num. of } \\
\text { Occur. }\end{array}$ & Word & Pron. & $\begin{array}{c}\text { Num. of } \\
\text { Occur. }\end{array}$ \\
\hline$\kappa \alpha l$ & $\mathrm{Ke}$ & 586 & $\tau \alpha$ & ta & 279 \\
$\tau o$ & to & 566 & $\gamma l \alpha$ & $\mathrm{Ya}$ & 258 \\
$v \alpha$ & $\mathrm{na}$ & 545 & $o l$ & $\mathrm{i}$ & 249 \\
$\eta$ & $\mathrm{i}$ & 502 & $\theta \alpha$ & $\mathrm{Qa}$ & 245 \\
$\tau O v$ & $\mathrm{tu}$ & 470 & $\mu \varepsilon$ & $\mathrm{me}$ & 227 \\
$\varepsilon i v \alpha l$ & $\mathrm{Ine}$ & 428 & $\alpha \pi o ́$ & $\mathrm{apO}$ & 225 \\
$o$ & $\mathrm{o}$ & 369 & $\sigma \varepsilon$ & $\mathrm{se}$ & 180 \\
$\tau \eta v$ & tin & 315 & $\sigma \tau o$ & $\mathrm{sto}$ & 179 \\
$\tau \eta \varsigma$ & tis & 311 & $\pi o v$ & $\mathrm{pu}$ & 171 \\
$\delta \varepsilon v$ & Den & 300 & $\tau \omega v$ & ton & 167 \\
\hline
\end{tabular}

Table 2.3. The twenty most frequently occurred diphones in the database.

\begin{tabular}{cc|cc}
\hline Diphone & $\begin{array}{c}\text { Num. of } \\
\text { Occur. }\end{array}$ & Diphone & $\begin{array}{c}\text { Num. of } \\
\text { Occur. }\end{array}$ \\
\hline$t-i$ & 2575 & $a-s$ & 1232 \\
$i-s$ & 2118 & $t-a$ & 1132 \\
$s-t$ & 1962 & $a-p$ & 1061 \\
$s-i$ & 1658 & $o-n$ & 1035 \\
$t-o$ & 1578 & $n-e$ & 1030 \\
$n-a$ & 1542 & $e-t$ & 1013 \\
$a-t$ & 1354 & $r-i$ & 960 \\
$i-n$ & 1348 & $t-e$ & 958 \\
$a-n$ & 1288 & $i-a$ & 940 \\
$o-s$ & 1269 & $m-e$ & 932 \\
\hline
\end{tabular}

The phone-set used in the database is a modification of the SAMPA (Wells, 1997) phonetic alphabet for Greek. The phone-set consisted of 39 phones plus the silent (pau) was adopted. These forty phones define eight classes as follows:

- Vowels

○ Stressed Vowels: /A/, /E/, /I/, /O/, /U/,

○ Unstressed Vowels: /a/, /e/, /i/, /o/, /u/,

- Consonants

- Affricates: /c/, /j/,

○ Fricatives: /D/, /f/, /Q/, /s/, /v/, /x/, /X/, /y/, /Y/, /z/,

○ Liquids: /1/, /L/, /r/,

- Nasals: $/ \mathrm{m} /, / \mathrm{n} /, / \mathrm{N} /, / \mathrm{h} /$,

- Plosives: /b/, /d/, /g/, /G/, /k/, /K/, /ks/, /p/, /t/, /w/,

○ Silence: /pau/. 
The percentage of the diphone coverage for Vergina database is nearly $75 \%$. This percentage is derived based on the consideration that, in theory, the maximum number of the diphones is $1599=40 \times 40-1$. However, the real percentage is even higher since the realizable diphones in Greek language are less than 1599. In Table 2.4 each phone of the Vergina speech database phone-set along with the respective symbol of the IPA alphabet is presented.

Table 2.4. Vergina Speech Database phone set along with the respective symbols of the IPA alphabet.

\begin{tabular}{cc|cc|cc}
\hline $\begin{array}{c}\text { Vergina } \\
\text { Phoneset }\end{array}$ & $\begin{array}{c}\text { IPA } \\
\text { Alphabet }\end{array}$ & $\begin{array}{c}\text { Vergina } \\
\text { Phoneset }\end{array}$ & $\begin{array}{c}\text { IPA } \\
\text { Alphabet }\end{array}$ & $\begin{array}{c}\text { Vergina } \\
\text { Phoneset }\end{array}$ & $\begin{array}{c}\text { IP } \boldsymbol{A l p h} \\
\text { Phabet }\end{array}$ \\
\hline$A$ & $\mathrm{a}$ & $i$ & $\mathrm{i}$ & $Q$ & $\theta$ \\
$a$ & $\mathrm{a}$ & $j$ & $\mathrm{~d} 3$ & $r$ & $\mathrm{r}$ \\
$b$ & $\mathrm{~b}$ & $K$ & $\mathrm{c}$ & $s$ & $\mathrm{~s}$ \\
$c$ & $\mathrm{f}$ & $k$ & $\mathrm{k}$ & $t$ & $\mathrm{t}$ \\
$D$ & $\mathrm{~d}$ & $k s$ & $\mathrm{ks}$ & $U$ & $\mathrm{u}$ \\
$d$ & $\mathrm{~d}$ & $L$ & $\kappa$ & $u$ & $\mathrm{u}$ \\
$E$ & $\mathrm{e}$ & $l$ & $\mathrm{l}$ & $v$ & $\mathrm{v}$ \\
$e$ & $\mathrm{e}$ & $m$ & $\mathrm{~m}$ & $w$ & $\mathrm{ps}$ \\
$f$ & $\mathrm{f}$ & $N$ & $\mathrm{n}$ & $X$ & $\mathrm{c}$ \\
$G$ & $\mathrm{~J}$ & $n$ & $\mathrm{n}$ & $x$ & $\mathrm{x}$ \\
$g$ & $\mathrm{~g}$ & $O$ & $\mathrm{o}$ & $Y$ & $\mathrm{j}$ \\
$h$ & $\mathrm{y}$ & $o$ & $\mathrm{o}$ & $y$ & $\mathrm{\gamma}$ \\
$I$ & $\mathrm{i}$ & $p$ & $\mathrm{p}$ & $z$ & $\mathrm{Z}$ \\
\hline
\end{tabular}

\subsubsection{Recordings of the Vergina database}

The Vergina database has been recorded in the audio studio located in the premises of the Artificial Intelligence Group at the University of Patras. The studio walls (floating screed) are $12 \mathrm{~cm}$ thick filled with glass-wool material. Heavy curtains and carpets are installed on the inside area as absorbent material.

The female voice talent, a native Greek speaker, being recorded was sitting in front of a personal computer with her mouth 10 to $20 \mathrm{~cm}$ away from the microphone. A pop filter was installed between the speaker and the microphone to reduce the force of airflow to the microphone. Furthermore, a high fidelity audio capture card was used, and the audio was sampled with sampling rate of $44.1 \mathrm{kHz}$ and a resolution of 16 bit per sample. 
The talent used a customized graphical user interface (GUI) to process the utterances. This GUI consists of one box showing the utterance to be captured and four buttons; two buttons for starting and stop recording, a "play" button allowing the talent validating the recording and a "next" button for saving the current recording/proceeding to the next utterance to be recorded.

Due to the large amount of recordings, the database collection campaign had duration of two weeks. Consequently, in order to reduce the unevenness which could result due to the multiple recording sessions, the speaker was instructed to speak in a neutral voice with minimal inflection. In total, the database was recorded in fifteen sessions, each one with length of approximately two hours. The Vergina speech database consists of approximately 3,000 sentences corresponding to approximately four hours of high quality speech.

After the end of the recording campaign, all recordings were checked in order to identify and re-record the misspellings and other mistakes in the database. The mistaken recordings, which were approximately the $10 \%$ of the whole database, were recorded again in purposely-planed additional recording session.

\subsubsection{Annotations of the Vergina database}

Annotations were semi-automatically created utilizing task-specific tools; based on a hidden Markov model (HMM) segmentation method (Mporas et al., 2008). In detail, we used the word-level prompts of each speech recording file to compute the phonetic-level annotation as well as the positions of the phonetic transitions.

With the use of a pronunciation dictionary we converted the word level transcriptions to the corresponding phone sequences. The pronunciation dictionary consisted of the phonetic representation of all the pronounced words of the prompts, using a set of forty phones of the Greek language. As described in Section 2.2.1, the phone-set used for the annotations of Vergina speech database is a modification of the SAMPA (Wells, 1997) phonetic alphabet for Greek. The phonetic representation of each word of the dictionary was made manually by expert phoneticians.

After producing the phonetic transcription for each speech waveform of the database we estimated the phonetic boundary positions by time-aligning the phone sequences with HMM phone models. In order to accurately estimate the phonetic 
transition positions we used the hybrid-HMM method of Mporas et al. (2008). In this method, for each phone an HMM model is initially constructed by embedded training (Young et al., 2006) of the corresponding HMMs. The resulting initial set of HMM models is time-aligned against the phonetic sequences in order to produce a first estimation of the phonetic boundaries. These boundaries are in turn used to train isolated-unit models (Young et al., 2006), which in turn are time-aligned to produce a refined, i.e. more accurate, estimation of the phonetic boundary positions. This procedure is iteratively repeated until the average refinement of the boundary positions reaches a predefined threshold.

Here, we utilized context-independent HMM phone models. The parameterization of the speech recordings was performed using the Mel frequency cepstral coefficients (Davis and Mermelstein, 1980), within a 20 millisecond sliding Hamming window, with step 5 milliseconds.

Except for the word-level and phone-level segmentation the database was annotated in syllable-level. Furthermore after the HMM-based segmentation, effort for manual inspection and correction of the automatic annotations, concerning the full size of the database, took place for improving the automatic annotation on the phonelevel and on the syllable-level and thus improving the quality of the derivative speech voice (synthetic speech). The manual inspection and correction of the automatic annotations of the speech database was made using the PRAAT software (Boersma and Weenink, 2008). The most important criterion for the hand-correction of the boundaries of each phone, and subsequently of each syllable and word was the listening perception of the speech signal, along with the visual observation of it and its spectrum.

In Table 2.5 the mean durations, the standard deviations and the number of occurrences of all the phones of Vergina speech database after the manual correction of the automatic segmentation are presented. 
Table 2.5. Mean duration, standard deviation and number of occurrences of the phones of Vergina speech database.

\begin{tabular}{cccc|cccc}
\hline Phone & $\begin{array}{c}\text { Mean } \\
\text { Duration }\end{array}$ & $\begin{array}{c}\text { Standard } \\
\text { Deviation }\end{array}$ & $\begin{array}{c}\text { Number of } \\
\text { Occurrences }\end{array}$ & Phone & $\begin{array}{c}\text { Mean } \\
\text { Duration }\end{array}$ & $\begin{array}{c}\text { Standard } \\
\text { Deviation }\end{array}$ & $\begin{array}{c}\text { Number of } \\
\text { Occurrences }\end{array}$ \\
\hline$A$ & 113.35 & 26.86 & 2911 & $m$ & 76.39 & 15.39 & 4041 \\
$a$ & 64.27 & 21.90 & 11080 & $N$ & 97.31 & 25.61 & 158 \\
$b$ & 89.95 & 24.75 & 290 & $n$ & 64.21 & 18.26 & 7367 \\
$c$ & 123.82 & 29.01 & 165 & $O$ & 100.40 & 29.73 & 3485 \\
$D$ & 75.47 & 20.04 & 2194 & $o$ & 60.20 & 21.20 & 7960 \\
$d$ & 89.69 & 21.20 & 888 & $p$ & 84.62 & 23.63 & 4648 \\
$E$ & 99.65 & 26.69 & 2914 & $Q$ & 94.57 & 23.65 & 1537 \\
$e$ & 57.87 & 18.05 & 8221 & $r$ & 44.42 & 12.98 & 5676 \\
$f$ & 92.63 & 26.82 & 1715 & $s$ & 84.58 & 30.10 & 9120 \\
$G$ & 114.72 & 27.33 & 64 & $t$ & 76.61 & 20.77 & 8955 \\
$g$ & 102.15 & 28.29 & 323 & $U$ & 95.12 & 31.45 & 648 \\
$h$ & 59.43 & 22.58 & 156 & $u$ & 49.38 & 21.90 & 2237 \\
$I$ & 91.44 & 31.80 & 5474 & $v$ & 82.16 & 21.31 & 1090 \\
$i$ & 56.14 & 24.42 & 12860 & $w$ & 152.16 & 25.71 & 257 \\
$j$ & 123.99 & 36.98 & 129 & $X$ & 118.02 & 29.82 & 680 \\
$K$ & 103.73 & 29.33 & 2002 & $x$ & 101.15 & 22.38 & 1014 \\
$k$ & 86.47 & 22.43 & 2698 & $Y$ & 98.65 & 29.07 & 958 \\
$k s$ & 153.26 & 26.34 & 618 & $y$ & 70.45 & 17.93 & 1042 \\
$L$ & 95.38 & 30.37 & 160 & $z$ & 82.73 & 20.71 & 893 \\
$l$ & 77.08 & 16.70 & 3184 & $p a u$ & 270.09 & 188.43 & 16673 \\
\hline
\end{tabular}

\subsection{Unit-selection speech synthesis system}

The unit-selection speech synthesis system which is presented in this section was developed under the Festival framework (Black and Taylor, 1997; Black and Lenzo, 2000) during this dissertation. In unit-selection speech synthesis, a large speech database containing natural speech of several hours is used in order to synthesize speech. During synthesis time, units (e.g. phones or diphones) are selected from the database and concatenated together to synthesize a new utterance. The database contains hundreds or thousands of instances of each phone or diphone.

Specifically, during synthesis time, the frontend (cf. Section 1.3) of the speech synthesis system processes the input text and produces a target utterance along with information concerning the phonetic representation of the text, phone sequence, linguistic information concerning the phones, and prosodic information concerning the pitch contour, the duration of phones, the determination of accents, the phrase boundaries, etc. Consequently this information is used for selecting the units from the database which best match the target units and concatenating them. 


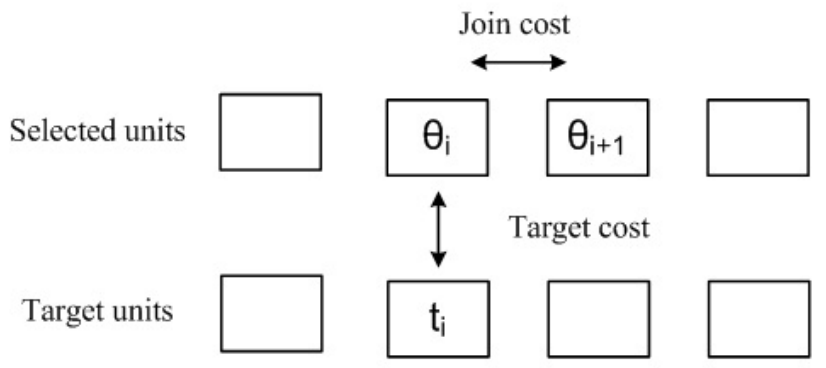

Fig. 2.2. Diagram of the target and join cost functions.

For measuring which units best match the target units, two cost functions are used, the target cost and the join cost (see Fig. 2.2) (Hunt and Black, 1996). The target cost measures the degree to which the candidate unit matches the target unit. The target cost is measured in terms of constituent phones in a syllable, word or phrase and is computed based on linguistic, contextual and prosodic information as mentioned above. On the other hand, the join cost measures how well two consecutive units will join together producing the least possible distortion in the concatenation points. The join cost is based on the use of acoustic features such as the Mel-frequency cepstral coefficients (MFCC). All the features participate in the cost functions with a weighing factor. These weights are determined during training phase. The weights are calculated so as to minimize the difference between a natural utterances belonging to the database and the respective synthetic utterance synthesized by the system given the specifications of the target utterance.

Since the target and join costs are computed for each target unit of the utterance to be synthesized, the Viterbi algorithm is used for searching the best sequence of units that minimises the total cost (see Fig. 2.3). Finally these units are concatenated together to produce the synthetic speech corresponding to the input text. 


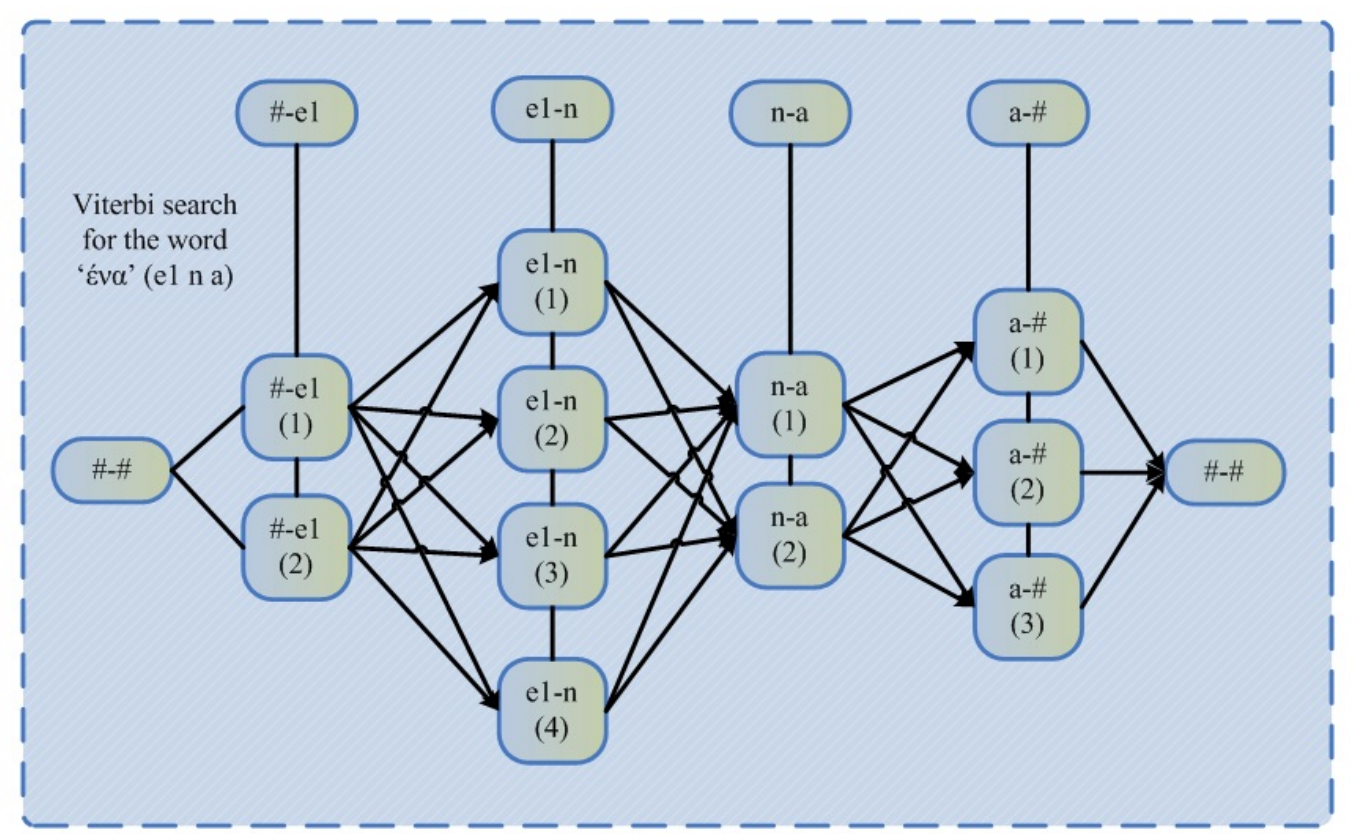

Fig. 2.3. Viterbi search for the sequence of diphones of the word "Éva" (one).

\subsection{HMM-based speech synthesis system}

The HMM-based speech synthesis system which is presented in this section was developed under the HTS (Zen et al., 2007c) framework (see Fig 2.4). In HMM-based parametric speech synthesis, the spectrum, pitch and duration of natural speech are simultaneously modelled in a unified framework of HMMs (Yoshimura et al., 1999). Specifically, in the training phase, the spectrum (Mel-based cepstral coefficients) is modelled by continuous probability distribution HMMs, the pitch $(\operatorname{logF} 0)$ is modelled by multi-space probability distribution HMMs and the state durations by multi-space Gaussian distribution. Multi-space distribution models are used in order to overcome the problem of non continuous pitch values in the parts of unvoiced speech (Tokuda et al., 1999). Context-dependent decisions trees are used for clustering independently the distributions of the spectral, pitch and duration parameters.

In synthesis phase, the input text is converted to a context-dependent phoneme sequence. A sentence HMM is created by concatenating phoneme HMMs based on the phoneme sequence. The decision trees are used in order to select the appropriate HMMs (see Fig. 2.5). Consequently speech parameters vector sequences are generated by the concatenated phoneme HMMs using a speech parameter generation 
algorithm (Tokuda et al., 2000). Finally the synthetic speech is generated through the Mel log spectral approximation (MLSA) filter (Fukada et al., 1992).

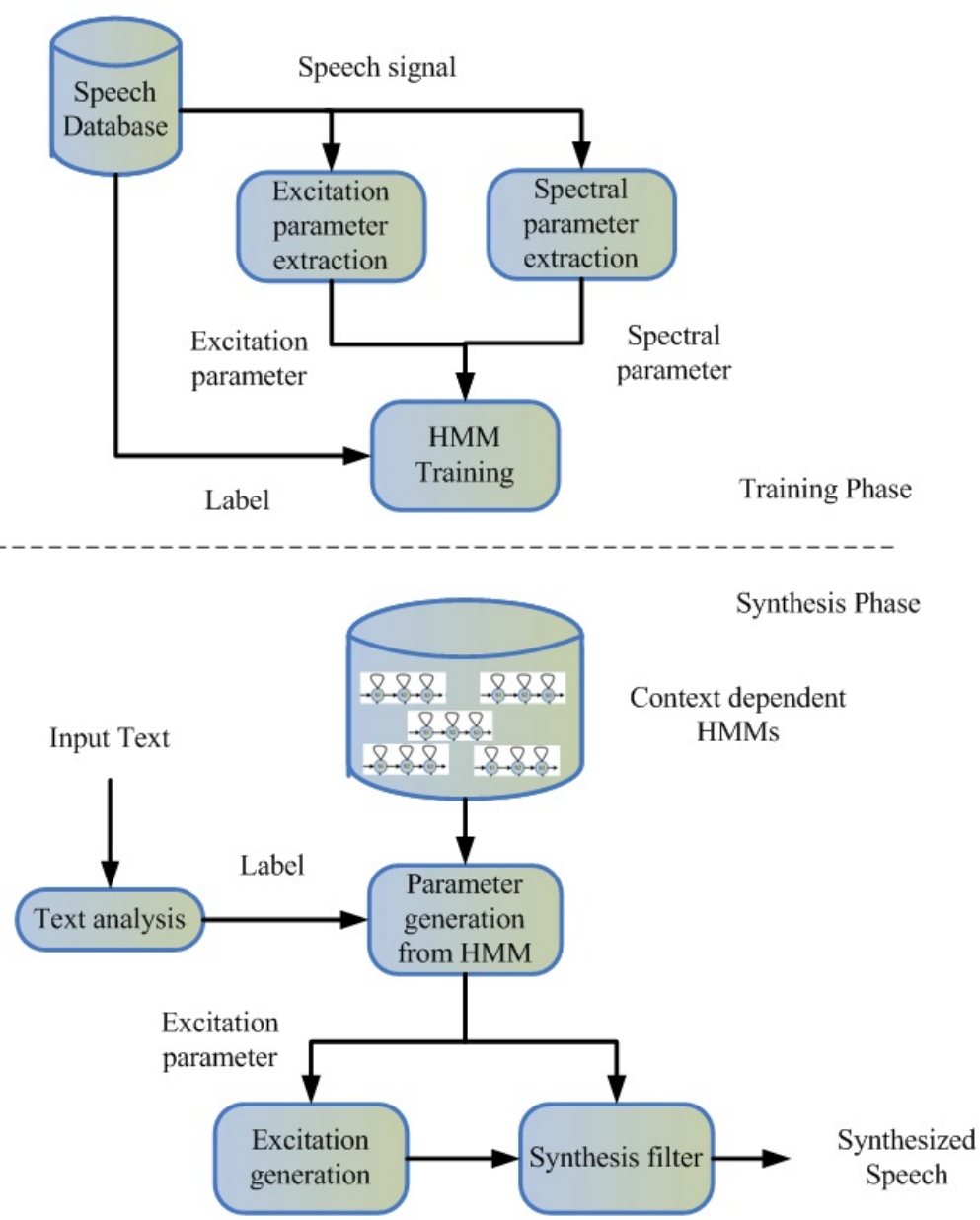

Fig. 2.4. Overview of a typical HMM-based speech synthesis system (Zen et al., 2007c).

e1

$\mathrm{n}$

a

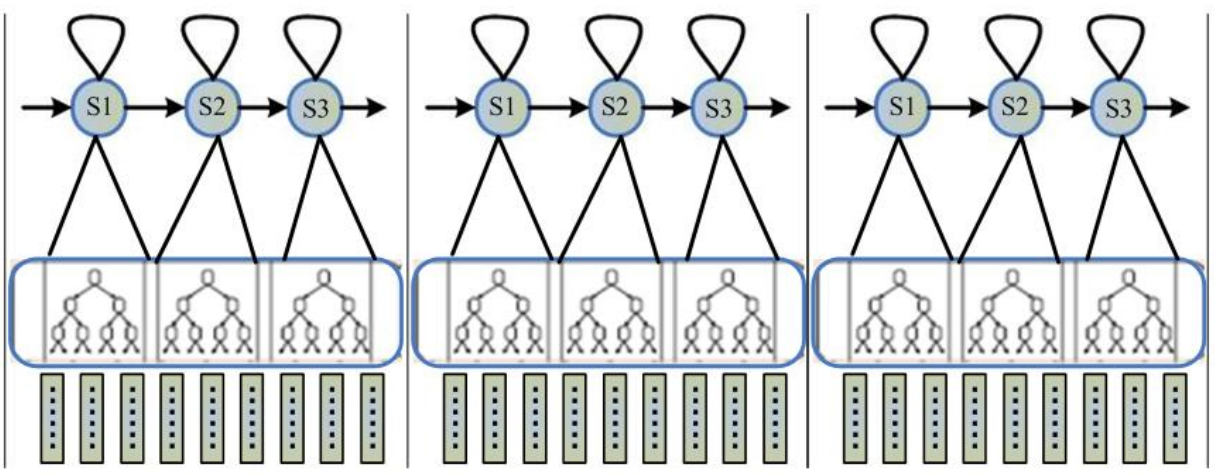

Fig. 2.5. Concatenation of context-dependent HMMs for generating the word "Éva" (one). 
Nonetheless, an inconsistency is raised in this procedure since state duration models are explicitly used during the synthesis phase without being incorporated in the training phase. This drawback of HMMs can be overcome with the use of the HSMMs (Zen et al., 2004). HSMMs are characterized by their ability to incorporate the explicit modelling of state durations not only in the synthesis phase as HMMs do, but also in the training phase of the HSMM-based speech synthesis systems improving the naturalness of synthetic speech (Zen et al., 2004).

Our HMM-based speech synthesis system follows the framework described above with the proper adaptation to Greek language and the characteristics governing it. As described above in Section 2.2.1, the phone-set which was used is a modification of the SAMPA (Wells, 1997) phonetic alphabet for Greek. The phone-set consisted of 39 phones (phonemes) plus the silent (pau) was adopted, divided in 29 consonants and allophones, 5 stress and 5 unstressed vowels. The reasoning behind the choice to use separate symbols for stressed and unstressed vowels is the advantage in Greek language to define the stress in the plain text or by simple rules in the grapheme-tophoneme conversion.

The set of the context factors extracted from the database for training the HMMs and the decision trees are presented next:

- Phonetic level

i. The phoneme identity along with the identities of the two previous and two following phonemes.

ii. The position of the phoneme in the syllable.

- Syllable level

i. The number of phonemes in the syllable along with the respective information for the previous and following syllables.

ii. The position of the syllable in the word and in the phrase.

iii. Stressed/unstressed syllable information.

iv. The number of vowels in the syllable.

v. The number of syllables since the previous and till the following stressed syllable.

- Word level

i. The number of syllables in the word along with the respective information for the previous and following word. 
ii. The position of the word in the phrase.

iii. The part-of-speech of the word.

- Phrase level

i. The number of syllables in the phrase.

ii. The number of words in the phrase.

iii. The number of phrases in the sentence.

iv. The position of the phrase in the sentence.

The speech signals of the database were down-sampled to the frequency of $16 \mathrm{kHz}$ and a resolution of 16 bit per sample. 5-state left-to-right with no-skip HSMMs were used. The parameters were extracted using a $25 \mathrm{msec}$ Hamming-windowed frame length with a $5 \mathrm{msec}$ frame shift. The feature vector consisted of $25 \mathrm{Mel}$-frequency cepstral coefficients (MFCCs), including zeroth coefficient, and logarithm of fundamental frequency $(\log \mathrm{F} 0)$. Moreover, both dynamic (delta) and acceleration (delta-delta) coefficients were used both for spectrum (MFCC) and pith (logF0) representation.

\subsection{Conclusions}

In this chapter, the design and development of Vergina speech database, which was developed in support of research and development of corpus-based unit selection and statistical parametric speech synthesis systems for Modern Greek language was presented. Furthermore, a unit-selection corpus-based and a HMM-based statistical parametric speech synthesis systems developed during this $\mathrm{PhD}$ dissertation were presented. 


\section{Chapter 3}

\section{INTRODUCTION TO PROSODY MODELLING}

In this chapter, a brief overview of the phone duration modelling approaches developed over the years is presented. Specifically an introduction to rule-based and data-driven methods is presented along with an overview over the features which have been used in phone duration modelling over the last decade.

In Text-to-Speech synthesis (TTS) there are two major issues concerning the quality of the synthetic speech, namely the intelligibility and the naturalness (Dutoit, 1997; Klatt, 1987). The former refers to the capability of a synthesized word or phrase to be comprehended by the average listener. The latter represents how close to the human natural speech, the synthetic speech is perceived. One of the most important factors for achieving intelligibility and naturalness in synthetic speech is the accurate modelling of prosody. The prosody controls the phone duration, fundamental frequency and loudness of speech. The phone duration controls the rhythm and the tempo of the speech (Yamagishi et al. 2008). Thus, flattening the prosody in a speech waveform would result in a monotonous and without rhythm speech, sounding unnatural and unpleasant.

Various studies concerning phone duration modelling (Bartkova and Sorin, 1987; Bell et al., 2003; Carlson and Granstrom, 1986; Chung, 2002; Crustal and House, 1988; Epitropakis et al., 1993; Goubanova and King, 2008; Gregory et al., 2001; Iwahashi and Sagisaka, 2000; Klatt, 1976; Kohler, 1988; Krishna and Murthy, 2004; 
Lazaridis et al., 2007; Lazaridis et al., 2011; Lee and Oh, 1999a; Lee and Oh, 1999b; Mobius and Santen, 1996; Riley, 1992; van Santen, 1994; Simoes, 1990; Takeda et al., 1989; Teixeira and Freitas, 2003) have been made over the last decades. The existing phone duration modelling methods are divided in two major categories: rulebased (Bartkova and Sorin, 1987; Carlson and Granstrom, 1986; Epitropakis et al., 1993; Klatt, 1976; Kohler, 1988; Simoes, 1990) and data-driven methods (Chung, 2002; Goubanova and King, 2008; Iwahashi and Sagisaka, 2000; Krishna and Murthy, 2004; Lazaridis et al., 2007; Lazaridis et al., 2011; Lee and Oh, 1999a; Lee and Oh, 1999b; Mobius and Santen, 1996; Riley, 1992; van Santen, 1994; Takeda et al., 1989; Teixeira and Freitas, 2003).

\subsection{Rule-based methods}

The rule-based techniques rely on manually produced rules, extracted from experimental studies on large sets of utterances or based on previous knowledge concerning factors which affect the segmental durations. Some of the most frequently studied and used factors in duration modelling are the identity of the neighbouring segments (previous or next), the position of the segment in the syllable or in the word, the phrase boundaries and many other factors. Expert phoneticians are required for studying these factors and extract the rules which are used for determining the duration of the segments.

Dennis Klatt (1979) introduced one of the first and most well known attempts in the field of rule-based segmental duration modelling. The derived rules, by analyzing a phonetically balanced set of sentences, were used in order to predict segmental duration. These rules were based on linguistic information such as phonological, morphological and syntactic factors. Initially a set of intrinsic values was assigned on each phone, which was modified each time according to the extracted rules, such as the example given in eq. (3.1) from Klatt (1976):

$$
D U R=M I N D U R+\frac{(I N H D U R-M I N D U R) * P R C N T}{100}
$$

where INHDUR is the inherent (intrinsic) duration of each segment, MINDUR is the minimum duration which is assigned to each segment in order to ensure that the 
duration of the segment will never be too short and finally, PRCNT is the shortening percentage determined by the applied rules (Klatt, 1976).

Similar models were developed in other languages, such as: French (Bartkova and Sorin, 1987), Swedish (Carlson and Granstrom, 1986), German (Kohler, 1988) and Greek (Epitropakis et al., 2003), and varieties, such as: American English (Allen et al., 1987; Olive and Liberman, 1985) and Brazilian Portuguese (Simoes, 1990). The major drawback of the rule-based approaches is the difficulty to represent and manually tune all the linguistic factors which influence the segmental duration in speech. Thus, in order to collect all the appropriate (or even adequate amount of) rules, long-term devotion to this task becomes crucial and mandatory (Klatt, 1987). Therefore, the rule-based duration models are restricted to controlled experiments, where only a limited number of contextual factors are involved, in order to be able to deduce the interaction among these factors and extract these rules (Campbell, 1992).

\subsection{Data-driven methods}

Data-driven techniques for the task of phone duration modelling were developed after the construction of large databases (Kominek and Black, 2003). These approaches are based either on statistical methods or artificial neural network (ANN) based techniques. Data-driven approaches automatically produce phonetic rules and construct duration models using large speech corpora, overcoming the problem of manually extracting rules. Their main advantage is that this process does not depend so much on manual labour of phoneticians as the rule-based techniques do, where the knowledge and expertise of phoneticians is mandatory for producing the rules.

In the phone duration modelling task various statistical methods have been applied over the last years such as, linear regression (LR) (Takeda et al., 1989) models, decisions tree-based models (Krishna et al., 2004; Mobius and Santen, 1996; Riley, 1992) and sums-of-products (SOP) (Febrer et al., 1998; van Santen, 1992). Furthermore, artificial neural networks (Campbell, 1992; Cordoba et al., 2001), Bayesian networks models (Chien et al., 2003; Goubanova and King, 2008) and lazylearning algorithms (Lazaridis et al., 2007) have also been introduced on the phone 
duration modelling task. In the following, some of the most frequently and successfully used methods for phone duration modelling are briefly mentioned.

The linear regression (LR) (Iwahashi and Sagisaka, 2000; Lazaridis et al., 2007; Takeda et al., 1989; Yamagishi et al., 2008) models are based on the assumption that there is linear independence among the features which affect the segmental duration. Specifically, the features are weighted in a linear combination creating a prediction function. In (Lazaridis et al., 2007), on a Modern Greek speech database, it was shown that a linear regression model outperformed classification and regression tree (CART) and instance-based (IBK) models in terms of root mean square error (RMSE), achieving duration prediction accuracy of $25.5 \mathrm{~ms}$, against $26 \mathrm{~ms}$ for the CART model and $27.5 \mathrm{~ms}$ for the IBK model, respectively. On the other hand, decision tree-based models, and in particular the CART models (Chung, 2002; Goubanova and King, 2008; Iwahashi and Sagisaka, 2000; Krishna and Murthy, 2004; Lazaridis et al., 2007; Lee and Oh, 1999a; Riley, 1992; Yamagishi et al., 2008), can represent the dependencies among the features but cannot put constraints of linear independency for reliable predictions (Iwahashi and Sagisaka, 2000). Model trees is another tree-based technique incorporating both linear regression and regression trees overcoming the drawbacks of each of these two methods (Iwahashi and Sagisaka, 2000; Yamagishi et al., 2008). Specifically when the training data related to a specific feature are few, model trees incorporate the linear independence constrains between this feature and the others, using a small number of free parameters as the linear regression models do. On the other hand, when the amount of training data related to a specific feature is large, the model trees represent the dependence between this feature and the others using a large number of parameters as the regression trees do (Iwahashi and Sagisaka, 2000). Even though decision trees manage to build models which fit the training data well, there is no certainty that during the operational phase the unseen data will be predicted properly. This statement is based on the fact that these models lack the ability of interpolation and cannot handle sparse data, which deteriorates their performance (Rao and Yegnanarayana, 2007; Goubanova and King, 2008; Riley, 1992). In (Yamagishi et al., 2008), on two Japanese (male and female) and one American English (male) databases model trees outperformed the respective CART models in both vowel and consonant categories. Iwamagishi and Sagisaka in (2000) also presented that model trees outperformed CART model in a Received 
Pronunciation (RP) English database enforcing the advantage of model trees over regression trees.

In addition, artificial neural networks, such as the feed-forward neural networks (Teixeira and Freitas, 2003), have been used for the needs of phone duration modelling. It is well known that neural networks have the ability of generalizing and capturing the relationship between the input and the output patterns, which makes them appropriate for the task of phone duration modelling (Rao and Yegnanarayana, 2007; Yegnanarayana, 1999). Furthermore, an additional advantage of neural networks is their ability to cope with missing data (Yegnanarayana, 1999).

Bayesian networks models have also been introduced in the phone duration modelling task. These models incorporate a straightforward representation of the problem domain information, and despite their demanding training they were shown to make accurate predictions even when unknown values come across in some features (Goubanova and King, 2008). Moreover, the sums-of-products (SOP) method has been used in phone duration modelling where the segmental duration modelling is based on a sum of factors and their product terms that affect the duration (Chung, 2002; Goubanova and King, 2008; van Santen, 1994). SOP models can be trained using small amount of data and have the ability of interpolation when they come across with rare or unseen feature vectors in comparison to the regression trees. On the other hand, the main drawback of the SOP models lies upon the exponentially growth of the number of possible models when the dimension of the feature vector increases, making expert intuition and the use of heuristic search techniques mandatory for finding the best SOP model (Goubanova and Taylor, 2000; Goubanova and King, 2008). In (Chung, 2002), on a Korean database, a SOP model was reported to achieve accuracy of $32.1 \mathrm{~ms}$ and $28.9 \mathrm{~ms}$ in terms of RMSE on the vowels and consonants respectively, and was outperformed by a CART model which achieved performance of $27.5 \mathrm{~ms}$ and $24.2 \mathrm{~ms}$, respectively. Goubanova and King in (2008) performed a comparison evaluation among Bayesian networks, CART models and SOP models in a British (RP) and an American English database showing that Bayesian networks outperformed both of the other two models in both databases in vowel and consonant categories separately.

Furthermore, instance-based algorithms (Lazaridis et al., 2007) have been used in the phone duration modelling task. In instance-based models the training data are stored during the training phase and a distance function is used during the prediction 
phase in order to determine which member of the training set is closer to the test instance and predict the phone duration. In a resent study (Yamagishi et al., 2008), the gradient tree boosting (GTB) (Friedman, $(2001,2002)$ ) method was introduced in this task as an alternative to the conventional method using regression trees. The GTB algorithm is an additive regression meta-algorithm incorporating multiple regression trees, built in the model iteratively, and consequently taking advantage of them. The advantage of this method lies upon the fact that each new regression tree is constructed using the residuals of the prediction function of the current tree regardless of the structure of the previous trees (Yamagishi et al., 2008). As mentioned above, in (Yamagishi et al., 2008), model trees outperformed the CART models in both categories, vowels and consonants, on the two Japanese (male and female) and the American English (male) databases, though it should be pointed out that the best performance was achieved by the model based on the meta-algorithm GTB, outperforming the model trees, the CART model and the linear regression model. As can be concluded based on the research reported over the years on the phone duration modelling task, the data-driven methods provide a convenient mechanism for overcoming the time consuming labour for the manual extraction of rules that is needed in the rule-based phone duration modelling methods. In the following subsections, the aforementioned algorithms are described.

\subsubsection{Linear regression}

Linear Regression (LR) (Witten and Frank, 2005) algorithm is a prediction algorithm that expresses the class variable as a linear combination of the attributes that are taken into account for constructing the prediction model:

$$
x=w_{0}+w_{1} a_{1}+w_{2} a_{2}+\ldots+w_{m} a_{m}+\ldots+w_{M} a_{M}
$$

where $x$ is the class, $a_{m}$ is the $m t h$ feature value and $w_{m}$ is the $m t h$ weight for $0 \leq m \leq M$. The training data are used to calculate the weights which will be subsequently applied on the feature set, in order to predict the class variable. The predicted value for the $j$ th instance of the training data, where $0 \leq j \leq J$, can be formulated as: 


$$
w_{0} a_{0}^{j}+w_{1} a_{1}^{j}+w_{2} a_{2}^{j}+\ldots+w_{m} a_{m}^{j}+\ldots+w_{M} a_{M}^{j}=\sum_{m=0}^{M} w_{m} a_{m}^{j}
$$

where $a_{0}^{j}$ is always equal to 1 . The LR method is mainly based on the calculation of the $M$ weights, $w_{m}$, in order to minimize the sum of the squares of the differences between the predicted and the actual values over all the training instances. The sum of the squares of the differences is formulated as:

$$
\sum_{j=1}^{J}\left(x^{j}-\sum_{m=0}^{M} w_{m} a_{m}^{j}\right)^{2}
$$

Instead of using all the features, M5' algorithm can be applied for feature selection (Wang and Witten, 1997). During feature selection the attribute with the smallest standardized coefficient is iteratively removed until no improvement is observed in the error estimation. The error estimation is given by the Akaike Information Criterion (AIC) (Akaike, 1974) which can be formulated as:

$$
A I C=2 M+J\left(\ln \left(\frac{2 \pi R_{s}}{J}\right)+1\right)
$$

where $M$ is the number of the features and the $\mathrm{R}_{\mathrm{s}}$ is the residual sum of squares:

$$
R_{S}=\sum_{j=1}^{J}\left(x_{\text {real }}-x_{\text {pred }}\right)^{2}
$$

Here $R_{s}$ is the cumulative square error in respect to the real class values. Smaller values of the AIC correspond to better models.

\subsubsection{Decision trees}

Decision trees are predictive models that create a mapping procedure between observations about an item and the conclusions about its target value (Mitchell, 1997). In these tree structures, leaves represent classes and branches represent conjunctions of features that lead to these classes. Decision trees divide the regions of feature space splitting the tree at each node according to some criteria. In contrast to most decisions trees which choose as splitting criterion the maximization of the information gain, when the class variable is numeric, the minimization of the intra-subset variation in the class values down each branch is more appropriate (Witten and Frank, 2005). One of the most commonly used decision tree is the one based on the M5' algorithm 
(Wang and Witten, 1997). This algorithm can be implemented as model (M5p) and regression (M5p-R) tree.

Specifically, at each node, of a model tree, during the splitting procedure, in order to find the most suitable feature to split that portion $T$ of the training data that reaches at that node, the standard deviation of the class values in portion $T$ is used as a measure of the error at that node. The feature maximizing the error reduction is selected to be used for splitting that specific node. The error reduction is formulated as:

$$
E R=\sigma(T)-\sum_{k} \frac{\left|T_{k}\right|}{|T|} \sigma\left(T_{k}\right)
$$

where $T_{1}, T_{2}, \ldots, T_{k}$, are the sets created during the split at the specific node according to the selected feature. This procedure continuous until the standard deviation of the class values of the instances reaching a node becomes a small fraction of the standard deviation of the original instance set or when only a few instances remain (Witten and Frank, 2005).

After the termination of the splitting procedure in order to reduce the size of the tree which has been built, a pruning process is followed (Witten and Frank, 2005). Firstly, at each node a linear regression model is calculated. Consequently, at each node, an estimation of the expected error for the test data is used, measuring the absolute difference between the predicted value and the class value (real value) and averaging it over all the instances of the training data reaching at that node. The estimate of the expected error is weighted by a factor $(n+v) /(n-v)$, where $n$ is the number of the instances reaching that node and $v$ is the number of the features participating in the linear model at that node (Witten and Frank, 2005). This procedure takes place iteratively for all the nodes of the tree. Finally, a sub-tree is pruned from the tree if the expected estimated error calculated from the linear model at root of the sub-tree is smaller or equal to the one calculated from the sub-tree.

The M5' can be used also as a regression tree (M5p-R) following a respective with the model trees building process. In this case, instead of implementing a linear regression model at each leaf as in the case of the model trees, a class value representing the average value of the instances reaching at that leaf is stored (Quinlan, 1992). 
Furthermore, another widely used decision tree is the Reduced Error Pruning trees (REPTrees) (Kääriäinen and Malinen, 2004). The REPTrees are base on the reduced error pruning technique (Quinlan, 1987). They use a fast pruning algorithm to produce an optimal pruning of a given tree. The REP algorithm works in two phases: First the set of pruning examples $S$ is classified using the given tree $T$ to be pruned. Counters that keep track of the number of examples of each class passing through each node are updated simultaneously. In the second phase, which is a bottom-up pruning phase, these parts of the tree that can be removed without increasing the error of the remaining hypothesis are pruned away. The pruning decisions are based on the node statistics calculated in the top-down classification phase.

\subsubsection{Neural networks}

One of the most important advance of Neural networks is their ability of generalizing and capturing the mapping relationship between the input and output pairs of the data (Yegnanarayana, 1999), making them appropriate even for predicting patterns not presented before. Neural networks are divided in recurrent and feedforward networks depending on whether their architecture contains cycles and their outputs depend on previous and current instances or not. One of the most common and widely used neural network is the Radial basis function neural network (RBFNN) which is described next.

The RBFNN is a feed-forward artificial neural network algorithm with one input layer, one hidden layer and one output layer. The hidden layer consists of a group of $k$ hidden units. Each one of these hidden units corresponds to a local kernel function (Mitchell, 1997). Each unit can represent an instance of the training data - the number of the units, $k$, equals the number of the instances of the training data, $J$ - or especially when $J$ is large, each unit can represent a group of instances. These groups of instances are determined using the $k$-means clustering algorithm (Hartigan, 1975). Each hidden unit is centred at each cluster at the point $X_{u}$ a variance $\sigma_{u}$. These local kernels are implemented with Gaussian functions formulated as:

$$
K_{u}\left(d\left(X_{u}, X_{j}\right)\right)=e^{\frac{1}{2 \sigma_{u}^{2}} d^{2}\left(X_{u}, X_{j}\right)}
$$


where $d\left(X_{u}, X_{j}\right)$ is the distance between the centre of cluster $u, X_{u}$, and the $j t h$ instance $X_{j}$. The output of the RBFNN algorithm is the linear combination of the outputs of the hidden units. The output function is formulated as:

$$
f\left(X_{\text {new }}\right)=\lambda+\sum_{u=1}^{k} w_{u} K_{u}\left(d\left(X_{u}, X_{\text {new }}\right)\right)
$$

where $\lambda$ is a constant value and $w_{u}$, with $l \leq u \leq k$, is the weight corresponding to the respective cluster $u$. The weights $w_{u}$ are adjusted during the training procedure (Mitchell, 1997).

\subsubsection{Lazy-learning algorithms}

This category contains algorithms which defer processing of training data until a query needs to be answered. This usually involves storing the training data in memory and finding relevant data in the database to answer a particular query (Witten and Frank, 2005). Two of the most common used lazy-learning algorithms, instance-based (IBK) and Locally Weighted Learning (LWL) algorithms, are described next.

The IBK algorithm is a $k$-nearest neighbours algorithm (Mitchell, 1997) which during the training phase simply stores the training data. During the operational phase when a new instance is presented, $X_{\text {new }}$, a set of instances related to this one, is used in order to predict the output of the new instance. Usually the Euclidean distance is used in order to find this set of the closest instances to the new instance. When the target class is numerical, as in phone duration modelling, the output of IBK is the mean value of the $k$-nearest neighbouring instances. Instead of using the mean value of the $k$-nearest neighbouring instances, a weighted factor can be used as it was done in our experiments, weighting each of the $k$ neighbours, $X_{i}$, with $1 \leq i \leq k$, according to their distance to the new instance. The output function is formulated as:

$$
f\left(X_{\text {new }}\right)=\frac{\sum_{i=1}^{k} w_{i} f\left(X_{i}\right)}{\sum_{i=1}^{k} w_{i}}
$$


The weight $w_{i}$, is the inverse of the Euclidean distance, $d\left(X_{n e w}, X_{i}\right)$, between the new instance, $X_{\text {new }}$, and the ith instance, $X_{i}$, belonging to the set of the $k$-nearest neighbours of the training data. Furthermore leave-one-out cross-validation was used to select the best value for $k$.

Another well known lazy learning algorithm is the Locally Weighted Learning algorithm (LWL) (Atkeson et al., 1996). LWL is a general algorithm which assigns weights using an instance-based method, e.g. the Linear-NN, which is a nearest neighbour search algorithm, and builds a classifier (i.e. a regression model in the case of phone duration modelling) from the weighted instances. The training instances which are located closer to the prediction point receive usually bigger weights. Furthermore, a distance function is also applied. The data weighting takes place either directly or through weighting an error criterion. Weighting the data can be viewed as replicating relevant instances and discarding the irrelevant ones. Moreover, a weighting function or kernel function is used to calculate a weight for a data point from the distance.

\subsubsection{Sum of Products (SOP)}

The Sum-of-Products (SOP) algorithm belongs to the iterative optimization category of data-driven methods. The basic idea behind this algorithm is that the factors that affect the target class are considered independent to each other and the target variable is computed using a set of basic arithmetic equations, applying additions and multiplications (van Santen, 1992; van Santen, 1994).

$$
f\left(X_{\text {new }}\right)=\sum_{i=1}^{K} \prod_{j=1}^{M} A_{i, j}\left(X_{j}\right)
$$

where $A_{i, j}$ is the parameter factor associating $j$ and the correlation between attributes $i$ and $j$ to the target variable of instance $X_{\text {new }}$.

The advantage of SOP is that it depends on a small number of parameters and a small amount of data in order for a robust model to be trained. However, with the increase of the number of the factors, the number of the possible sum-of-product models grown exponentially making significantly difficult and time consuming the process for building a robust one (van Santen, 1992; van Santen, 1994). 


\subsubsection{Meta-learning algorithms}

Meta-learning algorithms (Vilalta and Drissi, 2002; Witten and Frank, 2005c) are based on the use of classifiers (i.e. regression algorithms in the case of phone duration modelling) converting them into more powerful learners. This happens by applying learning algorithms to meta-data, which are data that provide information about other data managed within an application or environment. Two of the most widely used meta-learning algorithms, the Additive Regression and the Bagging algorithms, are described next.

Additive Regression (Stone, 1985) (Friedman, 2002) is a meta-learning technique which enhances the performance of a regression algorithm. During the training procedure, in each iteration, the additive regression algorithm builds a regression tree using the residuals of the previous tree as training data. Since each tree added to the model fits the training data more closely, this procedure is prone to overfitting. In order to avoid overfitting of the model, instead of subtracting a model's entire prediction to generate the target values (residuals) for the next model, a constant factor (shrinkage) was used. This factor, taking values between 0 and 1 , was used to shrink the predictions by multiplying them with it before subtracting. In this way the fitting of the model to the residuals is reduced, decreasing the chance of overfitting (Witten and Frank, 2005). The regression trees are combined together creating the final prediction function.

Moreover the Bagging algorithm (Breiman, 1996) has been used to model phone duration. In the bagging algorithm, the dataset is split in multi subsets utilizing one regression tree for each one of them. Many of the original instances may be repeated in the resulting training set while others may be left out. The final prediction value is the average of the values predicted by each regression tree.

\subsection{Features used in prosody modelling}

The features used in phone duration modelling are extracted from text and belong to various levels of representation of speech, such as the phonetic, phonological, 
morphological and syntactic level. Some of the most frequently used features in this task are the phone, the number of phones in the syllable, the stress of the syllable, the position of the phone in the syllable, the position of the syllable in the word or in the phrase and the part-of-speech of the word. In addition, in some studies (Iwahashi and Sagisaka, 2000; Mobius and Santen, 1996; Lazaridis et al., 2007) apart from the stress feature, more prosodic features were used in the phone duration modelling task, such as the accent of the syllable, the type of the syllable, the distance to the next or previous accent, the break after the syllable or the distance to the next break or pause. Furthermore, in (Yamagishi et al., 2008; Goubanova and King, 2008; Krishna and Murthy, 2004; Mobius and Santen, 1996; Lazaridis et al., 2007), features concerning the phonetic characteristics of the phones have been incorporated in this task such as the vowel height, the vowel frontness, the lip rounding, the manner of production and the place of articulation, or the number of phones before or after the vowel of the syllable. In other studies (Goubanova and King, 2008; Krishna and Murthy, 2004; Lazaridis et al., 2007; Teixeira and Freitas, 2003) information concerning the neighbouring instances, such as the next or previous phones, the type of the next or previous syllable, the stress or accent of the next or previous syllable, was taken under consideration. In this dissertation, we take advantage of these previous studies and use a large feature vector that includes all the above mentioned features. 


\section{Chapter 4}

\section{PROPOSED PROSODIC MODELS BASED ON MACHINE LEARNING TECHNIQUES}

In this chapter, the three proposed methods in phone duration modelling are presented, evaluated and discussed. All the three proposed techniques are novel in phone duration modelling and have never before been introduced in this task. The first approach, presented in Section 4.1 is a model based on support vector regression (SVR) algorithm. SVR have never before been used in the phone duration modelling task and achieves the lowest error rate among all the other individual models.

Furthermore, a scheme for the fusion of different phone duration models, operating in parallel is proposed in Section 4.2. Specifically, the predictions from a group of dissimilar and independent to each other individual phone duration models are fed to a machine learning algorithm, which reconciles and fuses the outputs of the individual models, yielding more precise phone duration predictions. At the fusion stage, we evaluate the performance of twelve fusion techniques. The proposed fusion scheme, when implemented with SVR (SVR-fusion), contributes to the improvement of the phone duration prediction accuracy over that of the best individual model (SVR) presented in Section 4.1.

Finally, is Section 4.3, a two-stage phone duration modelling scheme, which is applicable to improved prosody modelling in speech synthesis systems is presented. This scheme builds on a number of independent phone duration models performing as feature constructors (FCs) employed in the first stage, and a phone duration model 
(PDM), which operates on an extended feature vector in the second stage. The feature vector, which acts as input to the first stage, consists of numerical and non-numerical linguistic features extracted from text. The extended feature vector is obtained by appending the phone duration predictions estimated by the FCs to the initial feature vector. Experiments validated the advantage of the proposed two-stage scheme, implemented with SVR (SVR-FVE), improving prediction accuracy over the best individual predictor, and to the fusion scheme which just fuses the first-stage (individual models) outputs (SVR-fusion scheme proposed in Section 4.2). The improved accuracy of phone duration modelling contributes to a better control of the prosody, and thus to better quality of the synthetic speech.

\subsection{Prosody modelling with support vector regression}

In this section, a model based on support vector regression (SVR) algorithm is presented and compared with other well known and widely used phone duration models. SVR have never before been used in the phone duration modelling task and achieves the lowest error rate among all the other individual models.

To investigate the practical usefulness of the proposed approach, several individual phone duration models were trained, and evaluated. Here, eight different machine learning algorithms for phone duration modelling are considered, and consequently the outputs of them are fed to the fusion model. These algorithms are well known and have successfully been used over the years, in different modelling tasks. One exception is the support vector regression (SVR) based modelling, which to this end has not been employed on the phone duration modelling task. In brief, for the phone duration modelling task the following algorithms are evaluated: linear regression, decision trees (regression and model trees), and meta-learning algorithms using regression trees as base classifiers. The various individual phone duration models as well as the speech databases used in the experiments and the experimental protocol that was followed, are described in the following subsections. 


\subsubsection{Introduction to support vector regression}

The rapid development and wide-spread use of SVR in statistical learning field is based mainly on the properties of support vector machines, providing models with good generalization performance, the absence of local minima and the sparse representation of solution (Vapnik 1995; Vapnik 1998). In contrast to most traditional methods which implement the Empirical Risk Minimization (ERM) principle (Vapnik 1998), the SVMs implement the Structural Risk Minimization (SRM) principle (Vapnik 1995). The SRM principle seeks to minimize an upper bound of the generalization error rather than minimize the training error, which results in better generalization performance in SVMs. Training an SVM, is equivalent to solving a linearly constrained Quadratic Programming (QP) problem resulting in a unique and global optimal. In addition, SVMs are characterized by the sparse representation of the solution requiring less storage space and time for actual prediction since only the support vectors, which are a subset of the training data, are used in the training procedure.

The basic principle of SVM is the mapping of the training data from the input space onto a higher dimensional feature space using a function $\Phi$, constructing subsequently a separating hyperplane with maximum margin in the feature space. Consider a training set of data, $\left(x_{1}, y_{1}\right),\left(x_{1}, y_{1}\right), \ldots,\left(x_{i}, y_{i}\right), \ldots,\left(x_{N}, y_{N}\right)$, where each $x_{i} \in X \subseteq \mathbb{R}^{n}$, denotes the input space of the sample and has a corresponding target value $y_{i} \in Y \subseteq \mathbb{R}$, for $i=1, \ldots, N$ and $N$ is the total number of the training samples. The regression problem is based on the determination of a linear regression function $f(x)$ that can approximate future values accurately, defined as:

$$
f(x)=w^{T} \Phi\left(x_{i}\right)+b
$$

where $w \subset \mathbb{R}^{n}, b \subset \mathbb{R}$ and $\Phi$ maps the training data to a higher dimensional space. This leads to the following optimization problem:

$$
\underset{w, \xi_{i}, \xi_{i}^{*}}{\arg \min }\left\{\frac{1}{2} w^{T} w+C \sum_{i=1}^{N}\left(\xi_{i}+\xi_{i}^{*}\right)\right\}
$$

subject to: 


$$
\left\{\begin{array}{l}
y_{i}-\left(w^{T} \phi\left(x_{i}\right)+b\right) \leq \varepsilon+\xi_{i}^{*} \\
\left(w^{T} \phi\left(x_{i}\right)+b\right)-y_{i} \leq \varepsilon+\xi_{i} \\
\xi_{i}, \xi_{i}^{*} \geq 0, \iota \in[0, \mathrm{~N}]
\end{array}\right\}
$$

where $\varepsilon \geq 0$ is the maximum deviation allowed during training and $C>0$ is the penalty parameter for excessing allowed deviation. The, $\xi_{i}, \xi_{i}^{*}$ are the slack variables for exceeding the target value more or less than $\varepsilon$, respectively. The first term of eq. (4.2) controls the function capacity and the second term is the empirical error.

Utilizing the method of Lagrange multipliers, $\alpha_{i}, \alpha_{i}^{*}$, a different strategy for finding the maximum or minimum of the function subject to constraints is used, the dual optimization problem is now defined as:

$$
\underset{\varepsilon, a_{i}, a_{i}^{*}}{\arg \min }\left\{\begin{array}{c}
\frac{1}{2} \sum_{i, j}\left(a_{i}-a_{i}^{*}\right)\left(a_{j}-a_{j}^{*}\right)\left\langle x_{i} \cdot x_{j}\right\rangle \\
\varepsilon \sum_{i}\left(a_{i}-a_{i}^{*}\right)-\sum_{i} y_{i}\left(a_{i}-a_{i}^{*}\right)
\end{array}\right\}
$$

subject to:

$$
\left\{\begin{array}{l}
\sum_{i}\left(a_{i}-a_{i}^{*}\right)=0 \\
\left(a_{i}, a_{i}^{*}\right) \in[0, C]
\end{array}\right\}
$$

The main advantage of the dual optimization problem is the easy expandability to a non-linear mapping function. In this case the input data are mapped to a higher dimensionality space by a non-linear function $\Phi$. Consequently the term $\left\langle x_{i} \cdot x_{j}\right\rangle$ of eq. (4.4) is replaced by the kernel function $k\left(x_{i}, x_{j}\right)$. Many kernel functions have been used in non-linear SVR such as polynomial, radial basis function (RBF) or Gaussian functions.

Unfortunately, the size of a SVR model depends on the number of the training samples. Thus for large data sets, algorithm with low memory consumption are desired. The most well known algorithm in this category is the Sequential Minimal Optimization (SMO) algorithm (Kaariainen and Malinen, 2004) applied in regression problems in (Scholkopf and Smola, 2002). Training an SVM corresponds to the solution of a very large quadratic programming (QP) optimization problem. A characteristic of the SMO algorithm is its ability to break the QP problems into a 
sequence of smaller possible QP problems, reducing the amount of memory required in order the SVR to be trained.

\subsubsection{Experimental setup}

The support vector regression (SVR) model (Platt, 1999), which employs the sequential minimal optimization (SMO) algorithm for training a support vector classifier (Smola and Scholkopf, 1998), was used. Many kernel functions have been used in SVR such as the polynomial, the radial basis function (RBF) and the Gaussian functions (Scholkopf and Smola, 2002). In our experiments the RBF kernel was used as mapping function. The $\varepsilon$ and $C$ parameters, where $\varepsilon \geq 0$ is the maximum deviation allowed during training and $C>0$ is the penalty parameter for exceeding the allowed deviation, were set equal to $10^{-3}$ and $10^{-1}$ respectively. This was done after a grid search $\left(\varepsilon=\left\{10^{-1}, 10^{-2}, \ldots, 10^{-5}\right\}, C=\{0.05,0.1,0.3,0.5,0.7,1.0,10,100\}\right)$ on the randomly selected subset of the training data, representing the $20 \%$ of the size of the full training dataset.

\subsubsection{Phone duration models using other algorithms}

Apart from SVR which have never been used before in phone duration modelling, other algorithms which have been reported successfully in the literature in the task of phone duration modelling, were used in our experiments as reported earlier (cf. Section 3.2). In brief, the phone duration modelling algorithms that we consider here are:

(i) The Linear Regression (LR) (Witten and Frank, 2005) algorithm is a prediction algorithm that expresses the class variable as a linear combination of the attributes that are taken into account for constructing the prediction model. In LR the training data are used to calculate the weights which will be subsequently applied on the feature set, in order to predict the class variable. Instead of using all the features, M5' algorithm can be applied for feature selection (Wang and Witten, 1997). During feature selection the attribute with the smallest standardized coefficient is iteratively removed until no 
improvement is observed in the error estimation. The error estimation is given by the Akaike Information Criterion (AIC) (Akaike, 1974).

(ii) Two decision trees based on the M5' algorithm (Wang and Witten, 1997) utilizing model (M5p) and regression (M5p-R) trees, were built. In tree structures, leaves represent classes and branches represent conjunctions of features that lead to these classes. The m5p model tree is using a linear regression function on each leaf, and the $\mathrm{m} 5 \mathrm{pR}$ regression tree, is using a constant value on each leaf node, i.e. the average value of the instances reaching at that leaf, instead (Quinlan, 1992; Wang and Witten, 1997).

(iii) Two meta-learning algorithms, the additive regression (Friedman, 2002) and bagging (Breiman, 1996) algorithms, each using two different regression trees as base classifiers (m5pR and REPTrees) (Kaariainen and Malinen, 2004; Quinlan, 1992; Wang and Witten, 1997) were used. In additive regression algorithm, during the training procedure, in each iteration, a regression tree is built using the residuals of the previous tree as training data. In the two cases of additive regression meta-classification, the shrinkage parameter, $v$, indicating the learning rate, was set equal to 0.5 and the number of the regression trees, $r$-num, was set equal to ten. These values were selected after grid-search experiments $(v=\{0.1,0.3,0.5,0.7,0.9\}, r t$-num $=\{5,10,15,20\})$ on a randomly selected subset of the training data, of size approximately equal to $20 \%$ of the size of the training set. In the bagging algorithm, the dataset is split in multi subsets utilizing one regression tree for each one of them and the final prediction value is the average of the values predicted by each regression tree. In these two cases, the number of the regression trees (rt-num) was set equal to ten after a number of grid-search experiments $(r t-n u m=\{5,10,15$, $20\}$ ) on the randomly selected subset of the training data, representing the $20 \%$ of the size of the full training dataset.

\subsubsection{Databases and feature set}

In the evaluation experiments two databases were used: the American-English speech database CSTR US KED TIMIT (CSTR, 2001) and the Modern Greek speech prosodic database, WCL-1 (Zervas et al., 2008). KED TIMIT consists of 453 
phonetically balanced sentences (3400 words approximately) uttered by a Native American male speaker. The WCL-1 prosodic database consists of 5500 words distributed in 500 paragraphs, each one of which may be a single word, a short sentence, a long sentence, or a sequence of sentences uttered by a female professional radio actress. The final corpus includes 390 declarative sentences, 44 exclamation sentences, 36 decision questions and 24 "wh" questions.

For the experiments on the KED TIMIT database, the phone set provided with the database (CSTR, 2001) which consists of 44 phones was adopted. For the experiments using the WCL-1 database the phone set provided with the database (Zervas et al., 2008) consisting of 34 phones was adopted. In all experiments, the manually labelled phone durations were used as the ground truth (reference) durations.

In this work, a number of features, which have been reported successful in the literature (Campbell, 1992; Crystal and House, 1988; Klatt, 1987; Goubanova and King, 2008; Riley, 1992; van Santen, 1994), are considered for the task of phone duration modelling. From each utterance 33 features were computed, and for some of them their temporal neighbours were also used, defined on the level of the respective feature, i.e. phone-level, syllable-level, word-level. These features are summarized in the following:

(i) eight phonetic features: the phone class (consonants/non-consonants), the phone types (vowels, diphthongs, schwa, consonants), the vowel height (high, middle or low), the vowel frontness (front, central or back), the lip rounding (rounded/unrounded), the manner of production (plosive, fricative, affricate, approximant, lateral, nasal), the place of articulation (labial, labio-dental, dental, alveolar, palatal, velar, glottal), the consonant voicing. Along with the aforementioned features, which concern each current instance, the two previous and the two next instances (temporal context information) were also used,

(ii) three segment-level features: the phone name with the temporal context information of the neighbouring instances (previous, next), the position of the phone in the syllable and the onset-coda type (if the specific phone is before or after the vowel in the syllable).

(iii) thirteen syllable-level features: the position type of the syllable in the word (single, initial, middle or final) with the temporal context information of the neighbouring instances (previous, next), the number of all the syllables in the word, the number of the accented syllables and the number of the stressed syllables since the 
last and to the next phrase break (i.e. the break index tier of ToBI with values, $0,1,2$, 3, 4,) (cf. below, item (v)) (Silverman et al., 1992), syllable's onset-coda size (the number of phones before and after the vowel of the syllable) with the temporal context information of previous and next instances, the onset-coda type (if the consonant before and after the vowel in the syllable is voiced or unvoiced) with the temporal context information of previous and next instances, the position of the syllable in the word and the onset-coda consonant type (the manner of production of the consonant before and after the vowel in the syllable).

(iv) two word-level features: the part-of-speech (noun, verb, adjective, etc) and the number of syllables of the word.

(v) one phrase-level feature: the syllable break (i.e. the phrase break after the syllable) with the temporal context information of the neighbouring (two previous, two next) instances. The syllable break feature is implemented based on the break index tier of ToBI $(0,1,2,3,4)$ (Silverman et al., 1992). The break index specifies an inventory of numbers expressing the strength of a prosodic juncture. The prosodic association of words is shown using the break index tier, by labelling the end of each word for the subjective strength of its association with the next word on a scale from 0 (strongest perceived conjoining) to 4 (most disjoint), defined as follows (Beckman and Ayers, 1994; Silverman et al., 1992; Huang et al., 2001):

a. 0 for cases of clear phonetic marks of clitic groups,

b. 1 most phrase-medial word boundaries,

c. 2 a strong disjuncture marked by a pause or virtual pause, but with no tonal marks; i.e. a well-formed tune continues across the juncture or a disjuncture that is weaker than expected at what is tonally a clear intermediate or full intonation phrase boundary,

d. 3 intermediate intonation phrase boundary; i.e. marked by a single phrase tone affecting the region from the last pitch accent to the boundary,

e. 4 full intonation phrase boundary; i.e. marked by a final boundary tone after the last phrase tone.

(vi) six accentual features: the ToBI accents and boundary tones with the temporal context information of the neighbouring (previous, next) instances, the last-next accent (the number of the syllables since the last and to the next accented syllable) and we also included the stressed-unstressed syllable feature (if the syllable is stressed or not) and the accented-unaccented syllable feature (if the syllable is accented or not) 
with the temporal context information of the neighbouring (two previous, two next) instances.

The overall size of the feature vector, which was used for the individual phone duration models, including the aforementioned features and their temporal context information as reported above (one or two previous and next instances on the level of the respective feature, phone-level, syllable-level, word-level) is 93.

In all experiments an experimental protocol based on 10-fold cross-validation was followed. Specifically, in each fold the training data were split in two portions, the training dataset and the development dataset. The former, amounting to approximately $60 \%$ of the full dataset, was used for the training of the individual phone duration predictors, and the latter, amounting to approximately $30 \%$ of the full dataset, for the training of the fusion algorithm and the two-stage scheme with the implementation of the feature vector extension stage which are described next in Section 4.2 and Section 4.3 respectively. Furthermore, the test dataset, amounting to approximately $10 \%$ of the full dataset, was used for evaluating the performance of the eight individual duration prediction algorithms, as well as the performance of the fusion scheme the two-stage scheme (described next in Section 4.2 and Section 4.3 respectively).

\subsubsection{Performance evaluation metrics}

The experimental results were evaluated using the two most commonly used figures of merit, namely the mean absolute error (MAE) and the root mean squared error (RMSE), between the predicted duration and the actual (reference) duration of each phone (Chen et al., 1998; Goubanova and King, 2008; van Santen, 1992; Yamagishi et al., 2008). Due to the squaring of values in the RMSE, large errors (outliers) are weighted heavily, which makes this figure of metric more sensitive to outliers than the MAE (Witten and Frank, 2005). This sensitivity of the RMSE makes it a more illustrative measurement concerning the outliers, e.g. the gross errors, in comparison to the MAE. These two figures of metrics are formulated as:

$$
R M S E=\sqrt{\frac{\sum_{i=1}^{N}\left(F\left(x_{i}\right)-y_{i}\right)^{2}}{N}}
$$

and 


$$
M A E=\frac{\sum_{i=1}^{N}\left|F\left(x_{i}\right)-y_{i}\right|}{N}
$$

where $N$ is the number of the test instances, $y_{i}$ is the actual duration of $i t h$ instance and $F\left(x_{i}\right)$ is the predicted value for the $i t h$ instance.

\subsubsection{Experimental results}

In this section, the performance of the eight individual algorithms on both databases using the entire feature set described in Section 4.1.2.2, is examined. The RMSE, the MAE and the standard deviation of the absolute error (STD of AE) for all individual algorithms specified earlier are shown in Table 4.1, where Table 4.1 (a) presents the results obtained on KED TIMIT and Table 4.1 (b) the ones on the WCL-1 database. The results of the best performing model, among the eight individual prediction models, are in bold. As can be seen, on both databases, the proposed support vector regression (SMOreg) model, implemented with the SMO regression algorithm, outperforms all the other models.

Table 4.1 (a). Mean Absolute Error (MAE), standard deviation of absolute error (STD of AE) and Root Mean Square Error (RMSE) in milliseconds, for the eight individual phone duration prediction algorithms on the KED TIMIT database.

\begin{tabular}{lccc}
\hline Individual models (KED TIMIT database) & MAE $(\boldsymbol{m s})$ & STD of $\boldsymbol{A E}(\boldsymbol{m s})$ & $\boldsymbol{R} M \boldsymbol{S E}(\boldsymbol{m s})$ \\
\hline SMOreg & $\mathbf{1 4 . 9 5}$ & $\mathbf{1 4 . 1 1}$ & $\mathbf{2 0 . 5 6}$ \\
Add. Reg. m5pR & 15.82 & 14.34 & 21.35 \\
Add. Reg. REPTrees & 16.29 & 15.06 & 22.19 \\
Bagging m5pR & 16.51 & 14.76 & 22.14 \\
m5p & 16.62 & 14.77 & 22.23 \\
Bagging REPTrees & 16.69 & 15.89 & 23.04 \\
m5pR & 16.93 & 15.16 & 22.72 \\
Linear Regression & 17.15 & 15.16 & 22.89 \\
\hline
\end{tabular}


Table 4.1 (b). Mean Absolute Error (MAE), standard deviation of absolute error (STD of AE) and Root Mean Square Error (RMSE) in milliseconds, for the eight individual phone duration prediction algorithms on the WCL-1 database.

\begin{tabular}{lccc}
\hline Individual models (WCL-1 database) & MAE (ms) & STD of $\boldsymbol{A E}(\boldsymbol{m s})$ & $\boldsymbol{R M S E}(\boldsymbol{m s})$ \\
\hline SMOreg & $\mathbf{1 6 . 7 8}$ & $\mathbf{1 8 . 8 1}$ & $\mathbf{2 5 . 2 1}$ \\
Linear Regression & 18.00 & 19.02 & 26.19 \\
Add. Reg. REPTrees & 18.08 & 19.97 & 26.94 \\
Add. Reg. m5pR & 18.13 & 19.16 & 26.38 \\
Bagging m5pR & 18.14 & 19.63 & 26.72 \\
m5p & 18.31 & 20.08 & 27.17 \\
Bagging REPTrees & 18.93 & 20.32 & 27.77 \\
m5pR & 19.07 & 20.10 & 27.71 \\
\hline
\end{tabular}

For more convenience for the reader, in Fig 4.1 the results for the SMOreg and the second-best model are presented. Specifically, on the KED TIMIT database (Fig. 4.1(a)) the SMOreg model outperformed the second-best model, i.e. the metaclassifier additive regression using $\mathrm{m} 5 \mathrm{pR}$ ( $A d d$. Reg. $m 5 p R$ ) model, by approximately $5.5 \%$ and $3.7 \%$ in terms of MAE and RMSE respectively. On the WCL-1 database (Fig. 4.1(b)) the SMOreg model outperformed the second-best model, i.e. the Linear Regression model, by approximately $6.8 \%$ and $3.7 \%$ in terms of MAE and RMSE respectively. This advantage of the SMOreg models, on both databases, is owed to the advantage of SVMs to cope better with high-dimensional feature spaces (Vapnik, 1995; Vapnik, 1998), when compared to the other classification and regression algorithms. 


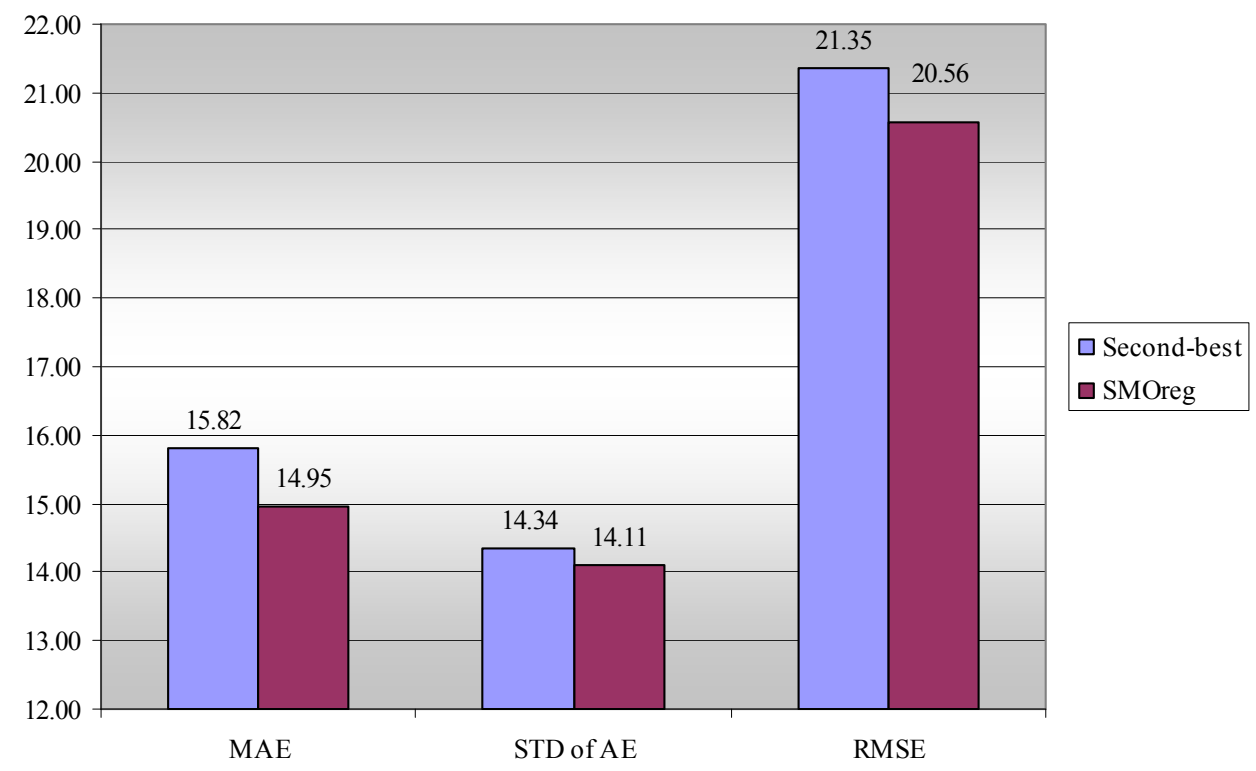

(a) results on the KED TIMIT database

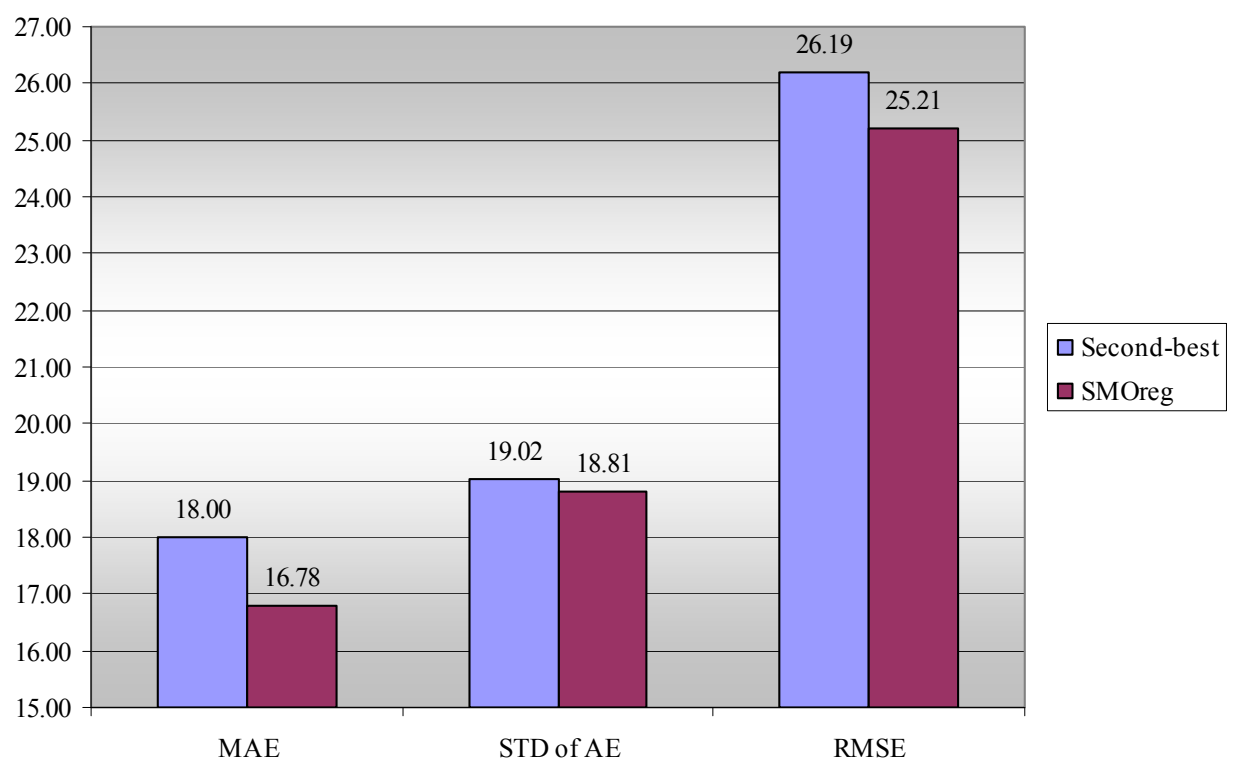

(b) results on the WCL-1 database

Fig. 4.1. Mean Absolute Error (MAE), standard deviation of absolute error (STD of AE) and Root Mean Square Error (RMSE) in milliseconds, for individual SMOreg and the second-best phone duration prediction algorithms on: (a) the KED TIMIT database, and (b) the WCL-1 database.

In the present work, clustering of the instances on the basis of (i) vowels/consonants categorization, (ii) phonetic categories and (iii) individual phones is considered. This offers different degree of detail and allows us to gain insights 
about the advantages and disadvantages of each algorithm. In Table 4.2 the performance per phonetic category as well as for the vowel/consonant categorization of the eight individual phone duration models, implemented by different algorithms, on the KED TIMIT (Table 4.2 (a)) and the WCL-1 (Table 4.2 (b)) databases is presented. As can be seen, the SMOreg model demonstrates the lowest RMSE on both databases, in all cases except for the Affricates on KED TIMIT, where the lowest RMSE is observed for the REPTrees and the SMOreg achieves the second-best performance.

Table 4.2. Root Mean Square Error in milliseconds, per phonetic category for the eight individual phone duration prediction algorithms on: (a) the KED TIMIT database, and (b) the WCL-1 database.

(a) results on the KED TIMIT database

\begin{tabular}{|c|c|c|c|c|c|c|c|c|}
\hline \multirow{2}{*}{$\begin{array}{l}\text { KED TIMIT } \\
\text { database }\end{array}$} & \multirow{2}{*}{$L R$} & \multirow{2}{*}{$m 5 p$} & \multirow{2}{*}{$m 5 p R$} & \multicolumn{2}{|c|}{ Additive Regression } & \multicolumn{2}{|c|}{ Bagging } & \multirow{2}{*}{ SMOreg } \\
\hline & & & & $m 5 p R$ & REPTrees & $m 5 p R$ & REPTrees & \\
\hline Vowel & 24.56 & 24.18 & 25.46 & 23.67 & 24.87 & 24.78 & 26.34 & 22.72 \\
\hline Consonant & 21.72 & 20.86 & 20.74 & 19.69 & 20.24 & 20.24 & 20.60 & 19.02 \\
\hline \multirow{2}{*}{ Phonetic category } & \multirow{2}{*}{$L R$} & \multirow{2}{*}{$m 5 p$} & \multirow{2}{*}{$m 5 p R$} & \multicolumn{2}{|c|}{ Additive Regression } & \multicolumn{2}{|c|}{ Bagging } & \\
\hline & & & & $m 5 p R$ & REPTrees & $m 5 p R$ & REPTrees & DMU \\
\hline Vowel & 24.56 & 24.18 & 25.46 & 23.67 & 24.87 & 24.78 & 26.34 & 22.72 \\
\hline Affricate & 22.44 & 24.41 & 23.48 & 22.86 & 21.72 & 22.96 & 23.34 & 21.88 \\
\hline Approximant & 22.23 & 22.44 & 23.09 & 21.77 & 22.56 & 22.59 & 24.07 & 20.42 \\
\hline Fricative & 22.51 & 21.67 & 21.10 & 20.19 & 20.69 & 20.63 & 20.96 & 19.63 \\
\hline Lateral & 21.16 & 20.98 & 21.18 & 20.29 & 21.16 & 20.52 & 21.89 & 19.77 \\
\hline Nasal & 18.59 & 17.88 & 17.80 & 17.11 & 16.94 & 17.28 & 17.57 & 16.53 \\
\hline Plosive & 23.39 & 22.07 & 21.62 & 20.26 & 20.97 & 21.04 & 20.80 & 19.61 \\
\hline
\end{tabular}

(b) results on the WCL-1 database

\begin{tabular}{|c|c|c|c|c|c|c|c|c|}
\hline \multirow{2}{*}{ WCL-1 database } & \multirow{2}{*}{$L R$} & \multirow{2}{*}{$m 5 p$} & \multirow{2}{*}{$m 5 p R$} & \multicolumn{2}{|c|}{ Additive Regression } & \multicolumn{2}{|c|}{ Bagging } & \multirow{2}{*}{ SMOreg } \\
\hline & & & & $m 5 p R$ & REPTrees & $m 5 p R$ & REPTrees & \\
\hline Vowel & 24.22 & 24.68 & 26.04 & 24.51 & 25.18 & 24.91 & 26.62 & 23.12 \\
\hline Consonant & 27.86 & 29.25 & 29.13 & 27.97 & 28.44 & 28.27 & 28.77 & 26.57 \\
\hline \multirow{2}{*}{ Phonetic category } & \multirow{2}{*}{$L R$} & \multirow{2}{*}{$m 5 p$} & \multirow{2}{*}{$m 5 p R$} & \multicolumn{2}{|c|}{ Additive Regression } & \multicolumn{2}{|c|}{ Bagging } & SMOreo \\
\hline & & & & $m 5 p R$ & REPTrees & $m 5 p R$ & REPTrees & Ninuteg \\
\hline Vowel & 24.22 & 24.68 & 26.04 & 24.51 & 25.18 & 24.91 & 26.62 & 23.12 \\
\hline Affricate & 24.72 & 27.61 & 24.62 & 22.22 & 20.74 & 25.83 & 23.57 & 20.73 \\
\hline Fricative & 25.67 & 27.04 & 26.93 & 25.79 & 26.23 & 25.57 & 26.45 & 23.95 \\
\hline Liquid & 19.46 & 19.19 & 19.55 & 18.84 & 17.83 & 18.47 & 18.02 & 16.38 \\
\hline Nasal & 22.44 & 22.94 & 23.11 & 22.27 & 22.15 & 22.18 & 22.27 & 20.62 \\
\hline Plosive & 34.22 & 36.33 & 36.03 & 34.62 & 35.64 & 35.48 & 36.14 & 33.69 \\
\hline
\end{tabular}

In Table 4.3 the phone duration prediction results obtained on the level of individual phones are presented. Specifically, Table 4.3 (a) shows the RMSE for the 
44 phone set of the KED TIMIT database and Table 4.3 (b) for the 34 phone set of the WCL-1 database. The results for the best performing algorithm are in bold. As shown in the tables, despite the fact that the SMOreg model demonstrates the highest overall performance on both databases (refer to Table 4.1), in one phonetic category (Affricates in Table 4.2 (a)) and in some particular phones, such as ch, ay, etc (Table 4.3), other models offer a higher phone duration prediction accuracy. For instance, on the KED TIMIT database, the highest accuracy for the phone ch is observed for the Linear Regression model, while for the phone ay the highest accuracy is for the $m 5 p$ model (refer to Table 4.3 (a)). These specific results, and other similar cases shown in the Table 4.3, are in support of our observation that different algorithms perform better in different phonetic categories and phones. This indicates that an appropriate fusion of the outputs of the individual phone duration prediction models could be beneficial for reducing the overall error rate. This fusion scheme is presented and evaluated in the next section (Section 4.2) 
Table 4.3 (a). Root Mean Square Error in milliseconds, per phone for the eight individual phone duration prediction algorithms on the KED TIMIT database

\begin{tabular}{|c|c|c|c|c|c|c|c|c|c|}
\hline \multirow{2}{*}{$\begin{array}{c}\text { KED TIMIT } \\
\text { database }\end{array}$} & \multirow{2}{*}{$\begin{array}{c}I P A \\
\text { Symbols }\end{array}$} & \multirow{2}{*}{$L R$} & \multirow{2}{*}{$m 5 p$} & \multirow{2}{*}{$m 5 p R$} & \multicolumn{2}{|c|}{ Additive Regression } & \multicolumn{2}{|c|}{ Bagging } & \multirow{2}{*}{${ }_{s}^{S M O r e g}$} \\
\hline & & & & & $m 5 p R$ & REPTrees & $m 5 p R$ & REPTrees & \\
\hline$a a$ & $b$ & 27.81 & 25.57 & 28.01 & 24.57 & 27.27 & 26.71 & 29.22 & 25.64 \\
\hline ae & $\mathfrak{x}$ & 31.40 & 30.97 & 31.67 & 29.75 & 31.19 & 31.64 & 33.13 & 29.23 \\
\hline$a h$ & $\wedge$ & 19.67 & 22.34 & 22.27 & 20.31 & 21.50 & 20.88 & 22.27 & 19.38 \\
\hline ao & ग: & 32.79 & 29.21 & 32.95 & 30.54 & 32.11 & 32.66 & 33.29 & 29.65 \\
\hline$a w$ & $a v$ & 33.46 & 32.89 & 37.35 & 34.55 & 38.49 & 37.25 & 40.02 & 33.07 \\
\hline$a x$ & ə & 16.10 & 15.66 & 16.06 & 15.16 & 15.56 & 15.54 & 15.93 & 14.80 \\
\hline ay & aI & 37.12 & 32.78 & 38.37 & 34.43 & 34.04 & 36.64 & 37.23 & 34.51 \\
\hline$b$ & $\mathrm{~b}$ & 23.89 & 22.36 & 23.42 & 22.24 & 23.03 & 22.52 & 21.19 & 21.33 \\
\hline$c h$ & ts & 19.69 & 23.34 & 21.17 & 20.48 & 20.43 & 19.89 & 22.36 & 20.57 \\
\hline$d$ & d & 20.77 & 19.36 & 19.66 & 19.12 & 20.05 & 9.32 & 20.54 & 18.26 \\
\hline$d h$ & б & 17.56 & 16.03 & & 15.19 & .57 & .16 & 15.30 & 5.14 \\
\hline$d x$ & $\mathrm{~d} / \mathrm{t}$ & 11.08 & 10.38 & 11.30 & 9.99 & 8.78 & 9.54 & 8.86 & 9.63 \\
\hline eh & $\varepsilon$ & 20.94 & 20.39 & 22.50 & 21.41 & 21.44 & 21.32 & 22.61 & 19.05 \\
\hline el & əl & 21.39 & 27.24 & 21.52 & 20.79 & 18.98 & 19.97 & 21.05 & 22.32 \\
\hline em & əm & 13.61 & 15.31 & & & 13 & .28 & 13.99 & 1.58 \\
\hline en & ən & 22.26 & 24.60 & 25.01 & 23.18 & .67 & 22.44 & 21.80 & 21.01 \\
\hline$e r$ & $3 r$ & 28.41 & 29.28 & 28.73 & 27.09 & 27.77 & 28.15 & 29.87 & 25.29 \\
\hline ey & eI & 27.76 & 26.99 & 29.43 & 28.12 & 29.90 & 28.72 & 31.36 & 26.73 \\
\hline$f$ & $\mathrm{f}$ & 22.84 & 23.90 & 21.52 & 20.09 & 21.05 & 21.08 & 22.43 & 18.91 \\
\hline$g$ & $\mathrm{~g}$ & 18.23 & 17.14 & 18.73 & 17.04 & 17.65 & 17.88 & 17.62 & 16.22 \\
\hline$h h$ & $\mathrm{~h}$ & 19.13 & 18.79 & 18.82 & 18.52 & 18.73 & 18.28 & 18.73 & 17.54 \\
\hline$i h$ & I & 19.38 & 19.76 & 20.16 & 19.09 & 19.81 & 19.82 & 20.86 & 17.53 \\
\hline iy & i: & 23.04 & 23.06 & 23.87 & 22.05 & 24.93 & 23.27 & 25.39 & 20.99 \\
\hline$j h$ & d3 & 24.36 & 25.22 & 25.14 & 24.56 & 22.68 & 25.08 & 24.07 & 22.85 \\
\hline$k$ & $\mathrm{k}$ & 22.18 & 21.82 & 20.62 & 18 & 63 & 94 & 18.93 & 7.64 \\
\hline$l$ & 1 & & & & & & & & \\
\hline$m$ & $\mathrm{~m}$ & 16 & 15.32 & 16 & 15 & & 31 & 17 & 14.38 \\
\hline$n$ & $\mathrm{n}$ & 18. & 17.65 & 17. & 16.70 & 18 & 16.80 & 16.32 & 16.19 \\
\hline$n g$ & $\eta$ & 22.38 & 20.86 & 20.13 & 19.90 & 20.88 & 20.61 & 22.41 & 20.91 \\
\hline ow & Ov & 28.12 & 28.98 & 28.93 & 27.20 & 28.85 & 27.73 & 30.68 & 25.54 \\
\hline oy & כI & 25.45 & 30.16 & 34.58 & 28.81 & 30.61 & 33.13 & 34.72 & 31.19 \\
\hline$p$ & $\mathrm{p}$ & 25.06 & 24.90 & 22.50 & 21.05 & 21.32 & 21.94 & 21.25 & 20.45 \\
\hline$r$ & $\lambda / \mathrm{r}$ & 19.20 & 18.84 & 20.18 & 19.28 & 20.11 & 19.92 & 21.18 & 18.25 \\
\hline$s$ & $\mathrm{~s}$ & 26.37 & 24.47 & 24.31 & 23.46 & 24.45 & 24.36 & 24.54 & 23.21 \\
\hline$s h$ & $\int$ & 19.71 & 21.72 & 19.28 & 18.30 & 20.53 & 18.49 & 20.27 & 16.41 \\
\hline$t$ & $\mathrm{t}$ & & 25.60 & & & & 24.72 & 24.93 & 23.37 \\
\hline th & $\theta$ & 24.09 & 26.39 & 29.14 & 25.58 & 21.31 & 25.21 & 22.59 & 22.05 \\
\hline$u h$ & U & 20.64 & 20.61 & 23.10 & 20.45 & 25.35 & 22.68 & 26.16 & 19.88 \\
\hline$u w$ & u: & 27. & 27.73 & 29.05 & 28 & 30.35 & 29.40 & 33.64 & 24.97 \\
\hline$v$ & $\mathrm{v}$ & 17.26 & 17.31 & 16.72 & 16.93 & 17.15 & 16.66 & 17.34 & 16.26 \\
\hline$w$ & w & 20.28 & 20.09 & 22.35 & 19.81 & 20.93 & 20.89 & 22.59 & 19.12 \\
\hline$y$ & $\mathrm{j}$ & & 19.08 & & 18.80 & 19.42 & 19.22 & 20.56 & 16.34 \\
\hline$z$ & $\mathrm{z}$ & 22.38 & 20.42 & 19.94 & 19.07 & 19.37 & 19.24 & 19.10 & 18.99 \\
\hline$z h$ & 3 & 25.60 & 28.40 & 25.25 & 22.62 & 26.38 & 23.95 & 27.28 & 24.66 \\
\hline
\end{tabular}


Table 4.3 (b). Root Mean Square Error in milliseconds, per phone for the eight individual phone duration prediction algorithms on the WCL-1 database

\begin{tabular}{|c|c|c|c|c|c|c|c|c|c|}
\hline \multirow{2}{*}{$\begin{array}{c}\text { WCL-1 } \\
\text { database }\end{array}$} & \multirow{2}{*}{$\begin{array}{c}I P A \\
\text { Symbols }\end{array}$} & \multirow{2}{*}{$L R$} & \multirow{2}{*}{$m 5 p$} & \multirow{2}{*}{$m 5 p R$} & \multicolumn{2}{|c|}{ Additive Regression } & \multicolumn{2}{|c|}{ Bagging } & \multirow{2}{*}{ SMOreg } \\
\hline & & & & & $m 5 p R$ & REPTrees & $m 5 p R$ & REPTrees & \\
\hline$a$ & $\mathrm{a}$ & 24.25 & 25.85 & 25.76 & 24.07 & 24.57 & 24.83 & 26.07 & 22.71 \\
\hline$b$ & $\mathrm{~b}$ & 21.05 & 24.66 & 24.41 & 21.84 & 22.05 & 22.33 & 22.53 & 20.20 \\
\hline$c$ & $t 5$ & 24.16 & 28.40 & 25.43 & 22.48 & 20.29 & 26.62 & 23.62 & 20.85 \\
\hline$D$ & ð & 22.64 & 23.08 & 25.08 & 24.24 & 24.39 & 24.00 & 26.25 & 22.64 \\
\hline$d$ & d & 19.33 & 20.40 & 23.54 & 21.01 & 21.44 & 21.39 & 24.61 & 20.10 \\
\hline$e$ & e & 25.05 & 25.11 & 26.69 & 25.62 & 26.79 & 25.71 & 26.48 & 24.05 \\
\hline$f$ & $\mathrm{f}$ & 30.13 & 34.11 & 30.41 & 29.95 & 33.13 & 29.50 & 31.94 & 29.56 \\
\hline$G$ & f & 30.72 & 37.75 & 37.14 & 31.68 & 31.56 & 33.82 & 33.99 & 29.89 \\
\hline$g$ & g & 34.43 & 38.79 & 34.94 & 35.14 & 40.05 & 34.30 & 37.80 & 33.85 \\
\hline$h$ & $\eta$ & 24.73 & 25.91 & 26.39 & 24.69 & 24.87 & 23.78 & 25.88 & 23.50 \\
\hline$i$ & $\mathrm{i}$ & 24.17 & 24.30 & 25.54 & 24.27 & 24.68 & 24.68 & 26.98 & 23.09 \\
\hline$j$ & d3 & 25.65 & 26.18 & 23.13 & 21.75 & 20.52 & 24.39 & 23.48 & 21.50 \\
\hline$K$ & $\mathrm{c}$ & 45.75 & 44.50 & 45.47 & 43.94 & 46.86 & 45.73 & 45.82 & 43.28 \\
\hline$k$ & $\mathrm{k}$ & 42.27 & 46.15 & 44.65 & 43.31 & 44.58 & 43.90 & 47.61 & 43.61 \\
\hline$k s$ & ks & 22.50 & 24.00 & 42.80 & 39.97 & 26.32 & 42.34 & 27.10 & 23.16 \\
\hline$L$ & $\Lambda$ & 24.98 & 32.86 & 32.93 & 29.20 & 28.64 & 29.98 & 29.43 & 26.64 \\
\hline$l$ & 1 & 19.95 & 19.34 & 20.63 & 19.85 & 20.17 & 19.65 & 20.96 & 18.31 \\
\hline$m$ & $\mathrm{~m}$ & 22.90 & 22.82 & 23.74 & 22.56 & 23.46 & 22.67 & 23.96 & 22.27 \\
\hline$N$ & $\mathrm{n}$ & 26.99 & 33.30 & 36.39 & 33.22 & 24.13 & 34.11 & 24.37 & 21.26 \\
\hline$n$ & $\mathrm{n}$ & 21.76 & 22.14 & 21.58 & 21.21 & 21.07 & 20.96 & 20.88 & 19.38 \\
\hline$o$ & o & 23.70 & 23.81 & 25.99 & 24.18 & 25.08 & 24.36 & 25.85 & 22.72 \\
\hline$p$ & $\mathrm{p}$ & 29.65 & 32.71 & 31.57 & 28.65 & 30.51 & 29.80 & 30.04 & 28.24 \\
\hline$Q$ & $\theta$ & 23.08 & 25.82 & 25.22 & 23.49 & 24.99 & 23.82 & 26.83 & 23.85 \\
\hline$r$ & $r$ & 18.64 & 17.53 & 17.30 & 17.06 & 14.94 & 16.41 & 14.53 & 13.85 \\
\hline$S$ & $\mathrm{~S}$ & 26.93 & 27.65 & 27.28 & 26.10 & 24.75 & 25.47 & 25.11 & 23.49 \\
\hline$t$ & $\mathrm{t}$ & 34.70 & 36.84 & 34.98 & 34.31 & 36.09 & 34.96 & 36.04 & 34.07 \\
\hline$u$ & $\mathrm{u}$ & 23.24 & 22.63 & 27.51 & 24.78 & 25.11 & 25.37 & 29.45 & 23.12 \\
\hline$v$ & $\mathrm{~V}$ & 23.87 & 24.11 & 26.09 & 25.80 & 34.70 & 25.56 & 27.08 & 24.86 \\
\hline$w$ & ps & 20.83 & 25.71 & 40.93 & 42.47 & 25.92 & 42.98 & 29.62 & 23.66 \\
\hline$X$ & ç & 22.75 & 24.38 & 26.33 & 24.45 & 23.44 & 25.03 & 25.95 & 21.44 \\
\hline$x$ & $\mathrm{x}$ & 20.33 & 24.82 & 26.45 & 23.35 & 21.87 & 24.98 & 25.06 & 21.58 \\
\hline$Y$ & $j$ & 26.68 & 28.56 & 29.65 & 28.08 & 27.41 & 28.37 & 28.39 & 26.82 \\
\hline$y$ & $\gamma$ & 20.77 & 20.35 & 22.98 & 21.03 & 21.35 & 21.38 & 21.64 & 19.68 \\
\hline$z$ & $\mathrm{z}$ & 23.05 & 22.38 & 23.31 & 22.64 & 23.13 & 22.98 & 24.68 & 21.58 \\
\hline
\end{tabular}

\subsubsection{Conclusions}

Here, the accuracy of various machine learning algorithms on the task of phone duration modelling was studied and the support vector regression algorithm was 
proposed on the task of phone duration modelling. The experimental results showed that on this task, Support Vector Machines (SVM), as a regression (SVR) model (SMOreg), outperforms various other machine learning techniques. Specifically, in terms of relative decrease of the mean absolute error and root mean square error, the SMO regression model outperformed the second-best model by approximately $5.5 \%$ and $3.7 \%$ on KED TIMIT, and by approximately $6.8 \%$ and $3.7 \%$ on the WCL-1 database, respectively.

Furthermore clustering the instances on the basis of (i) vowels/consonants categorization, (ii) phonetic categories and (iii) individual phones, offers a different degree of detail and allows to gain insights about the advantages and disadvantages of each algorithm. Inspired by the observation that different algorithms performed better in different phonetic categories or individual phones, a fusion scheme of the predictions of individual models is proposed in the next section.

\subsection{Fusion of multiple predictions using support vector regression}

In this section, a scheme for the fusion of different phone duration models, operating in parallel, is proposed. Specifically, the predictions from a group of dissimilar and independent to each other individual duration models are fed to a machine learning algorithm, which reconciles and fuses the outputs of the individual models, yielding more precise phone duration predictions. The performance of the proposed fusion scheme is evaluated on the American-English KED TIMIT and on the Greek WCL-1 databases. At the fusion stage, we evaluate the performance of twelve fusion techniques. The proposed fusion scheme, when implemented with SVRbased fusion, contributes to the improvement of the phone duration prediction accuracy over the one of the best individual model, by $1.9 \%$ and $2.0 \%$ in terms of relative reduction of the MAE and RMSE on KED TIMIT, and by $2.6 \%$ and $1.8 \%$ on the WCL-1 database. 


\subsubsection{Introduction}

All previous studies on phone and syllable duration modelling are restricted to the use of a single linear or non-linear regression algorithm. The only exception to this trend is the work of Iwahashi and Sagisaka (2000), where a hierarchical structure for syllable duration prediction using the outputs of a phone duration model was used. However, this structure is restricted to the post-processing of a single duration prediction model, and no extension to a parallel regression fusion of the duration predictions of multiple models has been studied.

In this proposed scheme, aiming at improving the accuracy of the prediction of the segmental durations (here phone durations), we propose a fusion scheme based on the use of multiple dissimilar phone duration predictors which operate on a common input, and whose predictions are combined using a regression fusion method. The proposed scheme is based on the observation that predictors implemented with different machine learning algorithms perform differently in dissimilar conditions. Hence, an appropriate combination of their outputs is expected to result in a new set of more precise phone duration predictions. Thus, an appropriate fusion scheme that can learn how to combine the outputs of a number of individual predictors in a beneficial manner, will contribute to the reduction of the overall prediction error, when compared to the error of each individual predictor.

Based on this assumption and the study reported above (Section 4.1) concerning the accuracy of the individual phone duration models for duration prediction on different levels of granularity: vowels/consonants, phonetic category and individual phones, in this section we investigate various implementations of the proposed fusion scheme. In the proposed fusion scheme the durations predicted by the individual duration models are fed as inputs to a machine learning algorithm referred to as fusion model, which uses these predictions and produces the final phone duration prediction. For the purpose of fusion, we evaluate twelve different (linear and non-linear) regression fusion techniques, which are the linear regression, decision trees, support vector regression, neural networks, meta-learning and lazy-learning algorithms, and finally average linear combination and best-case fusion.

The present study was inspired by the work of Kominek and Black (2004), where a family of acoustic models, providing multiple estimates for each boundary point, was used for segmenting a speech database, creating synthetic speech of higher 
quality using a corpus-based unit selection TTS system. This approach was found more robust than a single estimate, since by taking consensus values large labelling errors are less prevalent in the synthesis catalogue, which improves the resulting synthetic speech. To the extent of our knowledge, a parallel regression fusion of individual models has not yet been studied on the phone duration prediction or on the syllable duration prediction tasks.

\subsubsection{Fusion of models using support vector regression}

Phone duration modelling, which mainly relies on regression algorithms, suffers from specific types of errors. The most commonly occurring type of error is the bias (systematic) error (Freedman et al., 2007). This error is a constant shift of the predicted phone durations from the real ones and can be estimated as the difference between the real and predicted mean durations. Other prediction errors that may occur in the phone duration modelling task are small miss-predictions and gross errors (outliers) (Freedman et al., 2007). Small miss-predictions in phone duration, i.e. less than 20 milliseconds, apart from the cases of short-duration phones such as schwas and flaps, do not significantly affect the quality of the synthetic speech signal. However, larger than $20 \mathrm{~ms}$ errors have been reported to degrade the quality of synthetic speech (Wang et al., 2004). Here, is assumed that an appropriate combination of the predictions of a number of dissimilar phone duration prediction models will improve the overall phone duration prediction accuracy. This is because different phone duration models will err in a dissimilar manner, and the fusion of their outputs, through a machine learning technique, would be able to learn and compensate some of these errors. Especially, is supposed that such a fusion scheme, apart from improving the overall accuracy of duration prediction, will be able to reduce the amount of gross errors.

In Fig. 4.2, the block diagram of the proposed fusion scheme, which relies on the combination of predictions that are produced by multiple dissimilar phone duration models, which operate on a common input, is presented. As the figure presents, the predictions of the individual models are introduced into the fusion stage, where a machine learning algorithm uses them for obtaining more precise phone duration 
predictions. The training and the operational phases of the proposed fusion scheme is discussed in the following subsections.

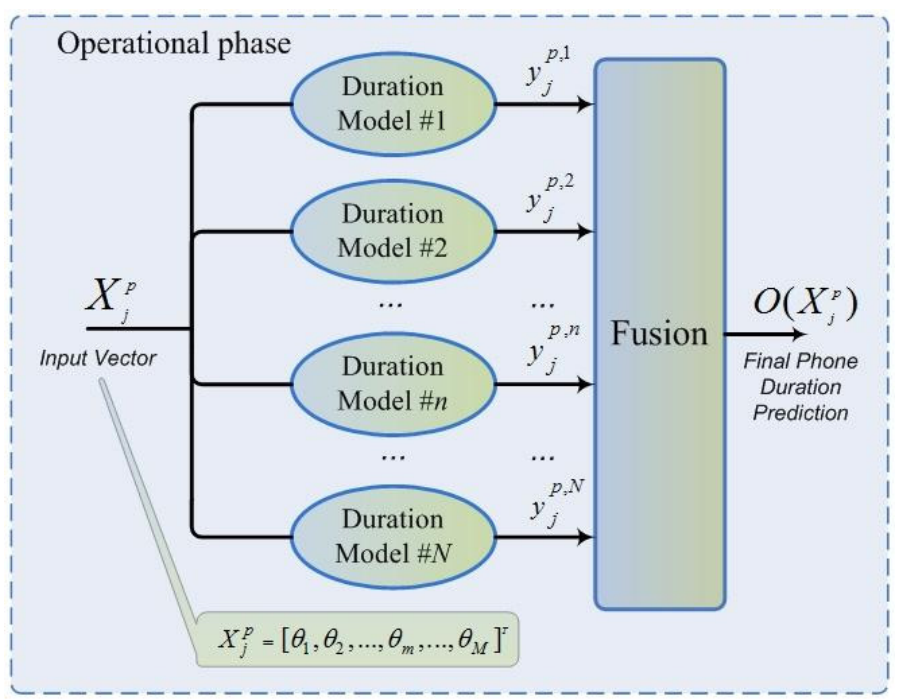

Fig. 4.2. Block diagram of the proposed fusion scheme, which exploits multiple dissimilar phone duration predictors, operating on a common input.

\subsubsection{Training of the fusion scheme}

The training of the proposed fusion scheme is an off-line two-step procedure, which relies on two non-overlapping datasets: the training and the development data. The training process can be summarized concisely as follows. During the first training step, the individual phone duration models are created using the training dataset. Subsequently, at the second step, these models are employed to process the development dataset. The outcome of this processing is a set of predictions, which together with the ground truth labels (manually annotated tags) serve as input for training the adjustable parameters of the fusion algorithm. This procedure can be formalized as follows:

Let us define a set of $N$ individual phone duration prediction models, $D M_{n}$, with $1 \leq n \leq N$. The input feature vector, $X_{j}^{p}$, for the $j t h$ instance $(1 \leq j \leq J)$ of the phone $p$, which is used for training the $N$ individual phone duration models, $D M_{n}$, is defined as:

$$
X_{j}^{p}=\left[\theta_{1}, \theta_{2}, \ldots, \theta_{m}, \ldots, \theta_{M}\right]^{T}, j=1,2, \ldots, J
$$


where $X \in \mathbb{C}^{M}$ ( $\mathbb{C}$ is the feature space) and $\theta_{m}$ is the $m t h$ feature $(1 \leq m \leq M)$ of the feature vector $X_{j}^{p}$.

Once the individual duration models are trained, they are fed with the development dataset. The outcome of its processing is the set of phone duration predictions, $y_{j}^{p, n}$, of the $n t h$ duration model for the $j t h$ instance of the phone $p$, to be predicted:

$$
y_{j}^{p, n}=f_{D M n}^{p}\left(X_{j}^{p}\right), \quad j=1,2, \ldots, J
$$

where $y_{j}^{p, n} \in \mathbb{R}^{N}$. The vector, $Y_{j}^{p}$, formed by appending the individual phone duration predictions, $y_{j}^{p, n}$, is

$$
Y_{j}^{p}=\left\{y_{j}^{p, n}\right\}^{T}, j=1,2, \ldots, J
$$

where $1 \leq n \leq N$, for the $j t h$ instance of the phone $p$, together with the ground truth labels, are used in the training of the fusion algorithm. Once the fusion stage is trained, the proposed composite phone duration modelling scheme, shown in Fig. 4.2, is ready for operation.

\subsubsection{Operation of the fusion scheme}

In the operational mode, the input vector, $X_{j}^{p}$, for the $j$ th instance of the phone $p$ of the test dataset, appears as input to the $N$ individual phone duration prediction models, $D M_{n}$, with $1 \leq n \leq N$ (refer to Fig. 4.2). Their outputs, $y_{j}^{p, n}$, as computed in eq. (4.9), form the vector of predictions $Y_{j}^{p}$, which serves as input for the fusion stage. At the fusion stage the vector $Y_{j}^{p}$ is processed by the fusion algorithm, which computes the final phone duration prediction for the $j t h$ instance as:

$$
O_{j}^{p}=g^{p}\left(Y_{j}^{p}\right), \quad j=1,2, \ldots, J
$$

with $O_{j}^{p} \in \mathbb{R}$.

The fusion of multiple different predictions is expected to contribute to the reduction of the types of errors described above (first paragraph in section 4.2.2), and consequently to contribute to the decrease of the overall error rate. This expectation is based on the observation that different predictors, which rely on different machine 
learning algorithms, err in a dissimilar manner. Employing an appropriate fusion scheme, which is capable to learn the proper mapping between a set of noisy predictions and the true phone duration values, could turn out beneficial in terms of improved accuracy.

\subsubsection{Experimental setup}

Based on the observation that predictors implemented with different machine learning algorithms perform differently in dissimilar conditions and on the study reported above (Section 4.1) concerning the accuracy of the individual phone duration models for duration prediction on different levels of granularity: vowels/consonants, phonetic category and individual phones, in this section we investigate various implementations of the proposed fusion scheme.

Here the practical usefulness of the proposed approach, using the individual phone duration models reported and evaluated earlier (Section 4.1), and implementing them in the fusion scheme described above, is investigated. Since the various individual phone duration models as well as the speech databases used in the experiments and the experimental protocol that is followed here are based on the study presented above in Section 4.1, are described again briefly in the next subsection for the convenience of the reader. Furthermore, the fusion algorithms involved in the phone duration prediction fusion scheme, are described in the following subsections.

\subsubsection{Individual phone duration models}

In the present work we consider the eight machine learning algorithms for phone duration modelling, evaluated earlier, the outputs of which are then fed to the fusion model. These algorithms are well known and have successfully been used over the years, in different modelling tasks. In brief, the eight individual phone duration modelling algorithms that we consider here are:

(i) the linear regression (LR) (Witten and Frank, 2005) using Akaike's Information Criterion (AIC) (Akaike, 1974) in backward stepwise selection 
(BSS) (Kohavi and John, 1997) procedure eliminating unnecessary variables of the training data,

(ii) the $\mathrm{m} 5 \mathrm{p}$ model tree, using a linear regression function on each leaf, and the m5pR regression tree, using a constant value on each leaf node instead (Quinlan, 1992; Wang and Witten, 1997).

(iii) two additive regression algorithms (Friedman, 2002) and two bagging algorithms (Breiman, 1996) were used, by using two different regression trees (m5pR and REPTrees) (Kaariainen and Malinen, 2004; Quinlan, 1992; Wang and Witten, 1997) as base classifiers in each case. In the two cases of additive regression meta-classification, the shrinkage parameter, $v$, indicating the learning rate, was set equal to 0.5 and the number of the regression trees, $r t$ num, was set equal to ten. These values were selected after grid-search experiments $(v=\{0.1,0.3,0.5,0.7,0.9\}, r t-n u m=\{5,10,15,20\})$ on a randomly selected subset of the training data, of size approximately equal to $20 \%$ of the size of the training set. In the two cases of the bagging algorithm, in a similar manner, the number of the regression trees (rt-num) was set equal to ten after a number of grid-search experiments $(r t-n u m=\{5,10,15,20\})$ on the randomly selected subset of the training data, representing the $20 \%$ of the size of the full training dataset.

(iv)Finally, the support vector regression (SVR) model (Platt, 1999), which employs the sequential minimal optimization (SMO) algorithm for training a support vector classifier (Smola and Scholkopf, 1998), was used. In our experiments the RBF kernel was used as mapping function. The parameters $\varepsilon$ and $C$, where $\varepsilon \geq 0$ is the maximum deviation allowed during training and $C>0$ is the penalty parameter for exceeding the allowed deviation, were set equal to $10^{-3}$ and $10^{-1}$ respectively. This was done after a grid search $\left(\varepsilon=\left\{10^{-1}\right.\right.$, $\left.\left.10^{-2}, \ldots, 10^{-5}\right\}, C=\{0.05,0.1,0.3,0.5,0.7,1.0,10,100\}\right)$ on the randomly selected subset of the training data, representing the $20 \%$ of the size of the full training dataset..

The motivation to select these algorithms was based on previous research (Iwahashi and Sagisaka, 2000; Lee and Oh, 1999; Riley, 1992; Takeda et al., 1989; Yamagishi et al., 2008), where these algorithms were reported successful on the segmental duration modelling task. Along with the phone duration prediction task, 
many of these algorithms have also been used in syllable duration prediction task, supporting different languages and databases.

\subsubsection{Fusion algorithms}

The outputs of the eight individual duration models, outlined in Section 4.1, serve as the input for the fusion stage, which combines and disambiguates their predictions (refer to Fig. 4.2). The fusion stage can be implemented through different linear or nonlinear machine learning techniques. In this work in order to select the most advantageous fusion method, twelve different algorithms for numerical prediction are evaluated. These include the eight algorithms outlined in Section 4.1, which in this case are used as fusion models, as well as (i) the radial basis function neural network (RBFNN) with Gaussian kernel (Park and Sandberg, 1993), (ii) the instance-based algorithm (IBK) (Aha and Kibler, 1991), which is a lazy-learning algorithm, (iii) the average linear combination, where the mean value of all individual prediction estimations of the first stage duration models is regarded as the final phone duration prediction value, and (iv) the best-case selection method, where for each instance the best prediction is selected among the predictions of all first-stage individual phone duration models. The selection of the best duration model per instance can be performed for different categories of clusters, such as voiced/unvoiced, vowels/consonants, phonetic category, individual phones, diphones and triphones. The last two methods, namely the average linear combination and the best case selection are well-known fusion schemes, and therefore in the present study they serve as intuitive reference points. In all the implementations listed above, the fusion method takes as input the predictions of the individual phone duration models, $y_{j}^{p, n}$, obtained at the first stage and combines them for obtaining the final prediction $O_{j}^{p}\left(X_{j}^{p}\right)$.

The functioning of the first eight machine learning techniques was already outlined in Section 4.1, therefore here we focus on the other four techniques. The RBFNN (see Section 3.2.3) implements a Gaussian radial basis function network, deriving the centres and widths of hidden units using $k$-means (Hartigan, 1975) and combining the outputs obtained from the hidden layer using logistic regression if the 
class is nominal, and linear regression if it is numeric. The activations of the basis functions are normalized to sum to unity before they are fed into the linear models. The number of clusters and the minimum standard deviation for the clusters are the free parameters of the algorithm (Witten and Frank, 2005). The number of clusters, num-cl, to be generated by the $k$-means algorithm and the minimum standard deviation, $c l$-std, for the clusters were set equal to 135 and $10^{-2}$, respectively. These parameters were determined by a grid search $($ num-cl $=\{5,10, \ldots, 200\}, c l-s t d=\{0.001$, $0.01,0.1,0.5\})$ on the randomly selected subset of the training dataset, representing the $20 \%$ of the size of the full training dataset.

The IBK (see Section 3.2.4) is an instance-based algorithm (Mitchell, 1997), which belongs to the lazy-learning algorithms. In the training phase, it stores the training instances verbatim, and in the prediction phase, it searches for the instance that most closely resembles the target instance in order to predict the target value. This is calculated through the use of a distance function. In the present work, we used the linear nearest neighbours search algorithm with the Euclidean distance as distance function. Leave-one-out cross-validation was used to select the best $\mathrm{k}$ value, with an upper limit of 35 nearest neighbours. The predictions from nearest neighbours were weighted according to the inverse distance.

In the best-case selection fusion scheme, we relied on the root mean square error (RMSE) of each phone prediction algorithm over the development data as the criterion for the selection of the best model for each case (Chen et al., 1998; Goubanova and King, 2008; Yamagishi et al., 2008). Specifically, the duration model prediction with the lowest RMSE for the cluster (vowels/consonants, phonetic category and individual phones) of each instance was selected.

\subsubsection{Databases and feature set}

In the evaluation experiments two databases were used: the American-English speech database CSTR US KED TIMIT (CSTR, 2001) and the Modern Greek speech prosodic database, WCL-1 (Zervas et al., 2008). KED TIMIT consists of 453 phonetically balanced sentences (3400 words approximately) uttered by a Native American male speaker. The WCL-1 prosodic database consists of 5500 words distributed in 500 paragraphs, uttered by a female professional radio actress. 
In this work, a number of features, which have been reported successful in the literature (Crystal and House, 1988; Campbell, 1992; Klatt, 1987; Goubanova and King, 2008; Riley, 1992; van Santen, 1994), are considered for the task of phone duration modelling. From each utterance we computed 33 features, and for some of them we also used their temporal neighbours, defined on the level of the respective feature, i.e. phone-level, syllable-level, word-level. The overall size of the feature vector, which was used for the individual phone duration models, including the aforementioned features and their temporal context information as reported above (one or two previous and next instances on the level of the respective feature, phonelevel, syllable-level, word-level) is 93 . These features were described in detail above in Section 4.1.

In all experiments an experimental protocol based on 10-fold cross-validation was followed. Specifically, in each fold the training data were split in two portions, the training dataset and the development dataset. The former, amounting to approximately $60 \%$ of the full dataset, was used for the training of the individual phone duration predictors, and the latter, amounting to approximately $30 \%$ of the full dataset, for the training of the fusion algorithm. Furthermore, the test dataset, amounting to approximately $10 \%$ of the full dataset, was used for evaluating the performance of the eight individual duration prediction algorithms, as well as the performance of the fusion scheme.

\subsubsection{Performance evaluation metrics}

The experimental results were evaluated using the two most commonly used figures of merit, namely the mean absolute error (MAE) and the root mean squared error (RMSE), between the predicted duration and the actual (reference) duration of each phone (Chen et al., 1998; Goubanova and King, 2008; van Santen, 1992; Yamagishi et al., 2008). These figures of merit were described in detail earlier in Section 4.1.2.3. 


\subsubsection{Experimental results}

In the following, the evaluation results for the twelve fusion algorithms outlined in Section 4.2, is reported. In Table 4.4, the results obtained in the evaluation of the average linear combination and the best-case selection techniques for different cases: overall performance, vowel/consonant categories, phonetic categories and individual phones are presented. The results for the best individual phone duration prediction model (SMOreg) are duplicated from Table 4.1 for the purpose of direct comparison. The average and the best-case selection techniques are considered as intuitive reference points against which the performance of the other ten fusion algorithms, evaluated here, is compared. As can be seen in Table 4.4 (a), for KED TIMIT, and in Table 4.4 (b), for the WCL-1 database, in the cases of vowel/consonant categories and phonetic categories the best-case selection algorithm did not offer advantage over the best individual model, SMOreg, since the SMOreg outperforms the other individual predictors in all categories (Table 4.2). As discussed above the only exception is Affricates on KED TIMIT, where the additive regression with REPTrees (Add. Regr. REPTrees) algorithm is the best, but is not sufficient for significant advantage of the fusion scheme.

Concerning the clustering according to individual phones, the best-case selection method slightly outperformed the best individual duration model on both databases. In detail, the best-case selection outperformed the SMOreg model by approximately $0.2 \%$ and $0.9 \%$ in terms of MAE and RMSE on the KED TIMIT database, and by approximately $0.6 \%$ and $0.5 \%$ on the WCL- 1 database. As can be seen in Table 4.3, this is owed to the fact that, for both databases, in approximately $35-40 \%$ of the phones the best performing algorithm is not the SMOreg. Consequently, the best-case selection fusion scheme outperforms the best individual phone duration prediction model (SMOreg). 
Table 4.4. Mean Absolute Error (MAE), standard deviation of absolute error (STD of AE) and Root Mean Square Error (RMSE) in milliseconds, for the fusion scheme, implemented with the average linear combination and the best-case selection fusion algorithms on: (a) the KED TIMIT database, and (b) the WCL-1 database.

(a) results on the KED TIMIT database

\begin{tabular}{lccc}
\hline Fusion algorithms on the KED TIMIT database & $\boldsymbol{M A E}(\boldsymbol{m s})$ & $\boldsymbol{S T D}$ of $\boldsymbol{A E}(\boldsymbol{m s})$ & $\boldsymbol{R M S E}(\boldsymbol{m s})$ \\
\hline Overall (average linear combination) & 15.32 & 14.01 & 20.76 \\
Vowel/consonant (best-case selection) & 14.95 & 14.11 & 20.56 \\
Phonetic category (best-case selection) & 14.94 & 14.11 & 20.54 \\
Phone (best-case selection) & 14.92 & 13.87 & 20.37 \\
\hline No fusion - best individual model, SMOreg & 14.95 & 14.11 & 20.56 \\
\hline
\end{tabular}

(b) results on the WCL-1 database

\begin{tabular}{lccc}
\hline Fusion algorithms on the WCL-1 database & MAE (ms) & STD of $\boldsymbol{A E}(\boldsymbol{m s})$ & $\boldsymbol{R} M \boldsymbol{H} \boldsymbol{E}(\boldsymbol{m s})$ \\
\hline Overall (average linear combination) & 16.91 & 18.72 & 25.29 \\
Vowel/consonant (best-case selection) & 16.78 & 18.81 & 25.21 \\
Phonetic category (best-case selection) & 16.78 & 18.81 & 25.21 \\
Phone (best-case selection) & 16.68 & 18.65 & 25.08 \\
\hline No fusion - best individual model, SMOreg & 16.78 & 18.81 & 25.21 \\
\hline
\end{tabular}

In Table 4.5, the results for the remaining ten fusion algorithms (refer to Section 4.2): the Linear Regression, the $m 5 p$ model tree, the $m 5 p R$ regression tree, the additive regression algorithms based on $\mathrm{m} 5 \mathrm{pR}$ and REPTrees (Add. Regr. $m 5 p R$ and Add. Regr. REPTrees), the bagging algorithms based on $\mathrm{m} 5 \mathrm{pR}$ and REPTree (Bagging m5pR and Bagging REPTrees), the instance based learning (IBK), the support vector regression (SVR) which implements the sequential minimal optimization (SMO) algorithm (SMOreg), and the radial basis function neural network $(R B F N N)$ are presented. 
Table 4.5. Mean Absolute Error (MAE), standard deviation of absolute error (STD of AE) and Root Mean Square Error (RMSE) in milliseconds, for the various fusion techniques on: (a) the KED TIMIT database, and (b) the WCL-1 database.

(a) results on the KED TIMIT database

\begin{tabular}{lccc}
\hline KED TIMIT database & MAE $(\boldsymbol{m s})$ & STD of $\boldsymbol{A E}(\boldsymbol{m s})$ & $\boldsymbol{R} \boldsymbol{M S E}(\mathbf{m s})$ \\
\hline SMOreg & $\mathbf{1 4 . 6 6}$ & $\mathbf{1 3 . 8 2}$ & $\mathbf{2 0 . 1 4}$ \\
IBK & 15.19 & 14.69 & 21.02 \\
Linear Regression & 15.49 & 14.45 & 21.18 \\
RBFNN & 15.53 & 14.49 & 21.24 \\
m5p & 15.56 & 14.60 & 21.34 \\
Add. Regr. m5pR & 15.72 & 14.94 & 21.69 \\
Add. Regr. REPTrees & 15.79 & 14.94 & 21.74 \\
Bagging m5pR & 15.81 & 15.09 & 21.86 \\
Bagging REPTrees & 15.88 & 15.15 & 21.95 \\
m5pR & 15.97 & 15.28 & 22.10 \\
\hline No fusion - best individual model, & 14.95 & 14.11 & 20.56 \\
SMOreg & & &
\end{tabular}

(b) results on the WCL-1 database

\begin{tabular}{lccc}
\hline WCL-1 database & MAE $(\mathbf{m s})$ & STD of $\boldsymbol{A E}(\mathbf{m s})$ & $\boldsymbol{R M S E}(\mathbf{m s})$ \\
\hline SMOreg & $\mathbf{1 6 . 3 5}$ & $\mathbf{1 8 . 5 9}$ & $\mathbf{2 4 . 7 6}$ \\
IBK & 16.98 & 18.85 & 25.47 \\
RBFNN & 17.34 & 19.51 & 26.10 \\
Add. Regr. m5pR & 17.69 & 19.84 & 26.58 \\
Bagging m5pR & 17.72 & 19.84 & 26.60 \\
m5p & 17.84 & 20.51 & 27.18 \\
m5pR & 17.91 & 20.00 & 26.85 \\
Bagging REPTrees & 17.99 & 20.45 & 27.23 \\
Add. Regr. REPTrees & 18.00 & 20.56 & 27.32 \\
Linear Regression & 18.32 & 20.19 & 27.26 \\
\hline No fusion - best individual model, & 16.78 & 18.81 & 25.21 \\
SMOreg & &
\end{tabular}

The best fusion result is shown in bold. For the reason of comparison, in Table 4.5 the results for the best individual phone duration model, SMOreg are duplicated. As can be seen from the results on the KED TIMIT (Table 4.5 (a)) and the WCL-1 (Table 4.5 (b)) databases, the SMOreg fusion model outperformed all the other fusion models that were evaluated here. It is also noteworthy to mention that only the SMOreg fusion model outperformed the best individual duration prediction model. For more convenience for the reader, the results for the SMOreg fusion model and the SMOreg individual model are presented in Fig. 4.3. Specifically, the SMOreg fusion model outperformed the individual SMOreg predictor by approximately $1.9 \%$ and $2.0 \%$ in terms of MAE and RMSE on KED TIMIT, and by approximately $2.6 \%$ and $1.8 \%$ on 
the WCL-1 database, respectively. Furthermore, it should be pointed out that the SMOreg fusion model apart from reducing the overall error also reduced the outliers. Specifically, in comparison to the best individual predictor, i.e. the SMOreg model, the SMOreg fusion model reduced the standard deviation of the absolute error (STD of AE), by approximately $2.1 \%$ on KED TIMIT and by approximately $1.2 \%$ on the WCL-1 database, respectively.

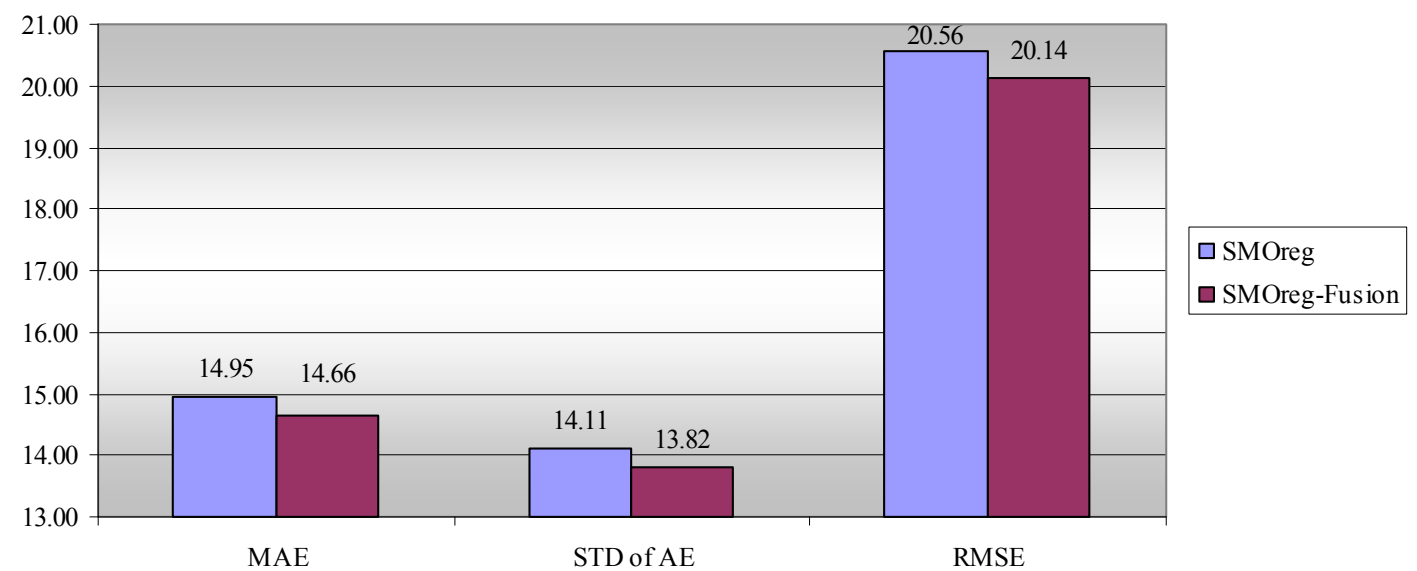

(a) results on the KED TIMIT database

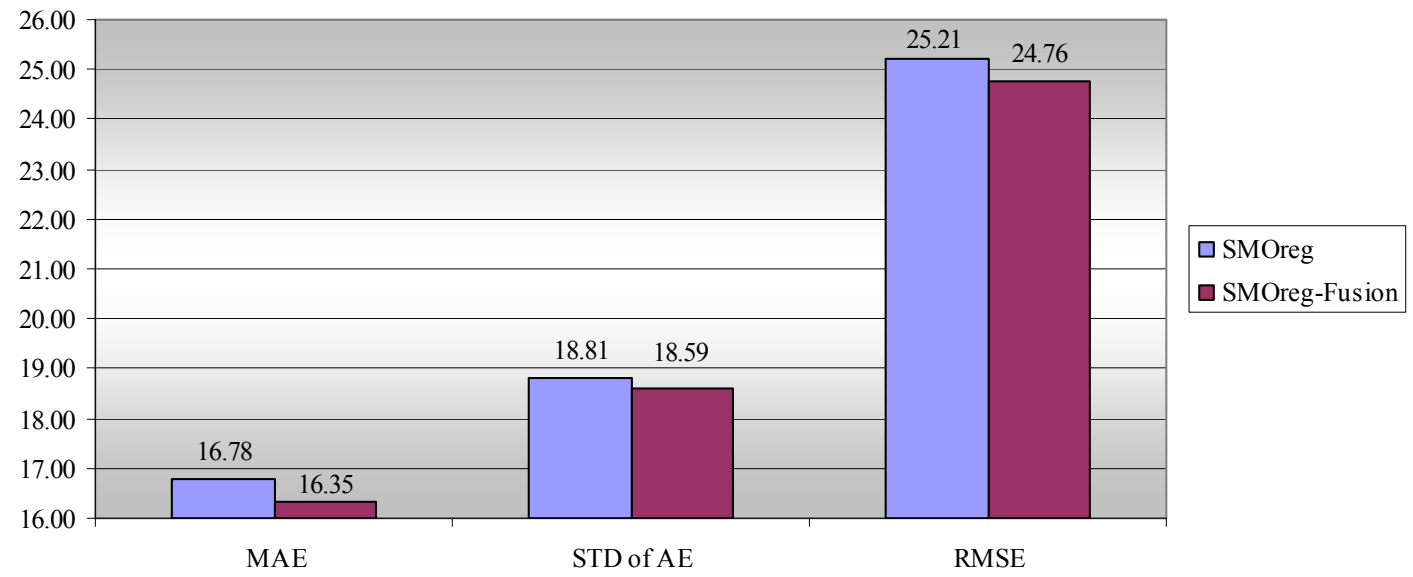

(b) results on the WCL-1 database

Fig. 4.3. Mean Absolute Error (MAE), standard deviation of absolute error (STD of AE) and Root Mean Square Error (RMSE) in milliseconds, for the individual SMOreg, and the fusion SMOreg-Fusion models on: (a) the KED TIMIT database, and (b) the WCL-1 database.

Finally, in order to investigate the statistical significance of the difference between the results for the best individual phone duration model (SMOreg) and the results for 
the best fusion scheme (SMOreg fusion algorithm) the Wilcoxon test (Wilcoxon, 1945) was carried out. The Wilcoxon test showed that on both databases, the difference between the results for the best individual model and these for the fusion scheme is statistically significant. Specifically, for a significance level of 0.05 the Wilcoxon test estimated a p-value equal to $5.77 \mathrm{e}^{-09}$ and $3.5 \mathrm{e}^{-11}$ on KED TIMIT and WCL-1 databases, respectively. Consequently, the fusion scheme contributes to the improvement of the accuracy of phone duration prediction, in comparison to best predictor among all evaluated individual phone duration prediction models.

\subsubsection{Conclusions}

Here, the accuracy of the proposed fusion scheme was studied. This scheme combines predictions from multiple individual phone duration models, operating on a common input, taking advantage of the observation that different prediction algorithms perform better in different situations. The experimental validation demonstrated that the fusion scheme improves the accuracy of phone duration prediction. The SVM-based fusion algorithm was found to outperform all other fusion techniques. Specifically, the fusion scheme based on the SVM regression (SMOreg) algorithm outperformed the best individual predictor (SVM regression - SMOreg) by approximately $1.9 \%$ and $2.0 \%$ in terms of relative reduction of the mean absolute error and root mean square error respectively, on the KED TIMIT database, and by $2.6 \%$ and $1.8 \%$ on the WCL-1 database, respectively.

\subsection{Two-stage prosody modelling with feature construction and feature vector extension}

In this section, the third proposed scheme is presented and evaluated: a two-stage phone duration modelling scheme, which is applicable to improved prosody modelling in speech synthesis systems. This scheme builds on a number of independent phone duration models implemented here as feature constructors (FCs) employed in the first stage, and a phone duration model (PDM), which operates on an 
extended feature vector in the second stage. The feature vector, which acts as input to the first stage, consists of numerical and non-numerical linguistic features extracted from text. The extended feature vector is obtained by appending the phone duration predictions estimated by the FCs to the initial feature vector. Experiments on the American-English KED TIMIT and on the Modern Greek WCL-1 databases validated the advantage of the proposed two-stage scheme improving prediction accuracy over the best individual predictor, and to the fusion scheme which just fuses the first-stage outputs, described previously in Section 4.2. Specifically, when compared to the best individual predictor, a relative reduction in the mean absolute error and the root mean square error of $3.9 \%$ and $3.9 \%$ on KED TIMIT, and of $4.8 \%$ and $4.6 \%$ on WCL-1 database, respectively, is observed. The improved accuracy of phone duration modelling contributes to a better control of the prosody, and thus quality of synthetic speech.

\subsubsection{Introduction}

The present work was inspired by studies on processing, combining or transforming an initial feature set to a new set of features (Breiman et al., 1984; Matheus and Rendell, 1989; Pagallo and Haussler, 1990; Watanabe and Rendell, 1991; Flach and Lavrac, 2000; De Jong et al., 1992; Heath et al., 1993; Quinlan, 1993; Ragavan et al., 1993; Wenk and Michalski, 1994; Hu and Kibler, 1996; Zheng, 1996). This procedure, referred to as feature construction, can lead either to the reduction of the complexity of the feature space (Piramuthu and Sikora, 2009) or to the enrichment of the initial feature space with additional features (Koller and Sahami, 1996; Dash and Liu, 1997). Feature construction uses one or more operators (Boolean expressions, logical rules, etc.) to combine two or more features of the initial feature set creating a new feature, or to transform features of the initial feature set, creating new ones. 


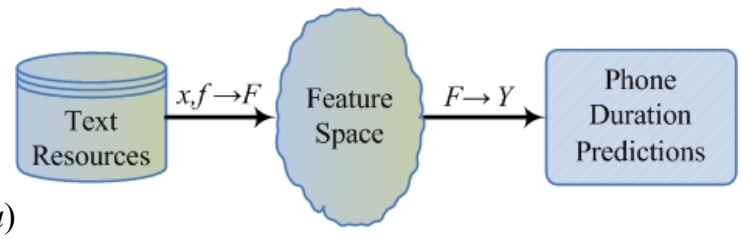

(a)

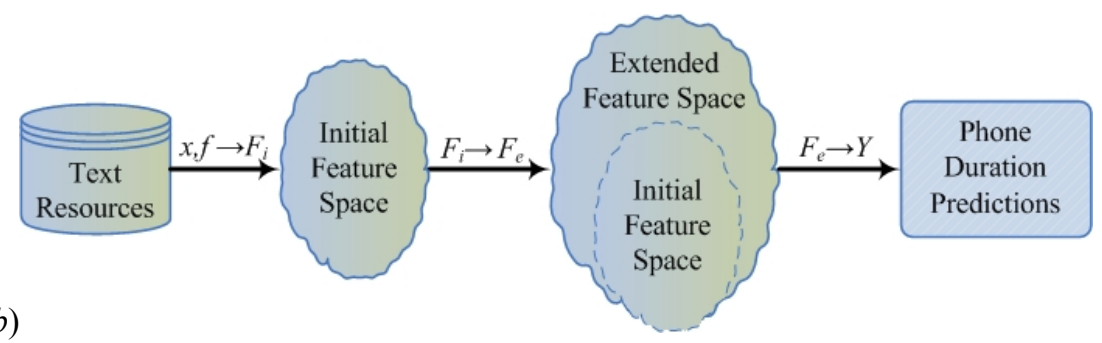

Fig. 4.4. Phone duration prediction: (a) the classical approach, (b) two-stage approach involving intermediate feature vector extension step.

In this work, a phone duration modelling approach, which incorporates a feature vector extension (FVE) stage, implemented through a feature construction process, illustrated in Fig. 4.4 (b), is proposed. In contrast to the classical approach (Fig. 4.4 (a)), which directly uses feature vectors extracted from data to predict the phones' durations, the proposed two-stage approach incorporating a FVE stage, attempts to enrich the initial feature space with newly constructed features, and consequently predict the phones' durations. This scheme builds on a number of phone duration models operating here as feature constructors (FCs) employed in the first stage, and a phone duration model (PDM) in the second stage, which operates on the extended feature vector. The FCs operate on a common input, the initial feature vector, constituted of linguistic features extracted only from text. The extended feature vector is obtained by appending the phone duration predictions estimated by the independent FCs to the initial feature vector. The newly constructed features capture the dependency between the initial features and the actual phones' durations in a manner that depends on the modelling technique and the training strategy employed in each of the FCs. In order for the FVE process to be beneficial in terms of overall phone duration prediction accuracy, it is essential that the FCs are based on various machine learning techniques. These techniques must rely on different assumptions and mapping functions of the feature space to the target space. In this study the phone duration models presented and evaluated in Section 4.1 are implemented here as FCs. 
This proposed scheme, the two-stage phone duration modelling scheme with the FVE stage, has not been studied previously on the task of prosody modelling.

\subsubsection{Two-stage scheme with feature construction and feature vector extension}

The two-stage phone duration modelling scheme is based on the use of multiple independent FCs in the first stage and a single PDM in the second stage, which operates on an extended feature vector. This two-stage scheme is based on the following logic of operation:

(i) the independent FCs show different performance on the phone duration modelling task, i.e. they err in a different manner. In order for this statement to be valid, we specify two criteria for selecting the PDMs that will serve as FCs: (a) the candidate models have to demonstrate state-of-the-art performance and/or to have advantage for specific category of units, and (b) the input feature vector, the machine learning technique or the training strategy have to be different from the other models that are already selected for the first stage.

(ii) the proposed two-stage scheme is expected to offer advantageous phone duration prediction accuracy, when compared to the best individual model, due to the benefits of fusion. These benefits are based on the known advantage that the fusion of the scores of multiple predictors, offers (Wolpert, 1992; Hashem and Schmeiser, 1995; Kittler et al., 1998; Kuncheva, 2002; Lazaridis et al., 2010; Mporas et al., 2010).

(iii) the proposed two-stage scheme with FVE would improve the phone duration prediction accuracy when compared to a baseline two-stage scheme which just fuses the outputs of the first stage predictors, i.e. without implementing FVE, described in Section 4.2. This advantage should come from extending the initial feature vector with the newly constructed features. These features convey information about the mapping of the initial feature space to the target "phone duration" space, since they offer independent projections between these two spaces. These projections, although might be considered as noisy, error-prone, imperfect, and correlated with the initial feature vector, are expected to facilitate the PDM in the second stage. This is due to their 
different perspective and to the independent mapping between the input and output, which assists in correcting anomalies.

In Fig. 4.5, the block diagram of the proposed two-stage phone duration modelling scheme, is presented. Following the criteria formulated in (i), the availability of numerous independent PDMs operating as FCs, is assumed. As mentioned previously, the FCs should be implemented with different machine learning techniques or trained with different strategies and could operate on different subsets of features, which represent the data in a complementary way or on a common input feature vector. As presented in the figure, in the following the case where all the FCs operate on a common input, is considered. This feature vector is composed of linguistic features extracted only from text since text is the input in TTS systems. In the following subsections the proposed scheme is described in detail.

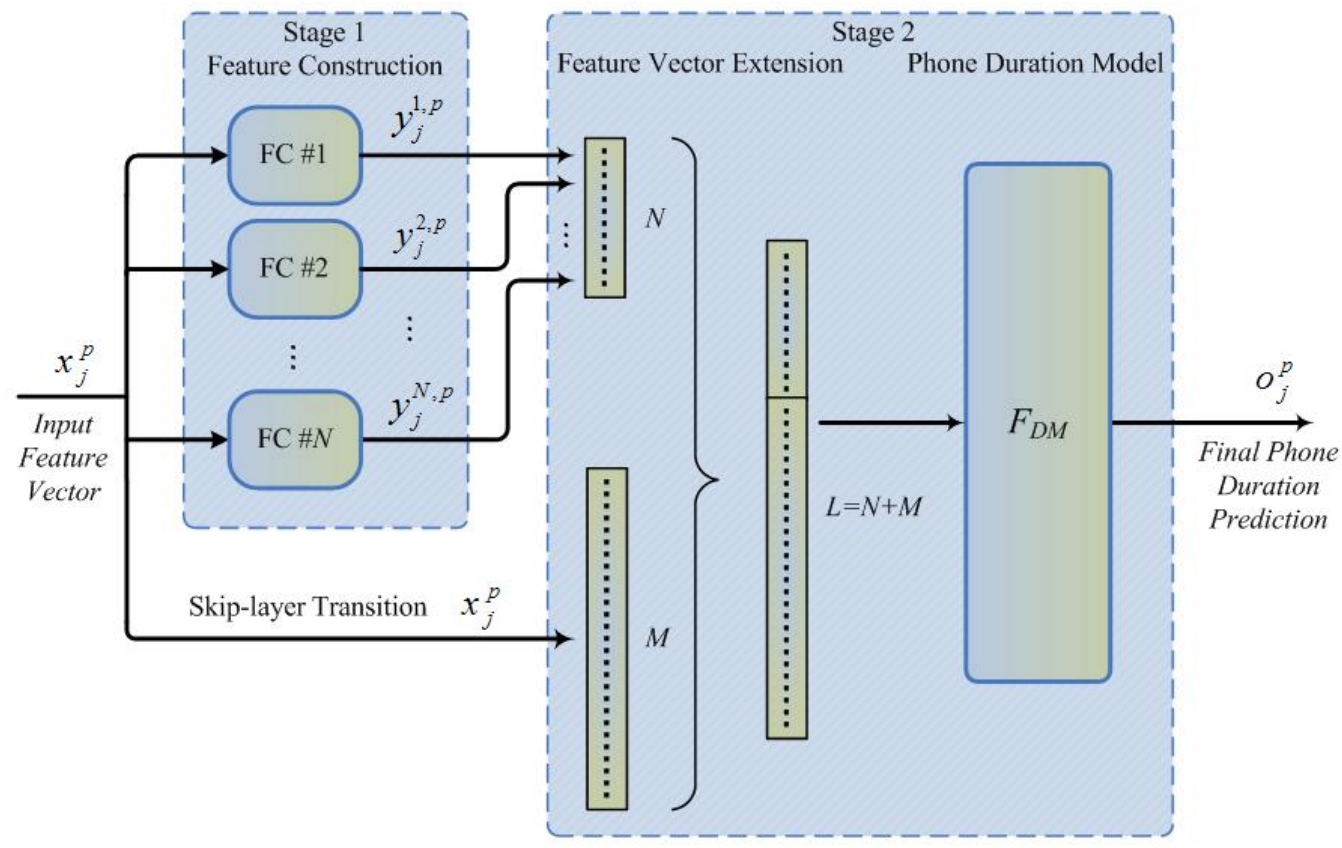

Fig. 4.5. Block diagram of the two-stage phone duration modelling scheme, which involves feature construction and feature vector extension.

\subsubsection{Feature constructors}

The training of the proposed two-stage phone duration modelling scheme depends on two non-overlapping datasets: the training and the development sets. Initially, the independent FCs are trained using the training dataset, and consequently the trained 
FCs are used to process the development dataset in order to produce new features. These newly constructed features, which are in fact phone duration predictions, are appended to the initial feature vector, which contains linguistic features of several speech representation levels, e.g. the phonetic, phonological and morphosyntactic. The composite feature vector obtained after this merging, referred to as extended feature vector, is employed for the training of the PDM in the second stage of the scheme.

The training of the FCs can be formalized as follows: Let us define a set of $N$ independent FCs, which operate on a common input. Furthermore, let us define a $M$ dimensional feature vector, $X_{j}^{p}$, which consists of numeric and non-numeric features, and which acts on the FCs input. Here, the subscript and superscript indexes of $X_{j}^{p}$ stand for the instance $j$ of training data for phone $p$. The training dataset, consisting of all feature vectors are used together to train each FC. The trained FCs are next used to process the data of the development dataset and the outcome of this processing is phone duration predictions, $y_{j}^{n, p}=B_{p}^{n}\left(X_{j}^{p}, b_{n}(p)\right)$, which serve as the newly constructed features. Here, each of the $N$ outputs, $y_{j}^{n, p}(n=1 \ldots N$, where $n$ is the index of each FC) is a real number corresponding to predicted phone duration value, while $B_{p}^{n}$ and $b_{n}(p)$ stand for the mapping function and the phone-dependent parameters of the $n t h \mathrm{FC}$, for phone $p$, respectively. The outputs of the FCs are concatenated to form the $N$-dimensional feature vector $Y_{j}^{p}=\left[y_{j}^{1, p}, \ldots, y_{j}^{n, p}, \ldots, y_{j}^{N, p}\right]$, which is next appended to the initial feature vector, $X_{j}^{p}$, to obtain the extended feature vector acting as input to the second stage.

\subsubsection{Phone duration model}

The PDM in the second stage is trained with the extended feature vectors obtained after processing the development dataset. In detail, the initial feature vector, $X_{j}^{p}$, and the outputs of the $N$ FCs, $Y_{j}^{p}$, are concatenated to form the extended feature vector $Z_{j}^{p}=\left[X_{j}^{p} \| Y_{j}^{p}\right]^{T}$ with dimensionality $L=M+N$. The extended feature vector $Z_{j}^{p}$ 
together with the ground truth labels from the database are used for training the PDM, denoted as $F_{D M}$. Once the $F_{D M}$ is trained, the two-stage phone duration modelling scheme is ready for operation.

During run-time operation of the two-stage scheme (cf. Fig. 4.5), the input data are processed as follows: An $M$-dimensional input feature vector $X_{j}^{p}$, for the $j t h$ instance, appears as input to all the FCs. The outputs of the FCs, i.e. the phone duration predictions, $y_{j}^{n, p}$, are the newly constructed features. They are appended to the initial feature vector $X_{j}^{p}$ used in the first stage, and the $L$-dimensional extended feature vector obtained to this end is fed as input to the PDM in the second stage. The output of the model $F_{D M}$ is the final phone duration prediction, $o_{j}^{p}=F_{D M}^{p}\left(Z_{j}^{p}, f_{n}(p)\right)$, where $F_{D M}^{p}$ is the mapping function for phone $p$, and $f_{n}(p)$ are the phone-dependent parameters of $F_{D M}$.

\subsubsection{Experimental setup}

In order to investigate the practical usefulness of the proposed two-stage scheme, a number of independent models, which were employed to operate as FCs in the first stage is trained and various implementations of the PDM in the second stage are investigated. The machine learning algorithms used in the implementation of the FCs and PDM are described in the following two subsections.

\subsubsection{Feature constructors}

Eight independent phone duration modelling methods, which are well known and have been used successfully over the years in different modelling tasks, to be used as FCs, are considered. These models are the ones used in the proposed fusion scheme as individual phone duration models (Section 4.2.3.1). In brief these are:

(i) the linear regression (LR) (Witten and Frank, 2005) using Akaike's Information Criterion (AIC) (Akaike, 1974) in backward stepwise selection 
(BSS) (Kohavi and John, 1997) procedure eliminating unnecessary variables of the training data,

(ii) the m5p model tree, using a linear regression function on each leaf, and the $\mathrm{m} 5 \mathrm{pR}$ regression tree, using a constant value on each leaf node instead (Quinlan, 1992; Wang and Witten, 1997).

(iii) two additive regression algorithms (Friedman, 2002) and two bagging algorithms (Breiman, 1996) were used, by using two different regression trees (m5pR and REPTrees) (Kaariainen and Malinen, 2004; Quinlan, 1992; Wang and Witten, 1997) as base classifiers in each case.

(iv)Finally, the support vector regression (SVR) model (Platt, 1999), which employs the sequential minimal optimization (SMO) algorithm for training a support vector classifier (Smola and Scholkopf, 1998), was used. In our experiments the RBF kernel was used as mapping function.

The motivation to select these machine learning algorithms was based on previous research (Yamagishi et al., 2008; Iwahashi and Sagisaka, 2000; Takeda et al., 1989; Riley, 1992; Lee and Oh, 1999b), where these algorithms were reported successful on phone duration modelling. Once these eight models are built with different machine learning techniques and have different training strategy, they well conform to the criteria formulated in Section 4.3.2.

\subsubsection{Phone duration model in the second stage}

In order to select the most advantageous algorithm for the PDM in the second stage, we experimented with ten different machine learning algorithms. These include the eight algorithms outlined in the previous section, along with (i) the radial basis function neural network (RBFNN) with Gaussian kernel (Park and Sandberg, 1993), and (ii) the instance-based algorithm (IBK) (Aha and Kibler, 1991) which is a knearest neighbours' classifier. These models are the ones reported in Section 4.2.3.2, used as fusion models in the fusion scheme. 


\subsubsection{Databases and feature set}

The evaluation for the phone duration modelling task was carried out on two databases: the American English speech database, CSTR US KED TIMIT (CSTR, 2001), and the Modern Greek speech prosodic database, WCL-1 (Zervas et al., 2008). KED TIMIT consists of 453 phonetically balanced sentences $(3400$ words approximately) uttered by a Native American male speaker. The WCL-1 prosodic database consists of 5500 words distributed in 500 paragraphs, uttered by a female professional radio actress (see Section 4.1.2.2). In all experiments with both databases, the manually labelled phones' durations were used as the ground truth reference durations.

From each utterance of the database, for each instance of the utterance which corresponds to a phone, 33 features were computed. The temporal neighbours of some of these features, defined on the level of the respective feature, i.e. phone-level, syllable-level and word-level, were also used. The overall size of the initial feature vector is 93, including the aforementioned features and their temporal context information as reported above (one or two previous and next instances on the level of the respective feature, phone-level, syllable-level and word-level). These features are reported in detain in Section 4.1.2.2.

For the purpose of comparison, the phone duration prediction accuracy of the proposed two-stage scheme with FVE is evaluated in contrast to (i) the accuracy of the best independent FC method, and (ii) the proposed fusion scheme (Section 4.2), which in this work is referred to as fusion of the constructed features (FCF). The FCF scheme is equivalent to a direct fusion of the predictions of the FCs, since only the outputs of the FCs are used as feature vector for the PDM in the second stage. This scheme will be considered as the baseline to which the performance of the proposed FVE scheme is compared to.

In all experiments an experimental protocol based on 10-fold cross-validation was followed. Specifically, in each fold the training data were split in two non-overlapping portions: the training dataset and the development dataset. The training dataset, amounting to approximately $60 \%$ of the full dataset, was utilized for the training of the first-stage FCs and the development dataset, amounting to approximately $30 \%$ of the full dataset, was used for the training of the PDM in the second stage. Furthermore, the test dataset, amounting to approximately $10 \%$ of the full dataset, was 
used for evaluating the performance of the eight individual FCs, as well as the overall performance of the two-stage scheme.

\subsubsection{Performance evaluation metrics}

The experimental results are reported in terms of the two most commonly used figures of merit, namely the mean absolute error (MAE) and the root mean squared error (RMSE), between the predicted duration and the actual (reference) duration of each phone (Yamagishi et al., 2008; Chen et al., 2003; Chen et al., 1998; Goubanova and King, 2008; Krishna and Murthy, 2004; Lee and Oh, 1999a; Riley, 1992). Due to the squaring of values in the RMSE, the large errors (outliers) are weighted heavily, which makes this figure of metric more sensitive to outliers than the MAE (Witten and Frank, 2005). This sensitivity of the RMSE makes it a more illustrative measurement concerning the gross errors, when compared to the MAE. These figures of merit were described in detail earlier in Section 4.1.2.3.

\subsubsection{Experimental results}

All the FC models, which are the individual phone duration models, were reported evaluated and discussed in Section 4.1. In this section, we investigate the accuracy of the proposed two-stage phone duration modelling scheme with FVE. This scheme is compared to the baseline fusion scheme (Section 4.2), i.e. FCF, where the initial feature vector is not propagated to the second stage and only the outputs of the FCs are used as input to the PDM in the second stage. In the following ten different implementations of the PDM in the second stage are considered, and their performance both with and without FVE is evaluated.

In Table 4.6, the results for the ten algorithms outlined in Section 4.3.3.2, which are the $L R$, the $m 5 p$ model tree, the $m 5 p R$ regression tree, the two additive regression algorithms based on $\mathrm{m} 5 \mathrm{pR}$ and REPTrees (Add. Regr. m5pR and Add. Regr. REPTrees), the two bagging algorithms based on m5pR and REPTrees (Bagg. m5pR and Bagg. REPTrees), the instance-based learning (IBK), the support vector regression (SVR) with sequential minimal optimization (SMO) training referred here 
as SMOreg, and the radial basis function neural network (RBFNN) are presented. In all cases the best results are shown in bold. Again for the reason of comparison, in Table 4.6 the experimental results for the best individual FC (SMOreg) are also presented.

Table 4.6. Mean Absolute Error (MAE), standard deviation of absolute error (STD of AE) and Root Mean Square Error (RMSE) in milliseconds, for the baseline FCF scheme and for the proposed FVE scheme on: (a) the KED TIMIT database, and (b) the WCL-1 database.

(a) results on the KED TIMIT database

\begin{tabular}{lcccccc}
\hline \multirow{2}{*}{ PDM algorithms } & \multicolumn{3}{c}{ baseline, FCF } & \multicolumn{4}{c}{ proposed scheme, FVE } \\
\cline { 2 - 7 } & $\boldsymbol{M A E} \boldsymbol{S T D}$ of $\boldsymbol{A E}$ & $\boldsymbol{R M S E}$ & $\boldsymbol{M A E}$ & $\boldsymbol{S T D}$ of $\boldsymbol{A E} \boldsymbol{R M S E}$ \\
\hline Add. Regr. m5pR & 15.72 & 14.94 & 21.69 & 15.74 & 14.92 & 21.69 \\
Add. Regr. REPTrees & 15.79 & 14.94 & 21.74 & 15.60 & 14.76 & 21.47 \\
Bagg. m5pR & 15.81 & 15.09 & 21.86 & 15.83 & 15.08 & 21.86 \\
Bagg. REPTrees & 15.88 & 15.15 & 21.95 & 16.26 & 15.52 & 22.48 \\
IBK & 15.19 & 14.69 & 21.02 & 17.41 & 15.88 & 23.57 \\
LR & 15.49 & 14.45 & 21.18 & 15.40 & 14.40 & 21.08 \\
m5p & 15.56 & 14.60 & 21.34 & 15.45 & 14.48 & 21.17 \\
m5pR & 15.97 & 15.28 & 22.10 & 15.95 & 15.25 & 22.06 \\
RBFNN & 15.53 & 14.49 & 21.24 & 21.28 & 18.37 & 28.11 \\
SMOreg & 14.66 & 13.82 & 20.14 & $\mathbf{1 4 . 3 6}$ & $\mathbf{1 3 . 5 6}$ & $\mathbf{1 9 . 7 5}$ \\
\hline best FC (SMOreg) & - & - & - & 14.95 & 14.11 & 20.56 \\
\hline
\end{tabular}

(b) results on the WCL-1 database

\begin{tabular}{lcccccc}
\hline \multirow{2}{*}{ PDM algorithms } & \multicolumn{3}{c}{ baseline, FCF } & \multicolumn{4}{c}{ proposed scheme, FVE } \\
\cline { 2 - 7 } & MAE & STD of AE & RMSE & MAE & STD of AE RMSE \\
\hline Add. Regr. m5pR & 17.69 & 19.84 & 26.58 & 17.75 & 19.93 & 26.69 \\
Add. Regr. REPTrees & 18.00 & 20.56 & 27.32 & 17.89 & 20.22 & 26.99 \\
Bagg. m5pR & 17.72 & 19.84 & 26.60 & 17.75 & 19.82 & 26.60 \\
Bagg. REPTrees & 17.99 & 20.45 & 27.23 & 18.15 & 20.51 & 27.39 \\
IBK & 16.98 & 18.85 & 25.47 & 18.53 & 20.29 & 27.48 \\
LR & 18.32 & 20.19 & 27.26 & 18.22 & 20.11 & 27.14 \\
m5p & 17.84 & 20.51 & 27.18 & 17.81 & 20.46 & 27.12 \\
m5pR & 17.91 & 20.00 & 26.85 & 17.98 & 20.02 & 26.91 \\
RBFNN & 17.34 & 19.51 & 26.10 & 21.29 & 21.43 & 30.21 \\
SMOreg & 16.35 & 18.59 & 24.76 & $\mathbf{1 5 . 9 7}$ & $\mathbf{1 8 . 3 4}$ & $\mathbf{2 4 . 0 4}$ \\
\hline best FC (SMOreg) & - & - & - & 16.78 & 18.81 & 25.21 \\
\hline
\end{tabular}

For more convenience for the reader, the results for the best individual FC (SMOreg) model, the baseline FCF SMOreg scheme and the FVE SMOreg scheme are presented in Fig. 4.6. Specifically, the baseline FCF scheme, implemented with the SMOreg model, outperformed the best individual FC, the SMOreg, by $1.9 \%$ and $2 \%$ in terms of MAE and RMSE on the KED TIMIT database (Fig. 4.6 (a)), and respectively by $2.6 \%$ and $1.8 \%$ on the WCL-1 database (Fig. 4.6 (b)). The proposed 
FVE scheme outperformed the best FC (SMOreg) on both KED TIMIT and WCL-1 databases only when the PDM in the second-stage is implemented with SMOreg. In terms of MAE and RMSE, the benefit of the FVE is shown by the accuracy improvement of $3.9 \%$ and $3.9 \%$ on the KED TIMIT database and of $4.8 \%$ and $4.6 \%$ on the WCL-1 database, respectively.

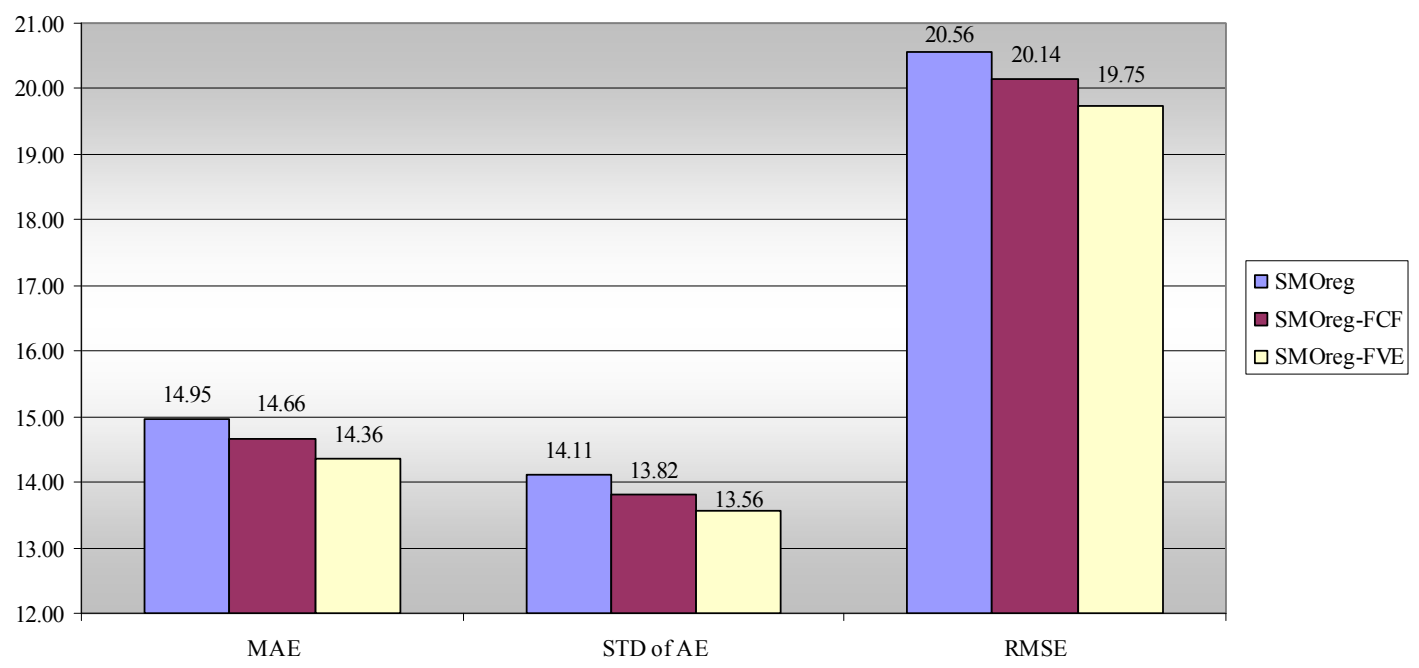

(a) results on the KED TIMIT database

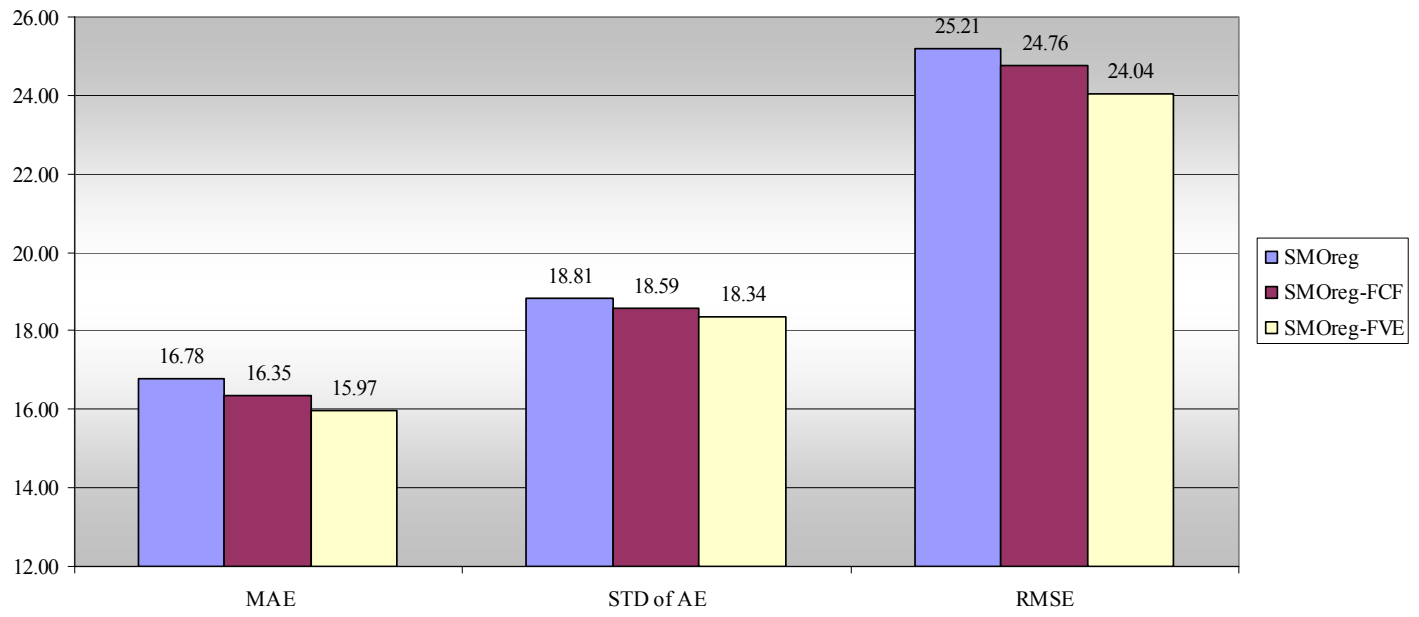

(b) results on the WCL-1 database

Fig. 4.6. Mean Absolute Error (MAE), standard deviation of absolute error (STD of AE) and Root Mean Square Error (RMSE) in milliseconds, for individual SMOreg-FC, the SMOreg-FCF and the SMOreg-FVE models on: (a) the KED TIMIT database, and (b) the WCL-1 database. 
Moreover, it should be mentioned that the proposed FVE scheme, apart from reducing the overall error also reduces the deviation of the outliers. In the case when the error distribution is Gaussian, the reduction in the standard deviation of the absolute error is correlated to the reduction of the outliers with respect to the model. As the results in Fig. 4.6 show, the proposed FVE scheme reduced the STD of AE in comparison to the best FC, the SMOreg, by approximately $3.9 \%$ on the KED TIMIT database and by approximately $2.5 \%$ on the WCL-1 database, respectively.

As already said, the FVE scheme outperforms the best individual FC, SMOreg, only when the PDM in the second-stage is implemented with SMOreg. This observation can be reasoned with the high dimensionality of the input feature vector (93 initial features +8 newly constructed $=103$ dimensions). This is because the other machine learning techniques, when employed as PDM in the second stage, do not build robust models from the available training data due to the curse of dimensionality. Since the support vector machines do not suffer from this problem they performed better than any of the other techniques evaluated here.

Finally, in order to investigate whether the differences in the accuracies between the best individual FC and the proposed FVE scheme, and between the baseline (FCF) and the proposed FVE scheme, are statistically significant, the Wilcoxon tests (Wilcoxon, 1945) were performed. On both databases, the Wilcoxon test showed that these differences are statistically significant, with the significance level of p-value $<$ 0.05. Thus, the proposed FVE scheme can be regarded as advantageous when compared both to the best individual FC and to the baseline FCF scheme.

\subsubsection{Additional experiments with feature ranking}

For further investigation of the effectiveness of the proposed FVE scheme, a subset selection on the extended feature vector was performed. For that purpose, firstly feature ranking with the Recursive Elimination of Features (RELIEF) algorithm (Kira and Rendell, 1992) was performed, and subsequently the top-20, top-50, top-80 ranked features were selected. This resulted in three subsets of the extended feature vector, which in the following we refer to as the Sets 20,50 and 80 , respectively. The entire extended feature vector is referred to as the full feature set. 
The feature selection was performed on a randomly selected subset, corresponding to $40 \%$ of the development dataset. The eight constructed features obtained from the FCs (evaluated in Section 4.1), were ranked in the top-13 features on KED TIMIT, and in the top-18 on the WCL-1 database. Thus, the feature ranking results confirm the importance of the newly constructed features and give explanation for their contribution to the reduction of the error rates, when they are used as extension of the initial feature vector.

Table 4.7. Mean Absolute Error (MAE), standard deviation of absolute error (STD of AE) and Root Mean Square Error (RMSE) in milliseconds, for the various feature subsets on the PDMs on: (a) the KED TIMIT database, and (b) the WCL-1 database.

(a) results on the KED TIMIT database

\begin{tabular}{|c|c|c|c|c|c|c|c|c|}
\hline \multirow[b]{2}{*}{ PDM algorithms } & \multicolumn{2}{|c|}{ PDM-Set 20} & \multicolumn{2}{|c|}{ PDM-Set 50} & \multicolumn{2}{|c|}{ PDM-Set 80} & \multicolumn{2}{|c|}{ PDM-Full set } \\
\hline & $M A E \begin{array}{c}S T D \\
\text { of } A E\end{array}$ & & $A E \begin{array}{c}S T D \\
\text { of } A E\end{array}$ & & $\begin{array}{l}S T D \\
\text { of } A E\end{array}$ & & $\begin{array}{c}S T D \\
\text { of } A E\end{array}$ & \\
\hline$p R$ & 15.7314 .93 & 9 & 15.714 .90 & 2 & 7114.90 & & 414.92 & 21.69 \\
\hline Add. Regr. R & 314.80 & 21 & 15.6614 .79 & 21. & 6314.76 & 21.49 & 6014.76 & 21.47 \\
\hline Bagg. $m 5 p R$ & 8 & & 15.08 & 21. & 215.08 & & .08 & 21.86 \\
\hline Bagg. REPTr & 2615.52 & 22. & .2715 .51 & 22.48 & 16.2615 .50 & 22.46 & 16.2615 .52 & 22.48 \\
\hline$I B K$ & 814.71 & 21 & 15.85 & 23.10 & 1115.78 & 23.28 & 17.4115 .88 & 23.57 \\
\hline$L R$ & 4014.42 & 21. & .3914 .41 & 21.0 & 3914.40 & 21.07 & 15.4014 .40 & 21.08 \\
\hline$m 5 p$ & 4814.47 & 21 & 5014.63 & 21.31 & .4614 .45 & 21.16 & 15.4514 .48 & 21.17 \\
\hline $25 p R$ & 9615.28 & 22.1 & .9615 .26 & 22.08 & .9515 .27 & 22.08 & 15.9515 .25 & 22.06 \\
\hline$R B F N N$ & 5114.81 & 22.18 & 18.8517 .01 & 25.39 & 20.3817 .83 & 27.08 & 21.2818 .37 & 28.11 \\
\hline SMOreg & 4.5413 .75 & 20.01 & 4.4413 .66 & 19.87 & 14.3713 .60 & 19.79 & 14.3613 .56 & 19.75 \\
\hline best FC (SMOreg) & - & - & - & - & - & - & 14.9514 .11 & 20.56 \\
\hline
\end{tabular}

(b) results on the WCL-1 database

\begin{tabular}{|c|c|c|c|c|c|c|c|c|}
\hline \multirow[b]{2}{*}{ PDM algorithms } & \multicolumn{2}{|c|}{ PDM-Set 20} & \multicolumn{2}{|c|}{ PDM-Set 50} & \multicolumn{2}{|c|}{ PDM-Set 80} & \multicolumn{2}{|c|}{ PDM-Full set } \\
\hline & $M A E \begin{array}{c}S T D \\
\text { of } A E\end{array}$ & & $\begin{array}{c}S T D \\
\text { of } A E\end{array}$ & & $A_{E} \begin{array}{c}S T D \\
\text { of } A E\end{array}$ & & $\begin{array}{c}S T D \\
\text { of } A E\end{array}$ & \\
\hline$\overline{A d d . R e g r . m 5 p R}$ & 17.7619 .92 & 26.69 & 17.7619 .98 & 26.74 & 17.7419 .89 & 26.65 & 17.7519 .93 & 26.69 \\
\hline Add. Regr. REPTrees & 17.9320 .57 & 27.28 & 17.9320 .41 & 27.17 & 17.9620 .57 & 27.31 & 17.8920 .22 & 26.99 \\
\hline Bagg. $m 5 p R$ & 17.7319 .83 & 26.60 & 17.7419 .84 & 26.62 & 17.7519 .83 & 26.61 & 17.7519 .82 & 26.60 \\
\hline Bagg. REPTrees & 18.1320 .53 & 27.39 & 18.1420 .54 & 27.40 & 18.1420 .54 & 27.41 & 18.1520 .51 & 27.39 \\
\hline$I B K$ & 17.2419 .32 & 25.90 & 17.9620 .05 & 26.92 & 18.1920 .20 & 27.18 & 18.5320 .29 & 27.48 \\
\hline$L R$ & 18.2020 .11 & 27.12 & 18.2120 .07 & 27.10 & 18.2320 .09 & 27.13 & 18.2220 .11 & 27.14 \\
\hline$m 5 p$ & 17.8920 .47 & 27.18 & 17.8320 .22 & 26.96 & 17.7820 .07 & 26.81 & 17.8120 .46 & 27.12 \\
\hline$m 5 p R$ & 17.9320 .01 & 26.87 & 17.9620 .01 & 26.89 & 17.9520 .02 & 26.89 & 17.9820 .02 & 26.91 \\
\hline$R B F N N$ & 17.9419 .77 & 26.70 & 19.8120 .76 & 28.69 & 20.6920 .99 & 29.47 & 21.2921 .43 & 30.21 \\
\hline SMOreg & 16.2818 .62 & 24.68 & 16.1718 .57 & 24.39 & 16.0518 .65 & 24.18 & 15.9718 .34 & 24.04 \\
\hline best FC (SMOreg) & - & - & - & - & - & - & 16.7818 .81 & 25.21 \\
\hline
\end{tabular}

In Table 4.7, the results for the proposed FVE scheme, when the second stage is fed with the feature subsets 20, 50 and 80, are presented. Here Table 4.7 (a) presents 
the results on the KED TIMIT database and Table 4.7 (b) on the WCL-1 database. For the reason of comparison, in the last three columns of Table 4.7, the experimental results for the full feature set are duplicated. As can be seen in the table, in the case of Set 20 , where only the top-20 features of the full feature set are fed to the second stage, the proposed FVE scheme with SMOreg in the second stage outperforms the best FC, the SMOreg model, by $2.7 \%$ and $2.7 \%$ in terms of MAE and RMSE, respectively, on the KED TIMIT database and by $3 \%$ and $2.1 \%$, respectively, on the WCL-1 database. However, the performance on Set 20 is worse than the one obtained on the full feature set. The same is valid for Set 50 and Set 80. In the case of Set 50, where the top-50 features are fed to the second stage, again only the proposed FVE scheme outperforms the best FC by $3.4 \%$ and $3.4 \%$ in terms of MAE and RMSE, respectively, on the KED TIMIT database, and by $3.6 \%$ and $3.3 \%$, respectively, on the WCL-1 database. Likewise, for the Set 80, the proposed FVE scheme outperforms the best FC by $3.9 \%$ and $3.7 \%$ in terms of MAE and RMSE, respectively, on KED TIMIT and by $4.4 \%$ and $4.1 \%$, respectively, on the WCL- 1 database.

Analyzing further the results shown in Table 4.7, it should be noted that the models based on the $I B K$ and the $R B F N N$ techniques showed a noticeable dropping in their accuracy as the number of the features in the feature vector was increasing (from Set 20 to the full feature set), increasing the MAE by $10.3 \%$ and $7.5 \%$ on the KED TIMIT and WCL-1 databases, respectively, for $I B K$ model, and by $28.9 \%$ and $18.7 \%$, respectively, for the RBFNN model. This decline in the phone duration prediction accuracy shows that the IBK (Hamraz and Feyzabadi, 2005) and RBFNN (Liu et al., 2007) models suffer from the curse of dimensionality (Hughes, 1968), and thus they do not handle well the increased dimensionality of the feature vector, given the predefined amount of the training data.

Furthermore, it is shown that for all the other machine learning techniques evaluated here, the increase in the feature vector dimensionality (from Set 20 to the full feature set) showed no significant improvement in the accuracy of the PDMs and in no case did any model, rather than the SMOreg, outperformed the best individual FC, SMOreg.

In conclusion, we can summarize that the experimental results confirmed the advantage of the proposed two-stage phone duration modelling scheme, which incorporates a number of FCs in the first stage and a SVM-based PDM in the second stage that operates on an extended feature vector. The proposed scheme contributes to 
a significant gain of accuracy, when compared (i) to the best individual FC, and (ii) to the case of simple fusion of the outputs of the FCs, i.e. without extension of the features. Finally, ranking the relevance of the individual features in the extended feature vector demonstrated the high relative importance of the newly constructed features, which well explains the observed accuracy gain.

\subsubsection{Conclusions}

The proposed two-stage phone duration modelling scheme, which relies on a number of independent feature constructors employed in the first stage and a phone duration model in the second stage, which operates on extended feature vector, was studied. This scheme takes advantage of the fact that different prediction algorithms operating on a common input can construct complementary features, which when appended to the initial feature vector contribute to the improvement of the overall phone duration prediction accuracy. The support vector regression was found to be the most appropriate implementation for the second-stage phone duration modelling in the proposed scheme. Specifically, the SMO regression model outperformed the best feature constructor by $3.9 \%$ and $3.9 \%$ in terms of mean absolute error and root mean square error, respectively, on the KED TIMIT database and by $4.8 \%$ and $4.6 \%$, respectively, on the WCL-1 database. The extended feature vector, consisting of the initial feature vector and the newly constructed features, was found advantageous over the three smaller subsets.

The improved accuracy of phone duration modelling obtained with the proposed two-stage scheme contributes to a better control of the prosody of synthetic speech and consequently to the improvement of the overall quality of synthetic speech. 


\section{Chapter 5}

\section{PROSODY MODELLING IN EMOTIONAL SPEECH}

Accurate modelling of prosody is prerequisite for the production of synthetic speech of high quality. Phone duration, as one of the key prosodic parameters, plays an important role for the generation of emotional synthetic speech with natural sounding. In this chapter, an overview of various phone duration modelling techniques is offered, and consequently ten models, based on decision trees, linear regression, lazy-learning algorithms and meta-learning algorithms, which over the past decades have been successfully used in various modelling tasks, are evaluated. Furthermore, the opportunity for performance optimization by applying two feature selection techniques, the RReliefF and the Correlation-based Feature Selection, on a large set of numerical and nominal linguistic features extracted from text, such as: phonetic, phonologic and morphosyntactic ones, which have been reported successful on the phone and syllable duration modelling task, is studied. The practical usefulness of these phone duration modelling techniques on a Modern Greek emotional speech database, which consists of five categories of emotional speech: anger, fear, joy, neutral, sadness is investigated. The experimental results demonstrated that feature selection significantly improves the accuracy of phone duration prediction regardless of the type of machine learning algorithm used for phone duration modelling. Specifically, in four out of the five categories of emotional speech, feature selection contributed to the improvement of the phone duration modelling, when compared to 
the case without feature selection. Finally, the first proposed model in this dissertation, support vector regression, is evaluated in the task of phone duration modelling in emotional speech, outperforming all the other models.

\subsection{Introduction to emotional speech synthesis}

Human-Computer interaction deals with the communication between human and machine, trying to imitate the processes that take place during the oral communication between two humans (Sears and Jacko, 2007). Speech synthesis, through text-tospeech (TTS) systems, plays a very important role in human-centred computing systems, being responsible for generating the system response through high-quality speech to the human-user.

Over the years, in the attempt to improve the quality (naturalness and intelligibility) of synthetic speech (Dutoit, 1997), various methods of speech synthesis, beginning from articulatory and formant synthesis and passing to diphone concatenative, have been introduced and developed (cf. (Taylor, 2009) and the references therein) in this area of HCI. With the collection and deployment of large speech databases, unit-selection corpus-based speech synthesis was introduced. Unitselection synthesis exploits many hours of speech containing hundreds or thousands of instances for each unit (e.g., phones or diphones) and produces the highest quality synthetic speech among all the aforementioned methods.

Today, the state-of-the-art speech synthesis systems produce synthetic speech of very high quality, making sometimes impossible for the listener to distinguish the synthetic speech from the real human speech. In addition, over the last 10 years, a large effort has been undertaken in order to produce even more natural and most importantly human-like synthetic speech reflecting different emotions. This attempt was focused on the development of emotional speech databases as well as the proper modelling of emotions through formant synthesis, diphone concatenative synthesis and unit-selection corpus-based approaches (cf. (Schröder, 2009) and the references therein).

Formant synthesis in based on the proper modelling and prediction directly of the acoustic data corresponding to speech through the implementation of a set of rules 
developed by experts. On one hand, this fundamental characteristic of formant synthesis leads to unnatural and robot-like synthetic speech. On the other hand, this technique provides the advantage of controlling a large number of speech parameters, some of which with high correlation to emotional-expressive speech making formant synthesis an interesting approach for synthesizing emotional speech.

In the 1990s, three were the main attempts introducing the aspect of emotional speech through formant synthesis namely, the Affect Editor (Cahn, 1990), the HAMLET (Murray and Arnott, 1995) and the VAESS project (Montero et al., 1998). In the first two approaches, emotion-dependent acoustic modelling techniques were implemented in the formant synthesis system, i.e. DECTalk (Klatt, 1990), where in the third attempt, a more general approach was followed using a more global modelling approach of controlling prosodic and voice quality parameters. In a more resent effort, Burkhardt and Sendlmeier (2000), based on the use of perception tests tried to find the optimal values of five acoustic parameters for different emotions without being influenced by prior knowledge from the literature. Consequently, using these five parameters as basis structure of the system, a further attempt was made to enrich the system with proper control of other parameters influencing the emotional charge in the synthetic speech.

In parallel with the earliest steps in the development of emotional speech synthesis through formant synthesis, concatenative synthesis was introduced in the field of neutral speech synthesis. Diphone synthesis was the first representative of concatenative synthesis, where recordings of natural but with flat prosody (i.e. monotonous F0, duration and loudness) speech, is concatenative together in order to synthesize speech. The proper prosody in synthetic speech is achieved at synthesis time through signal processing techniques, modelling mainly the duration and the pitch of the concatenated diphones (i.e. part of speech signal starting from the middle of one phone till the middle of the next phone). The synthetic speech produced by diphone synthesis achieves much higher quality than that of formant synthesis but still is far from human-like speech due to the distortion of the signal caused by the implementation of the signal processing techniques.

Over the previous decade, a lot of studies were made in diphone synthesis of emotional speech applying mainly two different approaches, the copy-synthesis method (Heuft et al., 1996; Montero et al., 1999; Schröder, 1999) and the use of prosody rules (Mozziconacci et al., 1999; Murray et al., 2000; Rank and Pirker, 1998). 
In the former case, the values of duration and F0 were taken from a recorded utterance produced by a human in order to modify the prosody of the diphones and synthesize the same utterance. In the latter approach, a set of rules concerning duration and F0 was created in order to model the prosody of the diphones separately for each emotion to be synthesized. Since voice quality could not be modelled in diphone synthesis a lot of controversial results were presented on whether modelling only duration and F0 of the diphones could produce emotional synthetic speech of reasonable quality.

As mentioned previously, the development of large speech databases led to the introduction of unit-selection corpus-based speech synthesis technique producing the most natural and intelligible synthetic speech in comparison to all the other speech synthesis approaches. In unit-selection synthesis, the appropriate units of recorded speech are selected from the database following some criteria e.g. the context or the prosody of the unit and the distortion of the signal which will be introduced by the concatenation of two contiguous selected units. The production of emotional synthetic speech using unit-selection synthesis has been achieved mainly with two different approaches, using separate emotional speech databases (Iida et al., 2003; Johnson et al., 2002; Lenzo and Black, 2002) or one blended speech database containing speech of different emotions and different degrees of each emotion (e.g. hot, cold, etc.) (Black, 2003; Hofer et al., 2005; Lenzo and Black, 2002). In the former approach, separate speech databases are recorded for each emotion by the same speaker. In order to synthesize a specific emotion units only from the specific database are selected. In the latter approach, one speech database containing speech of various emotions is used and the emotional charge of each unit is used as a selection criterion for the concatenation of the proper units. The main drawback of unit-selection synthesis is that even though the quality of the synthetic speech produced by unit-selection is very high, there is still no flexibility in the manipulation of the synthetic voice characteristics (e.g., speaking style, speech tempo and rhythm, emotions). This makes mandatory the creation of different databases for different speakers and speaking styles, or the need of continuous enriching the blended database with more speaker styles and emotions when one blended database is used instead.

Moreover, over the last years, statistical parametric speech synthesis has dominated by exploiting hidden Markov models (HMM)-based synthesis in order to model spectral envelope, fundamental frequency, and duration (Zen et al., 2009). By adapting the HMMs to the emphasized and non-emphasized regions or to different 
emotionally coloured speech regions, one may synthesize expressive speech due to the flexibility of such schemes in controlling and changing voice characteristics resorting to small speech databases (Yamagishi et al., 2009). This can be accomplished by means of maximum likelihood linear regression (MLLR) transforms (Tamura et al., 2001; Yamagishi et al., 2004). In this direction a lot of effort has been invested in the development of training and adaptation techniques, such as adaptive training or adaptation of an average voice using different speaking styles or emotions (Yamagishi et al., 2002; Yamagishi \& Kobayashi, 2007), or interpolation techniques creating smooth transitions between speaking styles (Tachibana et al., 2005). However, despite the advantage of the flexibility offered by HMMs in statistical parametric synthesis, HMM-based synthesis is characterized by degradation in speech quality in comparison to unit-selection synthesis. The reasons behind this are mainly the vocoding algorithms used, the accuracy of acoustic modelling, and the oversmoothing caused by the HMMs. Till today, in HMM-based synthesis, a lot of attention has been given to these issues, nonetheless much more effort is needed for reaching the level of quality of synthetic speech produced using unit-selection approach.

\subsection{Introduction to prosody modelling in emotional speech}

In phone duration modelling apart from investigating and evaluating different modelling techniques and in order to take better advantage of the effect of prosodic features in human speech analysis, it is essential to investigate not only the attributes of prosody of neutral speech, but also to examine prosodic features in the context of emotional speech. This research can lead to the incorporation of emotional effect on synthesized speech producing expressive synthetic speech. As mentioned earlier, several approaches introducing emotional speech synthesis have been presented over the years, such as formant synthesis, diphone concatenation, unit selection and HMMbased synthesis. In order to use these approaches to synthesize certain emotions or to implement emotional prosody in TTS systems and generate more expressive speech, prosody modelling is employed, implicitly or explicitly, depending on each approach (Jiang et al., 2005; Tesser et al., 2005; Inanoglu and Young, 2009). 
In this direction, the task of segmental duration modelling of emotional speech is essential to be studied in more detail. The phone duration modelling task in the context of emotional speech, together with the analysis of other prosodic features, would contribute both to the enhancement of the quality of emotional synthetic speech and to the achievement of more expressive synthetic speech.

\subsection{Phone duration modelling in emotional speech}

In this study, the performance of ten different phone duration models (PDMs), which have been reported successful on the phone duration modelling task are evaluated. They represent the four categories of machine learning algorithms (decision trees, linear regression, lazy-learning and meta-learning algorithms), mentioned in Chapter 3. A brief outline of these algorithms follows:

(i) Three decision trees were used, namely the $m 5 p$ model tree (Wang and Witten, 1997) and the $m 5 p R$ regression tree (Quinlan, 1992) based on the M5' algorithm (Wang and Witten, 1997) and the regression tree, Reduced Error Pruning trees (REPTrees) (Kääriäinen and Malinen, 2004).

(ii) Two lazy-learning algorithms were used: the $I B K$ (Aha et al., 1991), which uses the k-nearest neighbors algorithm $(k-\mathrm{NN})$, and the Locally Weighted Learning ( $L W L)$ algorithm (Atkeson et al., 1996), which assigns weights using an instance-based method. Concerning the IBK algorithm, for locating the instance that is closer to the training instance, it searches among the $\mathrm{k}$ nearest neighbors of the instance. Evaluating this method with different number of neighbors resulted in the adaptation of 12 neighbors $(k$ $=12$ ), since it gave the best results. In the case of LWL, the kernel function which is used to calculate weights for the data points was the tricube kernel function, while REPTrees were used as classifiers.

(iii) Two meta-learning algorithms were used: the Additive Regression (Add. Reg.) (Friedman, 2002) and the Bagging algorithm (Bagg.) (Breiman, 1996), using two different regression trees (m5pR and REPTrees) as base classifiers. In the two cases of additive regression meta-learning algorithm the shrinkage parameter, $v$, indicating the learning rate, was set equal to 
0.5 and the number of the regression trees, rt-num, was set equal to 10 after grid search experiments $(v=\{0.1,0.3,0.5,0.7,0.9\}, r t-n u m=\{5,10$, $15,20\})$ on a randomly selected subset of the training set, representing the $40 \%$ of the size of the full training set. In the two cases of the bagging algorithm, the number of the regression trees, rt-num, was set equal to 10 after some grid search experiments $(r t-n u m=\{5,10,15,20\})$ on the randomly selected subset of the training set, mentioned earlier.

(iv) Finally, the linear regression (LR) (Witten and Frank, 2005) algorithm was used for our experiments, which is a classification and prediction algorithm that expresses the class variable as a linear combination of the features. The error estimation in LR algorithm is given by the Akaike Information Criterion (AIC) (Akaike, 1974).

\subsubsection{Database}

The positional and contextual factors of a phone (place in syllable, word etc) play a very important role in the assessment of its duration (Febrer et al., 1998; Krishna et al., 2004; Möbius and Santen, 1996; van Santen, 1992). The Modern Greek database, which was used in our experiments, was designed following this statement, so as for each phone to have multiple instances in various positions (initial, medial, final) in different words in the database. The contents of the database were extracted from passages, newspapers or were set up by a professional linguist. The database which was used for the experiments consisted of 62 utterances, which are pronounced several times with different emotional charge. The length of the utterances was ranging from a single word, a phrase, short and long sentence or even a sequence of sentences of fluent speech. The context of all sentences was emotionally neutral, meaning that it did not convey any emotional charge through lexical, syntactic or semantic means. Moreover, all the utterances were uttered separately in the five emotional styles.

The entire database consisted of 4150 words (310 utterances). A phone inventory of 34 phones, with total of 22045 instances (15667 voiced and 6378 unvoiced phones), was used. Furthermore, each vowel class included both stressed and unstressed cases of the corresponding vowel. All utterances were uttered by a 
professional, female actress, speaking Modern Greek. To ensure that the speaker would not have to change her emotional state more than five times, expressing anger, fear, joy, sadness and neutral emotion respectively, all the recordings of each specific emotional category were recorded in series, before proceeding with the other emotional categories. In addition, the actress was instructed to express a 'casual' intensity of the chosen emotional states avoiding any theatrical exaggeration.

All recording sessions were held in the anechoic chamber of a professional studio. Speech was sampled at $44.1 \mathrm{kHz}$, and a resolution of 16 bit. For the needs of our experiments we down-sampled all the recordings to sampling rate of $16 \mathrm{kHz}$.

\subsubsection{Feature set}

In the present study, we consider a number of features which have been reported successful on the task of phone duration modelling (Bell et al., 2003; Chung, 2002; Crystal and House, 1988; Febrer et al., 1998; Goubanova and King, 2008; Gregory et al., 2001; Iwahashi and Sagisaka, 2000; Klatt, 1987; Yamagishi et al., 2008; Krishna and Murthy, 2004; Krishna et al., 2004; Lazaridis et al., 2007; Lee and Oh, 1999b; Möbius and Santen, 1996; Riley, 1992; Takeda et al., 1989; Teixeira and Freitas, 2003; van Santen, 1992; van Santen, 1994). Thus, the feature set is composed of linguistic features extracted only from text - since the input in a speech synthesis system is text. Specifically, from each utterance we computed 33 features. In brief, eight phonetic, three segment-level, thirteen syllable-level, two word-level, one phrase-level and six accentual features were used. Along with these, which concern each current instance, the respective information concerning the one or two previous and next instances (temporal context information) was also used for some of these features.

The overall size of the feature set is 93 (Section 4.1), including the aforementioned features along with their temporal context information as reported above (one or two previous and next instances on the level of the respective feature, phone-level, syllable-level, word-level). 


\subsubsection{Performance evaluation metrics}

The performance of the phone duration prediction models was measured in terms of root mean square error (RMSE), mean absolute error (MAE) and the correlation coefficient (CC). The RMSE is frequently used as a global measure sensitive to gross errors. The MAE, described as the average magnitude of the errors in a set of predictions, does not consider the direction of the deviations from the ground truth and is not that sensitive to gross errors, as the RMSE is. These two figures of metrics are formulated as:

$$
R M S E=\sqrt{\frac{\sum_{i=1}^{N}\left(F\left(x_{i}\right)-y_{i}\right)^{2}}{N}}
$$

and

$$
M A E=\frac{\sum_{i=1}^{N}\left|F\left(x_{i}\right)-y_{i}\right|}{N}
$$

where $N$ is the number of the test instances, $y_{i}$ is the actual duration of $i t h$ instance and $F\left(x_{i}\right)$ is the predicted value for the $i t h$ instance.

Finally, the correlation coefficient (CC) which measures the statistical correlation between the actual and the predicted values of the phone duration, is used. The CC is defined as

$$
C C=\frac{\operatorname{cov}(F(\mathrm{X}), \mathrm{Y})}{s_{F(\mathrm{X})} s_{\mathrm{Y}}}=\frac{\mathrm{E}((F(\mathrm{X})-\bar{F}(\mathrm{X}))(\mathrm{Y}-\overline{\mathrm{Y}}))}{s_{F(\mathrm{X})} s_{\mathrm{Y}}}
$$

where $F(X)$ is the variable of the predicted values and $Y$ are the actual values of the phone durations. The $\bar{F}(x)$ and $\bar{Y}$ are the mean values of the two variables and $S_{F(X)}$ and $S_{Y}$ are the standard deviations of the variables $F(X)$ and $Y$, respectively. Together, these three performance measures offer a good indication about the accuracy of different models. 


\subsubsection{Experimental results}

All the evaluated PDMs, as shown in Table 5.1 and Table 5.2, demonstrated reasonable performance, yielding RMSE values between 19.0 and 30.3 milliseconds, and MAE values between 14.0 and 22.2 milliseconds. The best model, trained with the full set of features, for each category of emotional speech is shown in italics. Despite the fact that these values are bearable for the needs of emotional speech synthesis, a more accurate phone duration model (prediction error $<20 \mathrm{~ms}$ ) would result in synthetic speech of better quality (Wang et al., 2004). Regarding the CC (see Table 5.3), the PDMs achieved values in the range of 0.54 to 0.83 , which is a respectable outcome for a phone duration model. The highest accuracy for phone duration modelling in all categories of emotional speech was observed for the $m 5 p$ trees and the meta-learning, Additive Regression and Bagging, algorithms using $m 5 p R$ regression trees as base classifiers (Add.Reg.m5pR and Bagg.m5pR).

Table 5.1. RMSE values in milliseconds for the different categories of emotional speech.

\begin{tabular}{lccccc}
\hline $\boldsymbol{P D} \boldsymbol{M}$ & Anger & Fear & Joy & Neutral & Sadness \\
\hline Add.Reg.m5pR & 22.1 & 20.1 & 19.0 & 26.3 & 20.6 \\
Add.Reg. REPTrees & 23.8 & 21.3 & 20.8 & 26.7 & 22.1 \\
Bagg.m5pR & 23.3 & 20.9 & 20.4 & 26.7 & 21.4 \\
Bagg. REPTrees & 28.2 & 22.5 & 22.8 & 27.6 & 24.3 \\
\hline IB12 & 24.7 & 21.8 & 22.2 & 27.5 & 20.6 \\
LWL & 28.6 & 24.4 & 23.4 & 28.9 & 25.7 \\
\hline LR & 22.8 & 22.0 & 19.8 & 26.4 & 20.8 \\
\hline m5p & 21.7 & 20.2 & 19.5 & 26.2 & 20.9 \\
m5pR & 24.1 & 21.6 & 21.6 & 27.2 & 22.1 \\
REPTrees & 30.3 & 24.3 & 24.5 & 29.4 & 26.6 \\
\hline
\end{tabular}


Table 5.2. MAE values in milliseconds for the different categories of emotional speech.

\begin{tabular}{lccccc}
\hline PDM & Anger & Fear & Joy & Neutral & Sadness \\
\hline Add.Reg.m5pR & 16.3 & 14.9 & 14.0 & 17.5 & 15.6 \\
Add.Reg. REPTrees & 17.5 & 15.7 & 15.3 & 17.8 & 16.8 \\
Bagg.m5pR & 17.1 & 15.4 & 15.1 & 17.7 & 16.2 \\
Bagg. REPTrees & 20.5 & 16.5 & 16.7 & 18.6 & 18.1 \\
\hline IB12 & 18.0 & 15.8 & 16.4 & 18.4 & 15.6 \\
LWL & 20.5 & 18.0 & 17.0 & 19.3 & 19.0 \\
\hline LR & 17.1 & 16.0 & 14.9 & 17.7 & 16.1 \\
\hline m5p & 16.1 & 15.0 & 14.8 & 17.1 & 16.0 \\
m5pR & 17.6 & 16.0 & 15.9 & 18.2 & 16.8 \\
REPTrees & 22.2 & 18.2 & 17.9 & 20.1 & 20.0 \\
\hline
\end{tabular}

Table 5.3. CC values for the different categories of emotional speech.

\begin{tabular}{lccccc}
\hline PDM & Anger & Fear & Joy & Neutral & Sadness \\
\hline Add.Reg.m5pR & 0.83 & 0.72 & 0.78 & 0.66 & 0.75 \\
Add.Reg. REPTrees & 0.79 & 0.67 & 0.73 & 0.65 & 0.70 \\
Bagg.m5pR & 0.81 & 0.70 & 0.75 & 0.66 & 0.73 \\
Bagg. REPTrees & 0.70 & 0.62 & 0.66 & 0.62 & 0.63 \\
\hline IB12 & 0.78 & 0.66 & 0.69 & 0.63 & 0.75 \\
LWL & 0.70 & 0.55 & 0.65 & 0.59 & 0.59 \\
\hline LR & 0.81 & 0.66 & 0.76 & 0.66 & 0.74 \\
\hline m5p & 0.83 & 0.72 & 0.77 & 0.67 & 0.74 \\
m5pR & 0.79 & 0.66 & 0.70 & 0.63 & 0.70 \\
REPTrees & 0.65 & 0.55 & 0.60 & 0.57 & 0.54 \\
\hline
\end{tabular}

It is also observed that the $L R$ model although showing higher error rates, has performance, which approaches that of the $m 5 p R$ regression trees, showing an average performance drop over all the emotional categories in terms of a relative increase of RMSE and MAE by $3.4 \%$ and $3.5 \%$ in respect to $m 5 p$. Regarding the CC, a respective reduction by $2.7 \%$ was shown. Moreover, it is interesting to point out that between the two local learning methods that were employed, $L W L$ rather than $I B 12$, performed worse in all categories of emotional speech, showing an average performance drop over all the emotional categories in terms of a relative increase of RMSE and MAE by $12.6 \%$ and $11.6 \%$ in respect to $I B 12$. Regarding the $\mathrm{CC}$, a respective reduction by $12.1 \%$ was shown. On the contrary, REPTrees appeared to demonstrate the lowest accuracy among all evaluated methods, both as a single model, and as a base classifier for the cases of Additive Regression and Bagging algorithms (Add.Reg. REPTrees, Bagg. REPTrees). 
The $m 5 p$ trees achieved the best performance due to the fact that these models adopt a greedy algorithm. The greedy algorithm constructs a model tree with a nonfixed structure by using a certain stopping criterion, minimizing the error at each interior node, one node at a time, recursively until the best accuracy is achieved. In this way, the computational cost increases, but very robust models are constructed. Moreover, the meta-learning algorithms, processing meta-data and taking advantage of the information that is produced by other methods (in our experiments the $m 5 p R$ and the REPTrees model) were expected to perform well. However, as we can notice, Additive Regression and Bagging performed better when combined with a robust prediction method such as $M 5 p R$, while they didn't perform that well when the REPTrees were used as base classifier, showing an average performance drop over all the emotional categories in terms of a relative increase of RMSE and MAE by $6.4 \%$ and $6.3 \%$ in the case of Add.Reg. REPTrees models and by $11.5 \%$ and $10.9 \%$ in the case of Bagg. REPTrees models. Regarding the CC, a respective reduction by $5.3 \%$ and $11.4 \%$ respectively for Add.Reg. REPTrees and Bagg. REPTrees models was shown. Regarding the other methods, we noticed that local learning methods or methods that apply a more strict strategy of pruning might perform faster, but they do not yield the highest accuracy.

\subsection{Implementation of feature selection algorithms}

In concept-learning problems, often some of the features which are computed from the data are not really related to the target concept. Feature selection algorithms are employed to overcome this problem by selecting only the most appropriate features in respect to the target concept, limiting the computational cost and improving the concept quality and accuracy of the representation (Kira and Rendell, 1992). Most of the algorithms used in machine learning, are designed - in theory - to be able to learn which are the most appropriate features concerning the target concept each time and discard the rest of them (Witten and Frank, 2005). None the less, in practice, including irrelevant features in a dataset often deteriorates the effectiveness of the algorithms since the latter ones are not always capable of eliminating them (Witten and Frank, 2005). 


\subsubsection{Feature selection algorithms}

In the present section we outline two dissimilar feature selection algorithms, namely the Relief (Kira and Rendell, 1992) and the Correlation-based Feature Selection (CFS) (Hall, 1999), which are employed in the experiments reported in Section 5.4.2.

Kira and Rendell (1992) introduced a statistical feature selection algorithm called Relief that uses instance-based learning to assign a relevance weight to each feature in order to achieve maximal margin. The key idea behind this algorithm is to estimate iteratively the quality of attributes, for all the attributes, according to how well their values distinguish between the instances that are near to each other. In brief, Relief iteratively samples instances randomly across the training data and then for each selected instance finds out the closest neighbouring instances of the same and different classes (Witten and Frank, 2005). The margin of a data instance $j$ used in this method is defined as the distance between the near-hit to the instance $j$ and the nearmiss to the instance $j$, respectively, where near-hit and near-miss denote the nearest samples to instance $j$ with the same and different labels, respectively (Kira and Rendell, 1992). The main shortcoming of the Relief algorithm is its ability to handle only binary class problems. This drawback was overcome by the introduction of ReliefF algorithm by Kononenko (1994), which handles multiple classes. Furthermore the ReliefF algorithm can handle noisy and incomplete data. In the following we consider the RReliefF algorithm which was introduced by Robnik-Sikonja and Kononenko (1997) in order to handle continuous classes in regression problems. Once the use of nearest hits and misses is not feasible in regression problems, the probability that the predicted values of two instances are different is used in order to find the closest neighbouring instances as mention above. Consequently, for each attribute a quality degree is measured according to how well its values distinguish between instances that are near to each other. This algorithm is based on the rational that a useful feature should differentiate between instances with relative large distance between the predicted values of them and have the same value for instances with relative small distance between their predicted values. The advantage of margin-based feature selection methods is based on the consideration of the global information, which leads to optimal weights of features, making these methods efficient even for 
data with dependent features (Gilad-Bachrach et al., 2004). The number of nearest neighbours for attribute estimation was set equal to 15 after grid search experiments $(\mathrm{k}=\{5,10,15 \ldots 100\})$ on a randomly selected subset of the training set, representing the $40 \%$ of the size of the full training set. The RReliefF algorithm is used in combination with a ranking algorithm, in our case the Ranker (Witten and Frank, 2005). Ranker is a search method for ranking individual features according to their individual evaluations.

The Correlation-based Feature Selection (CFS) technique (Hall, 1999), on the other hand, scores and ranks subsets of features, rather than individual features as Relief algorithm does. It uses the criterion that a good feature subset for a classifier contains features that are highly correlated with the class variable but poorly correlated with each other (Hall, 1999). In an iterative scheme the CFS adds features that have the highest correlation with the target, provided that the set does not already contain a feature whose correlation with the target is not higher from the one of the feature in question (Witten and Frank, 2005). Base on this rational the CFS algorithm is capable of dealing not only with irrelevant but also with redundant features. The CFS algorithm overcomes the problem of missing values by replacing them with the mean of all the values in the case of continuous attributes and with the most common value in the case of discrete attributes (Hall, 2000). Furthermore, CFS discretizes internally the continuous features, making the algorithm proper for mixed type of input features. In our experiments the $C F S$ algorithm was used in conjunction with a genetic algorithm (GA) (Goldberg, 1989).

\subsubsection{Experimental results}

It is also interesting to compare the results obtained by the phone duration models with the implementation of the two feature selection algorithms (RReliefF and CFS) to the ones obtained by the PDMs using the full feature set, i.e. with no feature selection $(N o F S)$. In Table 5.4 the number of features in the feature sets produced by the two feature selection methods, RReliefF and $C F S$, for all the emotional categories are shown. Applying feature selection led to a significant reduction of the feature vector size: an average reduction over all the emotional categories of $22 \%$ in the case of RReliefF algorithm and $63 \%$ in the case of CFS algorithm was observed in 
comparison to the size of the full feature vector. As can be seen, the average reduction over all the emotional categories in the case of $C F S$ is much bigger than that in the case of RReliefF, primarily due to the CFS strategy to minimize redundancy among the selected features.

Table 5.4. The number of features selected by the two feature selection algorithms, RReliefF and CFS, for the different categories of emotional speech.

\begin{tabular}{|c|c|c|c|c|c|c|c|c|c|c|}
\hline \multirow{2}{*}{$\begin{array}{l}\text { Emotions } \\
\text { Feature } \\
\text { Selection } \\
\text { Algorithms } \\
\end{array}$} & \multicolumn{2}{|c|}{ Anger } & \multicolumn{2}{|l|}{ Fear } & \multicolumn{2}{|l|}{ Joy } & \multicolumn{2}{|c|}{ Neutral } & \multicolumn{2}{|c|}{ Sadness } \\
\hline & RReliefF & CFS & RReliefF & CFS & ReliefF & CFS & RReliefF & CFS & RReliefF & CFS \\
\hline $\begin{array}{l}\text { Number of } \\
\text { Features }\end{array}$ & 76 & 39 & 77 & 38 & 70 & 27 & 72 & 31 & 68 & 37 \\
\hline
\end{tabular}

The decision to build separate phone duration models for each emotional category rather than one model incorporating all the emotional categories is supported by the results of the feature selection procedure. Even though a degree of uniformity existed in the selected features throughout all the emotional categories, in many cases (among which some phonetic features such as the place of articulation, the manner of production or the lip rounding in the case of $C F S$ algorithm and the last-next accent feature in the case of RReliefF algorithm) different features or temporal context information concerning a feature, (i.e. the information of a feature concerning the previous or next instance) were selected in each emotional category. The features which were selected as relevant (by both feature selection algorithms) in most of the emotional categories were mainly the ToBI accents and boundary tones and the stressed-unstressed syllable features along with their temporal context information concerning the next instance, in the category of the accentual features and the syllable break in the category of the phrase-level features. Furthermore, concerning the syllable-level features, the syllable's onset-coda type and size features along with their temporal context information concerning the next instance and the position type of the syllable feature were selected by both the feature selection algorithms. Finally, the onset-rhyme type belonging to the segment-level features category was selected in most of the emotional categories by both feature selection algorithms.

Furthermore, the RReliefF algorithm except for the above features, which were selected by both the feature selection algorithms, selected additional features as relevant to the concept target of phone duration modelling. Some of these features are 
the last-next accent feature concerning the accentual features and both of the two word-level features, i.e. the part-of-speech and the number of syllables in the word features. Moreover several syllable-level features, additional to the ones mentioned above selected from both algorithms, were selected by the RReliefF algorithm, such as the number of the syllables in the utterance and the number of accented syllables and stressed syllables since the last and to the next phrase break. In addition, all the phonetic features, which most of them were rejected by the $C F S$ as irrelevant features, were selected by the RReliefF algorithm, along with most of their temporal context information concerning mainly the one previous and one next instance. Finally, the name of the previous and the name of the next phone features, which are segmentlevel features, were selected by the RReliefF algorithm.

In Tables 5.5-5.7, the performance of all the models for all the emotional categories for both feature selection (RReliefF and CFS) algorithms, in terms of RMSE, MAE and CC, respectively, are presented. Along with these results with the implementation of the feature selection algorithms in the models, for comparison reason the results presented earlier in Section 5.3.4, are presented again. The values of the RMSE and MAE are in milliseconds. The best NoFS model, trained with the full set of features, i.e. without feature selection (NoFS), for each category of emotional speech is shown in italics. Bold font indicates the cases where the phone duration models with feature selection (RReliefF or CFS) outperform the respective ones without feature selection (NoFS). Finally, the best result for each category of emotional speech, regardless if feature selection was implemented on the models or not, is indicated in the cell with grey background. 
Table 5.5. RMSE values in milliseconds for the different categories of emotional speech. Each phone duration model was evaluated for the full feature set, i.e. without feature selection (NoFS), and with feature selection based either on the RReliefF or the CFS methods.

\begin{tabular}{|c|c|c|c|c|c|c|c|c|c|c|c|c|c|c|c|}
\hline \multirow{2}{*}{$P D M$} & \multicolumn{3}{|c|}{ Anger } & \multicolumn{3}{|c|}{ Fear } & \multicolumn{3}{|c|}{ Joy } & \multicolumn{3}{|c|}{ Neutral } & \multicolumn{3}{|c|}{ Sadness } \\
\hline & NoFS & RReliefF & CFS & NoFS & RReliefF & CFS & NoFS & RReliefF & CFS & NoFS & RReliefF & CFS & NoFS & RReliefF & CFS \\
\hline $1 d d$ Reg.m5pR & 22.1 & 22.4 & 22.7 & 20.1 & 20.0 & 22.4 & 19.0 & 19.3 & 22.2 & 26.3 & 26.1 & 28.1 & 20.6 & 20.8 & 22.0 \\
\hline dd.Reg. REPTree & 23.8 & 23.6 & 23.2 & 21.3 & 21.1 & 22.9 & 20.8 & 20.9 & 23.4 & 26.7 & 26.4 & 29.2 & 22.1 & 21.9 & 22.3 \\
\hline Bagg.m5pR & 3.3 & 23.3 & 23.6 & 20.9 & 20.9 & 22.6 & 20.4 & 20.5 & 22.8 & 26.7 & 26.7 & 28.0 & 21.4 & 1.7 & 22.5 \\
\hline Bagg. REPTrees & 28.2 & 28.0 & 23.4 & 22.5 & 22.5 & 23.5 & 22.8 & 22.9 & 25.5 & 27.6 & 27.1 & 29.3 & 24.3 & 24.2 & 22.7 \\
\hline$I B 12$ & 24.7 & 24.3 & 23.9 & 21.8 & 21.6 & 21.5 & 22.2 & 21.4 & 21.0 & 27.5 & 27.3 & 26.9 & 20.6 & 2.5 & 23.4 \\
\hline$L W L$ & 28.6 & 28.5 & 25.0 & 24.4 & 24.1 & 25.1 & 23.4 & 23.1 & 26.1 & 28.9 & 28.3 & 30.9 & 25.7 & 25.4 & 24.2 \\
\hline$L R$ & 22.8 & 23.3 & 23.8 & 22.0 & 19.9 & 22.1 & 19.8 & 20.2 & 22.7 & 26.4 & 26.8 & 28.6 & 20.8 & 21.2 & 22.4 \\
\hline$n 5 p$ & 21.7 & 22.2 & 21.3 & 20.2 & 19.8 & 22.0 & 19.5 & 19.5 & 21.7 & 26.2 & 26.0 & 27.7 & 20.9 & 20.9 & 20.7 \\
\hline$n 5 p R$ & 24.1 & 23.5 & 24.4 & 21.6 & 21 & 23.2 & 21.6 & 21.4 & 23.4 & 27.2 & 27.0 & 28.7 & 22.1 & 22.3 & 23.2 \\
\hline REPTrees & 30.3 & 30.2 & 25.3 & 24.3 & 24.4 & 23.5 & 24.5 & 24.2 & 27.4 & 29.4 & 29.2 & 31.8 & 26.6 & 26.6 & 24.3 \\
\hline
\end{tabular}


Table 5.6. MAE values in milliseconds for the different categories of emotional speech. Each phone duration model was evaluated for the full feature set, i.e. without feature selection (NoFS), and with feature selection based either on the RReliefF or the CFS methods.

\begin{tabular}{|c|c|c|c|c|c|c|c|c|c|c|c|c|c|c|c|}
\hline \multirow{2}{*}{$P D M$} & \multicolumn{3}{|c|}{ Anger } & \multicolumn{3}{|c|}{ Fear } & \multicolumn{3}{|c|}{ Joy } & \multicolumn{3}{|c|}{ Neutral } & \multicolumn{3}{|c|}{ Sadness } \\
\hline & NoFS & RReliefF & CFS & NoFS & RReliefF & CFS & NoFS & RReliefF & CFS & NoFS & RReliefF & CFS & NoFS & RReliefF & CFS \\
\hline$d d . R$ & 16.3 & 16.3 & 16.7 & 14.9 & 14.9 & 16.9 & 14.0 & 14.3 & 16.7 & 17.5 & 17.1 & 19.4 & 15.6 & 15.8 & 16.9 \\
\hline $4 d d . R \epsilon$ & 17.5 & 17.5 & 17.0 & 15.7 & 15.6 & 17.3 & 15.3 & 15.4 & 17.6 & 17.8 & 17.7 & 19.8 & 16.8 & 6.6 & 17.0 \\
\hline Bagg.m5pR & 17.1 & 17.0 & 17.2 & 15.4 & 15.5 & 17.0 & 15.1 & 15.1 & 17.2 & 17.7 & 7 & 19.1 & 16.2 & .4 & 17.2 \\
\hline Bagg. REPTrees & 20.5 & 20.4 & 16.9 & 16.5 & 16.6 & 17.7 & 16.7 & 16.7 & 19.0 & 18.6 & 18.2 & 20.0 & 18.1 & 18.0 & 17.1 \\
\hline$I B 12$ & 8.0 & 17.5 & 17.4 & 15.8 & 15.6 & 15 & 16.4 & 15.6 & 15.3 & 18.4 & 18.0 & 17.7 & 15.6 & 16.8 & 17.8 \\
\hline$L W L$ & 20.5 & 20.3 & 18.1 & 18.0 & 17.6 & 18.7 & 17.0 & 16.7 & 19.6 & 19.3 & 19.0 & 20.7 & 19.0 & 18.6 & 18.1 \\
\hline$\overline{L R}$ & 17.1 & 17.4 & 17.8 & 16.0 & 14.9 & 16.7 & 14.9 & 15.2 & 17.0 & 17.7 & 17.8 & 19.3 & 16.1 & 16.2 & 17.3 \\
\hline$m 5 p$ & 16.1 & 16.3 & 15.9 & 15.0 & 14.6 & 16.5 & 14.8 & 14.5 & 16.3 & 17.1 & 17.0 & 18.5 & 16.0 & 15.9 & 15.5 \\
\hline$m 5 p R$ & 17.6 & 16.8 & 17.8 & 16.0 & 16.0 & 17.6 & 15.9 & 15 . & 17.6 & 18.2 & 18.1 & 19.7 & 16.8 & 17.0 & 17.7 \\
\hline REPTrees & 22.2 & 22.1 & 18.4 & 18.2 & 18.2 & 17.8 & 17.9 & 17.7 & 20.5 & 20.1 & 19.9 & 21.9 & 20.0 & 20.0 & 18.3 \\
\hline
\end{tabular}


Table 5.7. CC values for the different categories of emotional speech. Each phone duration model was evaluated for the full feature set, i.e. without feature selection (NoFS), and with feature selection based either on the RReliefF or the CFS methods.

\begin{tabular}{|c|c|c|c|c|c|c|c|c|c|c|c|c|c|c|c|}
\hline \multirow{2}{*}{$P D M$} & \multicolumn{3}{|c|}{ Anger } & \multicolumn{3}{|c|}{ Fear } & \multicolumn{3}{|c|}{ Joy } & \multicolumn{3}{|c|}{ Neutral } & \multicolumn{3}{|c|}{ Sadness } \\
\hline & NoFS & RReliefF & CFS & NoFS & RReliefF & CFS & NoFS & RReliefF & CFS & NoFS & RReliefF & CFS & NoFS & RReliefF & CFS \\
\hline Add.Reg. $m 5 p R$ & 0.83 & 0.82 & 0.82 & 0.72 & 0.72 & 0.63 & 0.78 & 0.77 & 0.69 & 0.66 & 0.68 & 0.61 & 0.75 & 0.75 & 0.71 \\
\hline Add.Reg. REPTrees & 0.79 & 0.79 & 0.81 & 0.67 & 0.68 & 0.60 & 0.73 & 0.73 & 0.64 & 0.65 & 0.66 & 0.56 & 0.70 & 0.71 & 0.70 \\
\hline Bagg.m5pR & .81 & 0.81 & 0.80 & 0.70 & 0.70 & 0.63 & 0.75 & 0.74 & 0.66 & 0.66 & 0.66 & 61 & 0.73 & 0.72 & 0.69 \\
\hline Bagg. REPTrees & 0.70 & 0.70 & 0.80 & 0.62 & 0.62 & 0.58 & 0.66 & 0.66 & 0.54 & 0.62 & 0.63 & 0.55 & 0.63 & 0.63 & 0.68 \\
\hline IBI2 & 0.78 & 0.79 & 0.80 & 0.66 & 0.67 & 0.68 & 0.69 & 0.72 & 0.73 & 0.63 & 0.64 & 0.66 & 0.75 & 0.70 & 0.66 \\
\hline$\underline{L W L}$ & 0.70 & 0.70 & 0.77 & 0.55 & 0.57 & 0.52 & 0.65 & 0.68 & 0.54 & 0.59 & 0.61 & 0.51 & 0.59 & 0.61 & 0.64 \\
\hline$L R$ & 0.81 & 0.80 & 0.79 & 0.66 & 0.72 & 0.64 & 0.76 & 0.75 & 0.66 & 0.66 & 0.65 & 0.58 & 0.74 & 0.73 & 0.69 \\
\hline$m 5 p$ & 0.83 & 0.82 & 0.84 & 0.72 & 0.73 & 0.65 & 0.77 & 0.77 & 0.70 & 0.67 & 0.69 & 0.62 & 0.74 & 0.74 & 0.76 \\
\hline$m 5 p R$ & 0.79 & 0.81 & 0.78 & 0.66 & 0.66 & 0.59 & 0.70 & 0.71 & 0.64 & 0.63 & 0.64 & 0.58 & 0.70 & 0.70 & 0.67 \\
\hline REPTrees & 0.65 & 0.65 & 0.76 & 0.55 & 0.55 & 0.66 & 0.60 & 0.62 & 0.46 & 0.57 & 0.57 & 0.46 & 0.54 & 0.54 & 0.63 \\
\hline
\end{tabular}


Apart from Joy emotional category, where the best performance was achieved by the PDM with NoFS (AddReg.m5pR), in all the other categories (Anger, Fear, Neutral and Sadness), one of the two feature selection algorithms led to the lowest RMSE and MAE (please refer to Tables 5.5 and 5.6). Along all the emotional categories the highest performance was achieved with the implementation of no feature selection algorithm for the Joy emotional category. This is justified by the lowest weighted standard deviation of the phone's duration corresponding to this category (please refer to Fig. 5.1). Given that it is a less challenging task, feature selection cannot contribute to the enhancement of the phone duration models in Joy emotional category. The PDMs, implemented by $m 5 p$ algorithm, relying on the $C F S$ had the best performance in the categories Anger and Sadness, presenting a relative reduction of RMSE and MAE by $1.8 \%$ and $1.2 \%$ respectively, in Anger category and by $1 \%$ and $3.1 \%$ respectively, in Sadness category. Regarding the CC (refer to Table 5.7), improvement by $1.2 \%$ and $2.7 \%$ was shown in Anger and Sadness respectively. The PDMs, implemented by $m 5 p$ algorithm, employing the RRelief $F$ algorithm presented the best results in the categories Fear and Neutral, presenting a relative reduction of RMSE and MAE by $2 \%$ and $2.7 \%$ respectively, in Fear category and by $0.8 \%$ and $0.6 \%$ respectively, in Neutral category. Regarding the CC, improvement by $1.4 \%$ and 3\% was shown in Fear and Neutral respectively.

These results indicate that the additional effort to perform feature selection is justified, since it contributes to the improvement of the accuracy of phone duration modelling optimizing their performance. This can be seen in the Tables 5.5, 5.6 and 5.7, where the PDMs using feature selection outperformed the respective PDMs with NoFS even if the particular phone duration models didn't have the best accuracy among the other PDMs in the particular category of emotional speech. 


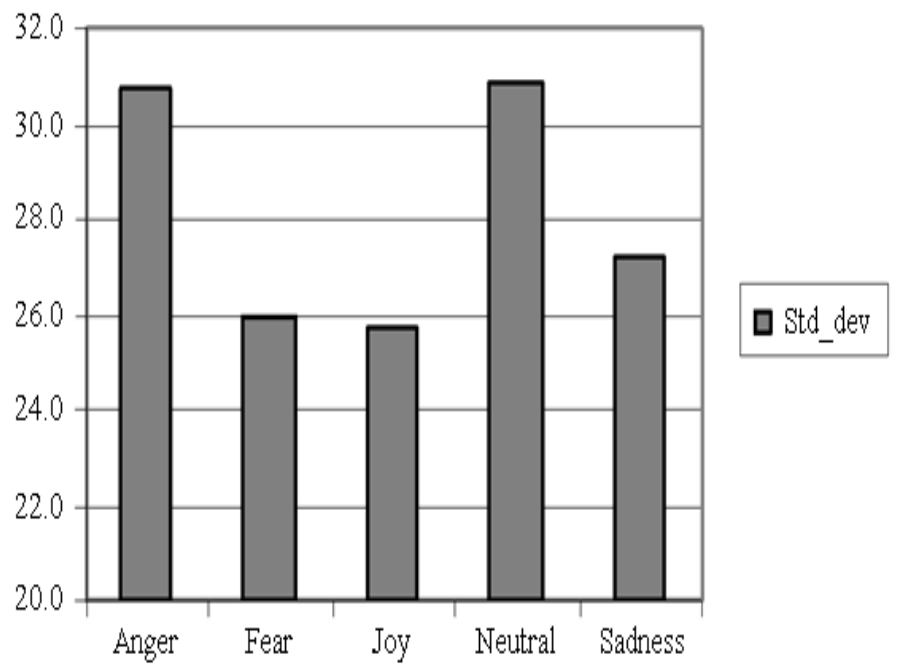

Fig. 5.1. Weighted average values of standard deviations in milliseconds of phone durations for all the emotions.

\subsection{Support vector regression models in emotional speech}

Finally, the support vector regression (SVR) model (Platt, 1999), which implements the sequential minimal optimization (SMO) algorithm for training a support vector classifier (SMOreg) (Smola and Scholkopf, 1998), was used. In our experiments the RBF kernel was used as the mapping function (Scholkopf and Smola, 2002). The basic idea governing the SVR is the production of a model that can be expressed through some support vectors. A linear regression function is used to approximate the training instances by minimizing the prediction error. A userspecified parameter $\varepsilon$ defines a tube around the regression function. In this tube the errors are ignored. The parameter $\varepsilon$ controls how closely the function will fit the training data. The parameter $C$ is the penalty for exceeding the allowed deviation defined by $\varepsilon$. The larger the $C$ is, the more closely the linear regression function can fit the data (Witten and Frank, 2005). The $\varepsilon$ and $C$ parameters, where $\varepsilon \geq 0$ and $C>0$, set equal to $10^{-3}$ and $10^{-1}$ respectively, after a grid search $\left(\varepsilon=\left\{10^{-1}, 10^{-2}, \ldots, 10^{-5}\right\}\right.$, $C=\{0.05,0.1,0.3,0.5,0.7,1.0,10,100\})$ on a randomly selected subset of the training dataset, representing the $40 \%$ of the size of the full training dataset. 


\subsubsection{Experimental results}

In Tables $5.8-5.10$, the results of the SVR models with and without (NoFS) the implementation of the feature selection (RReliefF and CFS) algorithms are presented for RMSE, MAE and CC, respectively. The performance of the best models with no feature selection (NoFS) and with feature selection, reported earlier in Section 5.3.4 and 5.4.2, respectively, are presented again in these tables, for comparison reason. As can be seen, for all the emotional categories, the SVR model with no feature selection (SVR NoFS) outperformed all the other models with and without feature selection. Specifically, in terms of RMSE, SVR NoFS model achieved a relative reduction of $7.51 \%$ in Anger emotional category, $7.58 \%$ in Fear, $6.84 \%$ in Joy, $7.31 \%$ in Neutral and finally, $7.28 \%$ in Sadness emotional category. In terms of MAE, the SVR NoFS model achieved a relative reduction of $9.43 \%$ in Anger emotional category, $6.85 \%$ in Fear, $5.71 \%$ in Joy, $9.41 \%$ in Neutral and finally, 5.81\% in Sadness emotional category. Finally, in terms of CC, the SVR NoFS model achieved a relative increase of $2.83 \%$ in Anger emotional category, 6\% in Fear, $4.21 \%$ in Joy, $5.32 \%$ in Neutral and finally, $3.87 \%$ in Sadness emotional category.

Furthermore, as can be seen in the tables, the SVR model with the implementation of either of the feature selection algorithms (RReliefF and CFS) did not managed to outperform the SVR model without feature selection (SVR NoFS) in none of the emotional categories. The reasoning behind is the high dimensionality of the input feature vector (93 features) in respect to the respectively small amount of data which correspond to each emotional category. The other machine learning techniques do not build robust models from the available training data due to the curse of dimensionality, so the implementation of the feature algorithms helps them deal with the big number of the features. Since the support vector machines do not suffer from this problem they performed better than any of the other techniques and manage to build robust models without the need of the feature selection algorithms to select only the most appropriate features and reduce the size of the feature set. 
Table 5.8. RMSE values in milliseconds for the different categories of emotional speech. For each emotion the best model with (WithFS) and without feature selection (NoFS) and the SVR performance with (RReliefF or CFS) and without (NoFS) feature selection.

\begin{tabular}{|c|c|c|c|c|c|}
\hline RMSE & Anger & Fear & Joy & Neutral & Sadness \\
\hline Best NoFS & $21.7(\mathrm{~m} 5 \mathrm{p})$ & 20.1 (Add. Regg. m5pR) & 19.0 (Add. Regg. m5pR) & $26.2(\mathrm{~m} 5 \mathrm{p})$ & 20.6 (Add. Regg. m5pR) \\
\hline Best WithFS & $21.3(\mathrm{~m} 5 \mathrm{p}-\mathrm{CFS})$ & 19.8 (m5p - RReliefF) & 19.3 (Add. Regg. m5pR - RreliefF) & 26.0 (m5p - RReliefF) & $20.7(\mathrm{~m} 5 \mathrm{p}-\mathrm{CFS})$ \\
\hline SVR NoFS & 19.7 & 18.3 & 17.7 & 24.1 & 19.1 \\
\hline SVR (RReliefF) & 19.9 & 18.6 & 18.1 & 24.4 & 19.5 \\
\hline$S V R(C F S)$ & 21.2 & 20.0 & 18.8 & 25.8 & 20.9 \\
\hline
\end{tabular}

Table 5.9. MAE values in milliseconds for the different categories of emotional speech. For each emotion the best model with (WithFS) and without feature selection (NoFS) and the SVR performance with (RReliefF or CFS) and without (NoFS) feature selection.

\begin{tabular}{|c|c|c|c|c|c|}
\hline$M A E$ & Anger & Fear & Joy & Neutral & Sadness \\
\hline Best NoFS & $16.1(\mathrm{~m} 5 \mathrm{p})$ & 14.9(Add. Regg. m5pR) & 14.0 (Add. Regg. m5pR) & $17.1(\mathrm{~m} 5 \mathrm{p})$ & 15.6 (Add. Regg. m5p) \\
\hline Best WithFS & $15.9(\mathrm{~m} 5 \mathrm{p}-\mathrm{CFS})$ & 14.6 (m5p - RReliefF) & 14.3 (Add. Regg. m5pR - RReliefF) & 17.0 (m5p - RReliefF) & $15.5(\mathrm{~m} 5 \mathrm{p}-\mathrm{CFS})$ \\
\hline SVR NoFS & 14.4 & 13.6 & 13.2 & 15.4 & 14.6 \\
\hline SVR (RReliefF) & 14.6 & 13.7 & 13.5 & 16.1 & 14.8 \\
\hline$S V R(C F S)$ & 15.5 & 14.8 & 14.2 & 16.8 & 15.5 \\
\hline
\end{tabular}


Table 5.10. CC values in milliseconds for the different categories of emotional speech. For each emotion the best model with (WithFS) and without feature selection (NoFS) and the SVR performance with (RReliefF or CFS) and without (NoFS) feature selection.

\begin{tabular}{lccccc}
\hline C C & Anger & Fear & Joy & Neutral & Sadness \\
\hline Best NoFS & 0.83 (m5p) & 0.72 (Add. Regg. m5p) & 0.78 (Add. Regg. m5pR) & 0.67 (m5p) & 0.75 (Add. Regg. m5p) \\
Best WithFS & 0.84 (m5p - CFS) & 0.73 (m5p - RreliefF) & 0.77 (Add. Regg. m5pR - RReliefF) & 0.69 (m5p - RReliefF) & 0.76 (m5p - CFS) \\
SVR NoFS & 0.86 & 0.77 & 0.81 & 0.73 & 0.79 \\
SVR (RReliefF) & 0.86 & 0.76 & 0.79 & 0.70 & 0.78 \\
SVR (CFS) & 0.84 & 0.72 & 0.76 & 0.70 & 0.75 \\
\hline
\end{tabular}




\subsection{Conclusions}

In this chapter, an overview on various phone duration modelling techniques studied over the years and evaluated ten different phone duration models, based on decision trees, linear regression, lazy-learning algorithms and meta-learning algorithms, in the context of emotional speech, was offered. In this evaluation all the features introduced in the literature on the phone and syllable duration prediction tasks were employed. Two feature selection techniques, namely the RReliefF and Correlation-based Feature Selection (CFS) algorithms were involved for performance optimization of the phone duration models. A Modern Greek database of emotional speech was used in all experiments. The experimental results demonstrated that in four out of the five categories of emotional speech, i.e. Anger, Fear, Neutral and Sadness, regardless of the machine learning algorithm used in the phone duration models, feature selection contributed for achieving a better phone duration prediction accuracy, when compared to the case without feature selection. In Joy emotional category, the best model was built with the full feature set, and feature selection didn't bring better accuracy. This might be related to the low value of the weighted standard deviation of Joy emotional category leading to a very robust phone duration model even without the implementation of feature selection. The m5p trees based phone duration model was observed to achieve the best phone duration prediction accuracy in terms of RMSE and MAE among all evaluated models.

Furthermore the SVR algorithm was also evaluated on the phone duration modelling task in emotional speech, outperforming all the other models with and without feature selection in all the emotional categories. Finally, the SVR model with feature selection did not manage to outperform the SVR with no feature selection models in none of the emotional categories. 


\section{Chapter 6}

\section{PERCEPTUAL EVALUATION OF THE PROSODIC MODELS}

Since, phone duration modelling in TTS systems, determines the tempo and rhythm of synthetic speech, and therefore influences the quality and particularly the naturalness of the speech, the need for subjective evaluation becomes clear. Thus, apart from the objective tests based on RMSE, MAE and STD of AE, in this chapter, perceptual tests are performed evaluating the performance of the proposed methods in terms of naturalness of synthetic speech.

\subsection{Experimental setup}

The methodology introduced in (IEEE, 1969) concerning category judgment was implemented in these perceptual tests. Fifteen subjects participated in the evaluation tests, 9 males and 6 females. In the group of the participants both experts and nonexpert in the speech technology were included. The ages of the group ranged from 25 to 45 and all of them were native speakers of both languages, Greek and English. The perceptual test took place in a small room in a free of noise environment using two speakers for the reproduction of speech rather than headphones. One of the most well known and widely used subjective test is the mean opinion score (MOS) (ITU, 2003). 
In this subjective test the quality of every utterance is rated by human listeners according to a 5-point scale, shown in Table 6.1.

Table 6.1. Mean opinion score (MOS) scale for the perceptual evaluation tests.

\begin{tabular}{cc}
\hline Rating & Quality \\
\hline 1 & Bad \\
2 & Poor \\
3 & Fair \\
4 & Good \\
5 & Excellent \\
\hline
\end{tabular}

For each of the databases (Greek - WCL-1 and English - KED TIMIT databases), 10 sentences of the test dataset of length varying from 5 to 15 words were used. Two categories of stimulus were used in our evaluation procedure, unmodified and modified stimuli (Sakurai et al., 2003; Obejobi et al., 2007). The former are natural speech sentences included in the test dataset of the databases with no acoustic modification or any other processing. The latter are modified stimulus based on the phones' durations predicted from the respective model each time for the specific sentence of the test dataset of the databases. In order to produce the modified stimulus the copy-synthesis (Laprie and Bonneau, 2007) method was used. The PRAAT (Boersma and Weenink, 2008) was used in order to modify the phones' durations of the original stimulus according to the predicted phones' durations and Pitch Synchronous Overlap Add (Moulines and Charpentier, 1990) method was used for synthesizing these stimulus.

For each database, for each of the ten sentences, the subjects were presented in random order with one unmodified (original speech-Natural) and 4 modified stimulus, based on the predicted phones' durations of the second-best individual (Second-best) model, and the three proposed models, i.e. the individual SVR model (SMOreg), the SVR-fusion (SMOreg-fusion) scheme and the SVR-FVE (SMOreg-FVE) scheme. The subjects were asked to focus on the timing of the speech. They could listen to each stimulus as many times as they wanted and were asked to score them in a scale of 1-5 according to the MOS scale (ITU, 2003). 


\subsection{Experimental results}

In Table 6.2 the results of the perceptual tests in MOS scale along with the standard deviation of the scores (STD), for the English (KED TIMIT) and Greek (WCL-1) databases are presented. Specifically, in Table 6.2 (a) and 6.2 (b) the results for the KED TIMIT database and for the WCL-1 database are shown, respectively. For comparison reasons along with the results of the perceptual tests, the results of the objective tests, i.e. the MAE and the RMSE, are presented in Table 6.2. As can be seen in both databases, the original speech (Natural) achieved the best scores, 4.75 and 4.82 in MOS scale for the KED TIMIT and the WCL-1 databases respectively, as expected. All the proposed methods, i.e. individual SVR (SMOreg) model, fusion scheme using SVR (SMOreg-fusion) and FVE scheme using SVR (SMOreg-FVE), outperformed the second best individual model for both databases. The SMOreg-FVE models achieved the highest scores 3.72 and 3.87 respectively for KED TIMIT and WCL-1 databases, followed by the SMOreg-fusion models with 3.63 and 3.77 respectively. Furthermore the individual SMOreg models achieved lower scores in comparison to the other two proposed phone duration modelling methods, 3.51 and 3.65 respectively for KED TIMIT and WCL-1 databases, but nonetheless managed to outperform the second-best individual models for both databases. It should be noted that the results of the MOS tests are in line with the results of the objective tests as can be seen in the table.

Table 6.2 (a). Perceptual evaluation tests in MOS scale. MOS score and the standard deviation (STD) of MOS, and the MAE and the RMSE of the respective models forthe KED TIMIT database.

\begin{tabular}{lcccc}
\hline PDMs on the KED TIMIT database & MOS & STD & MAE $(\boldsymbol{m s})$ & $\boldsymbol{R M S E}(\boldsymbol{m s})$ \\
\hline Natural & 4.75 & 0.48 & - & - \\
Second-best & 3.3 & 0.83 & 15.82 & 21.35 \\
SMOreg & 3.51 & 0.81 & 14.95 & 20.56 \\
SMOreg-fusion & 3.63 & 0.71 & 14.66 & 20.14 \\
SMOreg-FVE & 3.72 & 0.75 & 14.36 & 19.75 \\
\hline
\end{tabular}


Table 6.2 (b). Perceptual evaluation tests in MOS scale. MOS score and the standard deviation (STD) of MOS, and the MAE and the RMSE of the respective models forthe WCL-1 database.

\begin{tabular}{lcccc}
\hline PDMs on the WCL-1 database & MOS & STD & MAE (ms) & RMSE (ms) \\
\hline Natural & 4.82 & 0.42 & - & - \\
Second-best & 3.41 & 0.84 & 18.00 & 26.19 \\
SMOreg & 3.65 & 0.74 & 16.78 & 25.21 \\
SMOreg-fusion & 3.77 & 0.68 & 16.35 & 24.76 \\
SMOreg-FVE & 3.87 & 0.74 & 15.97 & 24.04 \\
\hline
\end{tabular}

Table 6.3. Wilcoxon test on the results of the perceptual evaluation test for: (a) the KED TIMIT database, and (b) the WCL-1 database.

(a) results on the KED TIMIT database

\begin{tabular}{|c|c|c|c|c|c|}
\hline PDMs on the KED TIMIT database & Natural & Second-best & SMOreg & SMOreg-fusic & MOreg-FVE \\
\hline Natural & - & $3.86 \mathrm{E}-26$ & $7.06 \mathrm{E}-26$ & $1.87 \mathrm{E}-26$ & $3.16 \mathrm{E}-25$ \\
\hline Second-best & - & - & $2.58 \mathrm{E}-08$ & $1.54 \mathrm{E}-12$ & $1.40 \mathrm{E}-14$ \\
\hline SMOreg & - & - & - & $1.31 \mathrm{E}-05$ & 9.64E-08 \\
\hline SMOreg-fusion & - & - & - & - & 0.0016 \\
\hline SMOreg-FVE & - & - & - & - & - \\
\hline
\end{tabular}

(b) results on the WCL-1 database

\begin{tabular}{lccccc}
\hline PDMs on the WCL-1 database & Natural & Second-best & \multicolumn{2}{c}{ SMOreg } & SMOreg-fusion SMOreg-FVE \\
\hline Natural & - & $9.52 \mathrm{E}-26$ & $2.07 \mathrm{E}-25$ & $2.91 \mathrm{E}-25$ & $1.50 \mathrm{E}-24$ \\
Second-best & - & - & $1.97 \mathrm{E}-09$ & $2.00 \mathrm{E}-13$ & $9.85 \mathrm{E}-17$ \\
SMOreg & - & - & - & $2.21 \mathrm{E}-05$ & $9.22 \mathrm{E}-09$ \\
SMOreg-fusion & - & - & - & - & $5.79 \mathrm{E}-04$ \\
SMOreg-FVE & - & - & - & & - \\
\hline
\end{tabular}

Finally, in order to investigate whether the results of the perceptual tests are statistically significant, we performed the Wilcoxon tests (Wilcoxon, 1945). The results of the Wilcoxon test are presented in Table 6.3 (a) and Table 6.3 (b), respectively for KED TIMIT and WCL-1 databases. As can be seen in the tables, for both databases, all the pairs of stimulus of the models which took part in the perceptual tests are statistical significant with confidence level of more than $95 \%$.

\subsection{Conclusions}

In this chapter the perceptual tests performed evaluating the impact of the proposed phone duration models to synthetic speech were presented. The PRAAT was 
used in order to modify the phones' durations of the original stimulus of speech according to the phones' durations predicted by the proposed models and PSOLA method was used for synthesizing these stimulus. Fifteen subjects evaluated the naturalness of the stimulus based on the MOS scale. The perceptual test for both the databases confirmed the results of the objective tests showing the improvement achieved by the proposed models in the naturalness of synthesized speech. Finally Wilcoxon test was performed, showing that the results of the MOS test are statistical significant for all the proposed methods for both databases. 


\section{Chapter 7}

\section{CONCLUSION}

\subsection{Summary of the Thesis}

In this Thesis, three machine learning techniques for prosody modelling and specifically for phone duration modelling were presented and evaluated.

The first proposed technique introduced in this Thesis was the Support Vector Regression (SVR) approach. SVR had never before been used in the phone duration modelling task. The SVR model was compared to other widely used state-of-the-art models.

The second proposed approach was a SVR-fusion scheme for the fusion of the predictions of different phone duration models, operating in parallel. Specifically, the predictions from a group of dissimilar and independent to each other individual duration models were fed to a machine learning algorithm, which reconciled and fused the outputs of the individual models, yielding more precise phone duration predictions.

Finally the third proposed scheme was a two-stage phone duration modelling scheme using SVR (SVR-FVE), which was built on a number of independent feature constructors (FCs) employed in the first stage, and a phone duration model (PDM), which operated on an extended feature vector in the second stage. The feature vector, which acted as input to the first stage, consisted of numerical and non-numerical linguistic features extracted from text. The extended feature vector was obtained by 
appending the phone duration predictions estimated by the FCs to the initial feature vector.

Furthermore a study concerning phone duration modelling in emotional speech was presented in this Thesis. Feature selection techniques were applied in order to evaluate the features and select the most appropriate ones, studying how feature selection could help improving phone duration modelling accuracy. Two feature selection techniques, the RReliefF and Correlation-based Feature Selection (CFS) algorithms were involved for performance optimization of the phone duration models in emotional speech. Furthermore, the first proposed approach using SVR was introduced in phone duration modelling in emotional speech, outperforming all the other models with and without the implementation the feature selection techniques.

Finally, apart from the objective evaluation of the proposed phone duration modelling schemes, subjective evaluation was performed in order to measure the impact of the proposed schemes to the quality of the synthetic speech and more precise to the naturalness of it. The subjects (human listeners) evaluated the naturalness of the stimulus based on the MOS scale.

\subsection{Conclusions of the Thesis}

The three proposed approaches were evaluated using two databases of different languages, one American-English and one Greek. The proposed approaches were compared to the state-of-the-art models in the phone duration modelling task.

The SVR model outperformed all the other individual models evaluated in this dissertation. Their ability to outperform all the other models is mainly based on their advantage of coping in a better way with high-dimensionality feature spaces in respect to the other models used in phone duration modelling, which makes them appropriate even for the case when the amount of the training data would be small respectively to the number of the feature set used.

The proposed fusion scheme, taking advantage of the observation that different prediction algorithms perform better in different conditions, when implemented with SVR (SVR-fusion), contributed to the improvement of the phone duration prediction 
accuracy over that of the best individual model (SVR). Furthermore the SVR-fusion model managed to reduce the outliers in respect to the best individual model (SVR).

Moreover, the proposed two-stage scheme using individual phone duration models as feature constructors in the first stage and feature vector extension (FVE) in the second stage, implemented with SVR (SVR-FVE), improved the prediction accuracy over the best individual predictor (SVR), and the SVR-fusion scheme and moreover managed to reduce the outliers in respect to the other two proposed schemes (SVR and SVR-fusion). The SVR two-stage scheme confirms in this way their advantage over all the other algorithms of coping well with high-dimensionality feature sets. The improved accuracy of phone duration modelling contributes to a better control of the prosody, and thus quality of synthetic speech.

Furthermore, in the study concerning the phone duration modelling in emotional speech and how feature selection techniques could improve the performance accuracy, the experimental results demonstrated that in four out of the five categories of emotional speech, i.e. Anger, Fear, Neutral and Sadness, regardless of the machine learning algorithm used for phone duration modelling, feature selection contributed to achieving a better phone duration prediction accuracy, when compared to the case without feature selection. Moreover the SVR algorithm was also evaluated on the phone duration modelling task in emotional speech, outperforming all the other models with and without feature selection in all the emotional categories. The SVR model with feature selection did not manage to outperform the SVR with no feature selection models in none of the emotional categories, showing that the SVR model manages to evaluate the features and use them in the best way without the need of feature selection algorithms. This shows that SVR copes well with small amount of data in respect to the feature set.

Finally, perceptual tests were performed evaluating the impact of the proposed phone duration models to synthetic speech. The perceptual test for both the databases confirmed the results of objective tests showing the improvement achieved by the proposed models in the naturalness of synthesized speech. Finally, Wilcoxon test was performed, showing that the results of the MOS tests are statistical significant for all the proposed methods for both databases. 


\subsection{Future work}

As future direction, it would be interesting investigating in depth how more affective and emotional prosody could be implemented in speech synthesis systems, synthesizing affective or emotional speech and furthermore synthesizing conversational speech rather than "reading style" speech. In order to produce this kind of synthetic speech using unit-selection corpus based speech synthesis systems, which is the state-of-the-art method in speech synthesis, large, databases containing speech of the respective emotions or speaking styles, must be recorded each time. Moreover, hidden Markov model (HMM)-based speech synthesis which is also a state-of-the-art method in speech synthesis, allows the development of emotional speech synthesis systems, using adaptation modelling techniques, using small databases of emotional or affecting speech. Still much research effort needs to be invested in this field of speech synthesis mainly for the transition from reading style to conversational speech and from raw emotional to expressive affective synthetic speech. 


\section{LIST OF PUBLICATIONS}

\section{$\underline{\text { Publications in Journals }}$}

[j1] Alexandros Lazaridis, Todor Ganchev, Iosif Mporas, Evaggelos Dermatas, Nikos Fakotakis, "Two-stage phone duration modelling with feature construction and feature vector extension for the needs of speech synthesis", Computer Speech and Language, (Under Review).

[j2] Alexandros Lazaridis, Iosif Mporas, Todor Ganchev, George Kokkinakis, Nikos Fakotakis, "Improving Phone Duration Modeling using Support Vector Regression Fusion", Speech Communication, 53 (1), 85-97, 2011.

[j3] Alexandros Lazaridis, Todor Ganchev, Theodoros Kostoulas, Iosif Mporas, Nikos Fakotakis, "Phone Duration Modeling: Overview of Techniques and Performance Optimization via Feature Selection in the context of Emotional Speech", International Journal of Speech Technology, 13 (3), 175-188, 2010.

[j4] Alexandros Lazaridis, Basiliki Bourna and Nikos Fakotakis, "Comparative Evaluation of Phone Duration Models for Greek Emotional Speech", Journal of Computer Science, 6 (3), 341-349, 2010.

\section{$\underline{\text { Publications in Book Chapters }}$}

[b1] Theodoros Kostoulas, Todor Ganchev, Alexandros Lazaridis, Nikos Fakotakis, "Enhancing Emotion Recognition from Speech through Feature Selection", Text, Speech and Dialogue, TSD 2010, Lecture Notes in Computer Science, Springer Berlin / Heidelberg, pp. 338-344, 2010. 
[b2] Alexandros Lazaridis, Todor Ganchev, Iosif Mporas, Theodoros Kostoulas and Nikos Fakotakis, "Feature Selection for Improved Phone Duration Modeling of Greek Emotional Speech", SETN 2010, Advances in Artificial Intelligence, Lecture Notes in Computer Science, Springer Berlin / Heidelberg, pp. 357-362.

[b3] Todor Ganchev, Alexandros Lazaridis, Iosif Mporas, Nikos Fakotakis, "Performance Evaluation for Voice Conversion Systems", Text, Speech and Dialogue, TSD 2008, Lecture Notes in Computer Science, Springer Berlin/ Heidelberg, pp. 317-324.

[b4] Theodoros Kostoulas, Iosif Mporas, Todor Ganchev, Nikos Katsaounos, Alexandros Lazaridis, Stavros Ntalampiras, Nikos Fakotakis, "LOGOS: A Multimodal Dialogue System for Controlling Smart Appliances", KES IIMSS 2008, New Directions in Intelligent Interactive Multimedia, Studies in Computational Intelligence (142), pp. 585-594.

\section{Publications in Conferences}

[c1] Alexandros Lazaridis, Iosif Mporas, Nikos Fakotakis, "Comparison of HSMM Training Approaches for Emotional Speech Synthesis", ACOUSTICS 2010, Athens, Greece, 4-6 Oct., 2010.

[c2] Alexandros Lazaridis, Theodoros Kostoulas, Todor Ganchev, Iosif Mporas, Nikos Fakotakis, "VERGINA: A modern Greek speech database for speech synthesis", LREC'2010, Malta, 19-21 May, 2010, pp. 117-121.

[c3] Dimitrios P. Lyras, George Kokkinakis, Alexandros Lazaridis, Kyriakos Sgarbas, Nikos Fakotakis "A Large Greek-English Dictionary with Incorporated Speech and Language Processing Tools", ICSLP 2009, 10th Annual Conference of the International Speech Communication Association Brighton, UK, 6-10 Sept. 2009, pp. 1891-1894. 
[c4] Iosif Mporas, Alexandros Lazaridis, Todor Ganchev, Nikos Fakotakis, "Using Hybrid HMM-based Speech Segmentation to improve Synthetic Speech Quality", 13th Pan-Hellenic Conference on Informatics, PCI 2009, Corfu, Greece, 10-12 Sept., 2009, pp. 118-122.

[c5] Vasiliki Bourna, Alexandros Lazaridis, Nikos Fakotakis, "Phone Duration Modeling for Greek Emotional Speech Synthesis", 13th International Conference "SPEECH and COMPUTER", SPECOM 2009, Saint-Petersburg, Russia, 21-25 June, 2009, pp. 190-195.

[c6] Todor Ganchev, Alexandros Lazaridis, Iosif Mporas, Nikos Fakotakis, "Average Performance Loss Measure for Assessment of Voice Conversion Systems", 13th International Conference "SPEECH and COMPUTER", SPECOM 2009, Saint-Petersburg, Russia, 21-25 June, 2009, pp. 275-280.

[c7] Todor Ganchev, Otilia Kocsis, Nikos Katsaounos, Iosif Mporas, Alexandros Lazaridis, Theodoros Kostoulas, Stavros Ntalampiras, Dimitrios Lyras, George Papadopoulos, Kyriakos Sgarbas, Nikos Fakotakis, "Natural Spoken Dialogue Interaction: Technology, Tools, Resources and Applications", System Demonstrations, 18th European Conference on Artificial Intelligence, ECAI 2008, July 2008, pp. 33-34.

[c8] Alexandros Lazaridis, Theodoros Kostoulas, Iosif Mporas, Todor Ganchev, Nikos Katsaounos, Stavros Ntalampiras, Nikos Fakotakis, "Human Evaluation of the LOGOS Spoken Dialogue System", 1st International International Conference on Pervasive Technologies Related to Assistive Environments, PETRA 2008, Athens, Greece, 15-19 July, 2008.

[c9] Alexandros Lazaridis, Panagiotis Zervas, Nikos Fakotakis, George Kokkinakis, "A CART approach for Duration Modeling of Greek Phonemes", 12th International Conference "Speech and Computer", SPECOM 2007, Moscow, Russia, 15-18 Oct., 2007, pp. 287-292. 
[c10] Alexandros Lazaridis, Panagiotis Zervas, George Kokkinakis, "Segmental duration modeling for Greek Speech Synthesis", 19th IEEE International Conference on Tools with Artificial Intelligence (ICTAI), Patras, Greece, 2931 Oct., 2007, pp. 518-521.

\section{$\underline{\text { Technical Reports }}$}

[r1] Alexandros Lazaridis, Nikos Katsaunos, "MoveOn deliverable D5.10.2: Report on the MoveOn TTS component", October 2007.

[r2] Thomas Winkler, Todor Ganchev, Theodoros Kostoulas, Iosif Mporas, Alexandros Lazaridis, Stavros Ntalampiras, Atta Badii, Rick Adderley, Christian Bonkowski, "MoveOn Deliverable D.5: Report on Audio databases, Noise processing environment, ASR and TTS modules", December 2007.

\section{$\underline{\text { Third-partv Citations }}$}

[t1] Alexandros Lazaridis, Panagiotis Zervas, Nikos Fakotakis, George Kokkinakis, "A CART approach for Duration Modeling of Greek Phonemes", 12th International Conference "Speech and Computer", SPECOM 2007, Moscow, Russia, 15-18 Oct., 2007, pp. 287-292.

- Cited in:

- Milan Secujski Darko Pekar, Dragan Knezevic, Vladimir Svrkota, Prosody prediction in speech synthesis based on regression trees, in Proc of. SinFonljA 3, 2010.

[t2] Alexandros Lazaridis, Theodoros Kostoulas, Iosif Mporas, Todor Ganchev, Nikos Katsaounos, Stavros Ntalampiras, Nikos Fakotakis, "Human Evaluation of the LOGOS Spoken Dialogue System", 1st International 
International Conference on Pervasive Technologies Related to Assistive Environments, PETRA 2008, Athens, Greece, 15-19 July, 2008.

Cited in:

- Hannig Hannes, Einsatz von sprach- und gerauschsensitiven Systemen in Smart Home Umgebungen, HASE'10 - HCI Aspects of Smart Environments, pp. 19-28, 2010.

[t3] Alexandros Lazaridis, Panagiotis Zervas, George Kokkinakis, "Segmental duration modeling for Greek Speech Synthesis", 19th IEEE International Conference on Tools with Artificial Intelligence (ICTAI), Patras, Greece, 2931 Oct., 2007, pp. 518-521.

Cited in:

- Mporas, I., 2009. Automatic segmentation of digital speech signals and application to speech synthesis, speech recognition and language recognition. Ph.D. Dissertation.

[t4] Alexandros Lazaridis, Panagiotis Zervas, Nikos Fakotakis, George Kokkinakis, "A CART approach for Duration Modeling of Greek Phonemes", 12th International Conference "Speech and Computer", SPECOM 2007, Moscow, Russia, 15-18 Oct., 2007, pp. 287-292.

Cited in:

- Sovilj-Nikić, S., 2008, Modeling of segmental duration in speech synthesis, In Proceedings of the 16th TELFOR, Beograd, Serbia, November 2008, pp. 703-706.

[t5] Alexandros Lazaridis, Panagiotis Zervas, George Kokkinakis, "Segmental duration modeling for Greek Speech Synthesis", 19th IEEE International Conference on Tools with Artificial Intelligence (ICTAI), Patras, Greece, 2931 Oct., 2007, pp. 518-521.

Cited in:

- Bourna, V., 2008. Segmentation of speech in for recognition and synthesis of emotional speech. Diploma Thesis, University of Patras 


\section{REFERENCES}

Aha, D., Kibler, D., 1991. Instance-based learning algorithms. Machine Learning. 6, 37-66.

Anastasakos, A., Schwartz, R., Suh, H., 1995. Duration modeling in large vocabulary speech recognition. In Proc. of ICASSP-1995, Detroit, USA, pp. 628-631.

Allen, J., Hunnicutt, S., Klatt, D.H., 1987. From text to speech: The MITalk system. Cambridge University Press, Cambridge.

Akaike, H., 1974. A new look at the statistical model identification. IEEE Trans. on Automatic Control. 19 (6), 716-723.

Bartkova, K., Sorin, C., 1987. A model of segmental duration for speech synthesis in French. Speech Communication. 6 (3), 245-260.

Beckman, M.E., Ayers, G.M., 1994. Guidelines for ToBI labelling. The Ohio State University, http://www.ling.ohio-state.edu/research/phonetics/E_ToBI

Bell, A., Jurafsky, D., Fosler-Lussier, E., Girand, C., Gregory, M., Gildea, D., 2003. Effects of disfluencies, predictability, and utterance position on word form variation in English conversation. Journal of the Acoustical Society of America. 113 (2), 1001-1024.

Bellegarda, J.R., Silverman, K.E.A., Lenzo, K., Anderson, V., 2001. Statistical prosody modelling: from corpus design to parameter estimation. IEEE Trans. on Speech and Audio Processing. 9 (1), 52-66.

Benesty, J., Sondhi, M.M., Huang, Y., 2007. Springer Handbook of Speech Processing. Springer-Verlag Berlin Heidelberg.

Black, A.W., 2003. Unit Selection and Emotional Speech. In Proc. of EUROSPEECH-2003, Geneva, Switzerland, pp. 1649-1652.

Black, A.W., 2006. CLUSTERGEN: A statistical parametric synthesizer using trajectory modeling. In Proc. of ICSLP-2006. Pittsburgh, Pennsylvania, USA, pp. 1762-1765.

Black, A.W., Lenzo, K., 2000. Building voices in the Festival speech synthesis system. http://festvox.org/bsv/. 
Black, A.W., Taylor, P., 1997. Automatically clustering similar units for unit selection in speech synthesis. In Proc. of EUROSPEECH-1997, Rhodes, Greece, pp. 601604.

Black, A.W., Zen, H., Tokuda, K., 2007. Statistical parametric speech synthesis. In Proc. of ICASSP-2007, Hawaii, pp. 1229-1232.

Breiman, L., Friedman, J., Olshen, R., Stone, C., 1984. Classification and Regression Trees. Belmont: CAWadsworth International Group.

Breiman, L., 1996. Bagging predictors. Machine Learning. 24 (2), 123-140.

Boersma, P., Weenink, D., 2008. Praat: doing phonetics by computer. http://www.fon.hum.uva.nl/praat/

Bourlard, H., Hermansky, H., Morgan, N., 1996. Towards increasing speech recognition error rates. Speech Communication. 18 (3), 205-231.

Burkhardt, F., Sendlmeier, W.F., 2000. Verification of acoustical correlates of emotional speech using formant synthesis. In Proc. of the ISCA Workshop on Speech and Emotion, Northern Ireland, pp. 151-156.

Cahn, J.E., 1990. The generation of affect in synthesized speech. Journal of the American Voice I/O Society. 8, 1-19.

Campbell, W.N., 1992. Syllable based segment duration. In: Bailly, G., Benoit, C., Sawallis, T.R. (Eds.), Talking Machines: Theories, Models and Designs. Elsevier, Amsterdam, pp. 211-224.

Campbell, N., Black, A.W., 1996. Prosody and the selection of source units for concatenative synthesis. In: van Santen, J., Sproat, R., Olive, J., Hirschberg, J., (Eds.), Progress in speech synthesis. Springer Verlag, pp. 279--282.

Carlson, R., Granstrom, B., 1986. A search for durational rules in real speech database. Phonetica. 43, 140-154.

Chen, S.H., Hwang, S.H., Wang, Y.R., 1998. An RNN-based prosodic information synthesizer for Mandarin text-to-speech. IEEE Trans. on Speech and Audio Processing. 6 (3), 226-239.

Chen, S.H., Lai, W.H., Wang, Y.R., 2003. A new duration modeling approach for Mandarin speech. IEEE Trans. on Speech and Audio Processing. 11 (4), 308-320.

Chung, H., 2002, Duration models and the perceptual evaluation of spoken Korean. In Proc. of Speech Prosody, France, pp. 219-222. 
Clark, J., Yallop, C., 1995. An introduction to phonetics and phonology, second ed. Blackwell, Oxford.

Crystal, T.H., House, A.S., 1988. Segmental durations in connected-speech signals: Current results. Journal of the Acoustical Society of America. 83 (4), 1553-1573.

CSTR, 2001. CSTR US KED TIMIT. University of Edinburgh, http://www.festvox.org/dbs/dbs_kdt.html.

Dash, M., Liu, H., 1997. Feature selection for classification. International Journal of Intelligent Data Analysis. 1, 131-156.

Davis, S.B., Mermelstein, P., 1980. Comparison of parametric representations for monosyllabic word recognition in continuously spoken sentences. IEEE Trans. on Acoustics, Speech and Signal Processing. 28 (4), 357-366.

De Jong, K.A., Spears, W.M., Gordon, D.F., 1992. Using genetic algorithms for concept learning, Machine Learning. 8, 5-32.

Denes, P.B., Pinson, E.N., 1993. The speech chain - the physics and biology of spoken language. W.H.Freeman and Co., New York.

Dixon, N.R., Maxey, H.D., 1968. Terminal analog synthesis of continuous speech using the diphone method of segment assembly. IEEE Trans. Audio and Electroacoustics. 16 (1), 40-50.

Dutoit, T., 1997. An Introduction to Text-To-Speech Synthesis, Kluwer Academic Publishers, Dordrecht.

Edwards, J., Beckman, M.E., 1988. Articulatory timing and the prosodic interpretation of syllable duration. Phonetica. 45, 156-174.

Eide, E., Aaron, A., Bakis, R., Hamza, W., Picheny, M., Pitrelli, J., 2004. A corpusbased approach to $<$ AHEM/> expressive speech synthesis authors. In Proc. of ISCA SSW5, Pittsburgh, USA, pp 79-84.

Epitropakis, G., Tambakas, D., Fakotakis, N., Kokkinakis, G., 1993. Duration modelling for the Greek language. In Proc. of EUROSPEECH-1993, Berlin, Germany, pp. 1995-1998.

Febrer, A., Padrell, J. and Bonafonte, A., 1998. Modeling Phone Duration: Application to Catalan TTS. In Workshop of Speech Synthesis, Australia, pp. 4346.

Ferrer, L., Bratt, H., Gadde, V.R.R., Kajarekar, S.S., Shriberg, E., Sonmez, K., Stolcke, A., Venkataraman, A., 2003. Modeling duration patterns for speaker 
recognition. In Proc. of EUROSPEECH-2003, Geneva, Switzerland, pp. 20172020.

Flach, P.A., Lavrac, N., 2000. The role of feature construction in inductive rule learning. In Proc. of the International Conference on Machine Learning 2000, Workshop on Attribute-value and Relational Learning: Crossing the boundaries, pp. 1-11.

Fotinea, S.L., Tambouratzis, G., 2005. A Methodology for Creating a Segment Inventory for Greek Time Domain Speech Synthesis. International Journal of Speech Technology. 8 (2), 161-172.

Freedman, D., Pisani, R., Purves, R., 2007. Statistics, fourth ed., W.W. Norton \& Company, New York London.

Friedman, J.H., 2001. Greedy function approximation: a gradient boosting machine. Ann. Statist. 29 (5), 1189-1232.

Friedman, J.H., 2002. Stochastic gradient boosting. Comput. Statist. Data Anal.. 38 (4), 367-378.

Fukada, T., Tokuda, K., Kobayashi, T., Imai, S., 1992. An adaptive algorithm for melcepstral analysis of speech. In Proc. of ICASSP-1992, California, USA, pp 137140.

Furui, S., 2000. Digital Speech Processing, Synthesis, and Recognition, second ed., Marcel Dekker, New York.

Gao, B., Qian, Y., Wu, Z., Soong, F.K., 2008. Duration Refinement by Jointly Optimizing State and Longer Unit Likelihood. In Proc. of ICSLP-2008, Brisbane, Australia, pp. 2266-2269.

Gauvain, J., Lee, C.H., 1994. Maximum a posteriori estimation for multivariate Gaussian mixture observations of Markov chains. IEEE Trans. Speech Audio Process. 2 (2), 291-298.

Gilad-Bachrach, R., Navot, A., Tishby, N., 2004. Margin based feature selection theory and algorithms. In: Tadepalli, P., Givan, R., Driessens, K. (Eds.), Proceedings of the 21st International Conference on Machine Learning. Banff, Canada: Morgan-Kaufmann, pp. 43-50.

Goldberg D.E., 1989. Genetic algorithms in search, optimization and machine learning. Boston, USA: Addison-Wesley Longman Publishing. 
Goubanova, O., King, S., 2008. Bayesian networks for phone duration prediction. Speech Communication. 50 (4), 301-311.

Goubanova, O., Taylor, P., 2000. Using Bayesian belief networks for model duration in text-to-speech systems. In Proc. of ICSLP-2000, Beijing, China, pp. 427-430.

Gregory, M., Bell, A., Jurafsky, D., Raymond, W., 2001. Frequency and predictability effects on the duration of content words in conversation. Journal of the Acoustical Society of America. 110 (5), 27-38.

Hall, M.A., 1999. Correlation-based Feature Subset Selection for Machine Learning. $\mathrm{PhD}$ thesis, Department of Computer Science, University of Waikato, Waikato, New Zealand.

Hall, M.A., 2000. Correlation-based Feature Selection for Discrete and Numeric Class Machine Learning. In: Langley, P. (Eds.), Proceedings of the 17th International Conference on Machine Learning. San Francisco, CA: Morgan Kaufmann, pp. 359-366.

Hamraz, S.H., Feyzabadi, S.S., 2005. General-Purpose Learning Machine Using KNearest Neighbors Algorithm, RoboCup. Volume 4020 of Lecture Notes in Computer Science, Springer, pp. 529-536.

Harris, C.M., 1953. A study of the building blocks in speech. Journal of the Acoustic Society of America. 25 (5), 962-969.

Hartigan, J. A., 1975. Clustering Algorithms. Wiley.

Hartigan, J.A., Wong, M.A., 1979. A k-means clustering algorithm, Algorithm AS136, Applied Statistics. 28, 100-108.

Hashem, S., Schmeiser, B., 1995. Improving model accuracy using optimal linear combinations of trained neural networks. IEEE Trans. on Neural Networks. 6 (3), $792-794$.

Hayashi, C., 1952. On the prediction of phenomena from qualitative data and the quantification of qualitative data from the mathematicostatistical point of view. Ann. the Institute of Statistical Mathematics. 3 (2), 69-98.

Heath, D., Kasif, S., Salzberg, S., 1993. Learning oblique decision trees. In Proc. of 13th International Conference on Artificial Intelligence, Chambery, France, pp. 1003-1007. 
Heuft, B., Portele, T., \& Rauth, M., 1996. Emotions in Time Domain Synthesis. In Proc. of ICSLP-1996, Philadelphia, USA, pp 1974-1977.

Hofer, G.O., Richmond, K., Clark, R.A.J., 2005. Informed blending of databases for emotional speech synthesis. In Proc. of ICSLP-2005, Lisbon, Portugal, pp. 501504.

Hu, Y.J., Kibler, D., 1996. Generation of attributes for learning algorithms. In Proc. of 13th International Conference on Machine Learning, San Mateo, CA: Morgan Kaufmann, pp. 806-811.

Huang, X., Acero, A., Hon, H.W., 2001. Spoken Language Processing: a guide to theory, algorithm, and system development. Prentice Hall, Redmond.

Hughes, G.E., 1968. On the mean accuracy of statistical pattern recognizers. IEEE Trans. on Information Theory. 14, 55-63.

Hunt, A.J.; Black, A.W., 1996. Unit selection in a concatenative speech synthesis system using a large speech database. In Proc. ICASSP-1996, Atlanta, Georgia, pp. 373-376.

IEEE, 1969. Recommended practice for speech quality measurements. IEEE Transactions on Audio and Electroacoustics. 17 (3), 225-246.

IIda, A., Campbell, N. Higuchi, F., Yasumura, M., 2003. A Corpus based speech synthesis system with emotion. Speech Communication. 40 (1), 161-187.

Inanoglu, Z. and Young, S., 2009. Data-driven emotion conversion in spoken English. Speech Communication. 51 (3), 268-283.

IPA, 1999. The Handbook of the International Phonetic Association. Cambridge University Press.

ITU-TRecommendation P.800.1, 2003. Mean opinion score (MOS) terminology.

Iwahashi, N., Kaiki, N., Sagisaka, Y., 1993. Speech segment selection for concatenative synthesis based on spectral distortion minimization. IEICE Transaction Fundamentals. E76-A, 1942-1948.

Iwahashi, N., Sagisaka, Y., 2000. Statistical modeling of speech segment duration by constrained tree regression. IEICE Trans. Inform. Systems. E83-D (7), 1550-1559.

Jennequin, N., Gauvain, J.L., 2007. Modeling duration via lattice rescoring. In Proc. of ICASSP-2007, Honolulu, Hawaii, pp. 641-644. 
Jiang, D., Zhang, W., Shen, L., Cai, L., 2005. Prosody Analysis and Modeling for Emotional Speech Synthesis. In Proc. of ICASSP-2005, Philadelphia, PA, USA.

Johnson, W.L., Narayanan, S.S., Whitney, R., Das, R., Bulut, M., LaBore, C., 2002. Limited domain synthesis of expressive military speech for animated characters. In Proc. ICSLP-2002, Denver, CO, pp. 163-166.

Juang, B.H., Rabiner, L.R., Levinson, S.E., Sondhi, M.M., 1985. Recent developments in the application of hidden Markov models to speakerindependent isolated word recognition. In Proc. of ICASSP-1985, Florida, USA, pp. 9-12.

Kääriäinen, M., Malinen, T., 2004. Selective Rademacher penalization and reduced error pruning of decision trees. Journal of Machine Learning Research. 5, 11071126.

Kira, K., Rendell, L.A., 1992. A practical approach to feature selection. In Proc. of 9th International Conference on Machine Learning, San Mateo, CA: Morgan Kaufmann, pp. 249-256.

Kittler, J., Hatef, M., Duin, R.P.W., Matas, J., 1998. On combining classifiers. IEEE Trans. on Pattern Analysis and Machine Intelligence. 20 (3), 226-239.

Klatt, D.H., 1976. Linguistic uses of segmental duration in English: Acoustic and perceptual evidence. Journal of the Acoustic Society of America. 59 (5), 12091221.

Klatt, D.H., 1987. Review of text-to-speech conversion for English. Journal of the Acoustical Society of America. 82 (3), 737-793.

Klatt, D.H., 1990. DecTalk user's manual. Digital Equipment Corporation.

Kohavi, R., John, G.H., 1997. Wrappers for feature subset selection. Artificial Intelligence. 97 (1-2), 273-324.

Kohler, K.J., 1988. Zeistrukturierung in der Sprachsynthese. ITG-Tagung Digitalc Sprachverarbeitung. 6, 165-170.

Koller, D., Sahami, M., 1996. Toward optimal feature selection. In Proc. of 13th International Conference on Machine Learning, Bari, Italy, pp. 129-134.

Kominek, J., Black, A.W., 2003. CMU ARCTIC databases for speech synthesis. CMU-LTI-03-177, Language Technologies Institute, School of Computer Science, Carnegie Mellon University. 
Kominek, J., Black, A.W., 2004. A family-of-models approach to HMM-based segmentation for unit selection speech synthesis. In Proc. of ICSLP-2004, Jeju Island, Korea, pp. 1385-1388.

Kononenko, I., 1994. Estimating attributes: Analysis and extensions of Relief. In: Bergadano, F., De Raedt, L. (Eds.), Proceedings of the European Conference Machine Learning. New York: Springer Verlag, pp. 171-182.

Krishna, N.S., Murthy, H.A., 2004. Duration modeling of Indian languages Hindi and Telugu. In Proc. of the 5th ISCA Speech Synthesis Workshop, Pittsburgh, USA, pp. 197-202.

Krstulovic, S., Latorre, J., Buchholz, S., 2008. Comparing QMT1 and HMMs for the Synthesis of American English Prosody. In Proc. of Speech Prosody 2008, pp. 6770.

Kuncheva, L.I., 2002. A theoretical study on six classifier fusion strategies. IEEE Trans. on Pattern Analysis and Machine Intelligence. 24 (2), 281-286.

Laprie, Y., Bonneau, A., 2007. Construction of perception stimuli with copy synthesis. In Proc. of ICPhS-2007, Sarrebruck, Allemagne, pp. 2189-2192.

Latorre, J., Buchholz, S., Akamine, M., 2010. Usages of an external duration model for HMM-based speech synthesis. In Proc. of Speech Prosody 2010, Chicago, USA.

Laver, J., 1980. The phonetic description of voice quality. Cambridge University Press, Cambridge.

Laver, J., 1994. Principles of Phonetics. Cambridge University Press, Cambridge.

Lazaridis, A., Zervas, P., Kokkinakis, G., 2007. Segmental duration modeling for Greek speech synthesis. In Proc. of IEEE ICTAI-2007, Patras, Greece, pp. 518521.

Lazaridis, A., Mporas, I., Ganchev, T., Kokkinakis, G., Fakotakis, N., 2011. Improving Phone Duration Modeling using Support Vector Regression Fusion. Speech Communication. 53 (1), 85-97.

Lee, S., Oh, Y.H., 1999a. Tree-based modeling of prosodic phrasing and segmental duration for Korean tts systems. Speech Communication. 28 (4), 283-300.

Lee, S., Oh, Y.H., 1999b. CART-based modelling of Korean segmental duration. In Proc. of Oriental COCOSDA-1999, Taipei, Taiwan, pp. 109-112. 
Lenzo, K., Black, A.W., 2002. Customized synthesis: blending and tiering. AVIOS2002, San Jose, CA.

Levinson, S., 1986. Continuously variable duration hidden Markov models for speech analysis. In Proc. of ICASSP-1986, Tokyo, Japan, pp.1241-1244.

Ling, Z.H., Wang, R.H., 2006. HMM-based unit selection using frame sized speech segments. In Proc. of ICSLP-2006. Pittsburgh, Pennsylvania, USA, pp. 20342037.

Liu, Z., Yan, J., Zhang, D., Li, Q.L., 2007. Automated tongue segmentation in hyperspectral images for medicine. Applied Optics. 46, 8328-8334.

Matheus, C.J., Rendell, L., 1989. Constructive induction in decision trees. In Proc. of 11 th IJCAI, Detroit, USA, pp. 645-650.

Mitchell, TM., 1997. Machine Learning, McGraw-Hill: Singapore.

Mitchell, C., Harper, M., Jamieson, L., Helzermam, R., 1995. A parallel implementation of a hidden Markov model with duration modeling for speech recognition. Digital Signal Processing. 5, 43-57.

Möbius, B., 2000. Corpus-based speech synthesis: Methods and Challenges. Technical Report, University of Stuttgart, AIMS 6 (4).

Möbius, B., van Santen, J., 1996. Modeling segmental duration in German text-toSpeech synthesis. In Proc. of ICSLP-1996, Philadelphia, USA pp. 2395-2398.

Monkowski, M.D., Picheny, M.A., Rao, P.S., 1995. Context dependent phonetic duration models for decoding conversational speech. In Proc. of ICASSP-1995, Detroit, Michigan, USA, pp. 528-531.

Montero, J.M., Gutierrez-Arriola, J., Colas, J., Enriquez, E., Pardo, J.M., 1999. Analysis and Modelling of Emotional Speech in Spanish. In Proc. of ICPhS-1999, San Francisco, USA, pp. 957-960.

Montero, J.M., Gutierrez-Arriola, J., Palazuelos, S., Enriquez, E., Aguilera, S., Pardo, J.M., 1998. Emotional Speech Synthesis: From Speech Database to TTS. In Proc. of ICSLP-1998, Sydney, Australia, pp. 923-926.

Moulines, E., Charpentier, F., 1990. Pitch-synchronous waveform processing techniques for text-to-speech synthesis using diphones. Speech Communication. 9 (5-6), 453-467. 
Mozziconacci, S.J.L., Hermes, D.J., 2004. Role of intonation patterns in conveying emotion in speech. In Proc. of ICPhS-1999, San Francisco, USA, pp. 2001-2004.

Mporas, I., 2009. Automatic segmentation of digital speech signals and application to speech synthesis, speech recognition and language recognition. PhD Thesis.

Mporas, I., Ganchev, T., Fakotakis, N., 2008. A hybrid architecture for automatic segmentation of speech waveforms. In Proc. of ICASSP-2008. Nevada, USA, pp. 4457-4460.

Mporas, I., Ganchev, T., Fakotakis, N., 2010. Speech segmentation using regression fusion of boundary predictions. Computer Speech and Language. 24 (2), 273-288.

Murray, I.R., Arnott, J.L., 1995. Implementation and testing of a system for producing emotion-by-rule in synthetic speech. Speech Communication. 16 (4), 369-390.

Murray, I.R., Edgington, M.D., Campion, D., Lynn, J., 2000. Rule-based Emotion Synthesis Using Concatenated Speech. In Proc. of ISCA Workshop on Speech \& Emotion, Northern Ireland 2000, pp. 173-177.

Nakano, Y., Tachibana, M., Yamagishi, J., Kobayashi, T., 2006. Constrained structural maximum a posteriori linear regression for average-voice-based speech synthesis. In Proc. of ICSLP-2006, Pittsburgh, USA, pp 2286-2289.

Odejobi, O.A., Wong, S.S., Beaumont, A.J., 2007. A fuzzy decision tree-based duration model for Standard Yoruba text-to-speech synthesis. Computer Speech and Language. 21 (2), 325-349.

Ogata, K., Tachibana, M., Yamagishi, J., Kobayashi, T., 2006. Acoustic model training based on linear transformation and MAP modification for HSMM-based speech synthesis. In Proc. of ICSLP-2006, Pittsburgh, USA, pp 1328-1331.

Olive, J.P., Liberman, M.Y., 1985. Text to speech - an overview. Journal of the Acoustical Society of America. 78 (Suppl. 1):S6.

Pagallo, G., Haussler, D., 1990. Boolean feature discovery in empirical learning. In Proc. of 7 th International Conference on Machine Learning, San Mateo, CA: Morgan Kaufmann, pp. 71-99.

Park, J., Sandberg, I.W., 1993. Approximation and radial-basis-function networks. Neural Computation. 5 (2), 305-316. 
Piramuthu, S., Sikora, R.T., 2009. Iterative feature construction for improving inductive learning algorithms, Expert Systems with Applications. 36 (2), 34013406.

Platt, J., 1999. Fast training of support vector machines using sequential minimal optimization. In: Scholkopf, B., Burges, C., Smola, A. (Eds.), Advances in kernel methods: Support vector learning. MIT Press, Cambridge, pp. 185-208.

Pols, L.C.W., Wang, X., ten Bosch, L.F.M., 1996. Modelling of phone duration (using the TIMIT database) and its potential benefit for ASR. Speech Communication. 19 (2), 161-176.

Pylkkonen, J., Kurimo, M., 2004. Duration modeling techniques for continuous speech recognition. In Proc. of ICSLP-2004, Jeju Island, Korea, pp. 385-388.

Quinlan, R.J., 1992. Learning with continuous classes. In Proc. of 5th Australian Joint Conference on Artificial Intelligence, Singapore, pp. 343-348.

Quinlan, J.R., 1993, C4.5. Programs for Machine Learning, Morgan Kaufman.

Ragavan, H., Rendell, L.A., Shaw, M., Tessmer, A., 1993. Complex concept acquisition through directed search and feature caching. In Proc. of 13th International Conference on Artificial Intelligence, Chambery, France, pp. 946951.

Rank, E., Pirker, H., 1998. Generating Emotional Speech with a Concatenative Synthesizer. In Proc. of ICSLP-1998, Sydney, Australia, pp. 671-674.

Rao, K.S., Yegnanarayana, B., 2005. Modeling syllable duration in Indian languages using support vector machines. In Proc. of ICISIP-2005, India, pp. 258-263.

Rao, K.S., Yegnanarayana, B., 2007. Modeling durations of syllables using neural networks. Computer Speech \& Language. 21(2), 282-295.

Riley, M., 1992. Tree-based modelling for speech synthesis. In: Bailly, G., Benoit, C., Sawallis, T.R. (Eds.), Talking Machines: Theories, Models and Designs. Elsevier, Amsterdam, pp. 265-273.

Robnik-Sikonja, M., Kononenko, I., 1997. An adaptation of relief for attribute estimation in regression. In: Fisher, D.H. (Eds.), Proceedings of the 14th International Conference on Machine Learning. San Francisco, CA: Morgan Kaufmann, pp. 296-304. 
Russel, M.J., R.K. Moore, 1985. Explicit modeling of state occupancy in hidden Markov models for automatic speech recognition. In Proc. ICASSP-1985, Florida, USA, pp. 5-8.

Sakurai, A., Hirose, K., Minematsu, N., 2003. Data-driven generation of f0 contours using a superpositional model. Speech Communication. 40 (4), 535-549.

Schröder, M., 1999. Can emotions be synthesized without controlling voice quality?. Phonus 4, Research Report of the Institute of Phonetics, University of the Saarland, pp. 37-55. http://www.dfki.de/ schroed.

Schröder, M., 2009. Expressive Speech Synthesis: Past, Present, and Possible Futures, in: Tao, J., Tan, T., (Eds.). Affective Information Processing. Springer, London.

Scholkopf, B., Smola, A.J., 2002. Learning with Kernels. MIT Press, Cambridge.

Schweitzer, A., Möbius, B., 2004. Exemplar-based production of prosody: Evidence from segment and syllable durations, In Proc. of Speech Prosody 2004, Nara, Japan, pp. 459-462

Sears A., Jacko, J.A., 2007. Handbook for Human Computer Interaction (2nd ed), CRC Press.

Shannon, R.V., Zeng, F.G., Kamath, V., Wygonski, J., Ekelid, M., 1995. Speech recognition with primarily temporal cues. Science. 13 (5234), 303-304.

Shih, C., Ao, B., 1997. Duration study for the Bell Laboratories Mandarin text-tospeech system. In: van Santen, J., Sproat, R., Olive, J., Hirschberg, J. (Eds.), Progress in Speech Synthesis. Springer, New York, pp. 383-399.

Silén, H., Helander, E., Nurminen, J., Gabbouj, M., 2010. Analysis of Duration Prediction Accuracy in HMM-Based Speech Synthesis. In Proc. of Speech Prosody, Chicago, USA.

Silverman, K., Beckman, M., Pitrelli, J., Ostendorf, M., Wightman, C., Price, P., Pierrehumbert, J., Hirschberg, J., 1992. ToBI: A standard for labeling English Prosody. In Proc. of ICSLP-1992, Banff, Alberta, Canada, pp. 867-870.

Simoes, A.R.M., 1990. Predicting sound segment duration in connected speech: An acoustical study of Brazilian Portugese. In Proc. of Workshop on Speech Synthesis, Autrans, France, pp.173-176.

Smola, A.J., Scholkopf, B., 1998. A tutorial on support vector regression. Royal Holloway College, London, U.K., NeuroCOLT Tech. Rep. TR 1998-030. 
Strom, V., Clark, R., King, S., 2006. Expressive prosody for unit-selection speech synthesis. In Proc. ICSLP-2006, Pittsburgh, USA.

Tachibana, M., Yamagishi, J., Masuko, T., Kobayashi, T., 2005. Speech synthesis with various emotional expressions and speaking styles by style interpolation and morphing. IEICE Trans. Inf. Systems. E 88-D (11), 2484-2491.

Takeda, K., Sagisaka, Y., Kuwabara, H., 1989. On sentence-level factors governing segmental duration in Japanese. Journal of Acoustic Society of America. 86 (6), 2081-2087.

Tamura, M., Masuko, T., Tokuda, K., Kobayashi, T., 2001. Adaptation of pitch and spectrum for HMM-based speech synthesis using MLLR. In Proc. of ICASSP2001, Utah, USA, pp 805-808.

Taylor, P., 2009. Text-to-Speech Synthesis, Cambridge University Press.

Teixeira, J.P., 2004. A Prosody Model to TTS Systems. Phd Thesis, University of Port.

Teixeira, J.P., Freitas, D., 2003. Segmental durations predicted with a neural network. In Proc. of the European Conference on Speech Communication and Technology, Geneva, Switzerland, September, pp. 169-172.

Tesser, F., Cosi, P., Drioli, C. and Tisato, G., 2005. Emotional Festival-Mbrola TTS Synthesis. In Proc. of ICSLP-2005, Lisboa, Portugal, pp. 505-508.

Tokuda, K., Masuko, T., Miyazaki, N., Kobayashi, T., 1999. Hidden markov models based on multi-space probability distribution for pitch pattern modelling. In Proc. of ICASSP-1999, Arizona, USA, pp 229-232.

Tokuda, K., Yoshimura, T., Masuko, T., Kobayashi, T., Kitamura, T., 2000. Speech parameter generation algorithm for HMM-based speech synthesis. In Proc. of ICASSP-2000, Istanbul, Turkey, pp 1315-1318.

Tsiakoulis, P., Chalamandaris, A., Karabetsos, S., Raptis, S., 2008. A statistical method for database reduction for embedded unit selection speech synthesis. In Proc. of ICASSP-2008. Nevada, USA, pp. 4601-4604.

van Santen, J.P.H., 1992. Contextual effects on vowel durations. Speech Communication. 11 (6), 513-546.

van Santen, J.P.H., 1994. Assignment of segmental duration in text-to-speech synthesis. Computer Speech \& Language. 8 (2), 95-128. 
van Santen, J.P.H., 1998. Timing. In: Sproat, R. (Eds.), Multilingual Text-to- Speech Synthesis - The Bell Labs Approach. Springer, New York, pp. 115-139.

van Santen, J., Olive, J., 1990. The analysis of contextual effects on segmental duration. Computer. Speech \& Language. 4 (4), 359-390.

van Santen, J.P.H., Sproat, R.W., Olive, J.P., Hirschberg, J., 1997. Progress in Speech Synthesis. Springer.

Vapnik, V., 1995. The Nature of Statistical Learning Theory. Springer, New York.

Vapnik, V., 1998. Statistical Learning Theory. Wiley, New York.

Vilalta, R., Drissi, Y., 2002. A perspective view and survey of meta-learning. Artificial Intelligence Review. 18 (2), 77-95.

W3C, 1999. W3C, Standard Generalized Markup Language: http://www.w3.org/MarkUp/SGML/

W3C, 2003. W3C, Speech Synthesis Markup Language Version 1.0: http://www.w3.org/TR/2003/CR-speech-synthesis-20031218/

Wang, Y., Witten, I.H., 1997. Inducing model trees for continuous classes. In Poster papers of the 9th European Conference on Machine Learning, Prague, Czech Republic, pp. 128-137.

Wang, L., Zhao, Y., Chu, M., Zhou, J., Cao, Z., 2004. Refining segmental boundaries for TTS database using fine contextual-dependent boundary models. In Proc. of ICASSP-2004, Montreal, Quebec, Canada, pp. 641-644.

Watanabe, L., Rendell, L.A., 1991. Feature construction in structural decision trees, in Proc. of 8th International Conference on Machine Learning, San Mateo, CA: Morgan Kaufmann, pp. 218-222.

Wells, J.C., 1997. SAMPA computer readable phonetic alphabet. In: Gibbon, D., Moore, R., Winski, R., (Eds.), Handbook of Standards and Resources for Spoken Language Systems. Berlin and New York: Mouton de Gruyter. Part IV, section B.

Wenk, J., Michalski, R.S., 1994. Hypothesis-driven constructive induction in AQ17HCI: A method and experiments, Machine Learning. 14, 139-168.

Wilcoxon, F., 1945. Individual comparisons by ranking methods. Biometrics. 1, 8083.

Witten, H.I., Frank, E., 2005. Data Mining: Practical Machine Learning Tools and Techniques, second ed., Morgan Kauffman Publishing, San Francisco. 
Wolpert, D.H., 1992. Stacked generalization, Neural Networks. 5 (2), 241-260.

Wu, Y.J., Kawai, H., Ni, J., Wang, R.H., 2005. Discriminative training and explicit duration modeling for HMM-based automatic segmentation. Speech Communication. 47 (4), 397-410.

Yamagishi, J., Kawai, H., Kobayashi, T., 2008. Phone duration modeling using gradient tree boosting. Speech Communication. 50 (5), 405-415.

Yamagishi, J., Kobayashi, T., 2007. Average-voice-based speech synthesis using HSMM-based speaker adaptation and adaptive training. IEICE Trans. Inf. \& Syst., E90-D (2), 533-543.

Yamagishi, J., Masuko, T., Kobayashi, T., 2004. MLLR adaptation for hidden semiMarkov model based speech synthesis. In ICSLP-2004, Jeju Island, Korea, pp 1213-1216.

Yamagishi, J., Nose, T., Zen, H., Zhen-Hua, L., Toda, T., Tokuda, K., King, S., Renals, S., 2009. Robust Speaker-Adaptive HMM-Based Text-to-Speech Synthesis. IEEE Trans. Audio, Speech and Language Processing. 17 (6), 12081230.

Yamagishi, J., Tamura, M., Masuko, T., Tokuda, K., Kobayashi, T., 2002. A context clustering technique for average voice model in HMM-based speech synthesis. In Proc. of ICSLP-2002, Colorado, USA, pp. 133-136.

Yegnanarayana B., 1999. Artificial Neural Networks. New Delhi, India: Printice-Hall. Yiourgalis, N., Kokkinakis, G., 1996. A TtS system for the Greek language based on concatenation of formant coded segments. Speech Communication. 19 (1), 21-39.

Yoshimura, T., Tokuda, K., Masuko, T., Kobayashi, T., Kitamura, T., 1998. Duration Modeling in HMM-based Speech Synthesis System. In Proc. of ICSLP-1998, Sydney, Australia, pp.29-32.

Yoshimura, T., Tokuda, K., Masuko, T., Kobayashi, T., Kitamura, T., 1999. Simultaneous modeling of spectrum, pitch and duration in HMM-based speech synthesis. In Proc. of EUROSPEECH-1999, Budapest, Hungary, pp 2347-2350.

Young, S., Evermann, G., Gales, M., Hain, T., Kershaw, D., Liu, X., Moore, G., Odell, J., Ollason, D., Povey, D., Valtchev, V., Woodland, P., 2006. The HTK Book (for HTK Version 3.4). Cambridge University Engineering Department. 
Zen, H., Masuko, T., Yoshimura, T., Tokuda, K., Kobayashi, T., Kitamura, T., 2007a. State duration modeling for HMM-based speech synthesis. IEICE Trans. on Inf. \& Syst. E90-D (3), 692-693.

Zen, H., Toda, T., Nakamura, M., Tokuda, T., 2007b. Details of the Nitech HMMbased speech synthesis system for the Blizzard Challenge 2005. IEICE Trans. Inf. Syst. E90-D (1), 325-333.

Zen, H., Nose, T., Yamagishi, J., Sako, S., Masuko, T., Black, A.W., Tokuda, K., 2007c. The HMM-based speech synthesis system (HTS) version 2.0. In Proc. of 6th ISCA Workshop on Speech Synthesis (SSW-6), August 2007.

Zen, H., Tokuda, K., Black A.W., 2009. Statistical parametric speech synthesis, Speech Communication. 51 (11), 1039-1054.

Zen, H., Tokuda, K., Masuko, T., Kobayashi, T., Kitamura, T., 2004. Hidden SemiMarkov Model Based Speech Synthesis. In Proc. of ICSLP-2004, Jeju Island, Korea, pp. 1393-1396.

Zervas, P., Fakotakis, N., Kokkinakis, G., 2008. Development and evaluation of a prosodic database for Greek speech synthesis and research. Journal of Quantitative Linguistics. 15 (2), 154-184.

Zheng, Z., 1996. Constructing nominal x-of-n attributes. In Proc. of 13th International Conference on Machine Learning, San Mateo, CA: Morgan Kaufmann, pp. 10641070. 


\title{
EXTENDED SUMMARY OF THE PHD DISSERTATION IN GREEK LANGUAGE
}

\author{
EKTENH $\Sigma$ ПEPIAHYH \\ TH $\Sigma \Delta$ I $\Delta$ AKTOPIKH $\Sigma \Delta$ IATPIBH $\Sigma$ \\ $\Sigma T H N$ E $\Lambda \Lambda H N I K H ~ Г \Lambda \Omega \Sigma \Sigma A$
}




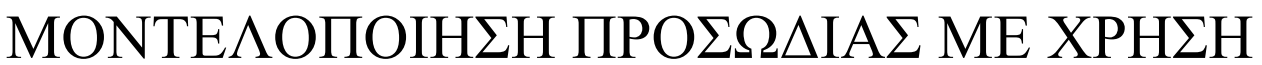 TEXNIK $\Omega$ N MHXANIKH $\Sigma$ MA $\Theta H \Sigma H \Sigma \Sigma T A$ $\Pi \Lambda$ AI $\Sigma$ IA OY $\Delta$ ETEPH $\Sigma$ KAI $\Sigma$ YNAI $\Sigma \Theta H M A T I K H \Sigma$ $\Sigma$ YN $\Theta E T I K H \Sigma$ OMI $\Lambda$ IA $\Sigma$
}

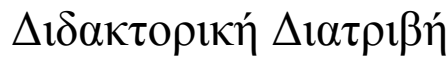 \\ $\tau \mathrm{OU}$
}

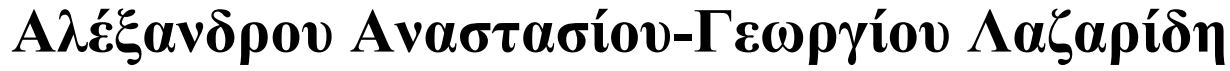

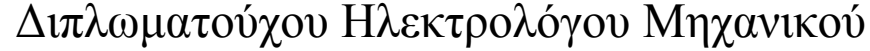

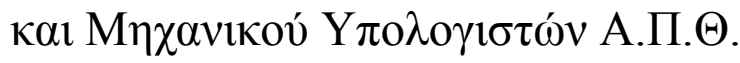

\author{
ПАNЕПI $\Sigma$ THMIO ПАТP $\Omega N$ \\ ПO $\Lambda$ YTEXNIKH $\Sigma$ XO $\Lambda \mathrm{H}$

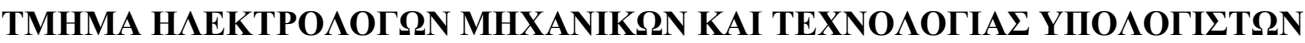

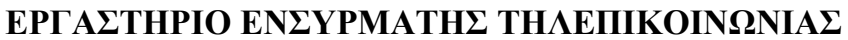

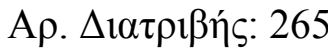

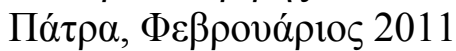




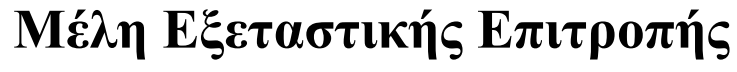

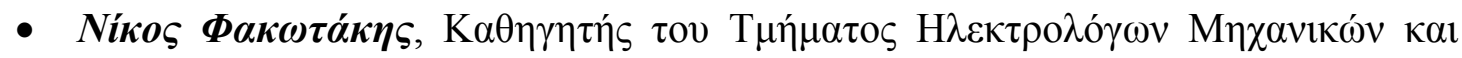

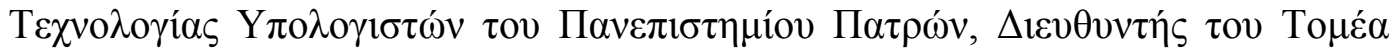

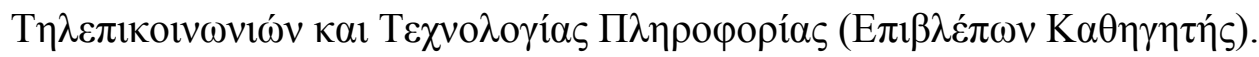

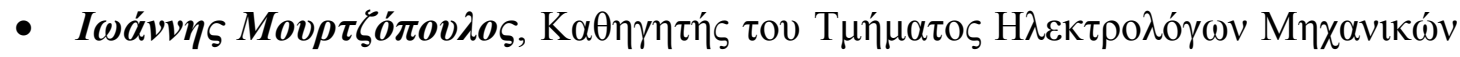

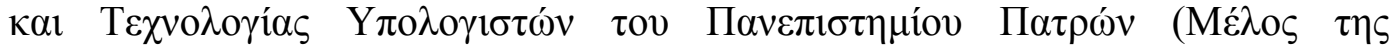

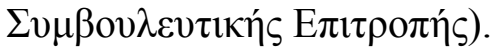

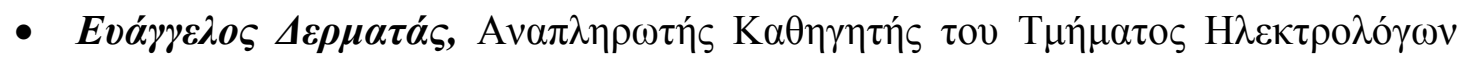

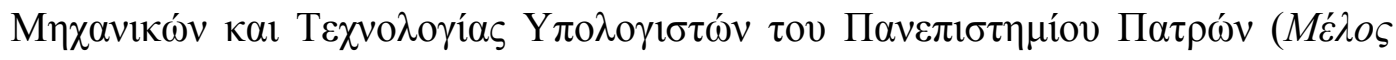

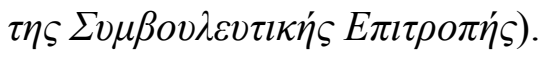

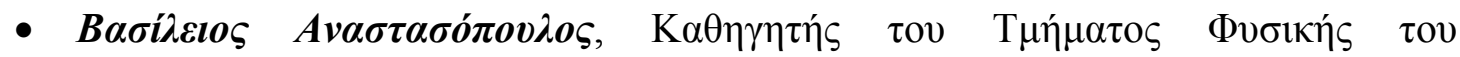

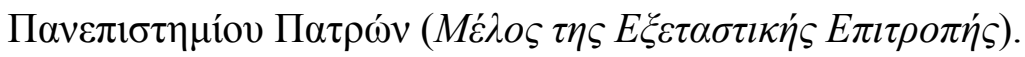

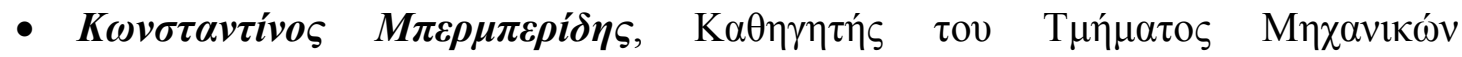

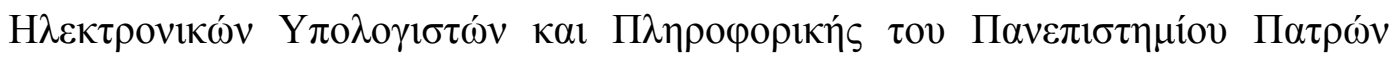

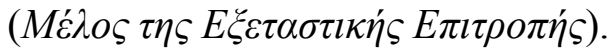

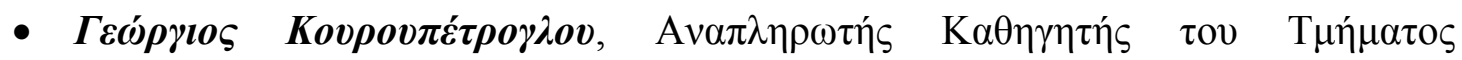

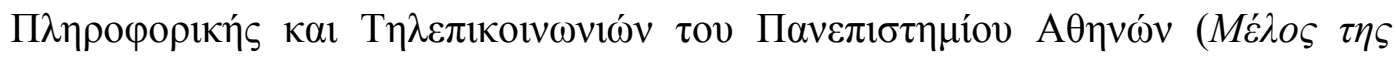
$E \xi \varepsilon \tau \alpha \sigma \tau \imath \kappa \eta ่ \varsigma ~ E \pi \imath \tau \rho o \pi \dot{\varphi} \varsigma)$.

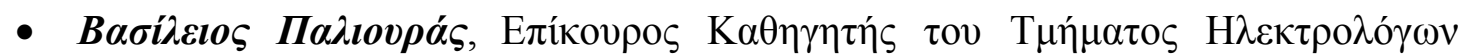

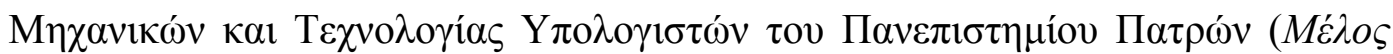

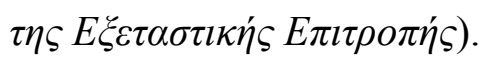




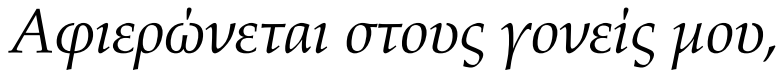

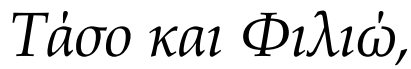
каı ота абе́ $\varphi \imath a$ ноv, Niко кал Mìंбо. 


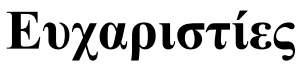

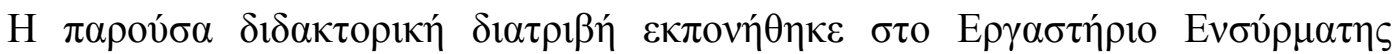

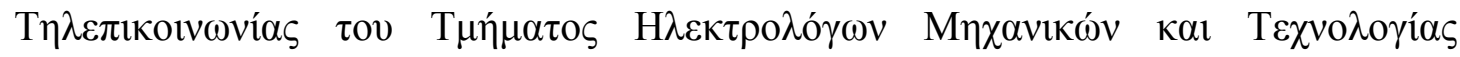

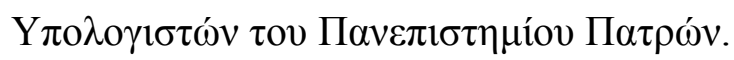

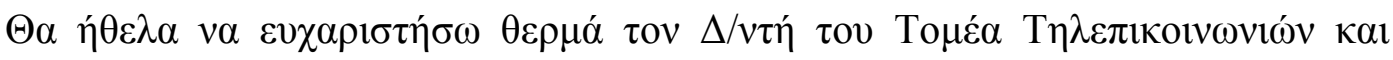

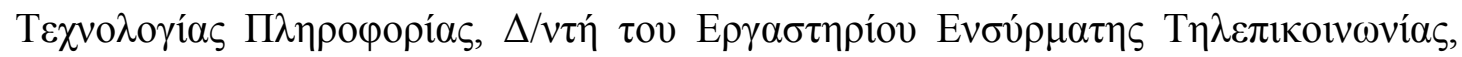

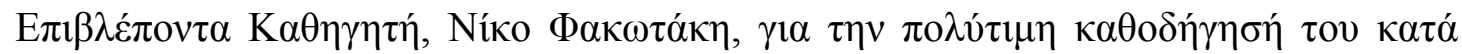

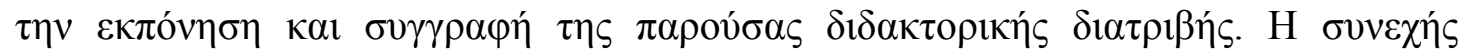

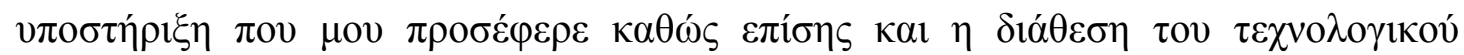

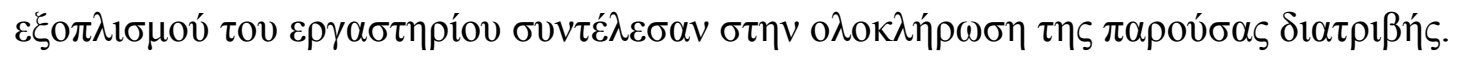

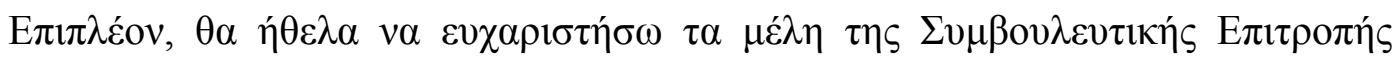

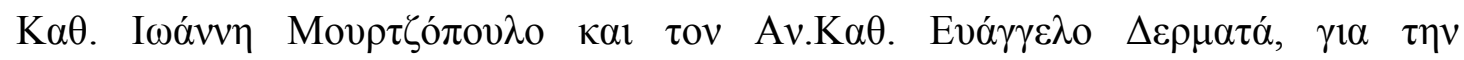

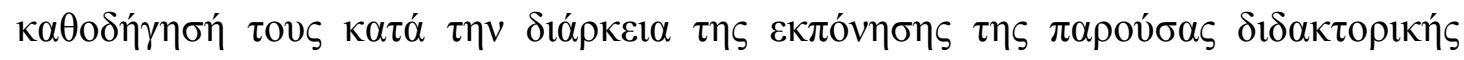

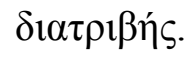

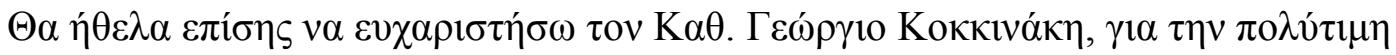

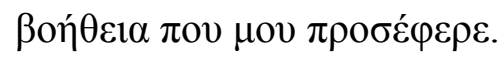

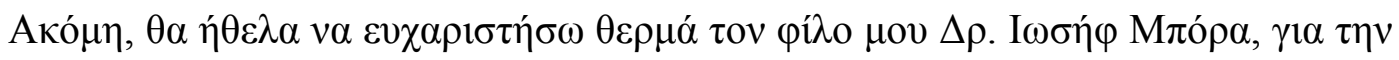

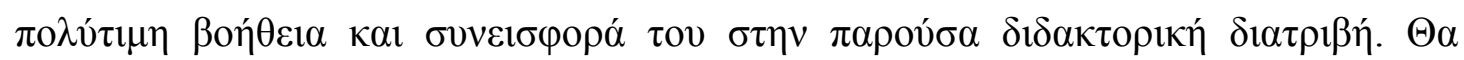

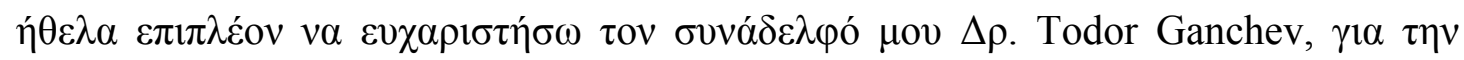

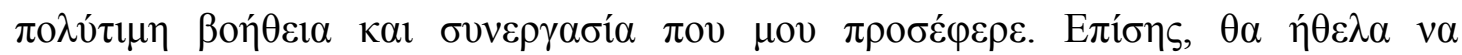

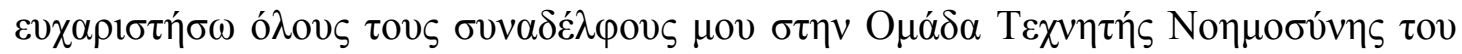

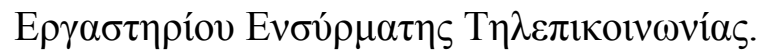

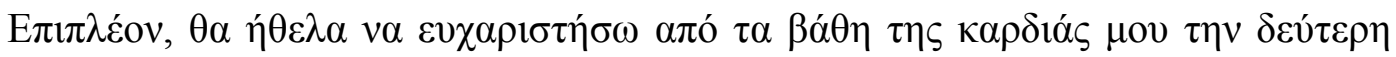

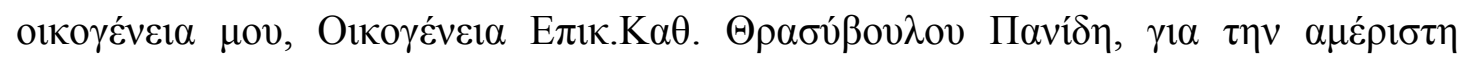

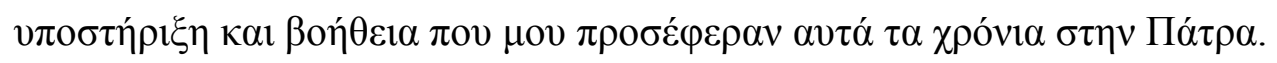

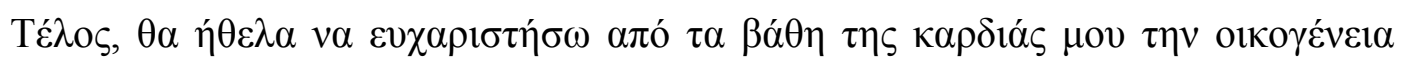

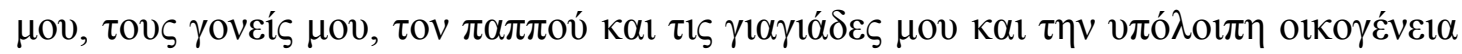

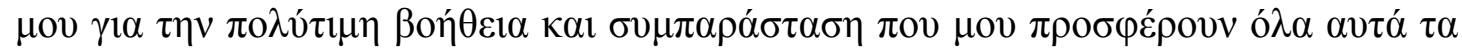

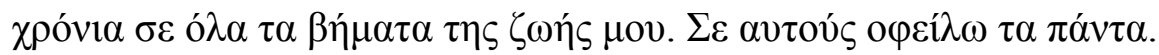




\section{Пєрí $\lambda \psi \psi \eta$}

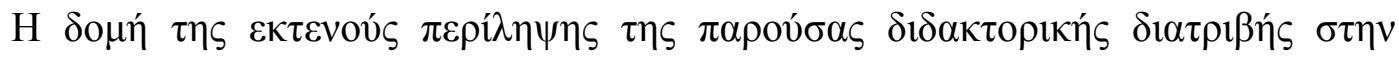

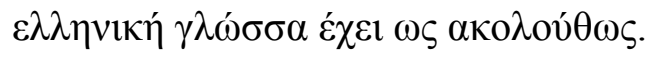

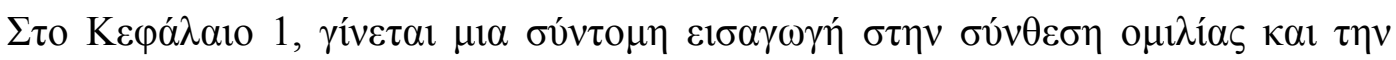

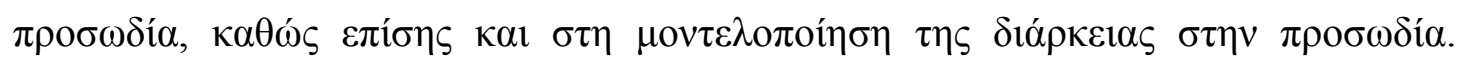

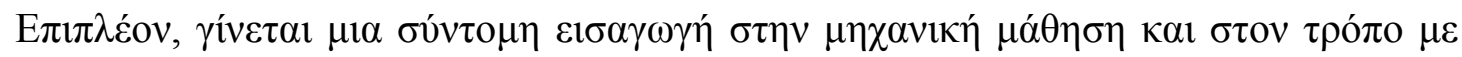

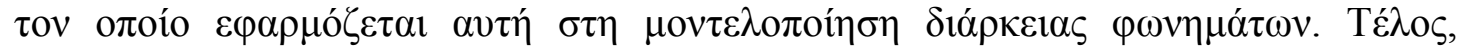

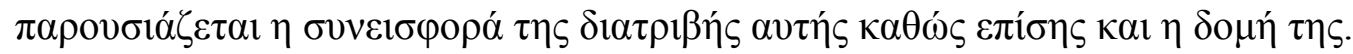

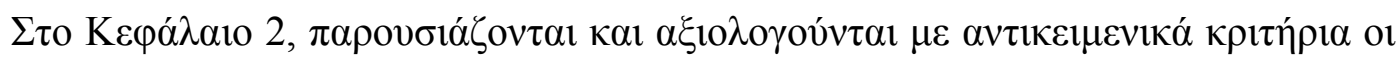

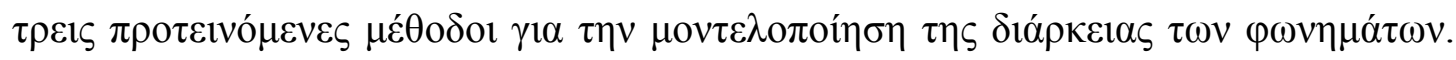

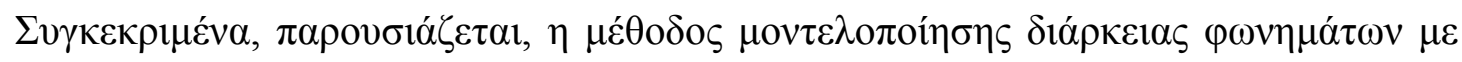

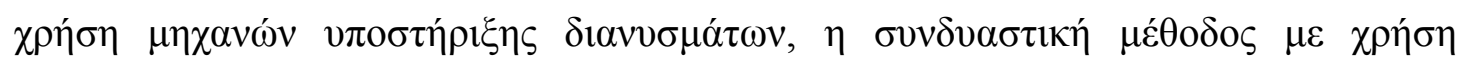

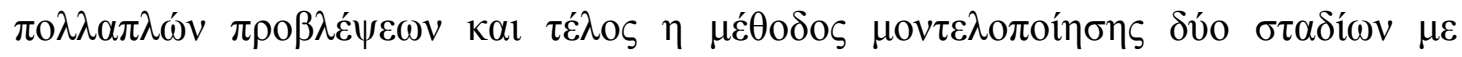

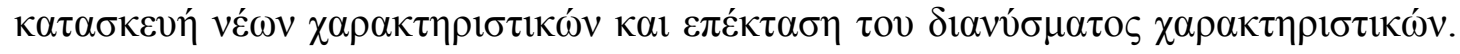

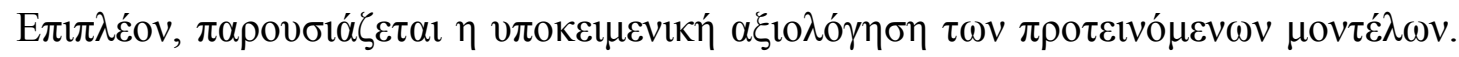

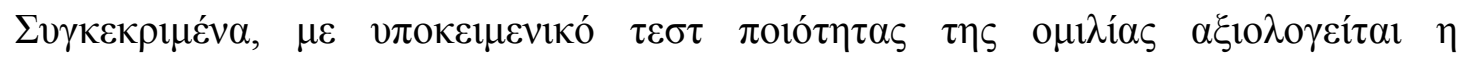

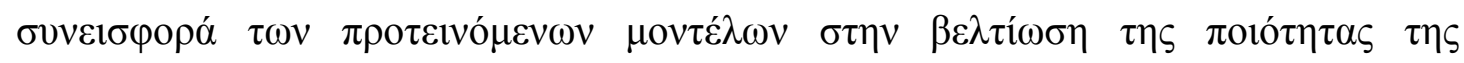

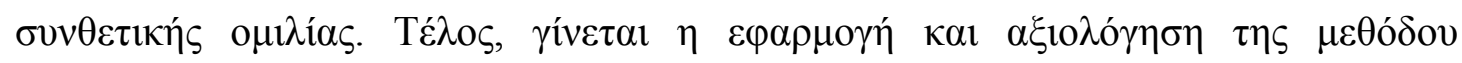

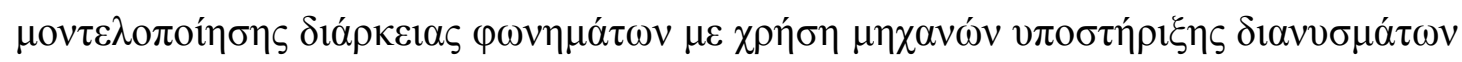

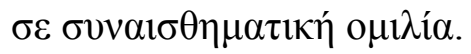

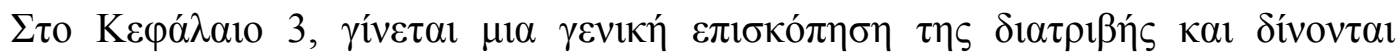

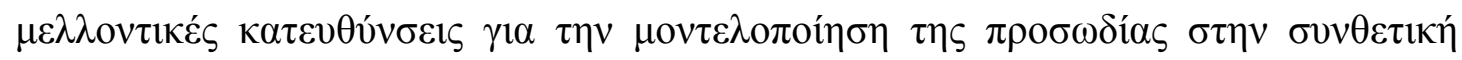

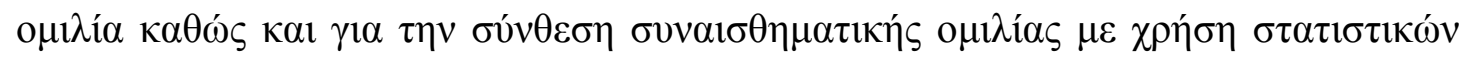

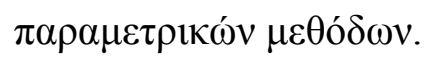

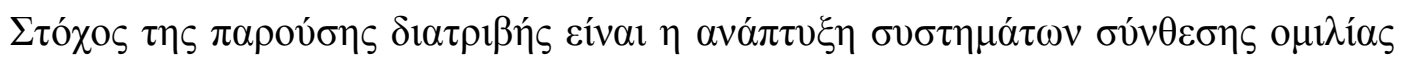

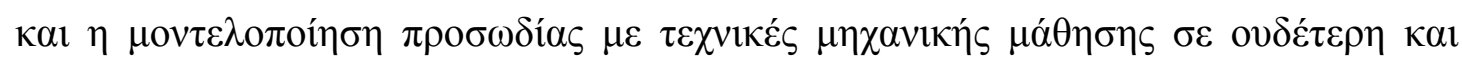

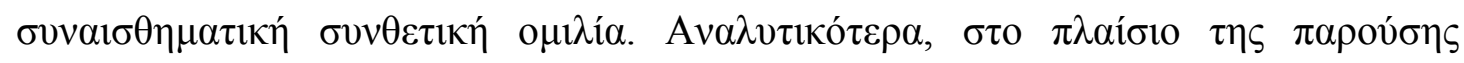

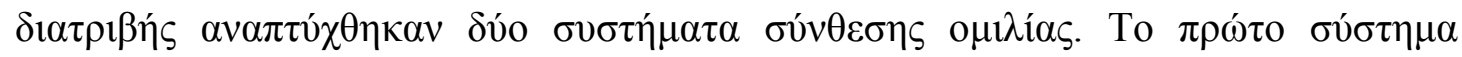

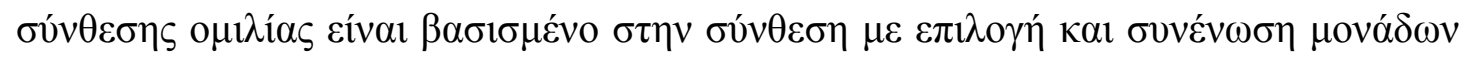

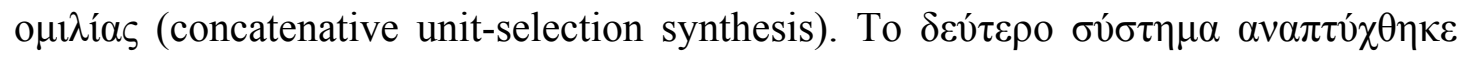

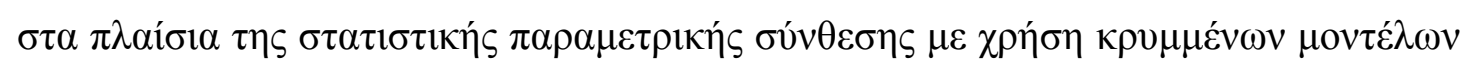

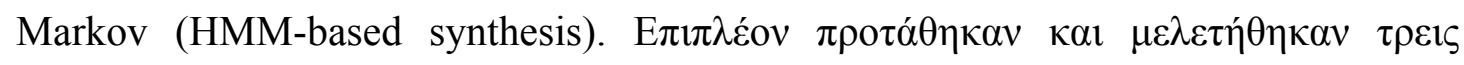




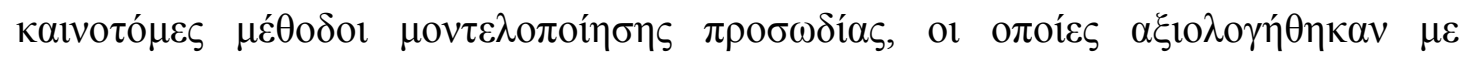

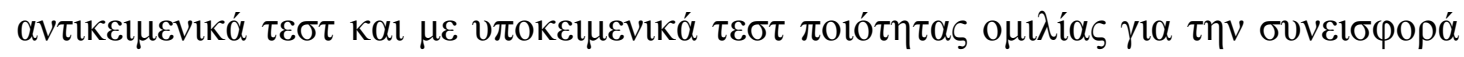

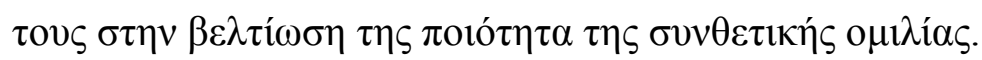

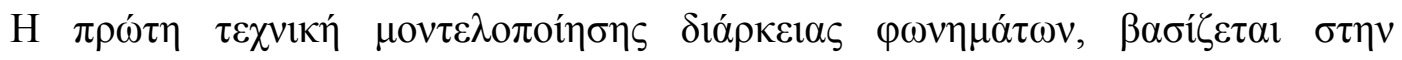

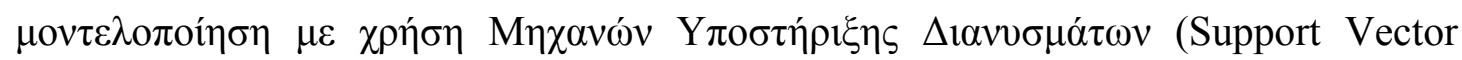

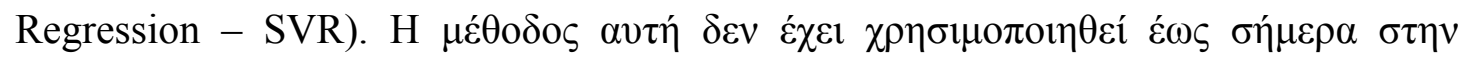

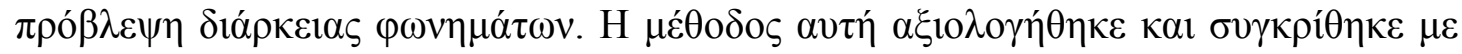

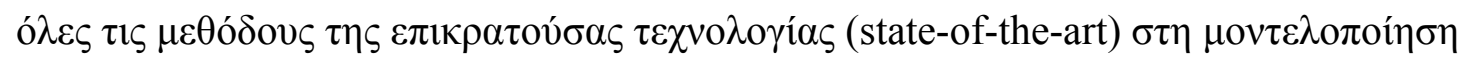

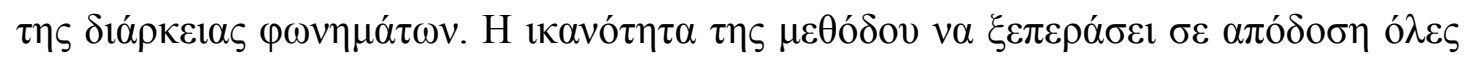

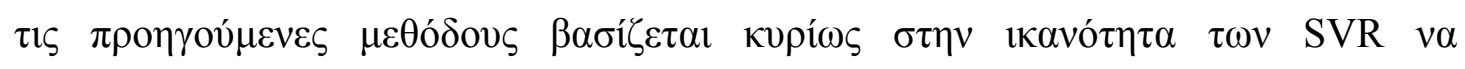

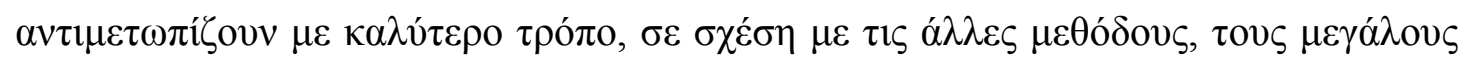

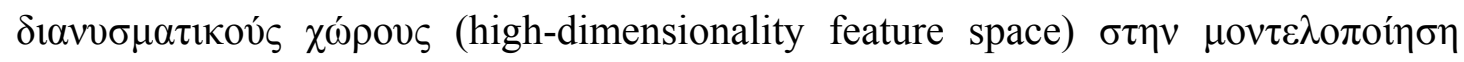

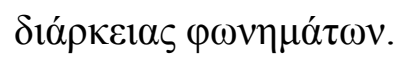

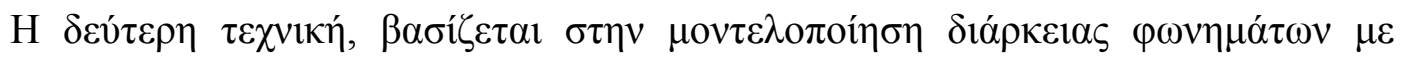

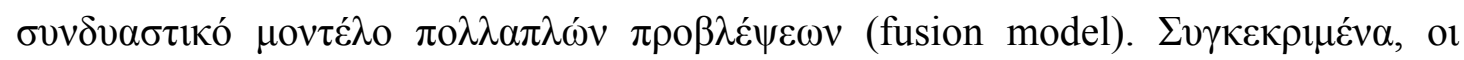

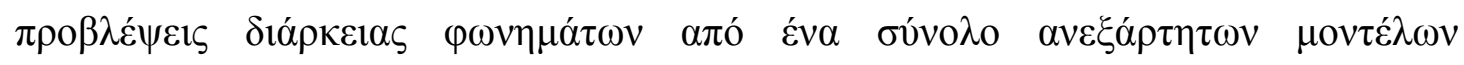

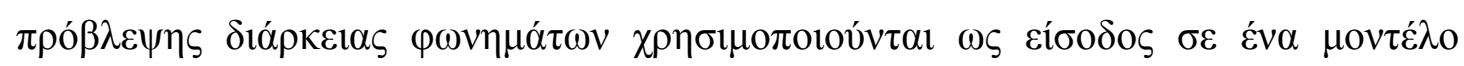

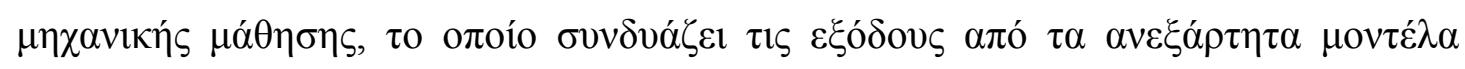

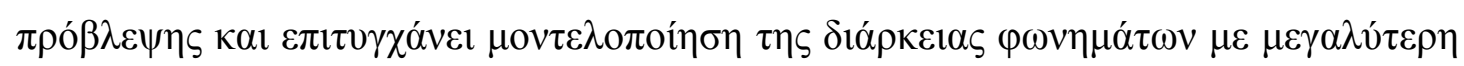

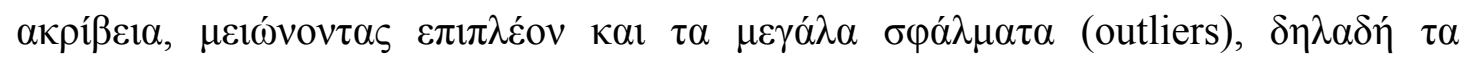

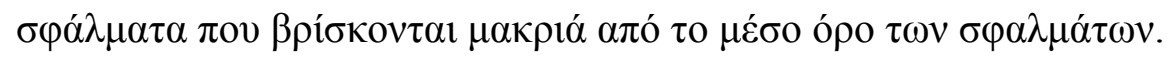

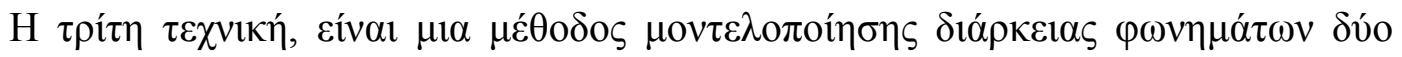

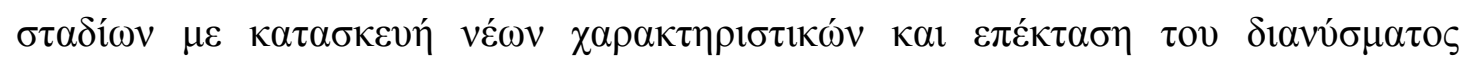

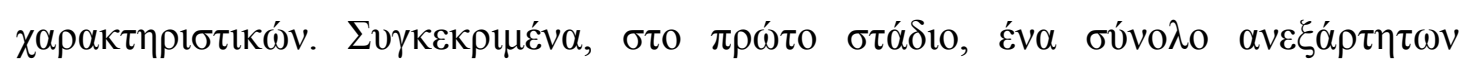

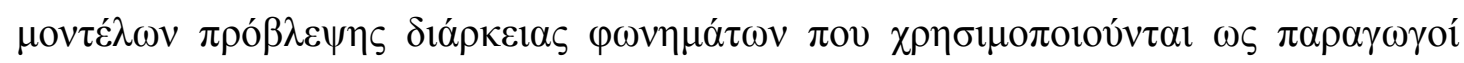

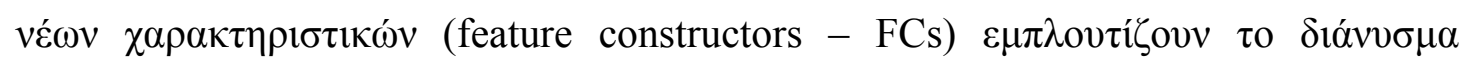

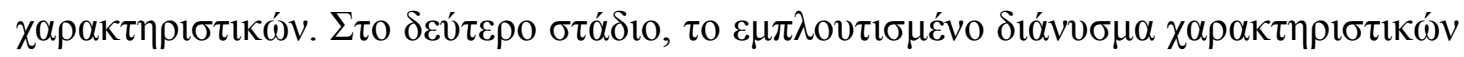

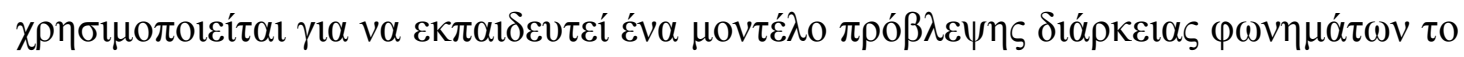

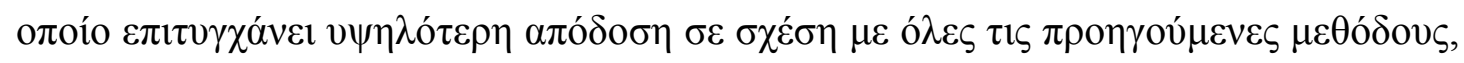

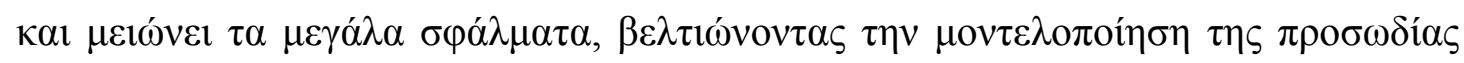

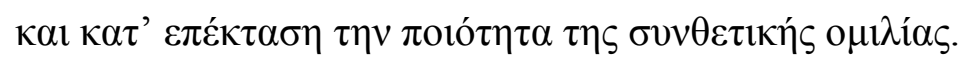

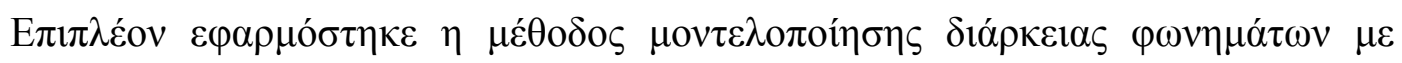

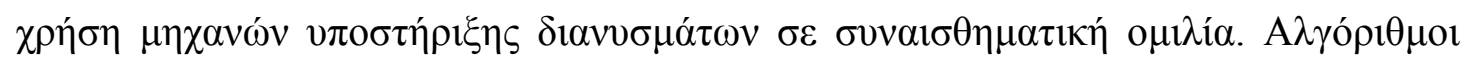

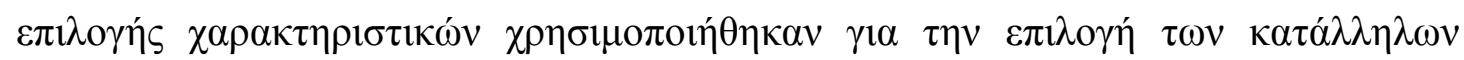




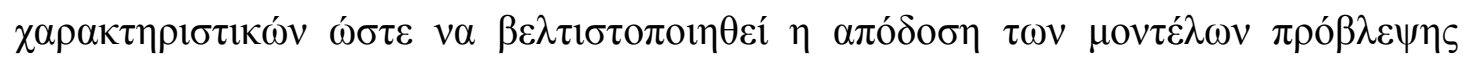

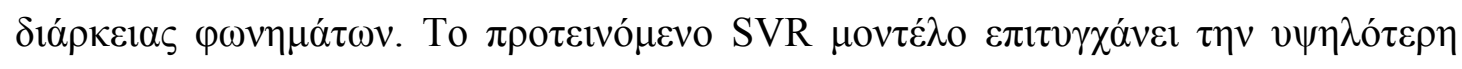

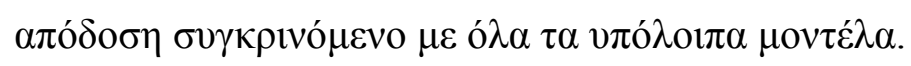

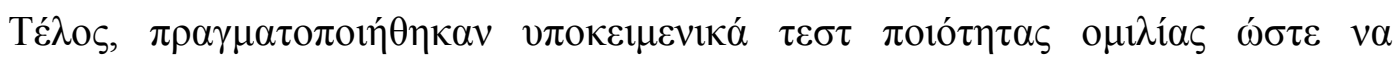

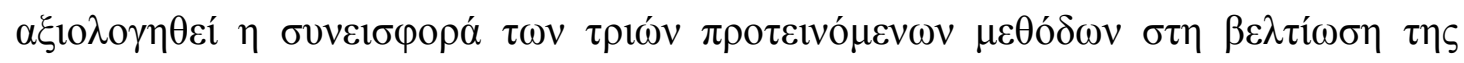

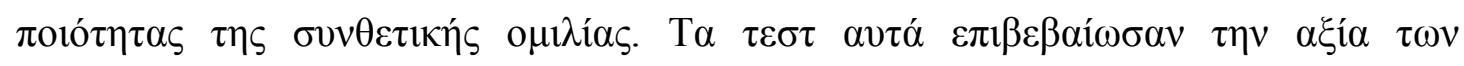

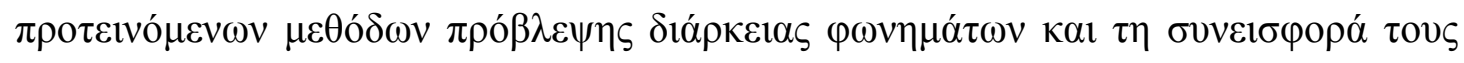

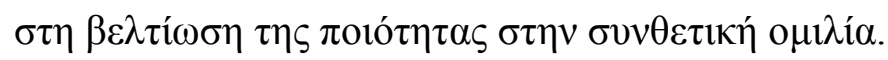




\section{ПEPIEXOMENA}

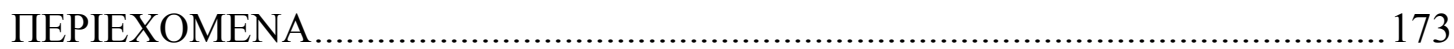

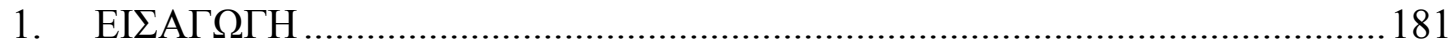

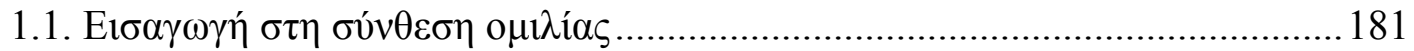

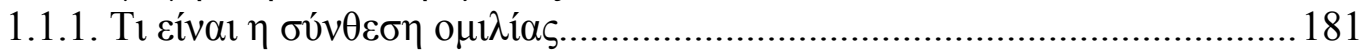

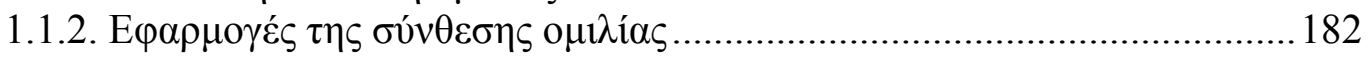

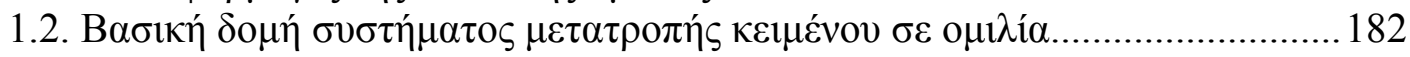

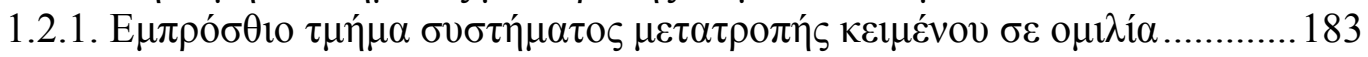

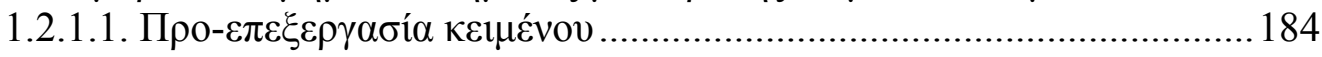

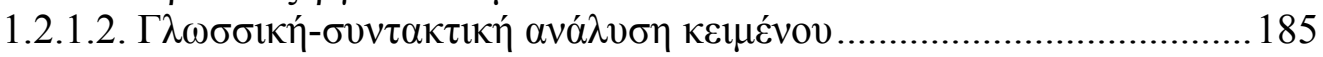

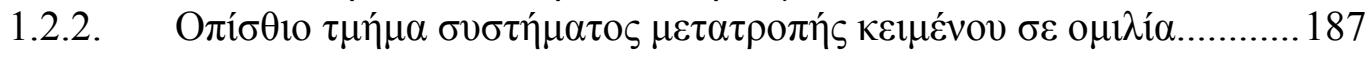

1.2.2.1. $\Sigma v ́ v \theta \varepsilon \sigma \eta \alpha ́ \alpha \rho \rho \omega \sigma \eta \varsigma$ (Articulatory Synthesis)....................................188

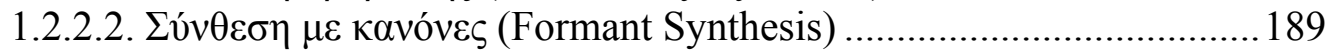

1.2.2.3. $\Sigma v ́ v \theta \varepsilon \sigma \eta \mu \varepsilon \sigma v v \varepsilon ́ v \omega \sigma \eta \mu$ ovó $\delta \omega v$ (Concatenative Synthesis) .............191

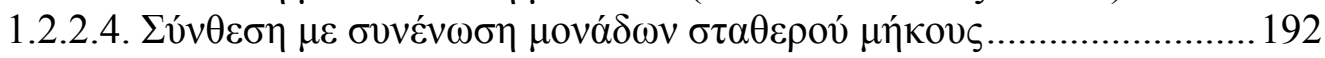

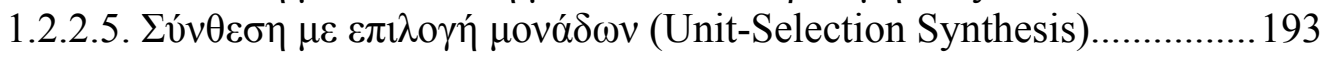

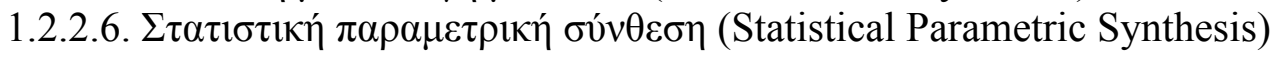

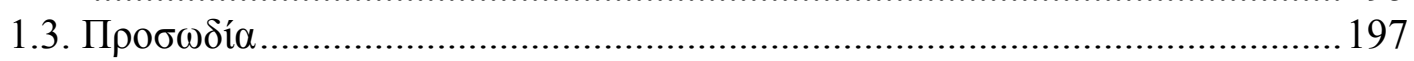

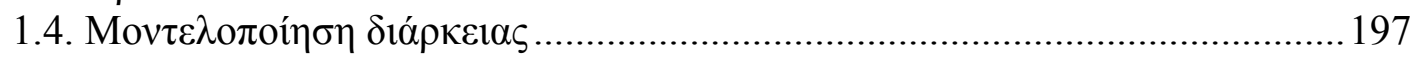

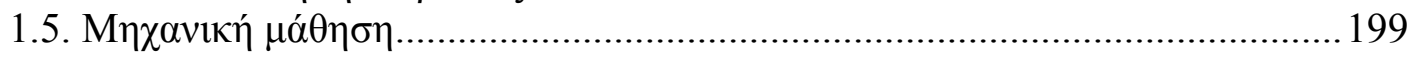

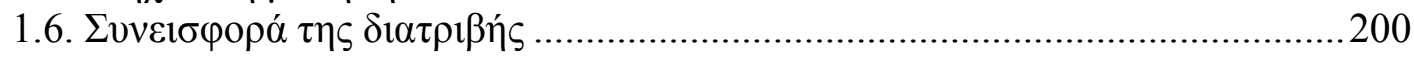

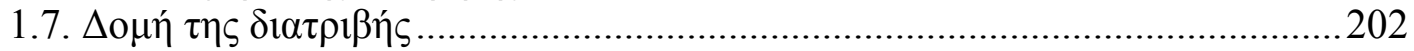

2. ПPOTEINOMENA MONTE $\Lambda$ A ПРО $\Sigma \Omega \Delta \mathrm{IA} \Sigma \mathrm{K}$ KAI MONTE $\Lambda$ OПOIH $\Sigma \mathrm{H}$

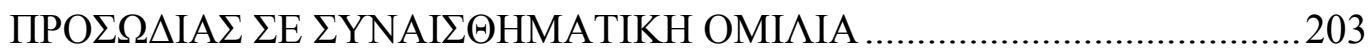

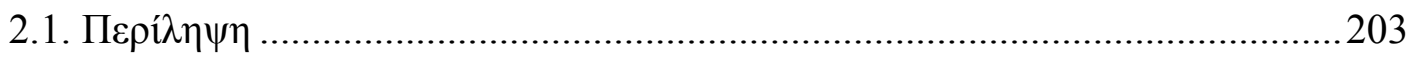

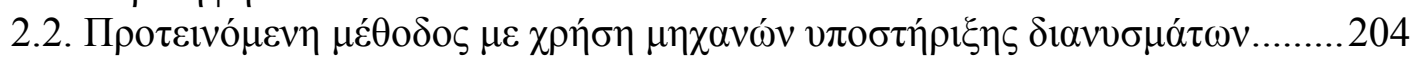

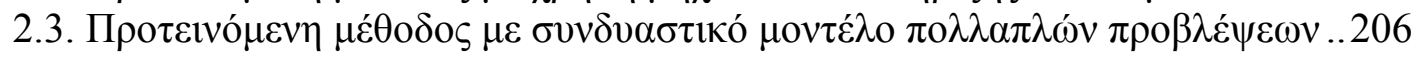

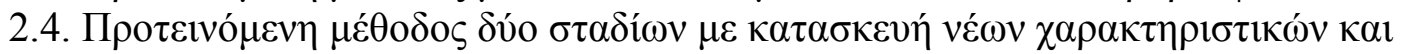

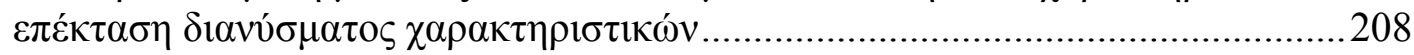

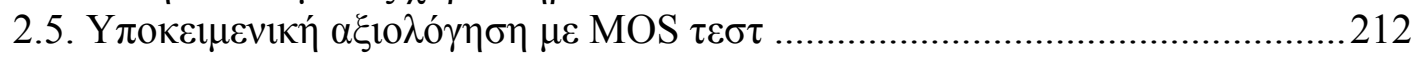

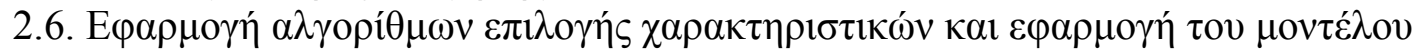

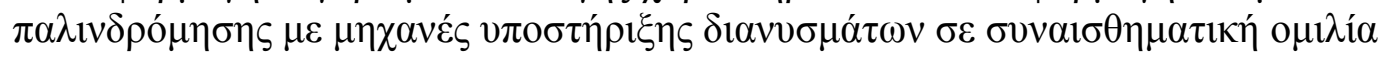

3. ANA $\Sigma$ KOПH $\Sigma H$ TH $\Sigma \Delta \mathrm{I} \Delta$ AKTOPIKH $\Sigma \Delta \mathrm{IATPIBH} \Sigma$ KAI ME $\Lambda \Lambda$ ONTIKE $\Sigma$

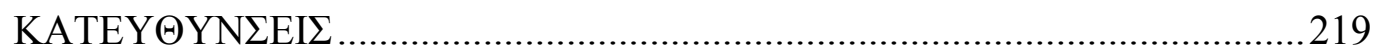

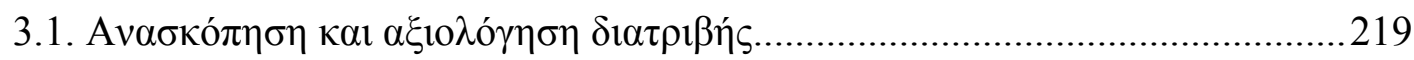

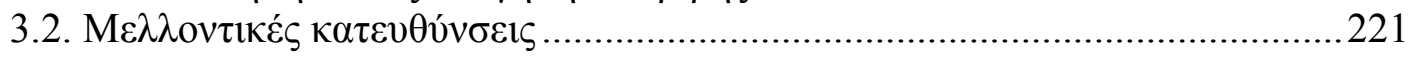

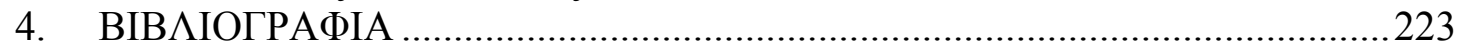




\section{$\Lambda i ́ \sigma \tau \alpha \Sigma \chi \eta \mu \alpha ́ \tau \omega v$}

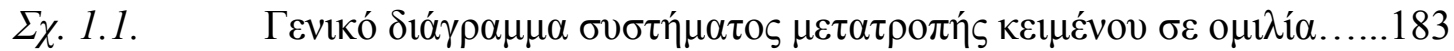

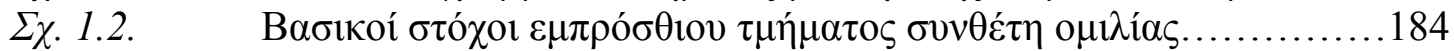

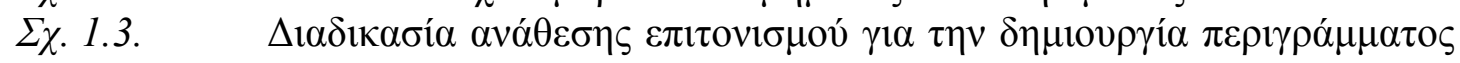

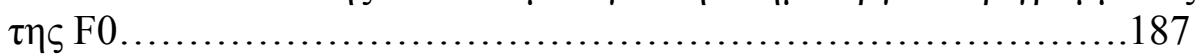

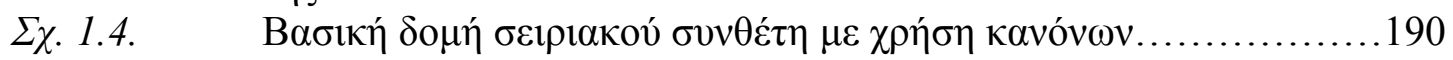

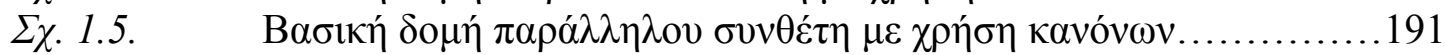

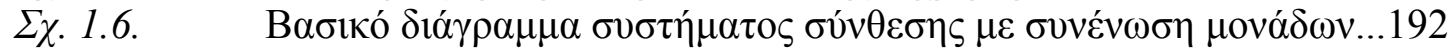

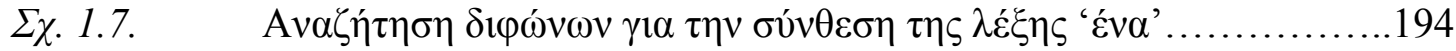

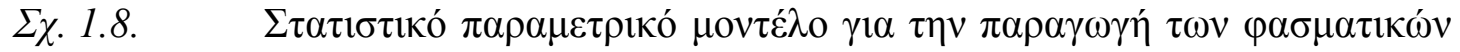

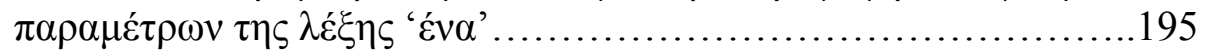

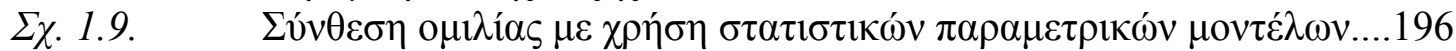

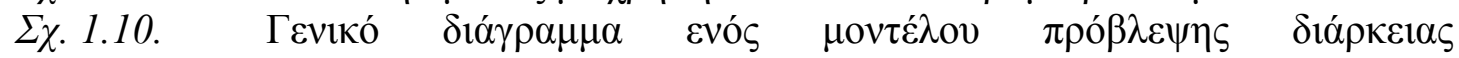

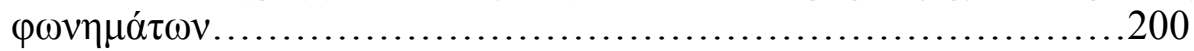

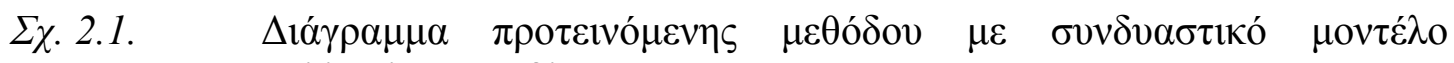

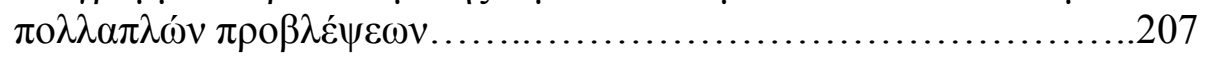

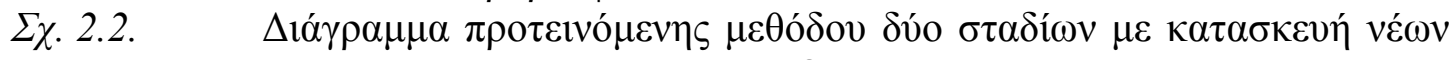

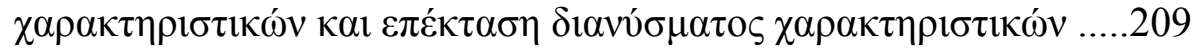

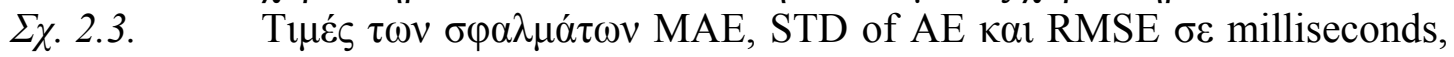

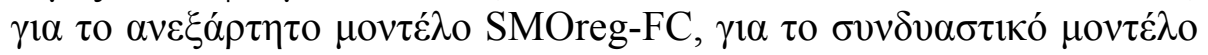

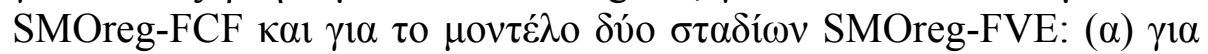

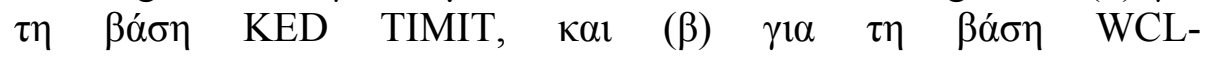

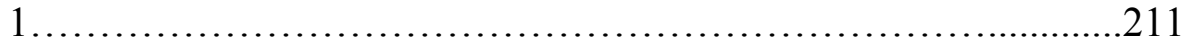




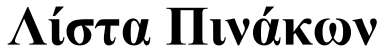

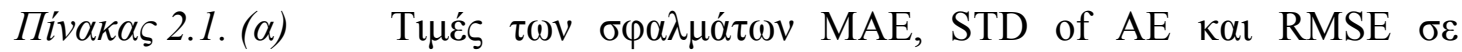

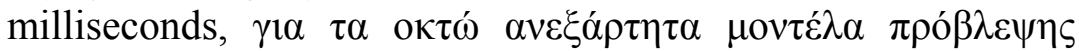

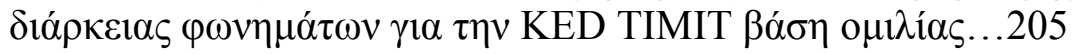

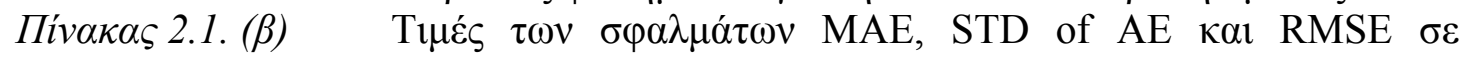

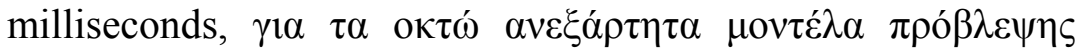

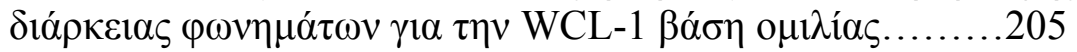

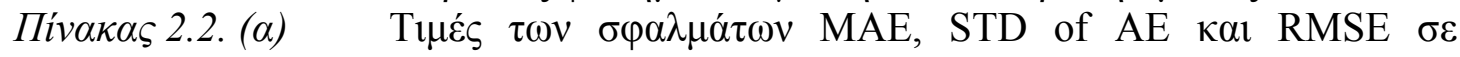

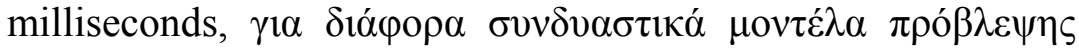

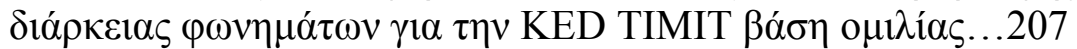

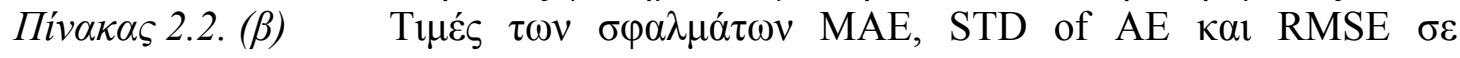

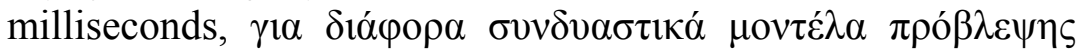

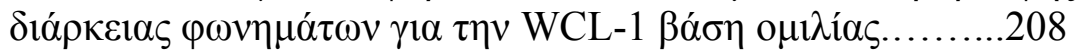

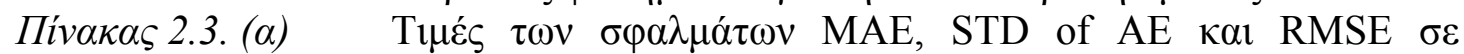

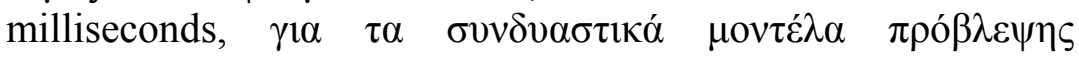

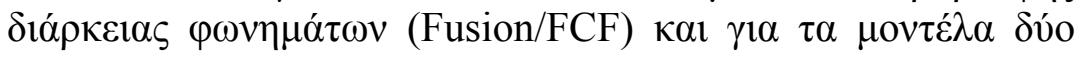
$\sigma \tau \alpha \delta i ́ \omega v(\mathrm{FVE}) \gamma i \alpha \tau \eta \nu$ KED TIMIT $\beta \alpha ́ \sigma \eta ~ o \mu \imath \lambda i ́ \alpha \varsigma . . . . . . . . .210$

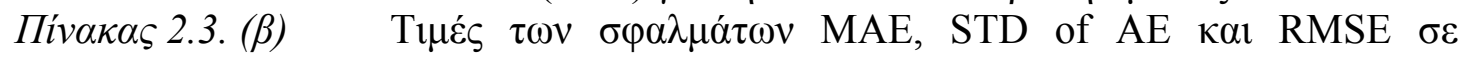

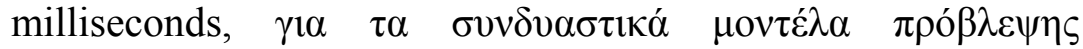

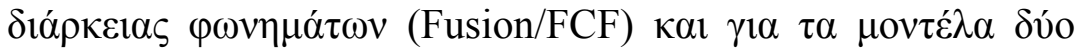

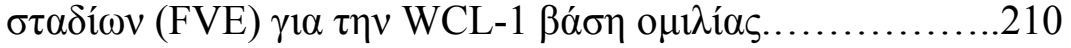

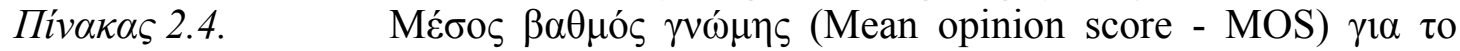

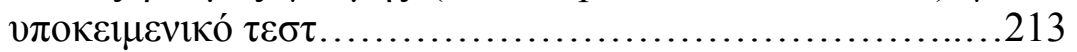

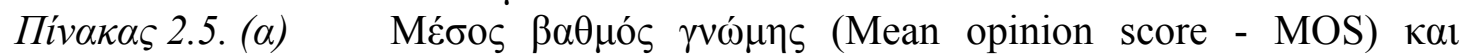

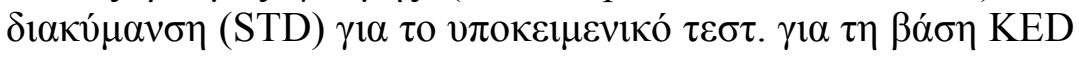
TIMIT...................................................213

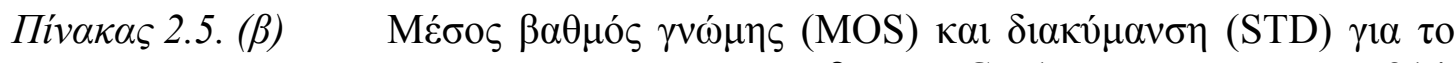

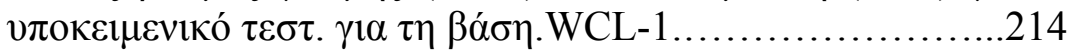

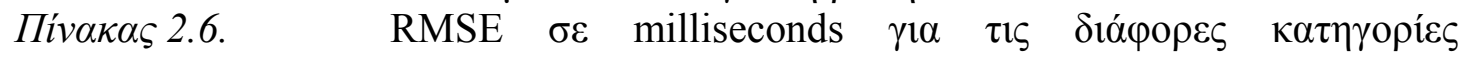

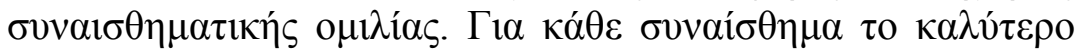

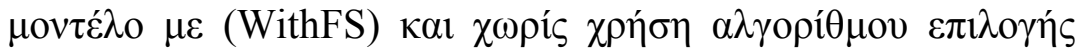

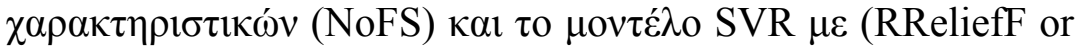

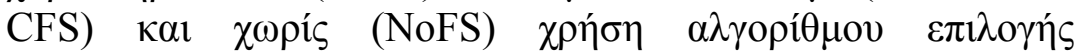

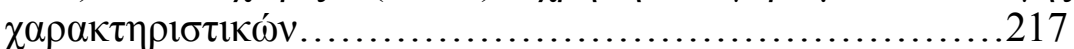

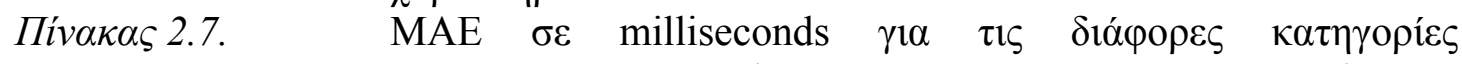

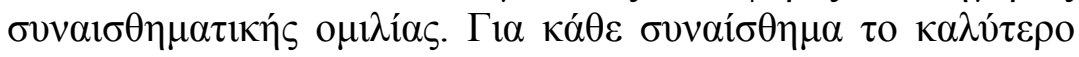

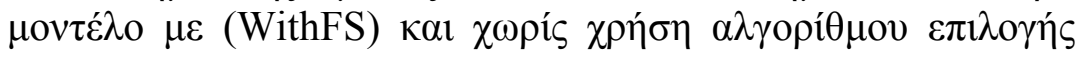

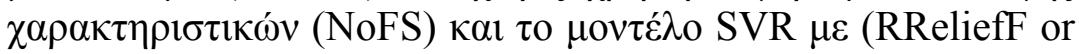

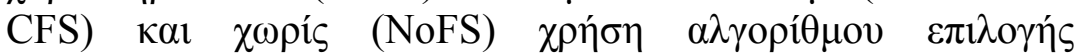

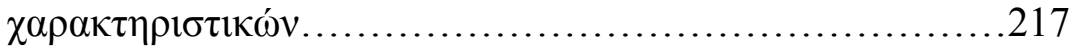

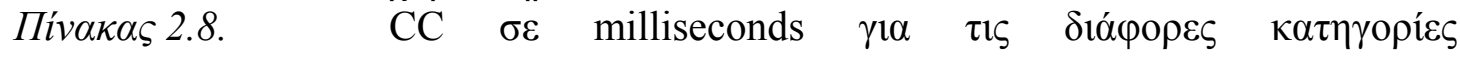

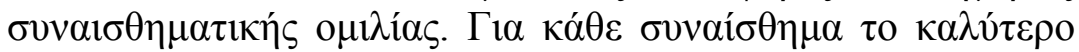

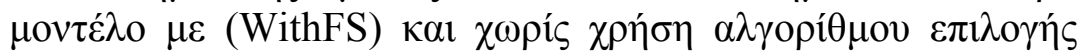

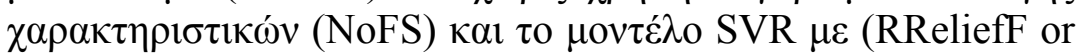

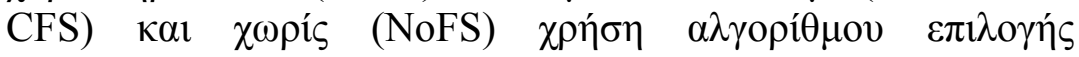

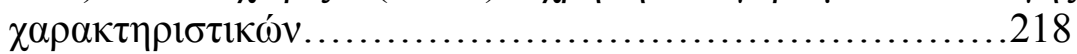




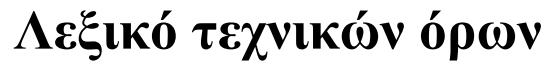

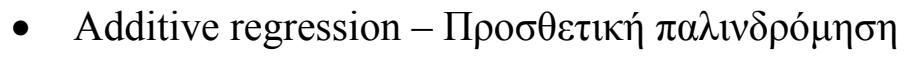

- Articulatory synthesis - $\Sigma v ́ v \theta \varepsilon \sigma \eta \eta \dot{\alpha} \rho \theta \rho \omega \sigma \eta \varsigma$

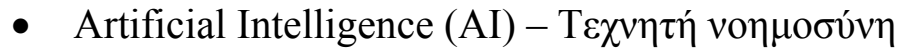

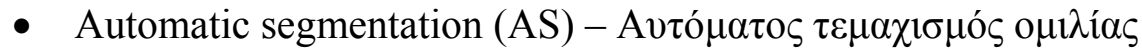

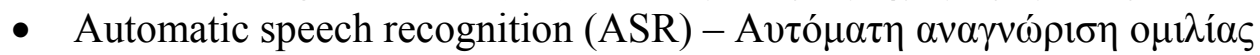

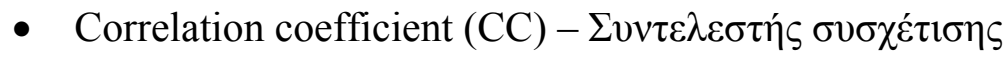

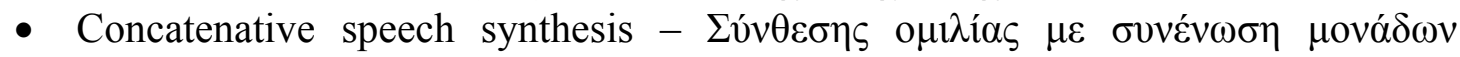

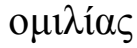

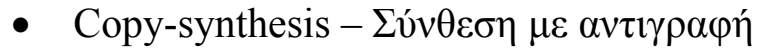

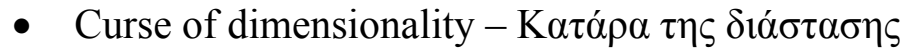

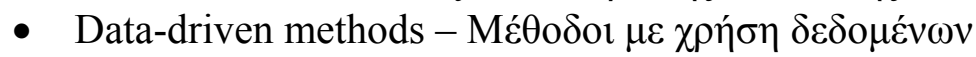

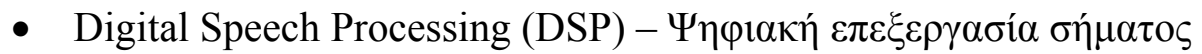

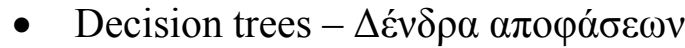

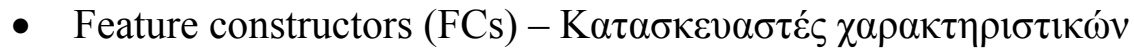

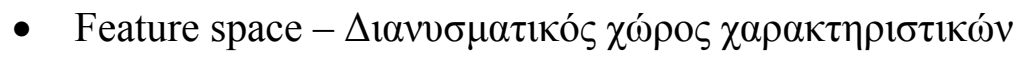

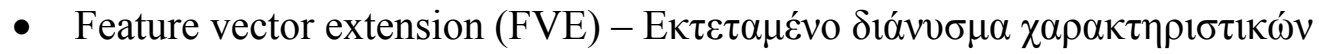

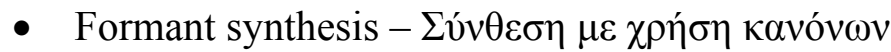

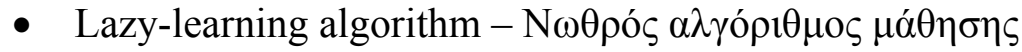

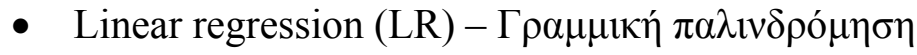

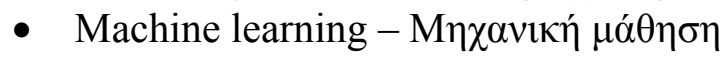

- Mean absolute error (MAE) - MÉбo $\alpha \pi$

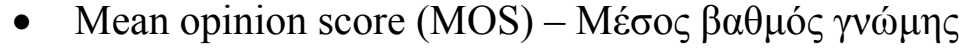

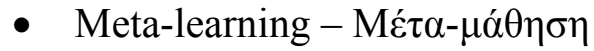

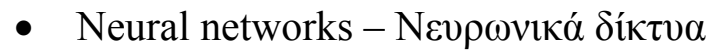

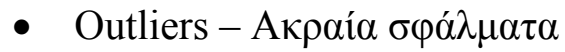

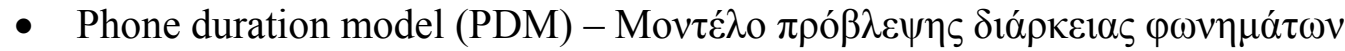

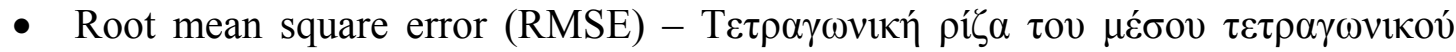
$\sigma \varphi \alpha ́ \lambda \mu \alpha \tau o \zeta$

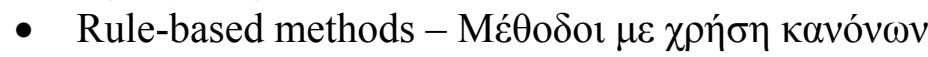

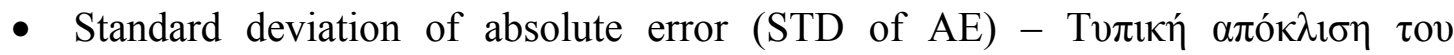
$\alpha \pi \hat{\lambda} \nu \tau \sigma o v \sigma \varphi \alpha ́ \lambda \mu \alpha \tau o \varsigma$

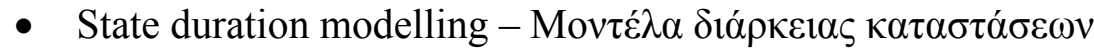

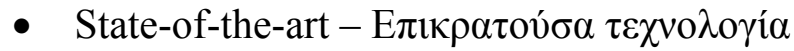

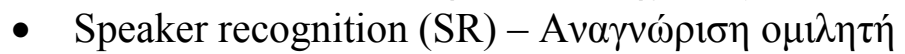

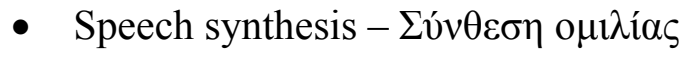

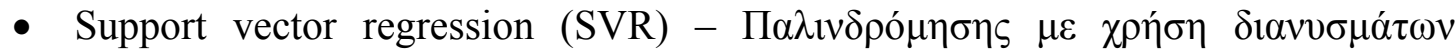

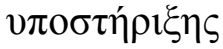

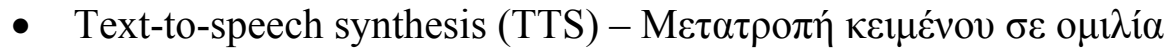

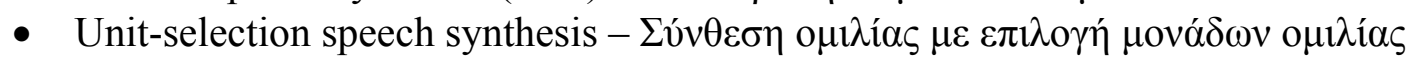




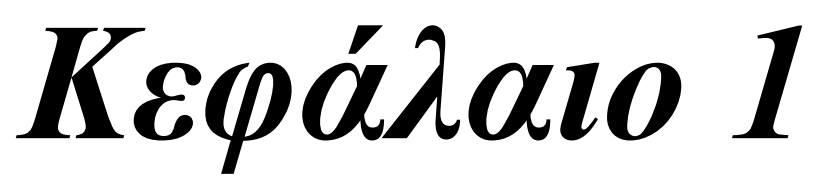

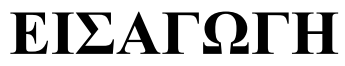

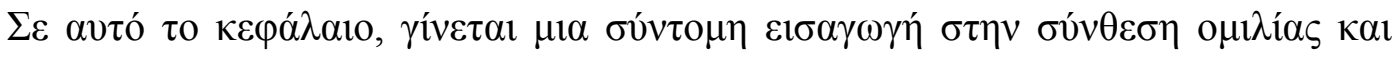

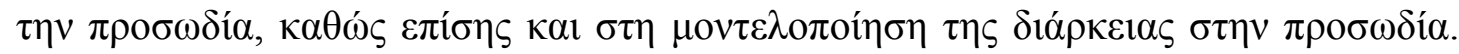

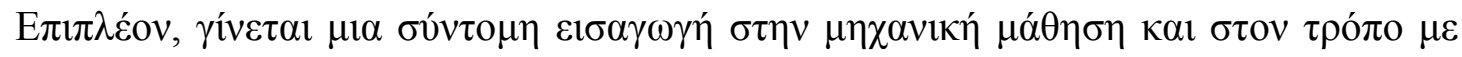

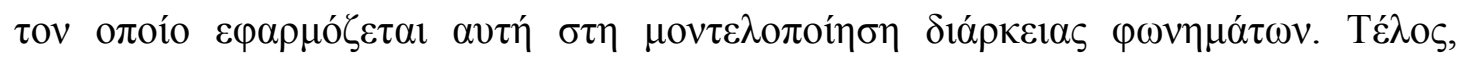

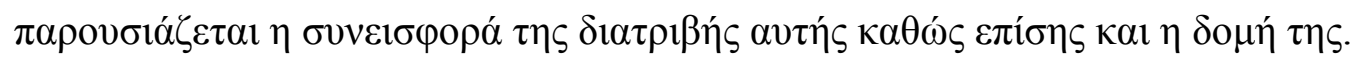

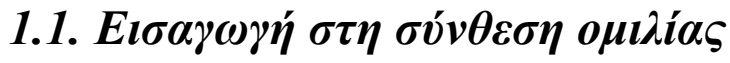

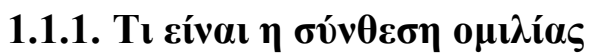

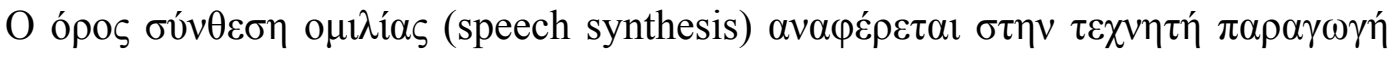

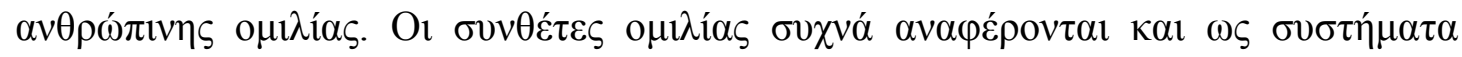

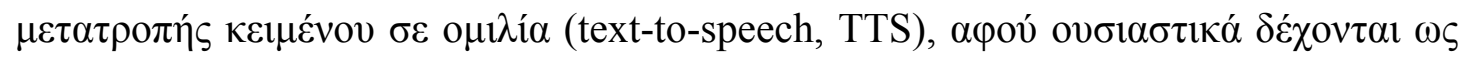

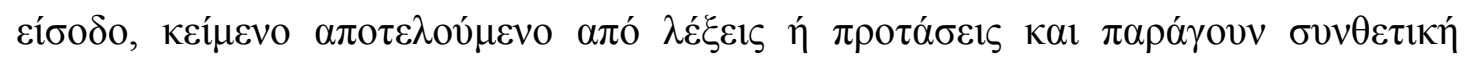

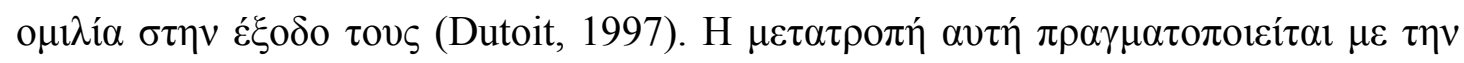

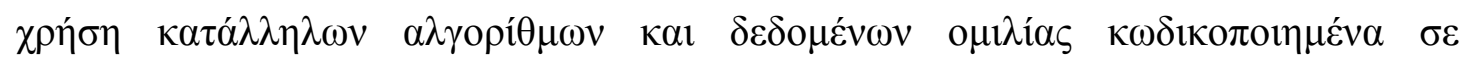

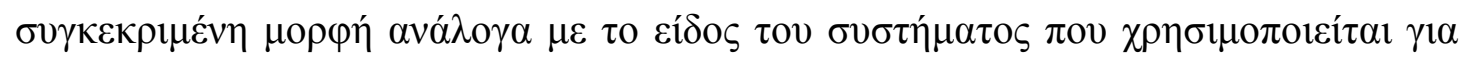

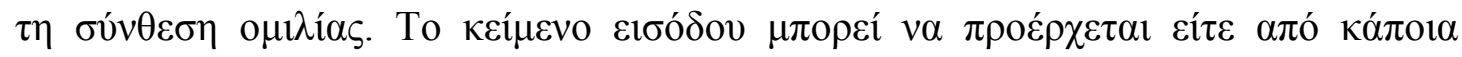

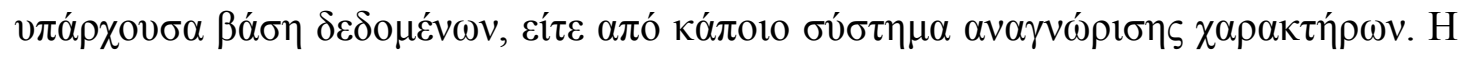




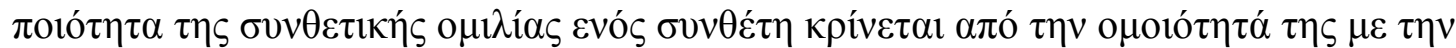

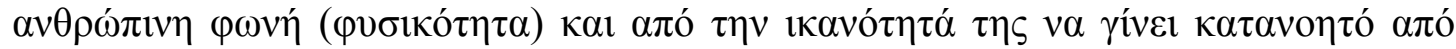

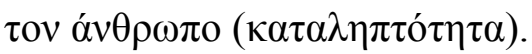

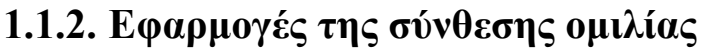

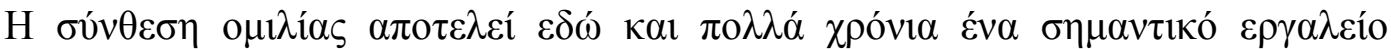

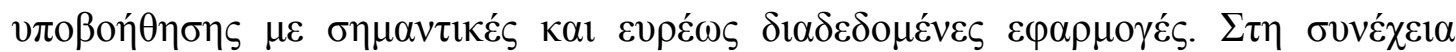

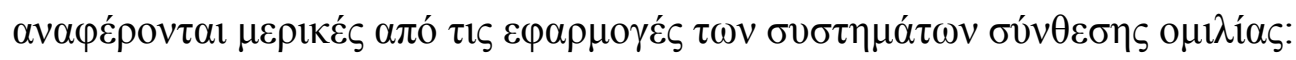

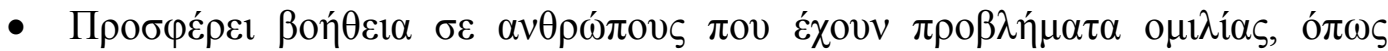

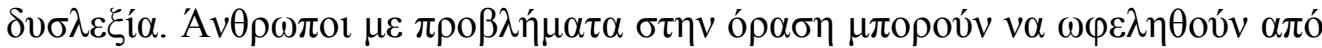

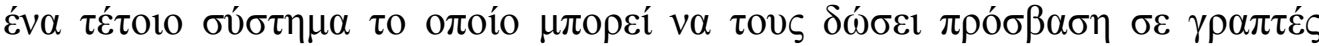

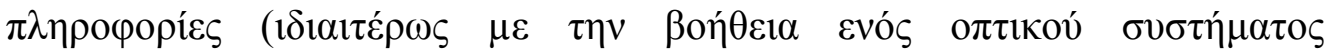

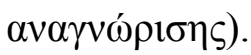

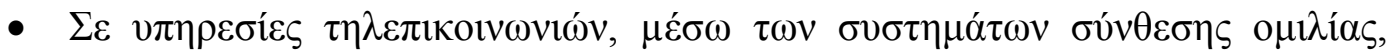

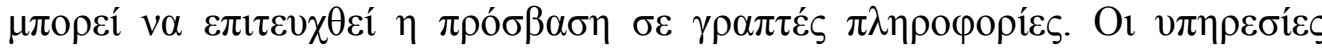

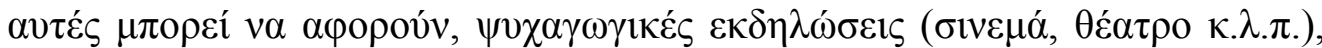

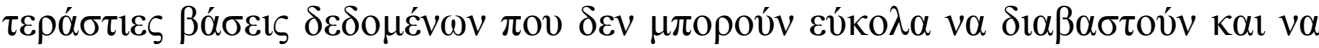

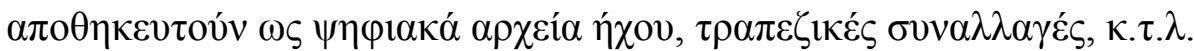

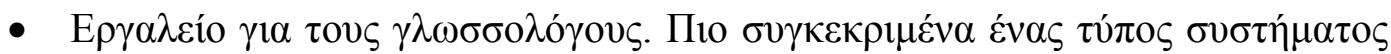

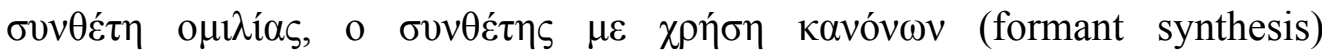

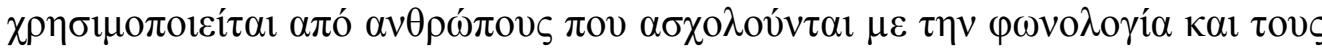

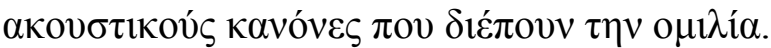

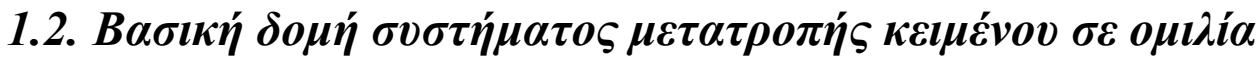

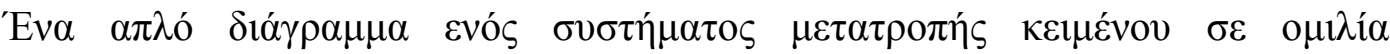

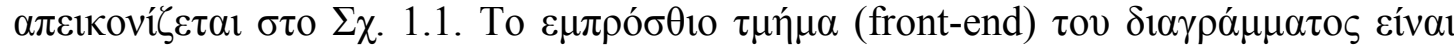

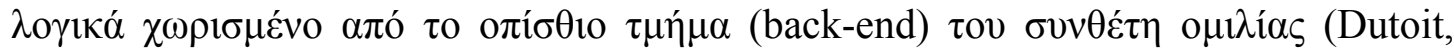

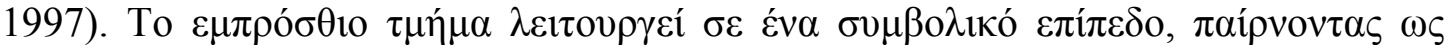
$\varepsilon 1 \sigma \alpha \gamma \omega \gamma \eta \dot{~ \kappa \varepsilon i ́ \mu \varepsilon v o ~ \kappa \alpha l ~ \pi \alpha \rho \alpha ́ \gamma о \nu \tau \alpha \varsigma ~ \pi \lambda \eta \rho о \varphi о \rho i ́ \varepsilon \varsigma ~ \varepsilon \lambda \varepsilon ́ \gamma \chi о v ~ \sigma \tau \eta v ~ \varepsilon ́ \xi o \delta o ́ ~ \tau o v . ~ T o ~ o \pi i ́ \sigma \theta 1 o ~}$ 


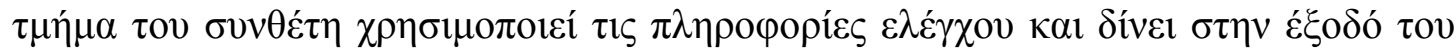

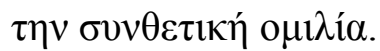

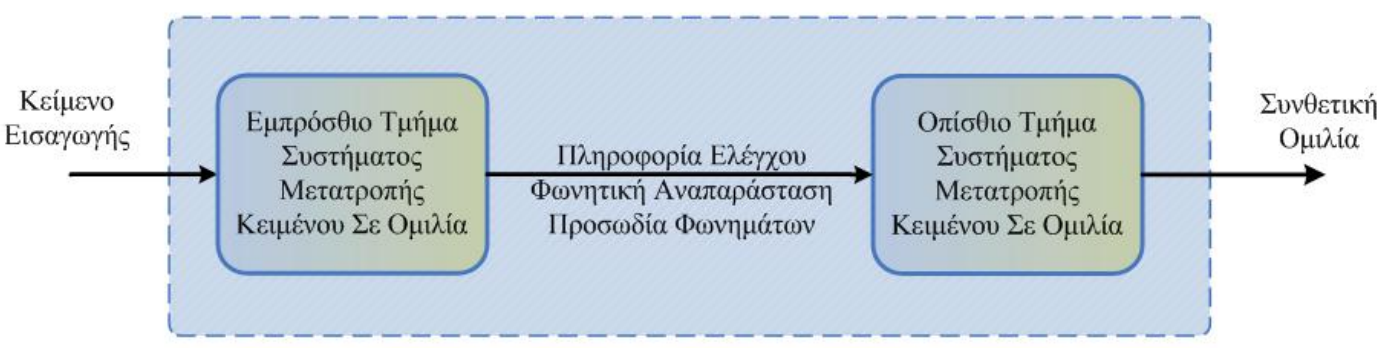

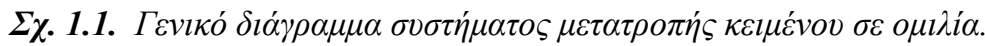

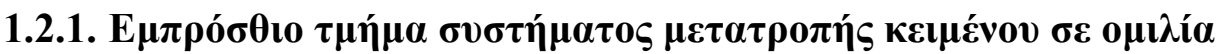

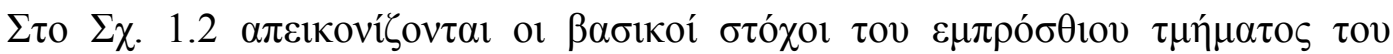

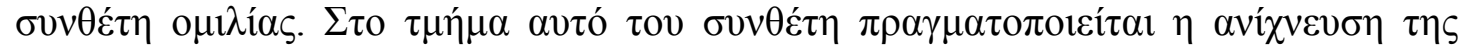

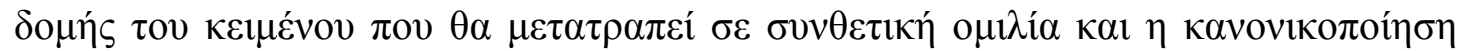

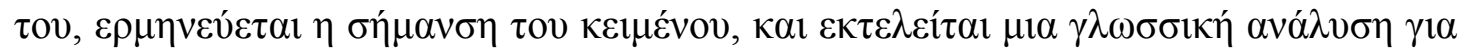

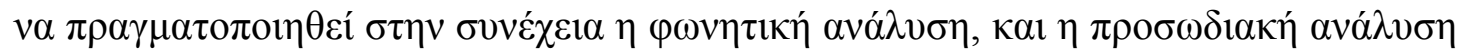

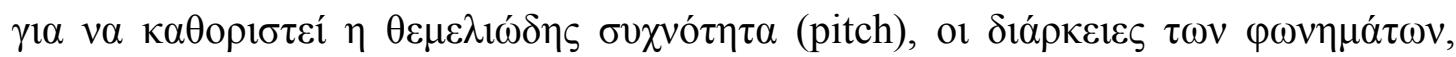

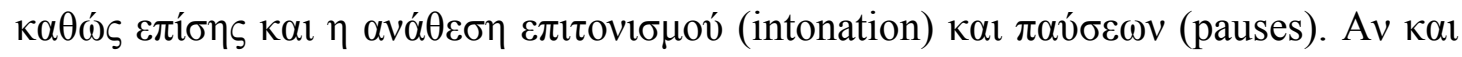

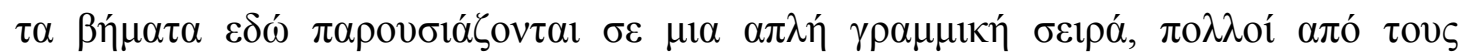

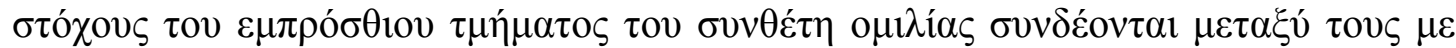

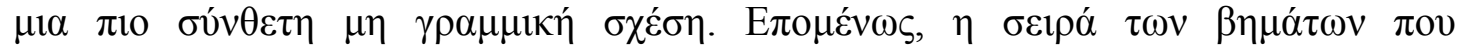

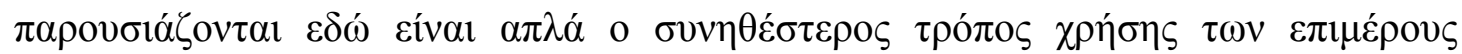

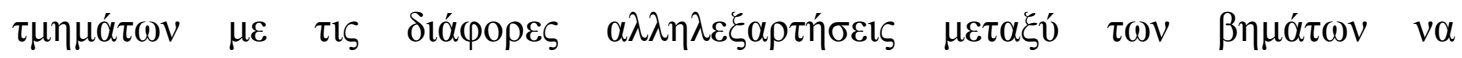

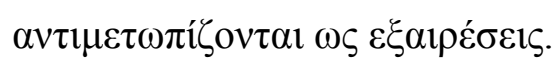




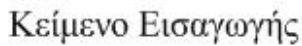

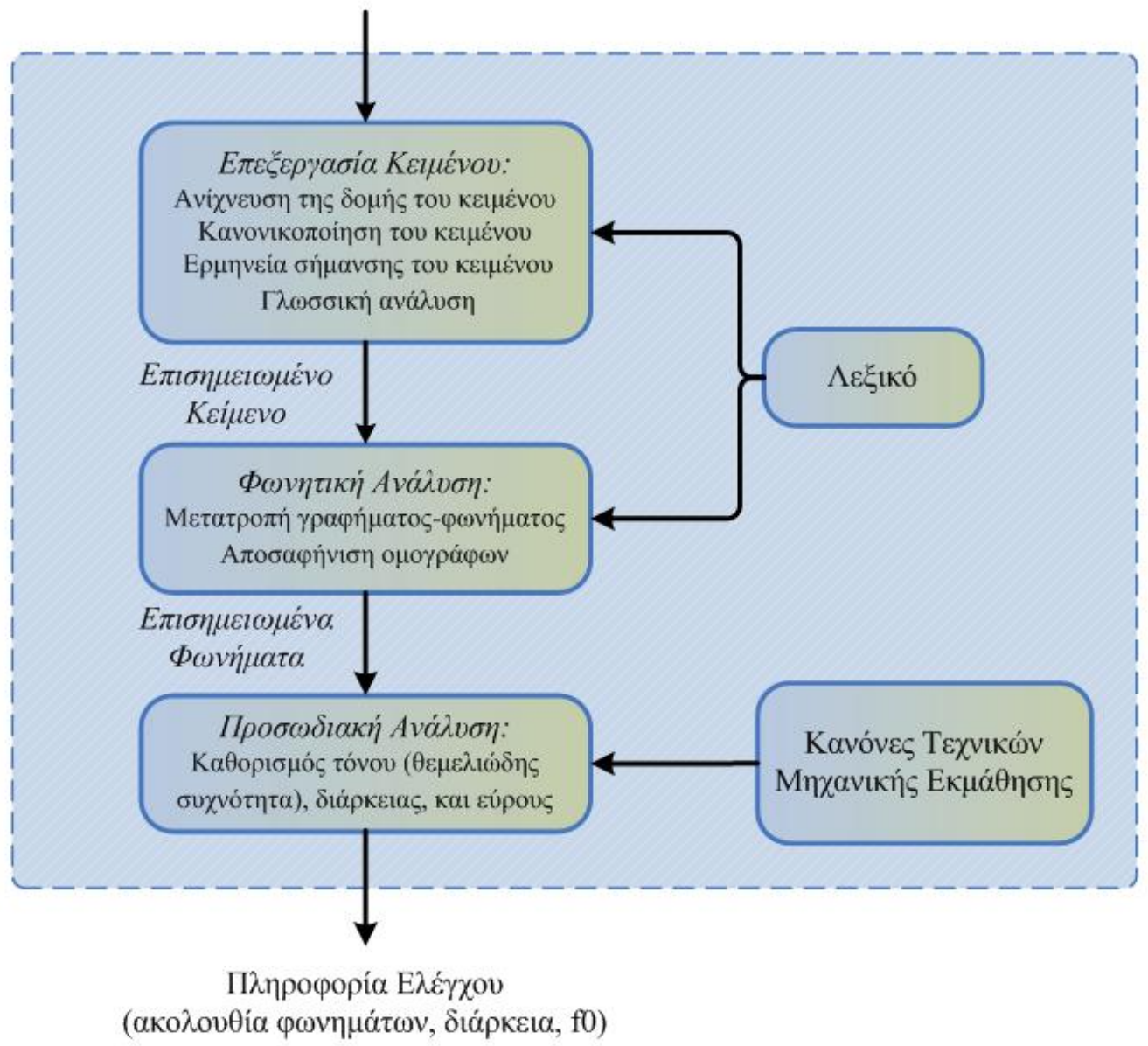

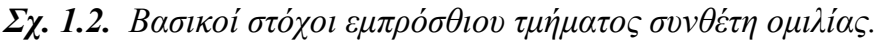

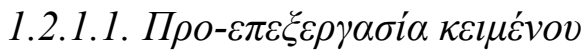

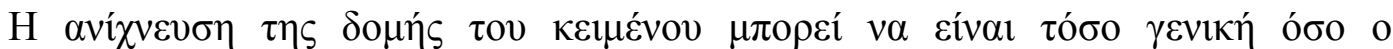

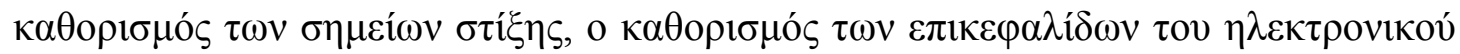

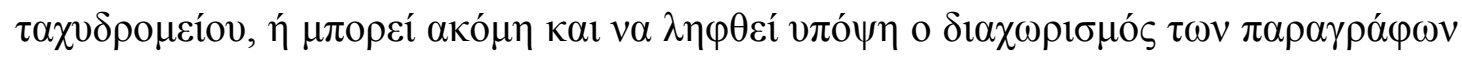

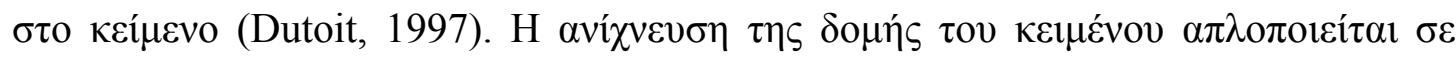

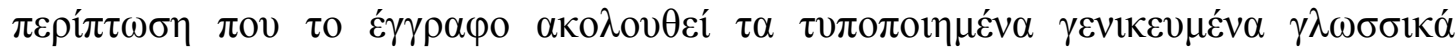

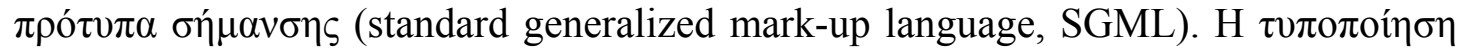

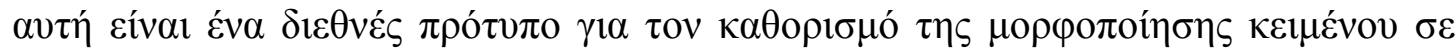

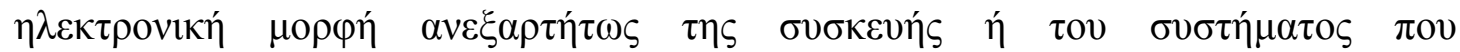

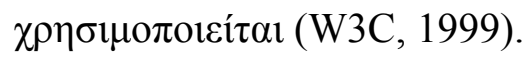




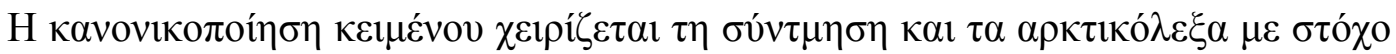

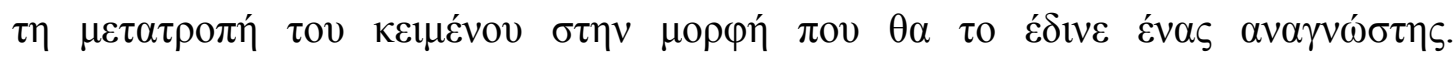

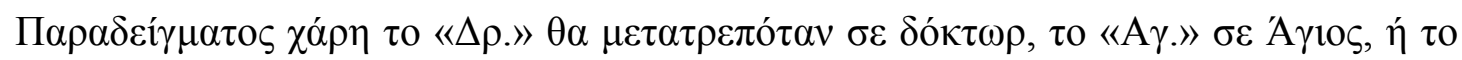
$\Delta \mathrm{EH} \sigma \varepsilon \delta \eta \mu o ́ \sigma ı \alpha \varepsilon \pi \imath \varepsilon \varepsilon i ́ \rho \eta\rceil \eta \lambda \varepsilon \kappa \tau \rho \imath \sigma \mu o u ́$.

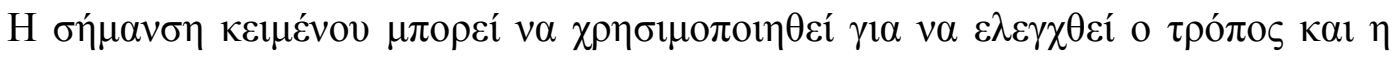

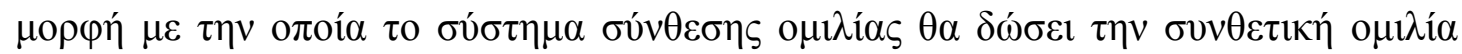

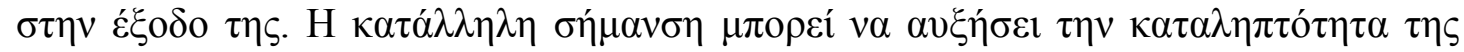

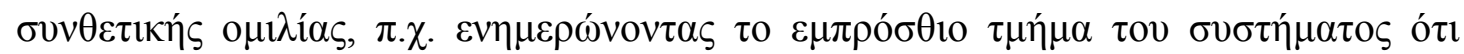

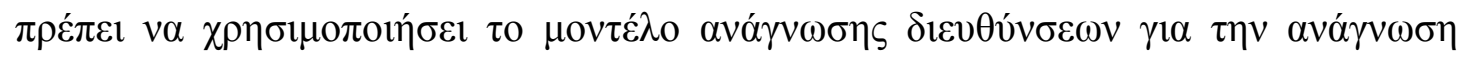

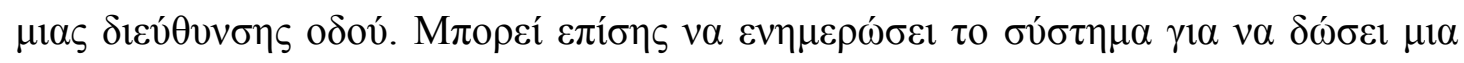

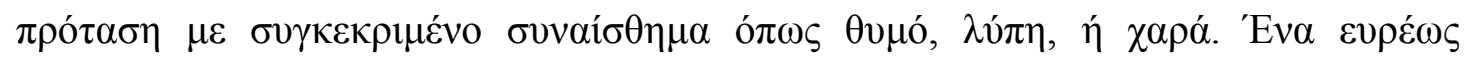

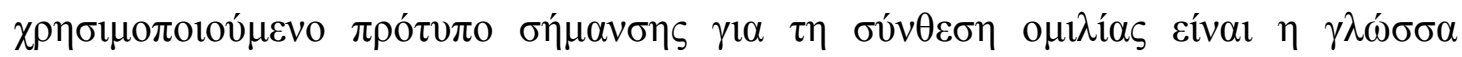

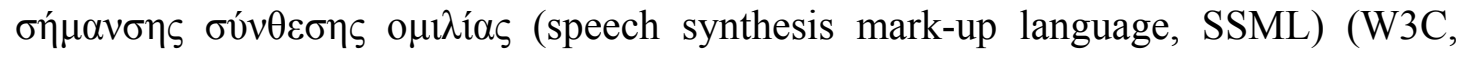
2003).

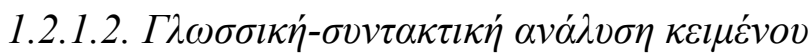

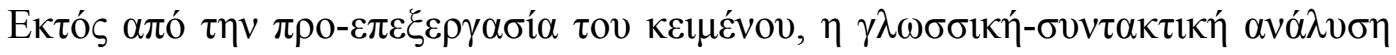

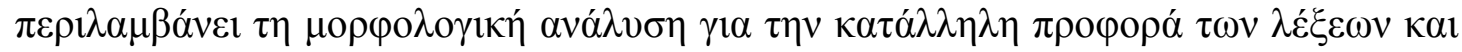

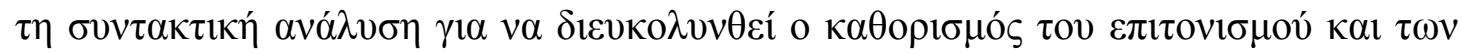

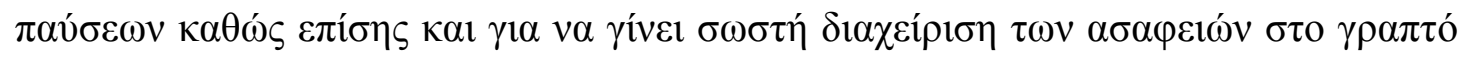

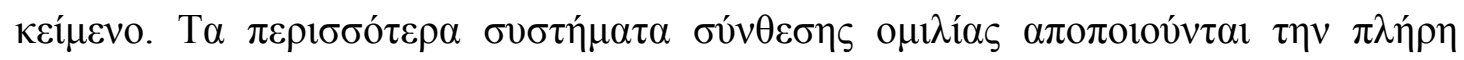

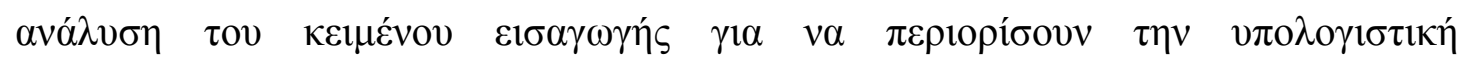

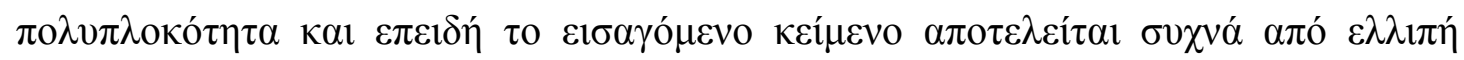

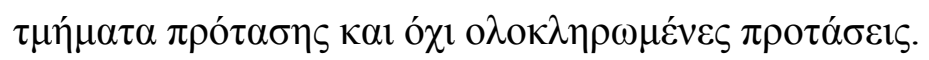

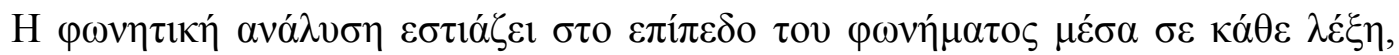

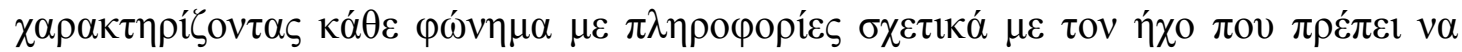

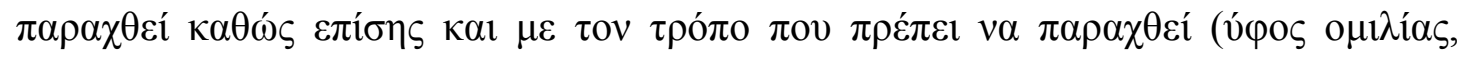

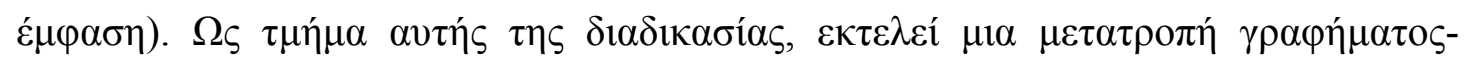

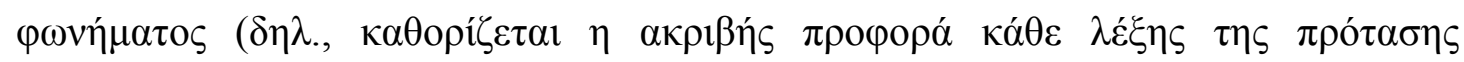

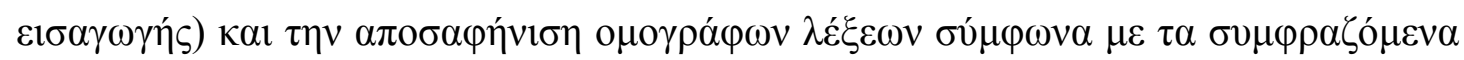

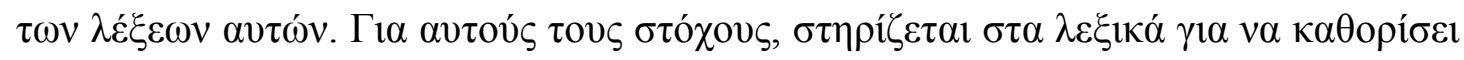

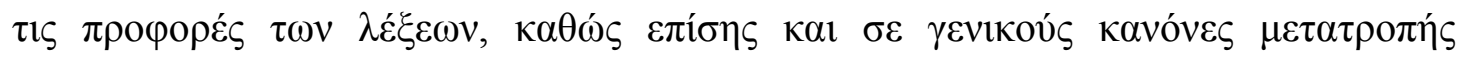




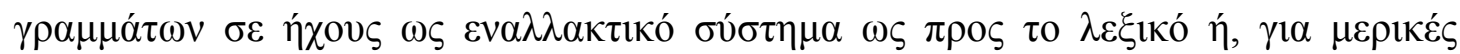

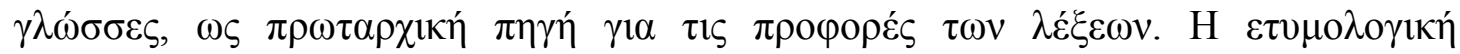

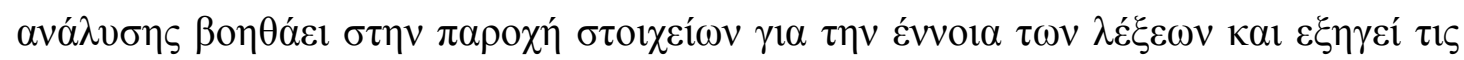

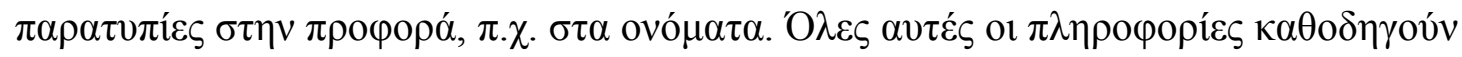
$\varepsilon \dot{\pi} \varepsilon 1 \tau \alpha \tau \eta v \pi \rho \circ \sigma \omega \delta 1 \alpha \kappa \eta ́ ~ \alpha v \alpha ́ \lambda v \sigma \eta$.

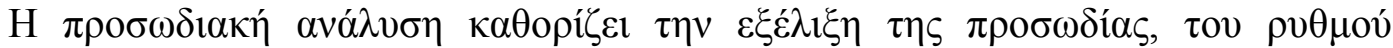

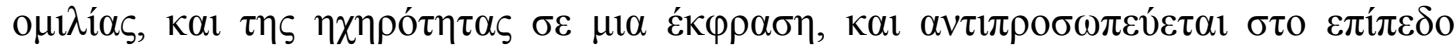

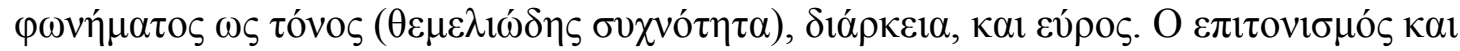

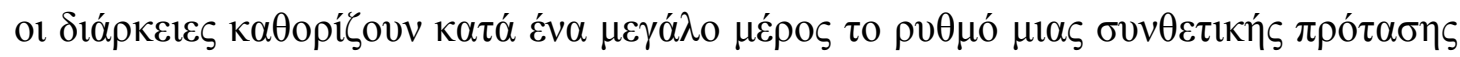

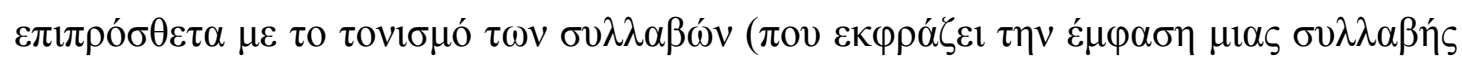

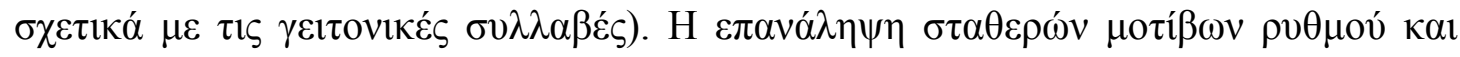

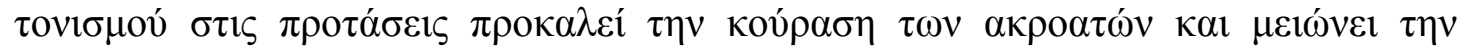

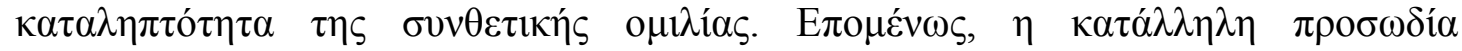

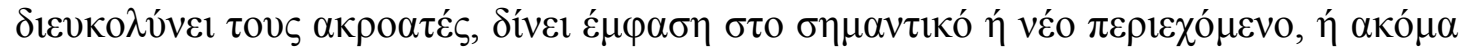

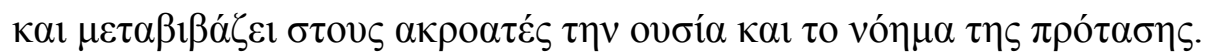

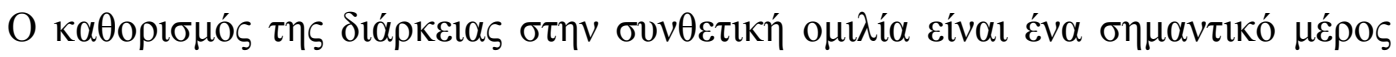

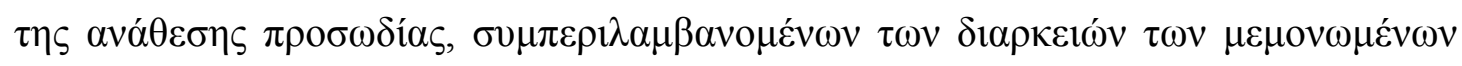

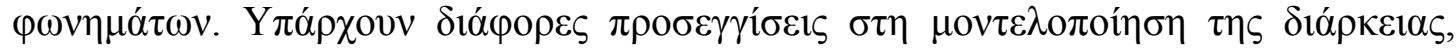

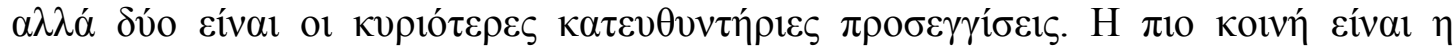

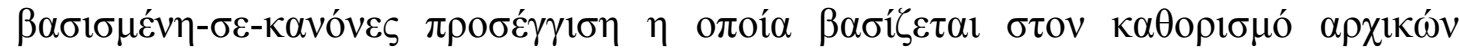

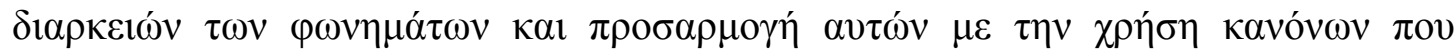

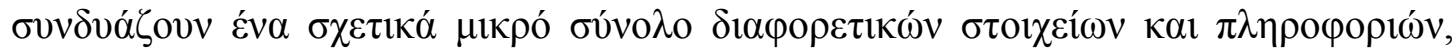

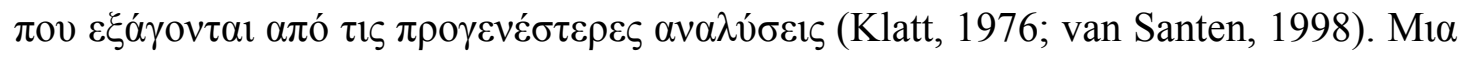

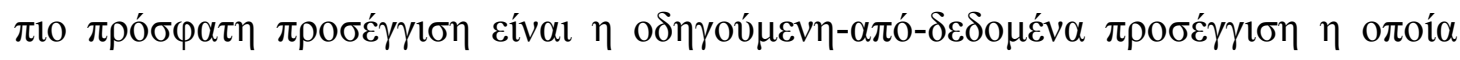

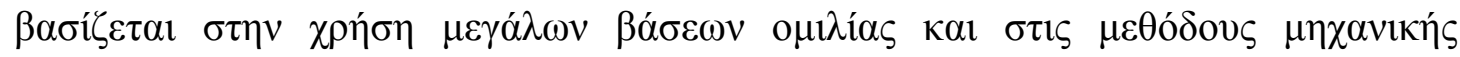

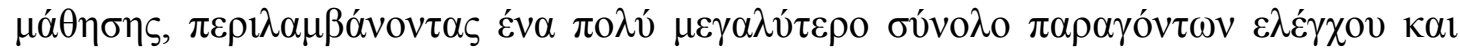

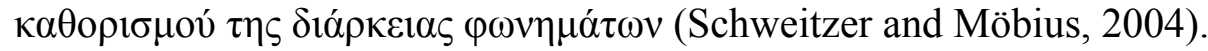

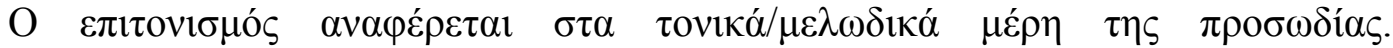

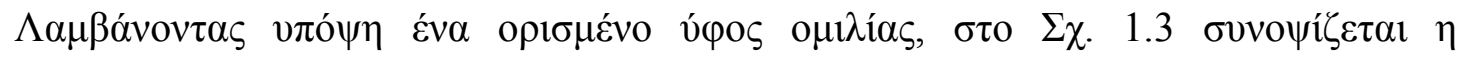

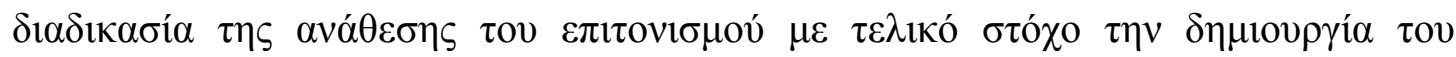

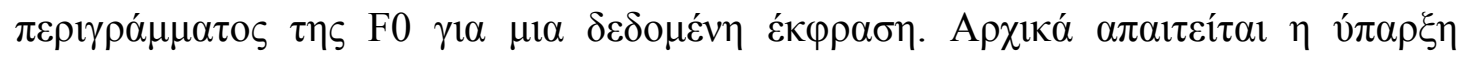

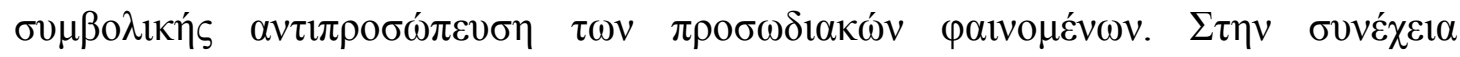

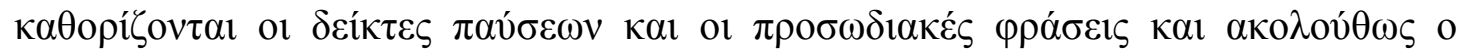

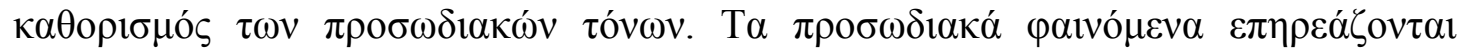




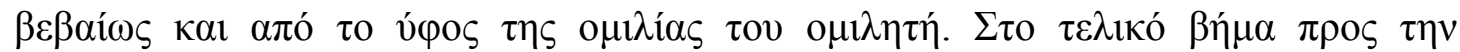

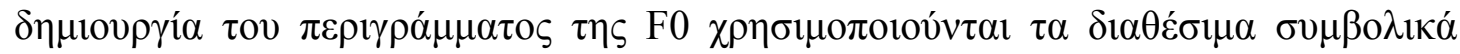

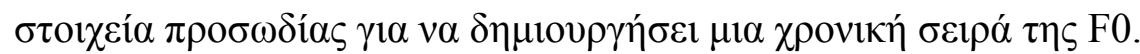

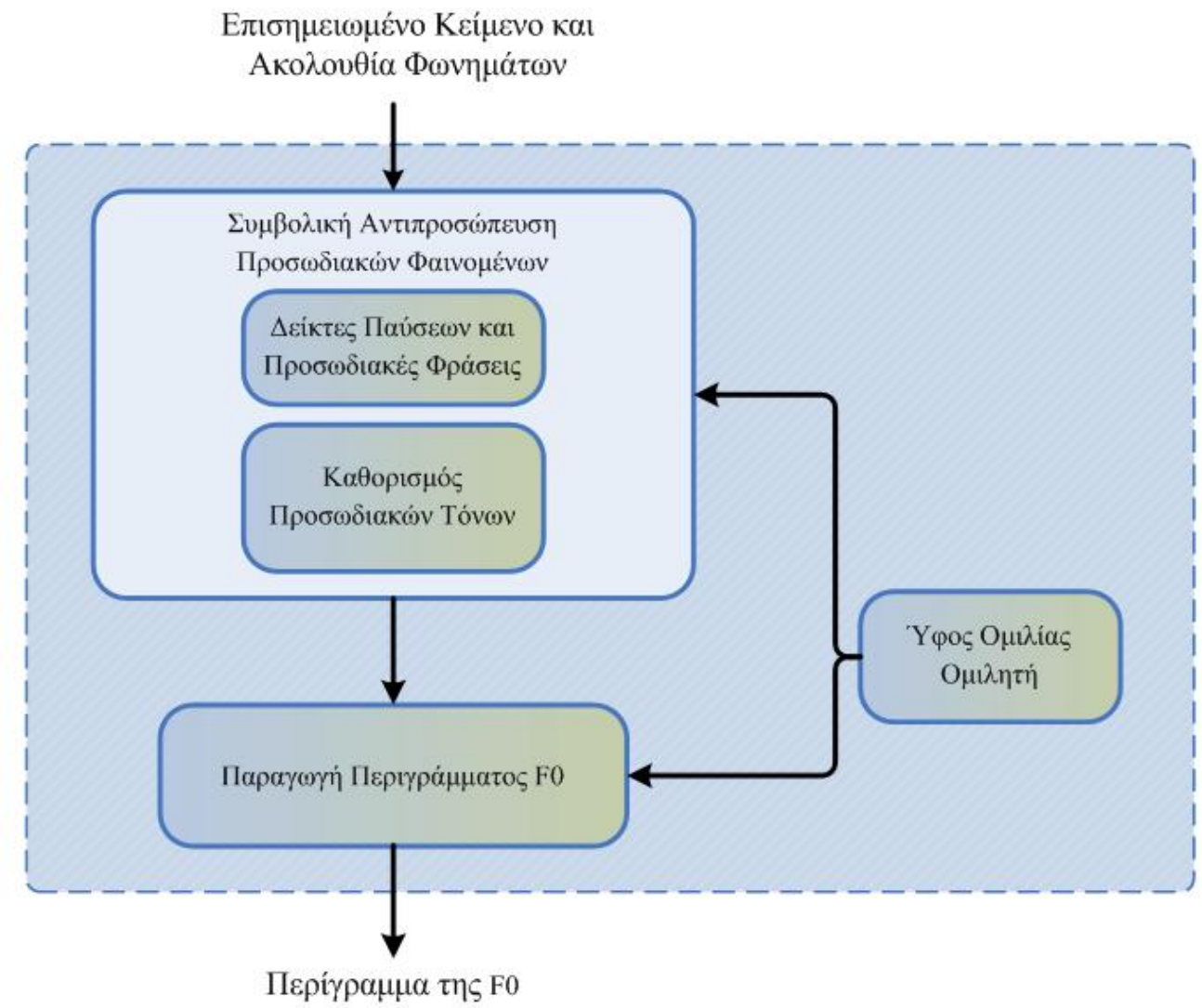

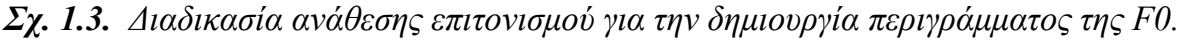

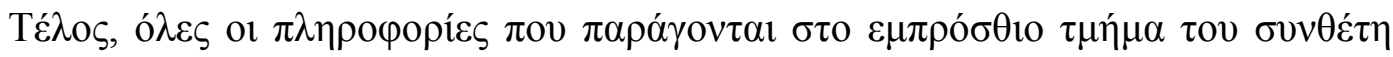

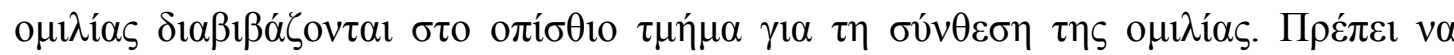

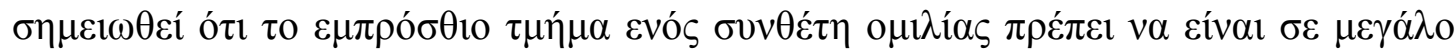

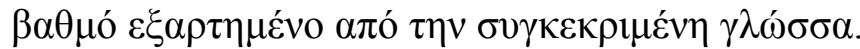

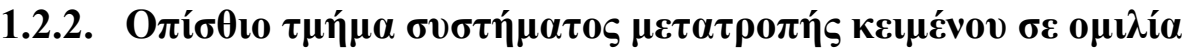

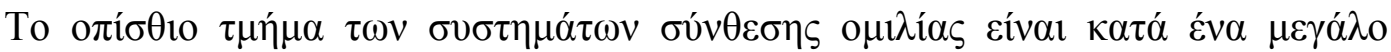

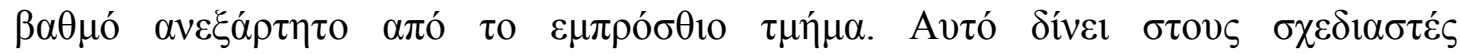

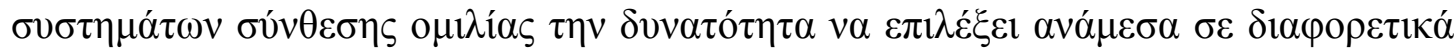

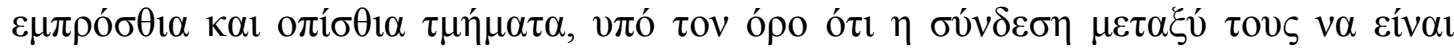




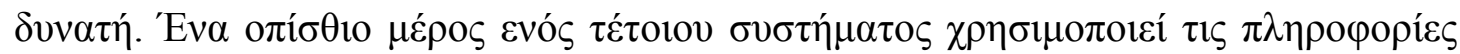

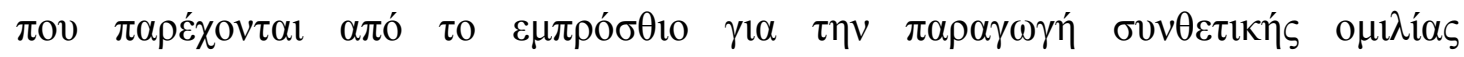

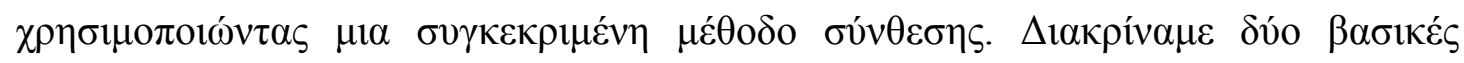

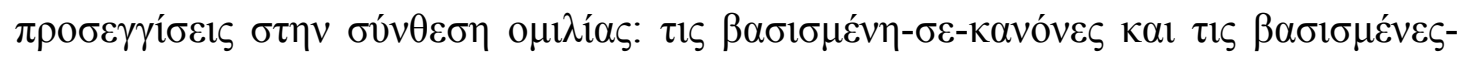

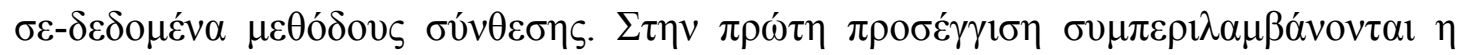

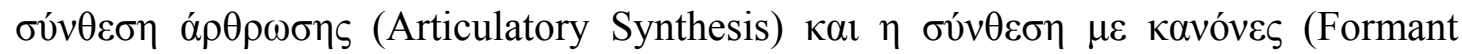

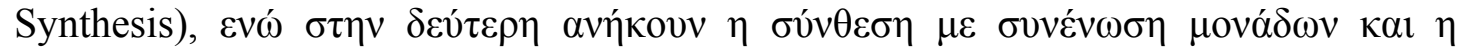

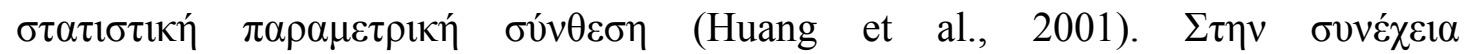

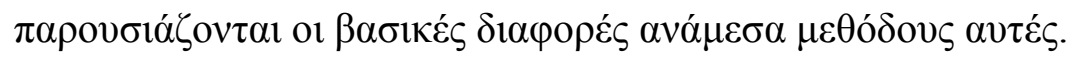

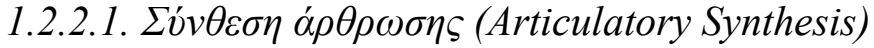

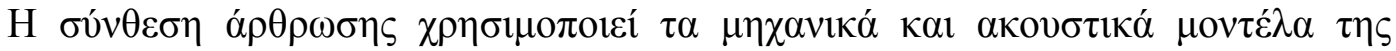

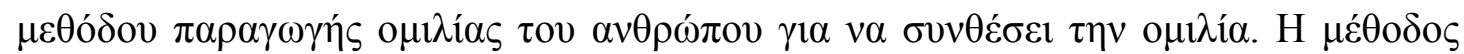

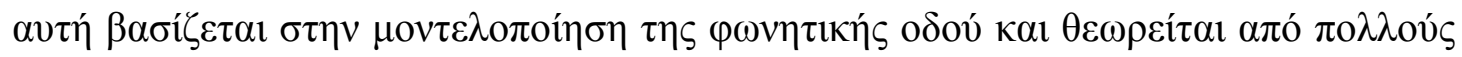

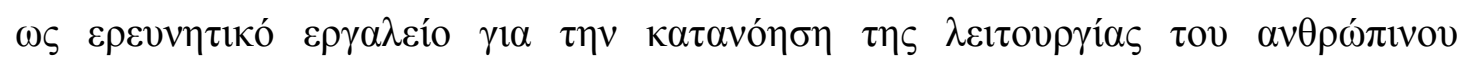

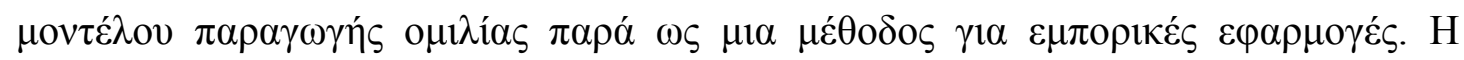

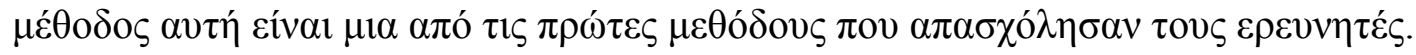

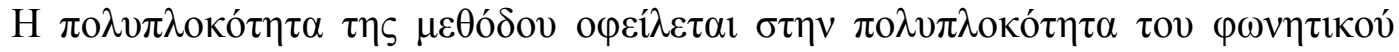

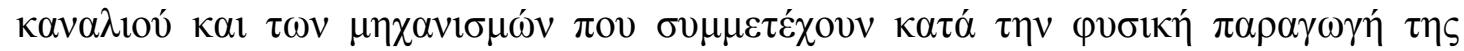

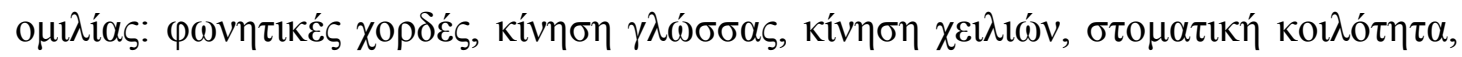

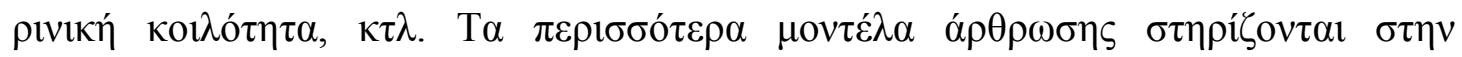

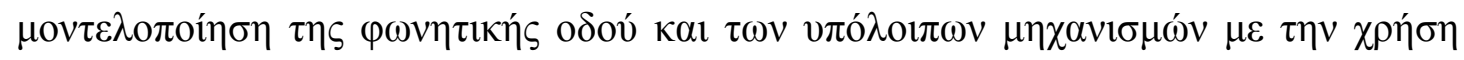

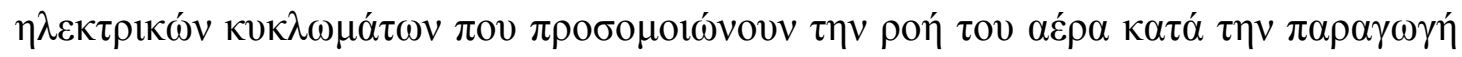

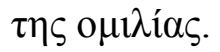

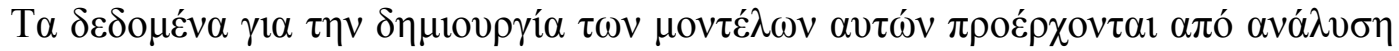

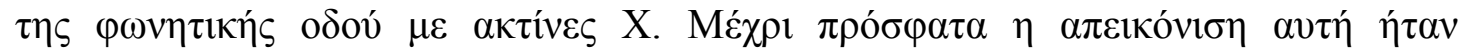

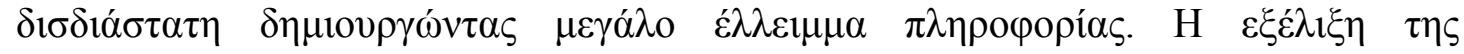

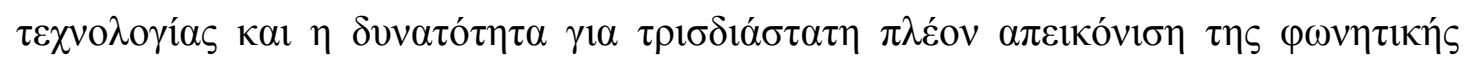

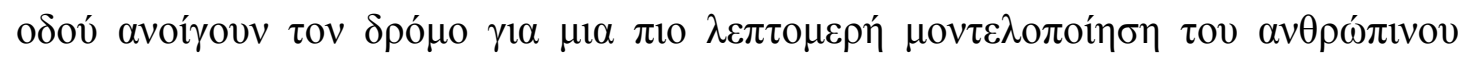

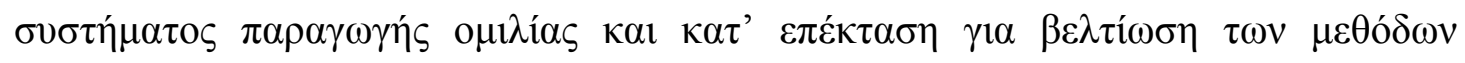
$\sigma u ́ v \theta \varepsilon \sigma \eta \varsigma \mu \varepsilon \alpha \dot{\alpha} \rho \theta \rho \omega \sigma \eta$. 


\subsubsection{2. $\Sigma v ́ v \theta \varepsilon \sigma \eta \mu \varepsilon \kappa \alpha v o ́ v \varepsilon \varsigma$ (Formant Synthesis)}

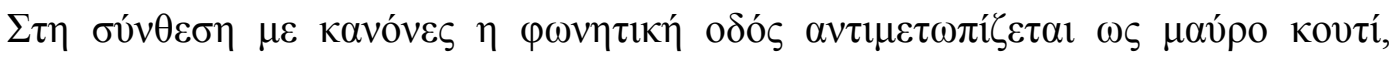

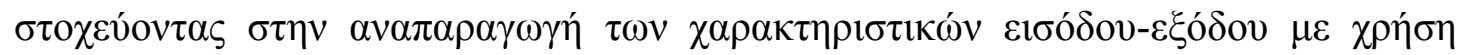

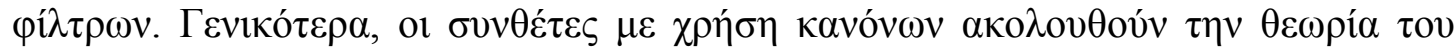

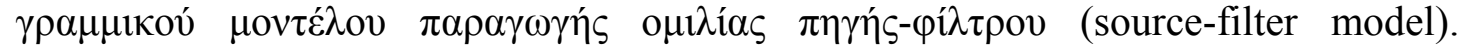

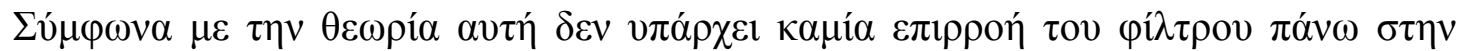

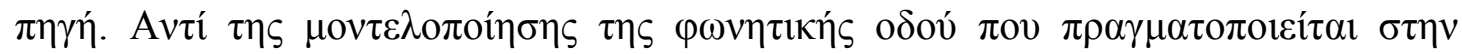

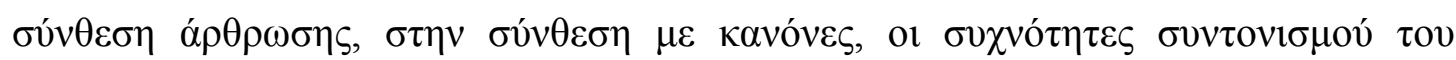

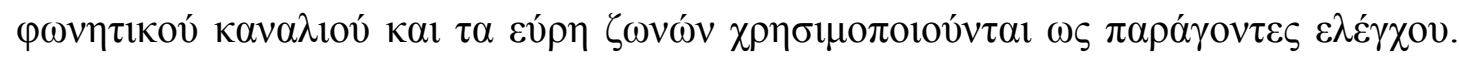

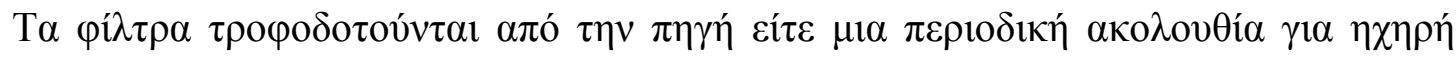

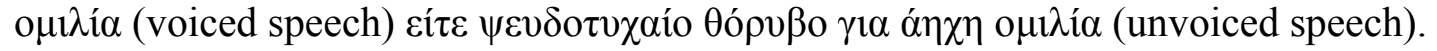

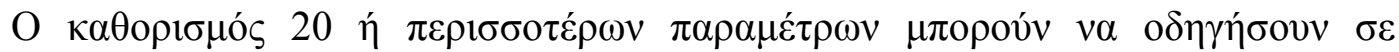

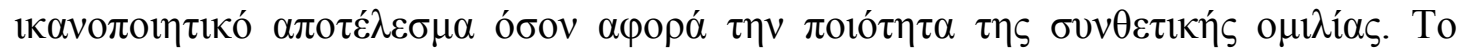

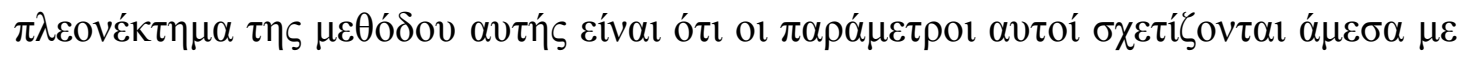

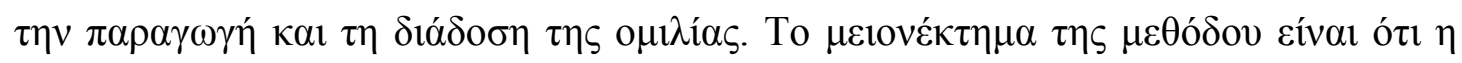

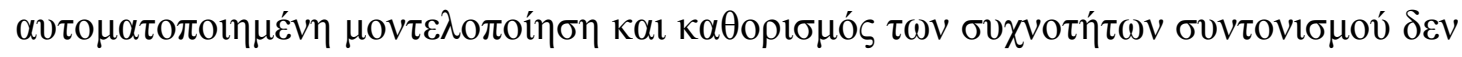

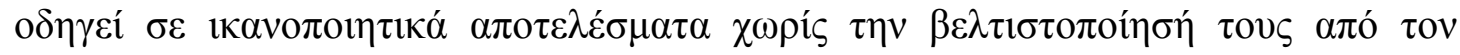
óv$\theta \rho \omega \pi \mathrm{o}$.

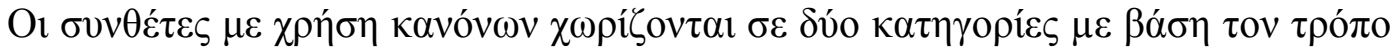

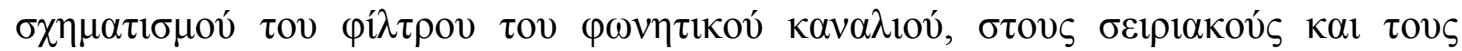

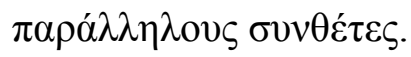

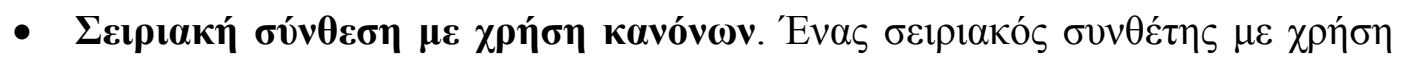

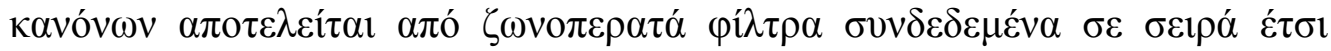

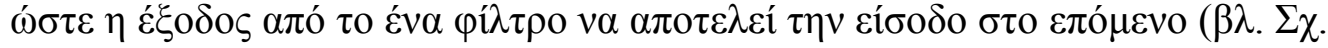

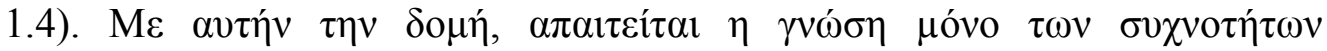

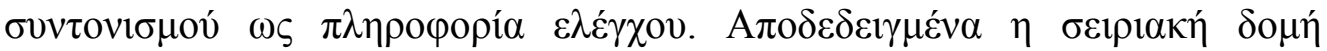

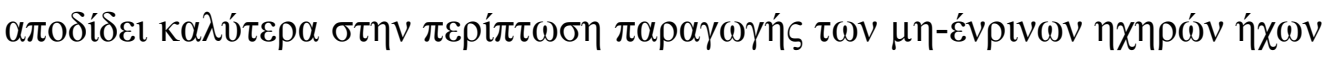

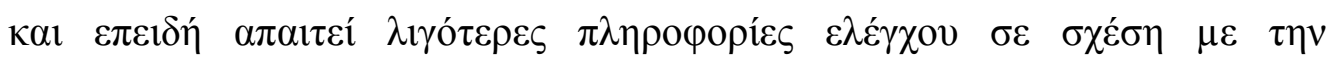

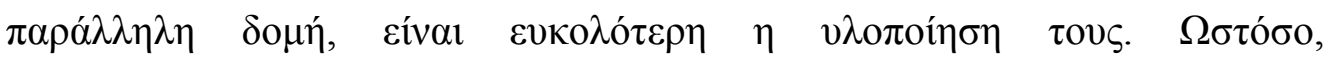

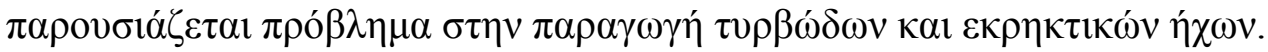




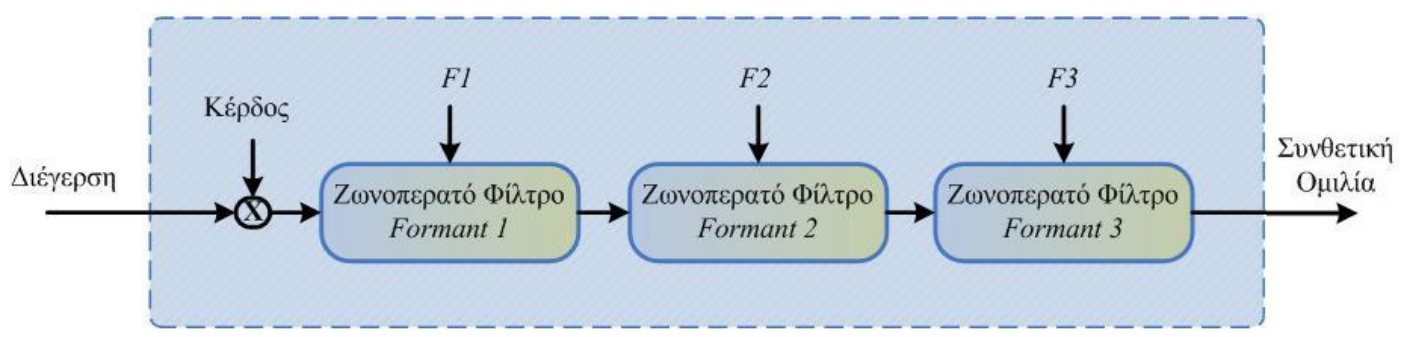

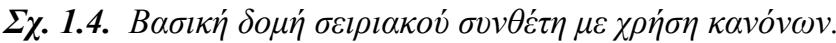

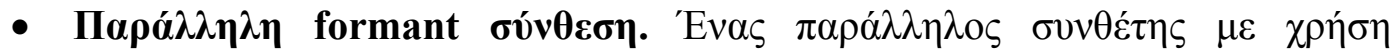

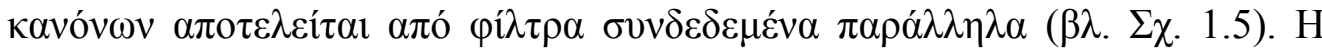

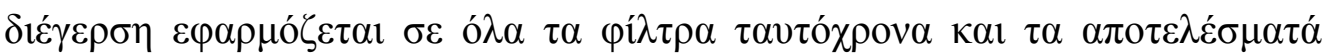

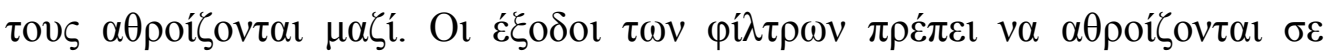

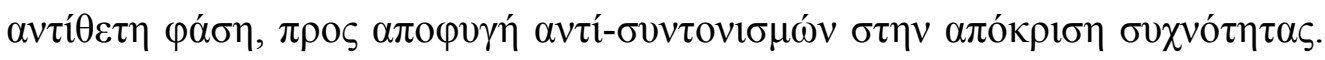

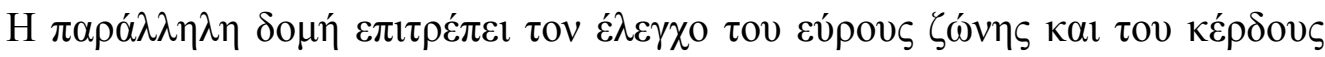

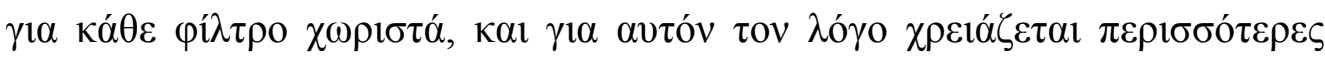

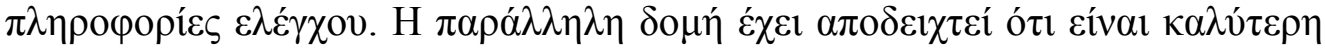

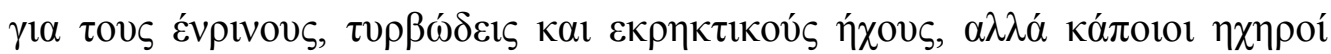

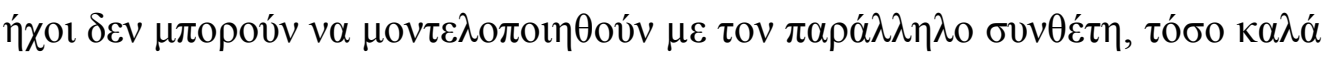

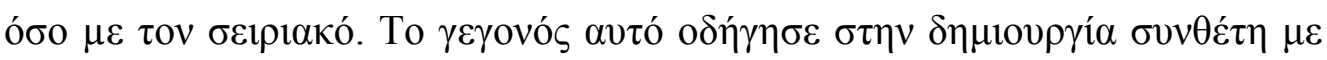

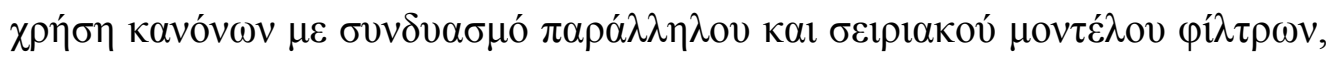

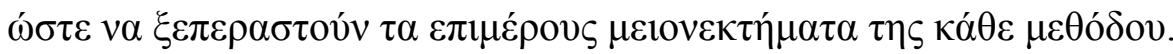




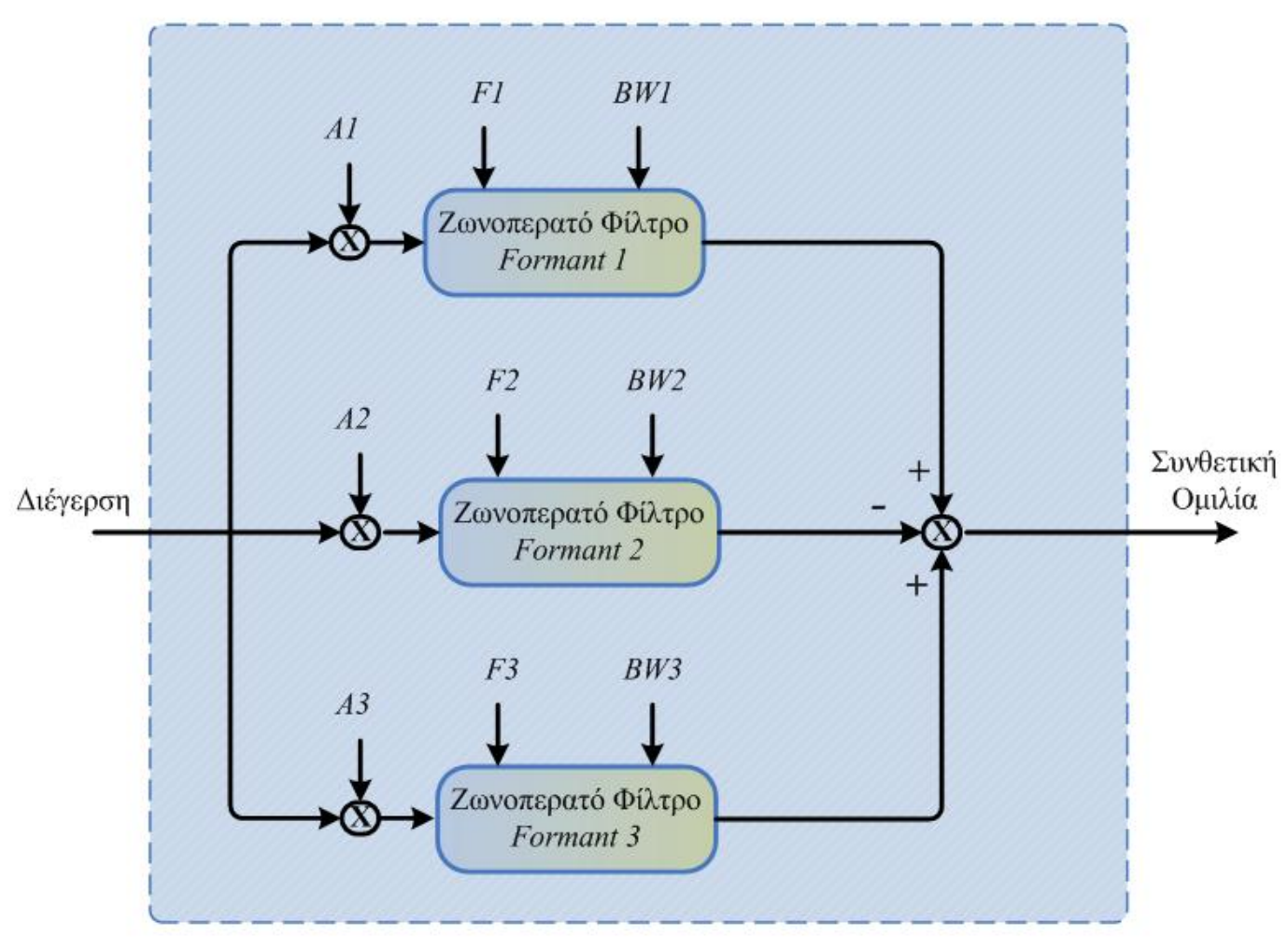

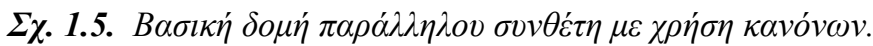

\subsubsection{3. $\Sigma v v v \theta \varepsilon \sigma \eta \mu \varepsilon \sigma v v \varepsilon ́ v \omega \sigma \eta \mu o v \alpha ́ \delta \omega v$ (Concatenative Synthesis)}

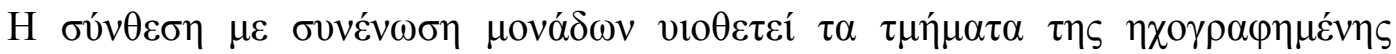

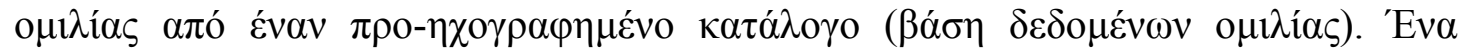

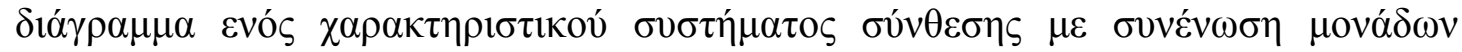

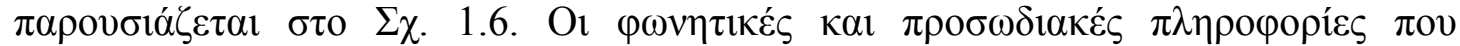

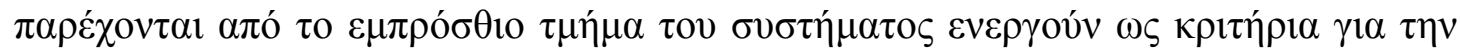

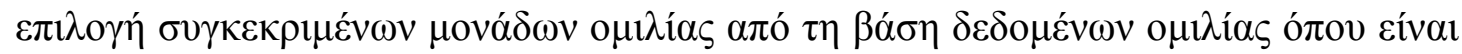

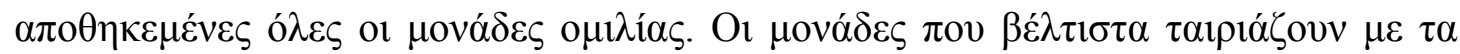

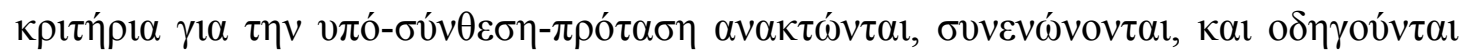

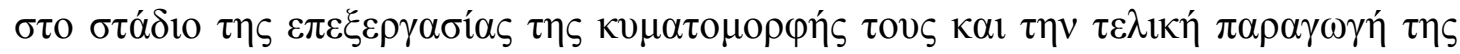

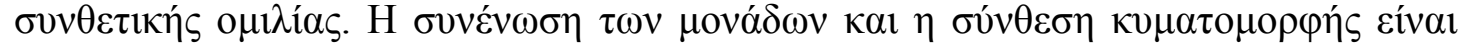

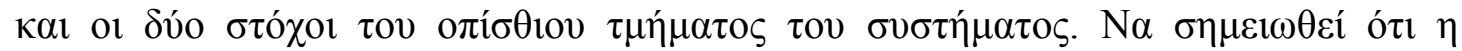

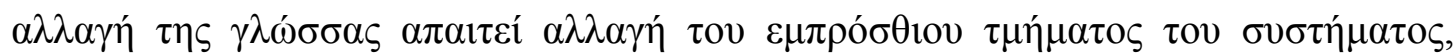

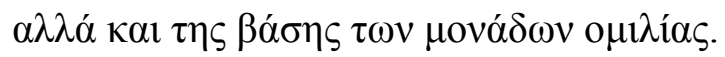




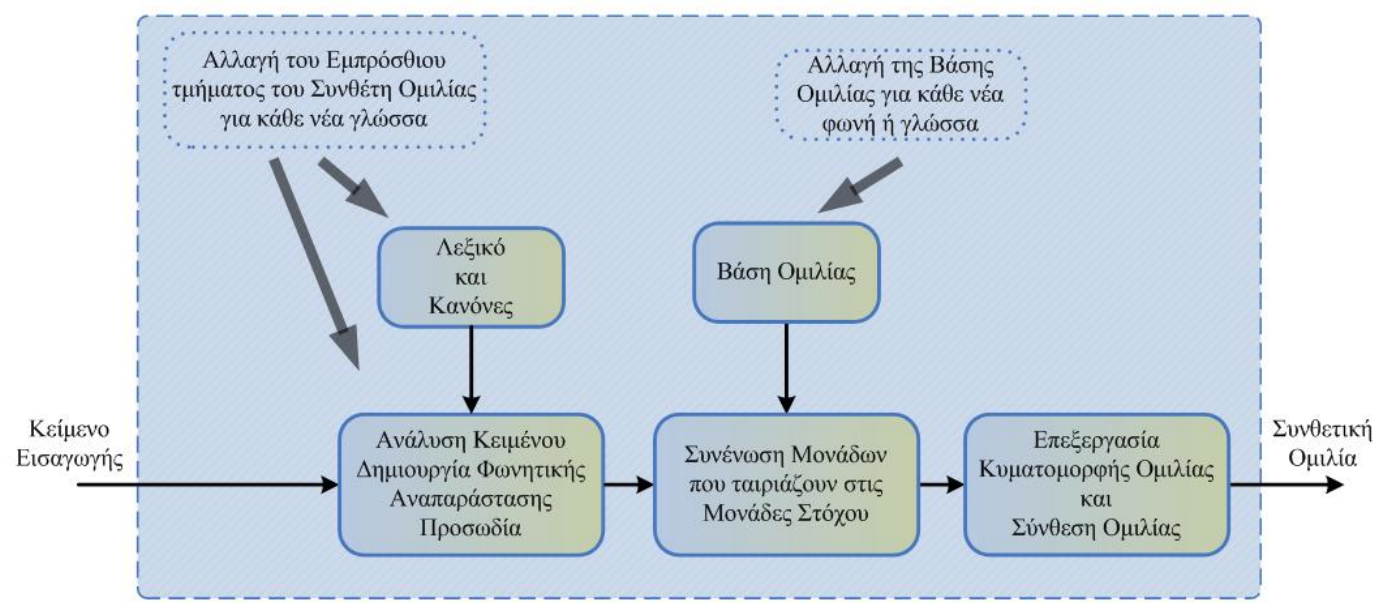

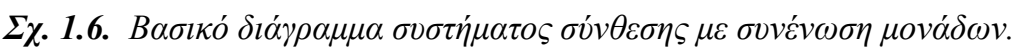

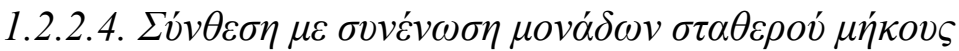

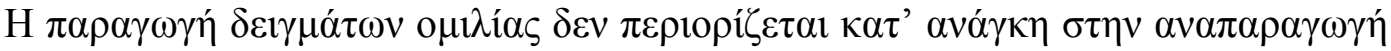

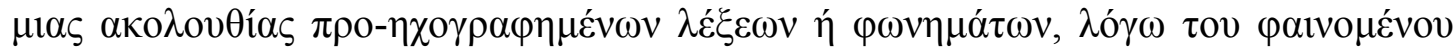

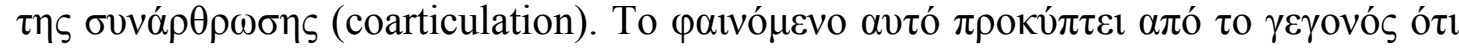

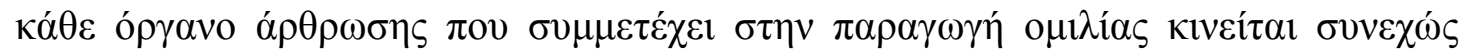

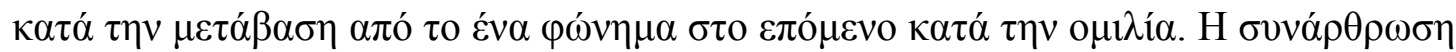

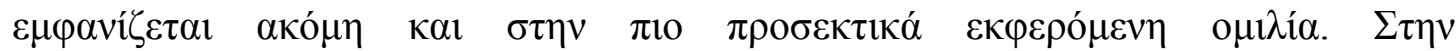

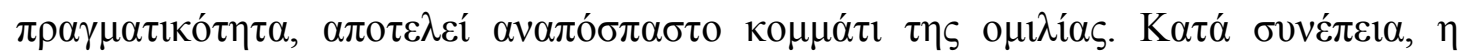

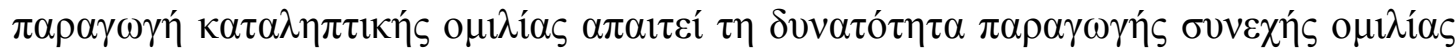
$\mu \varepsilon \sigma \omega \sigma \tau \eta ́ ~ \sigma v v \alpha ́ \rho \theta \rho \omega \sigma \eta$.

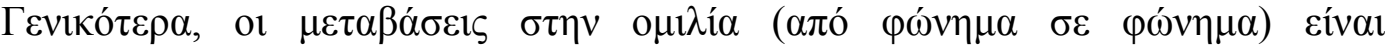

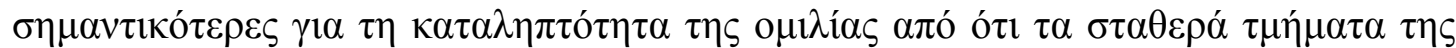

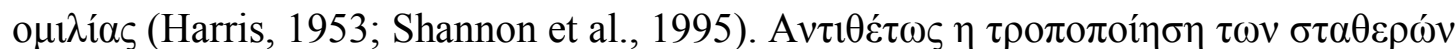

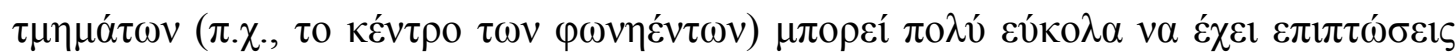

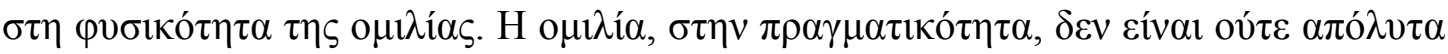

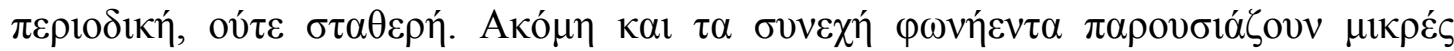

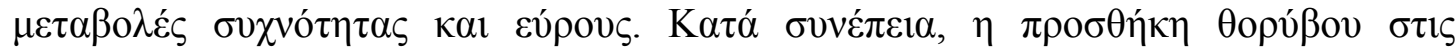

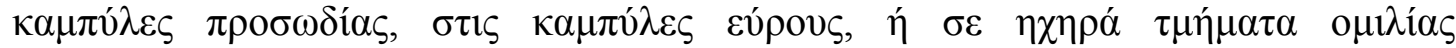

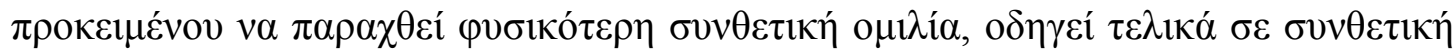

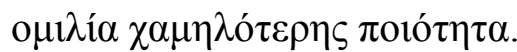




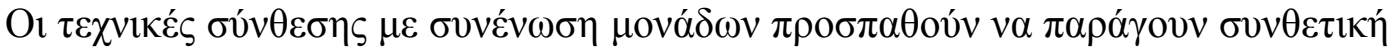

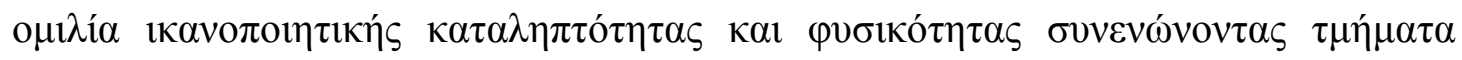

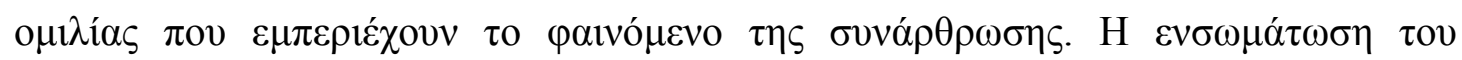

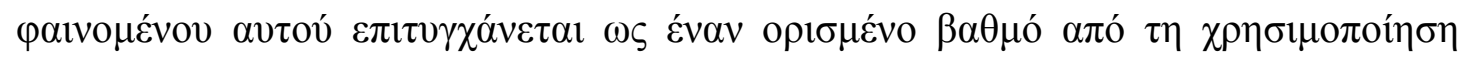

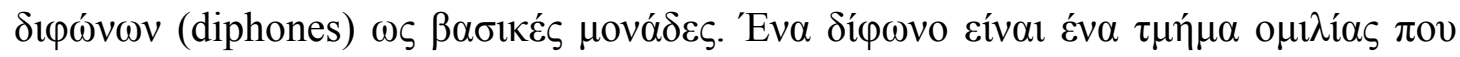

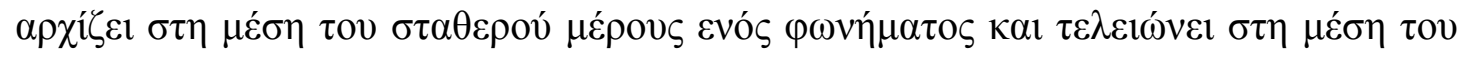

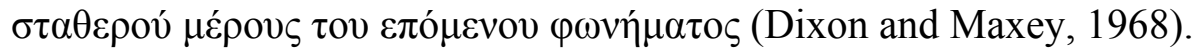

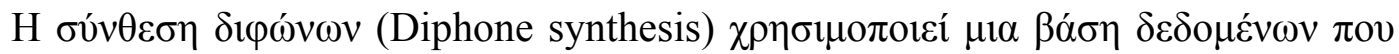

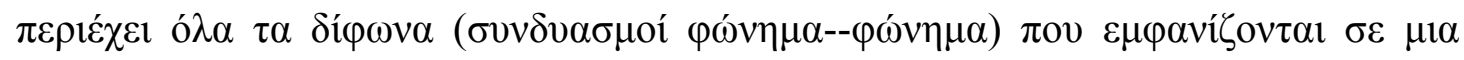

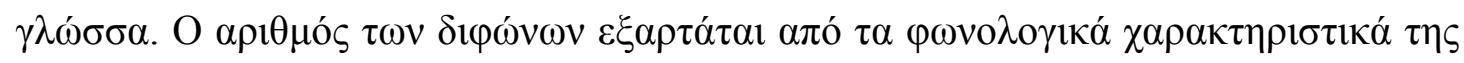

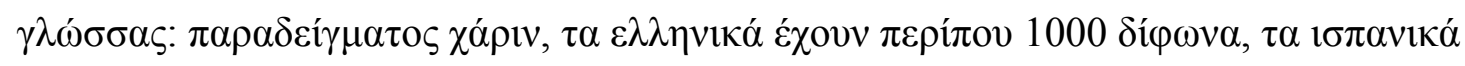

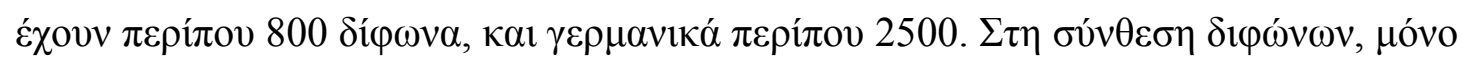

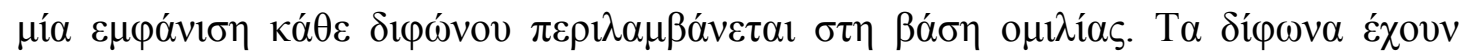

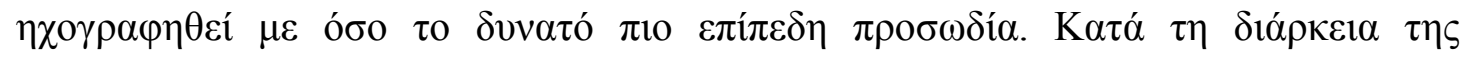

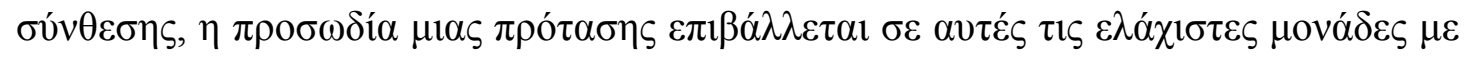

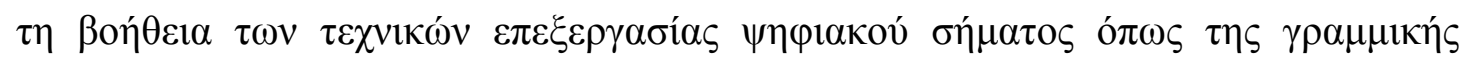

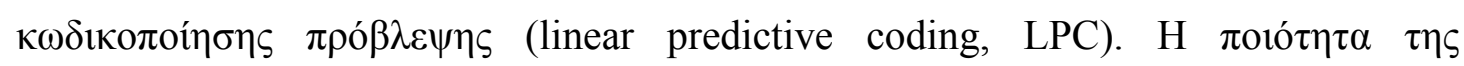

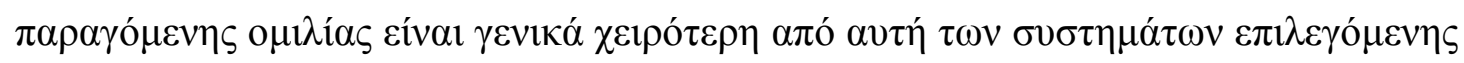

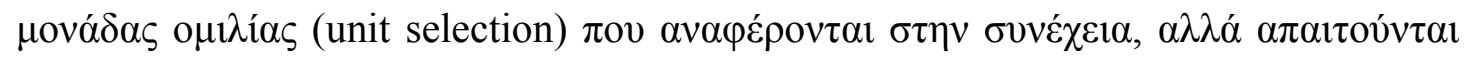

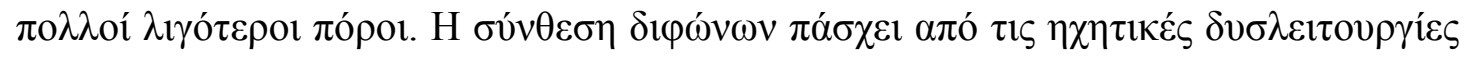

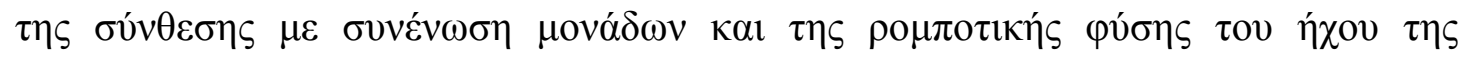

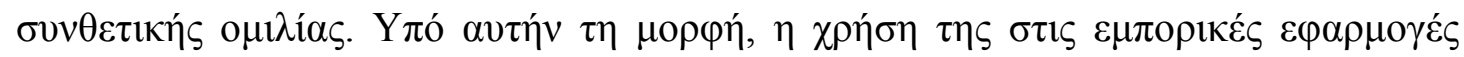

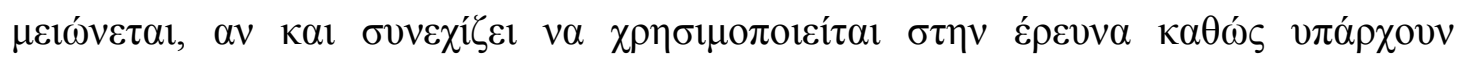

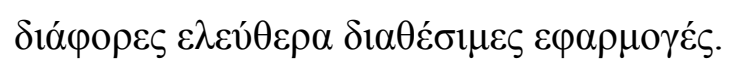

\subsubsection{5. $\Sigma v ́ v \theta \varepsilon \sigma \eta \mu \varepsilon \varepsilon \pi \imath \lambda o \gamma \eta \dot{~ \mu o v \alpha ́ \delta \omega v ~(U n i t-S e l e c t i o n ~ S y n t h e s i s) ~}$}

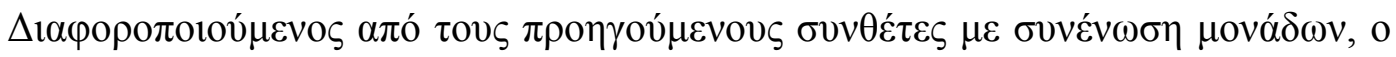

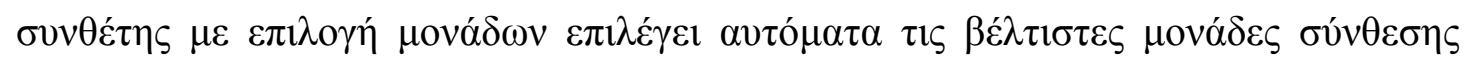

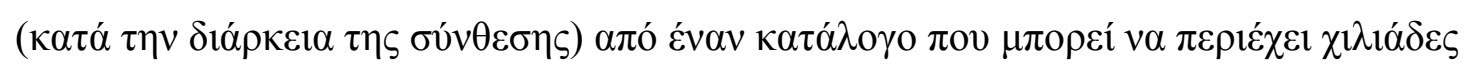

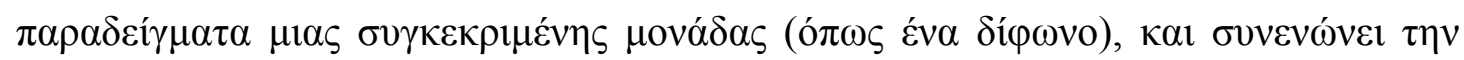

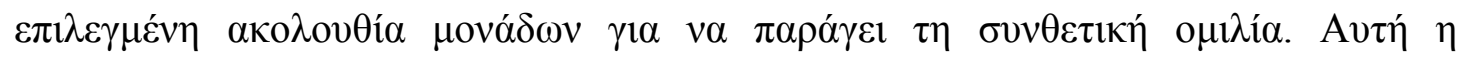

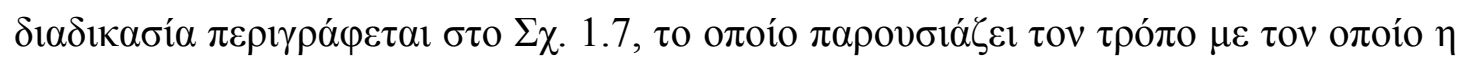




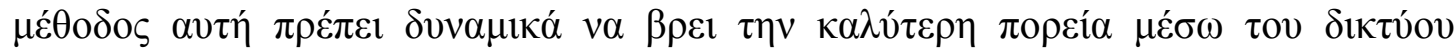

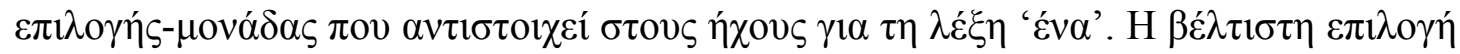

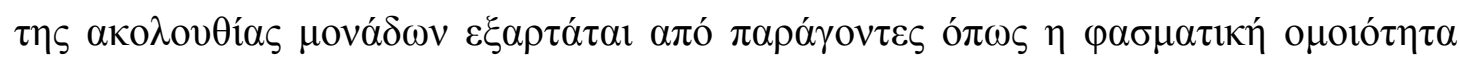

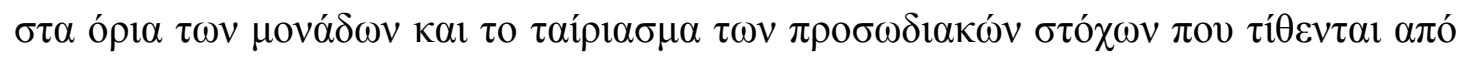

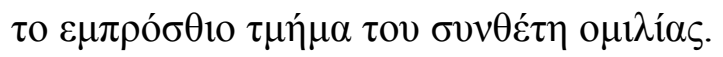

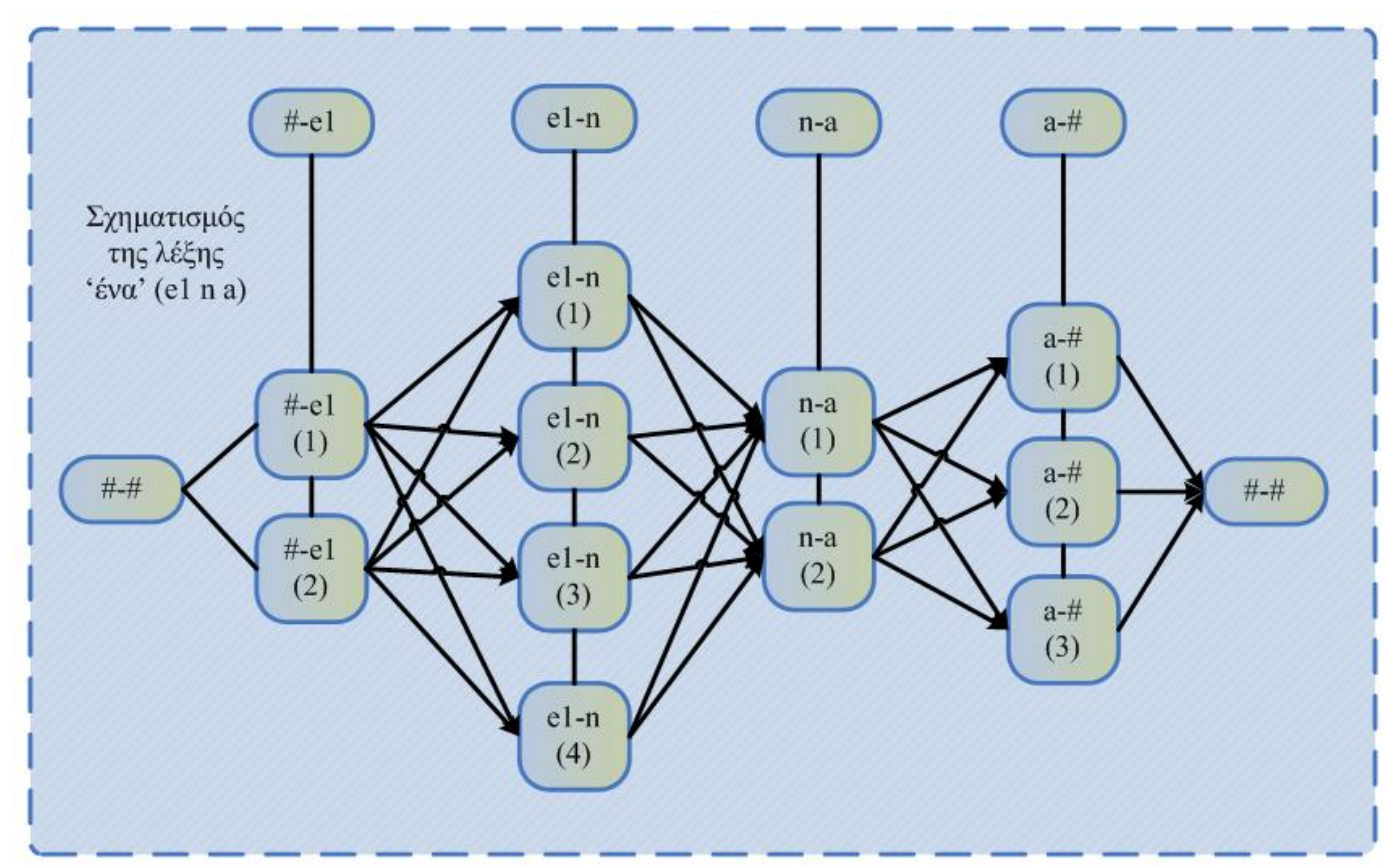

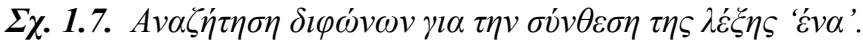

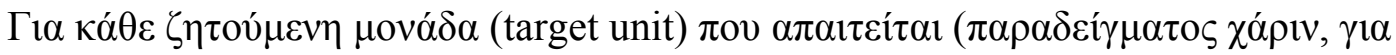

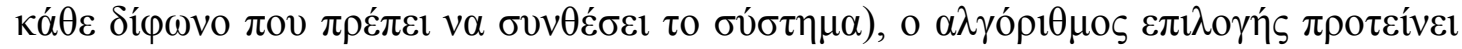

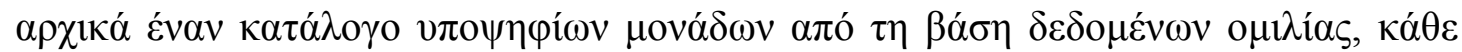

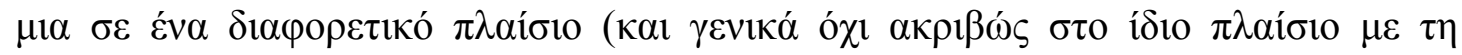

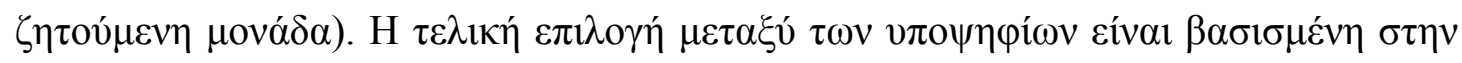

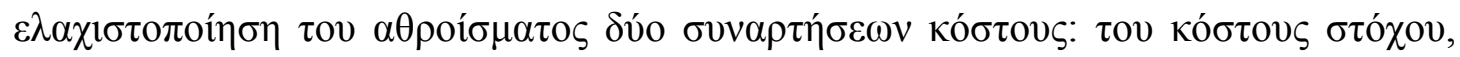

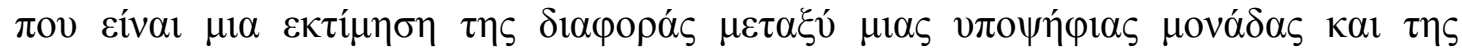

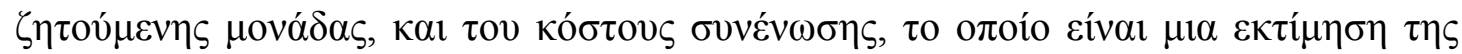

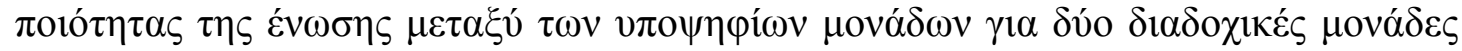

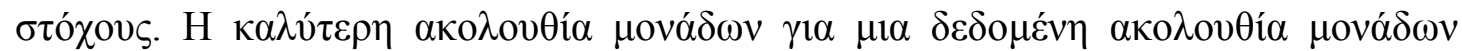

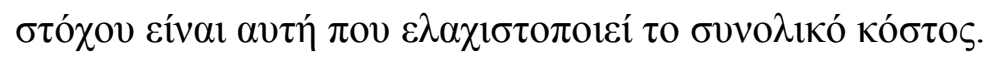

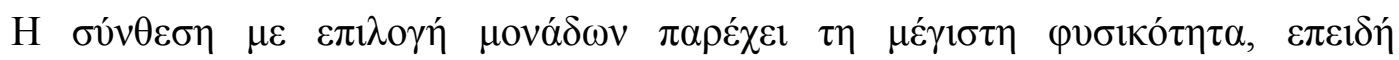

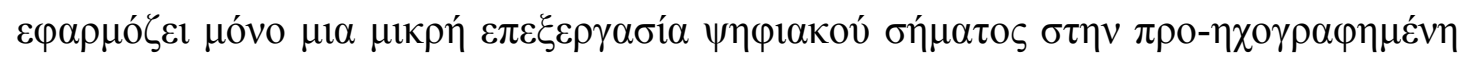




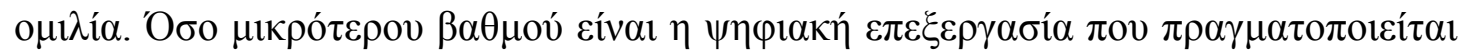

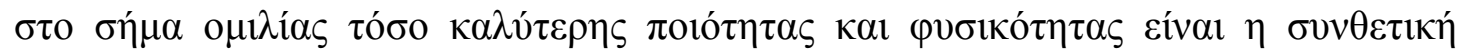

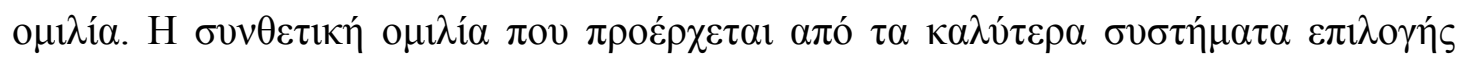

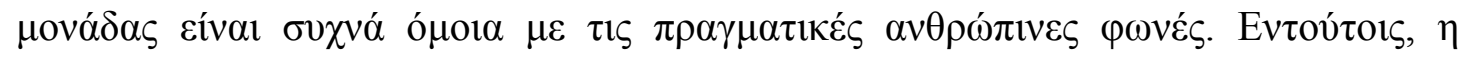

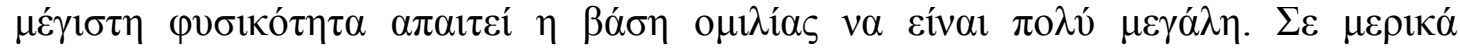

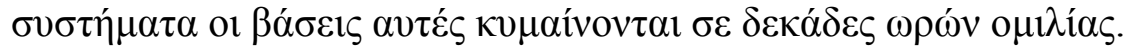

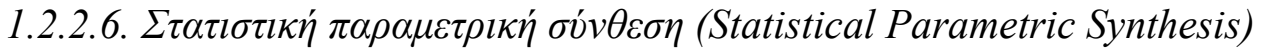

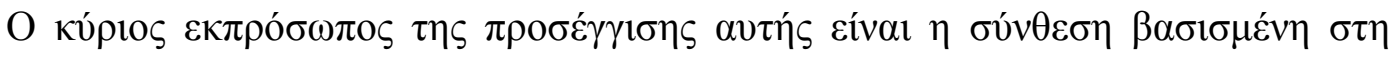

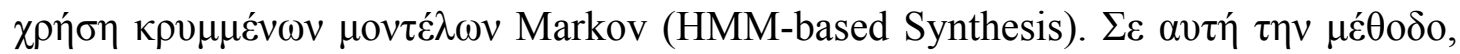

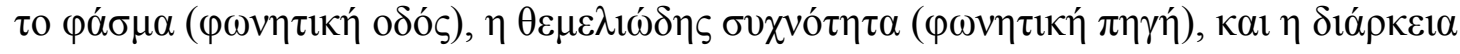

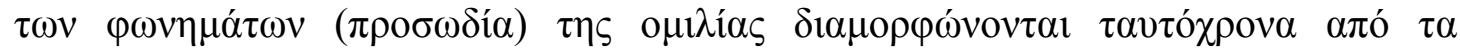

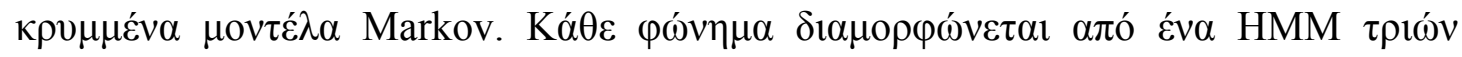

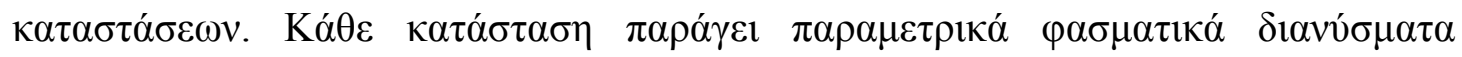

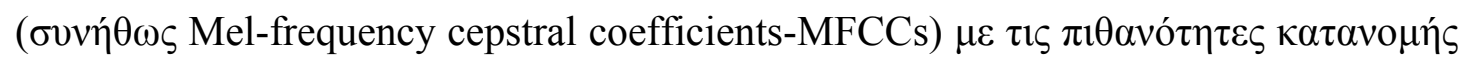

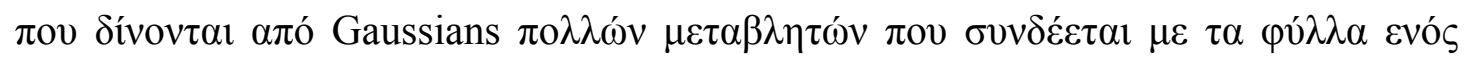

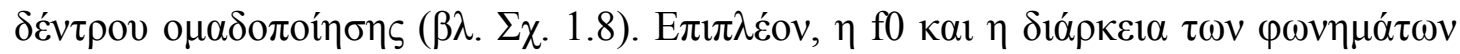

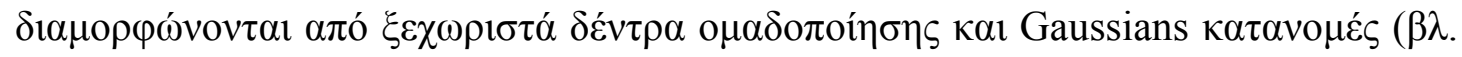
$\Sigma \chi .1 .8)$.

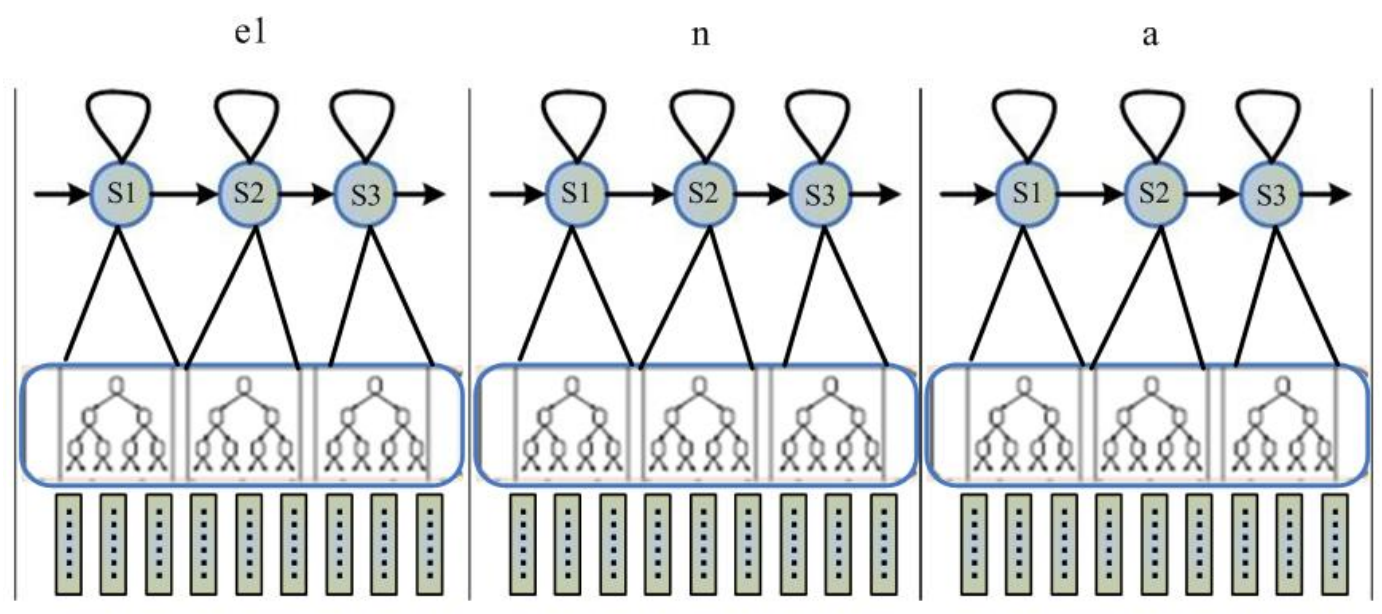

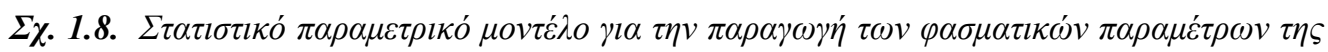
$\lambda \dot{\varepsilon} \xi \eta \varsigma$ ' $\varepsilon \alpha$ '. 


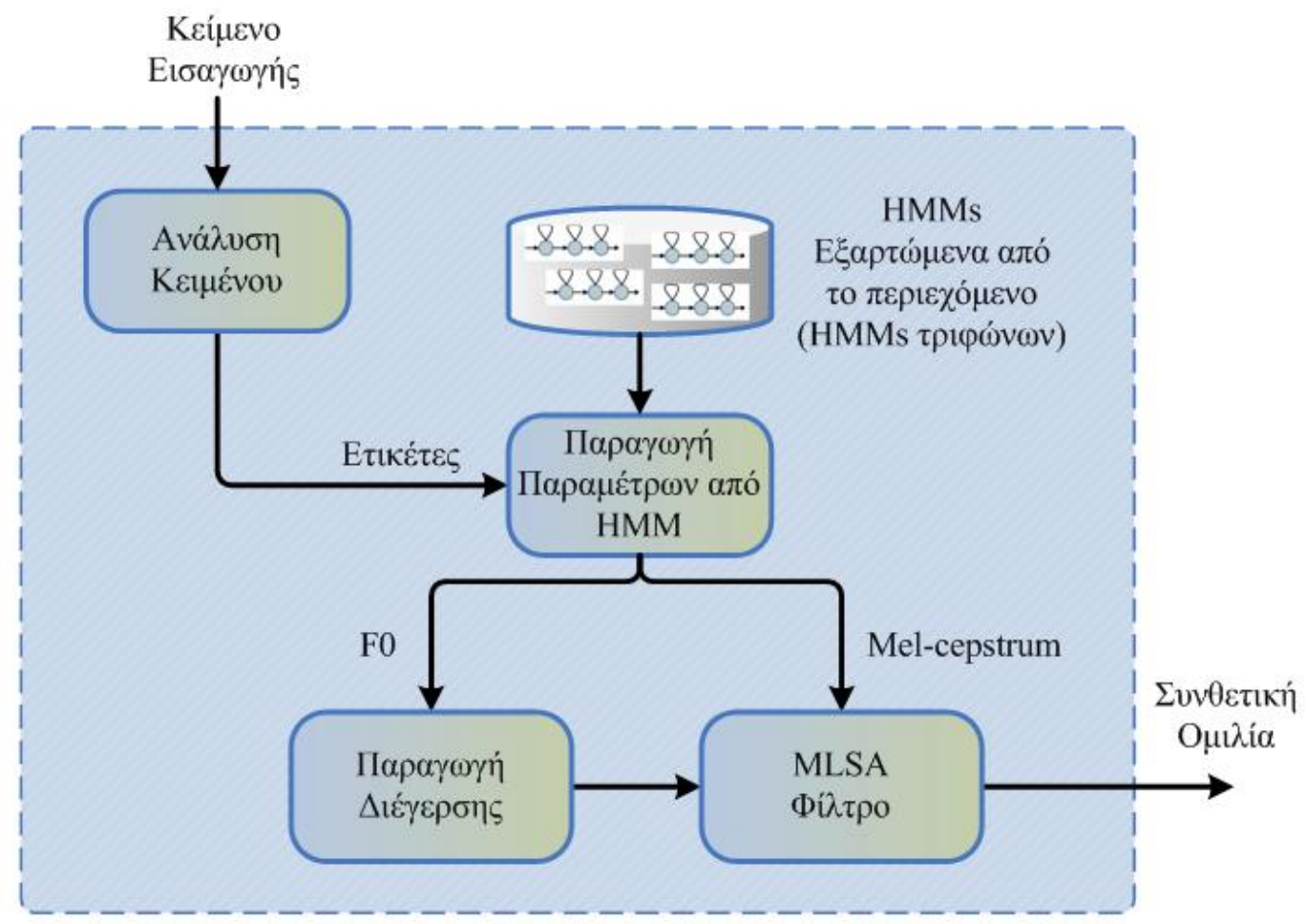

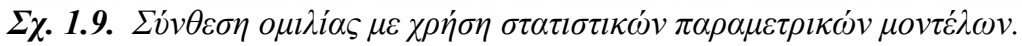

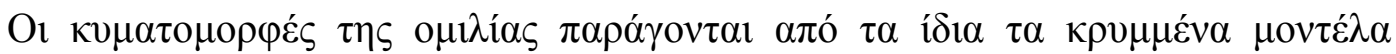

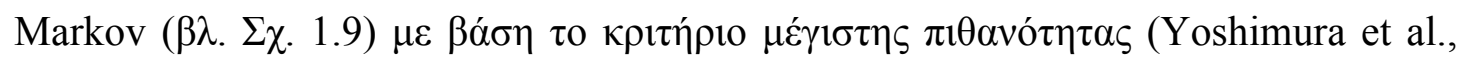

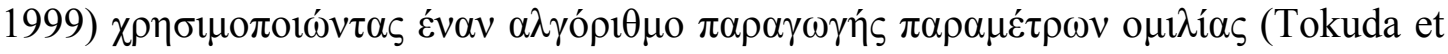

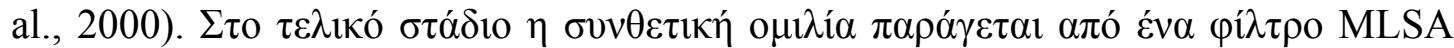

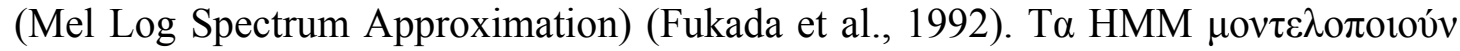

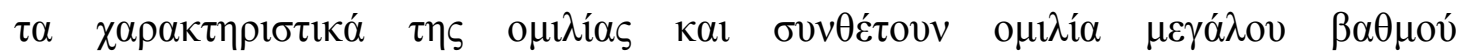

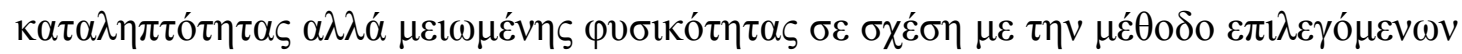

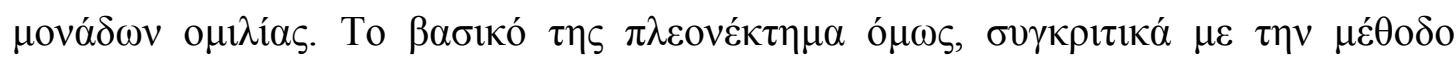

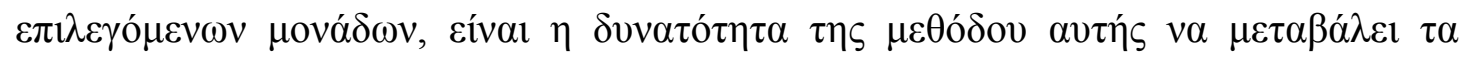

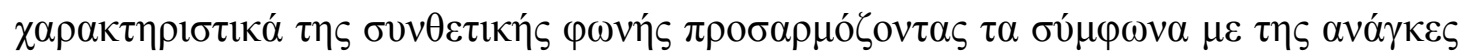

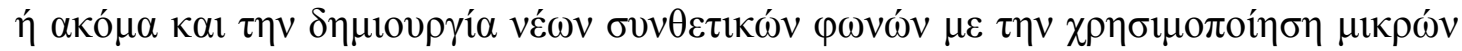

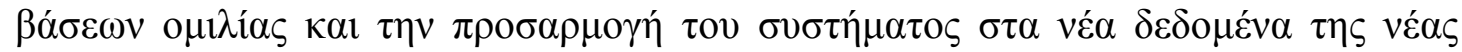

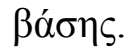




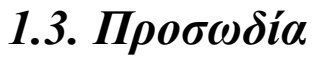

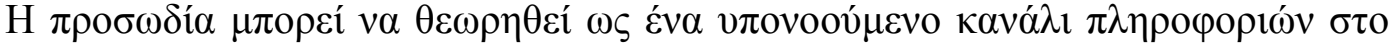

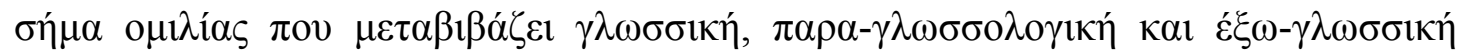

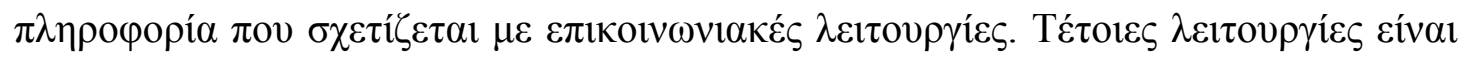

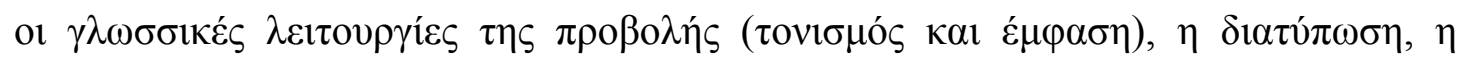

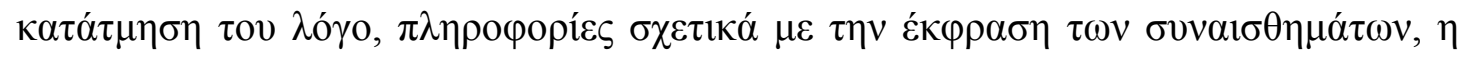

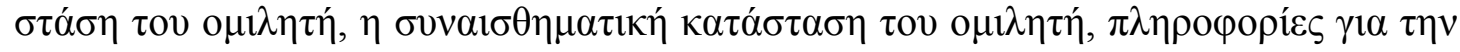

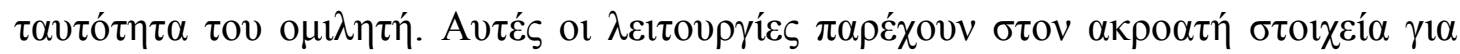

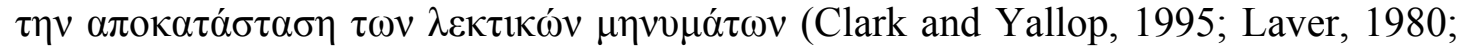

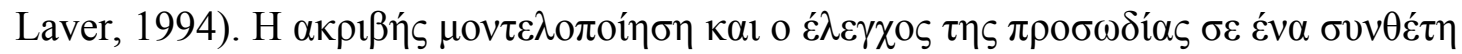

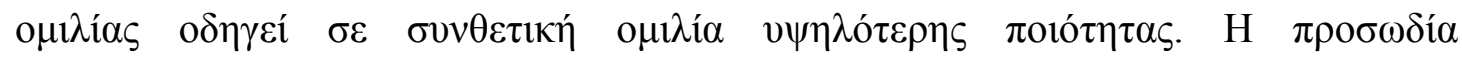

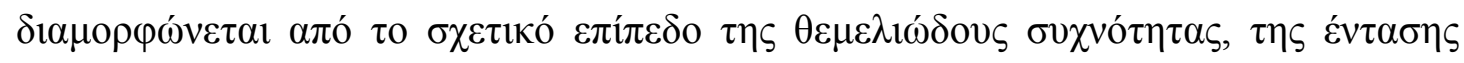

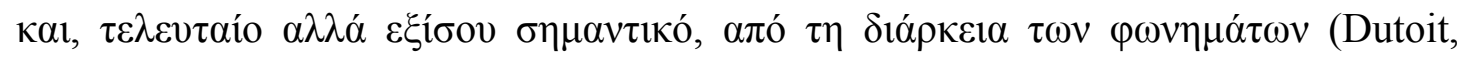
1997; Furui, 2000).

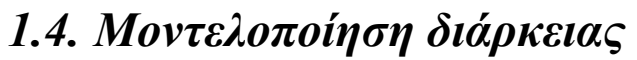

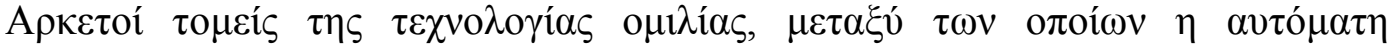

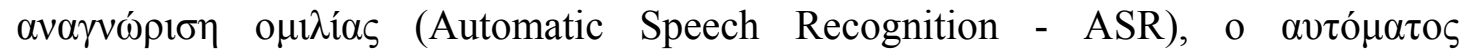

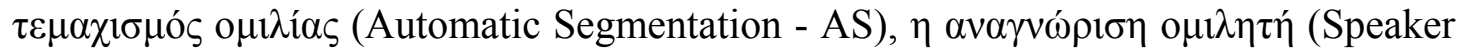

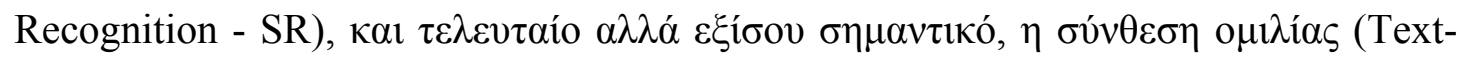

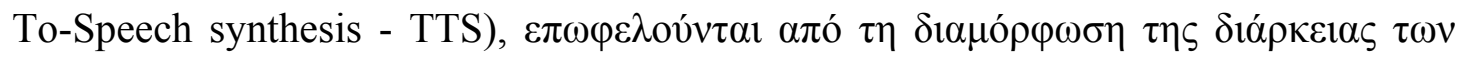

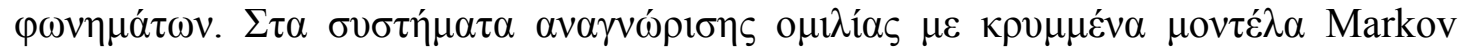

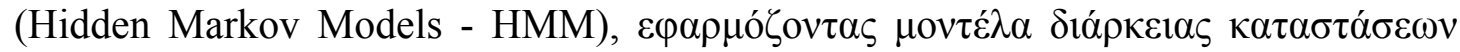

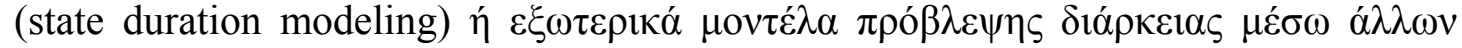

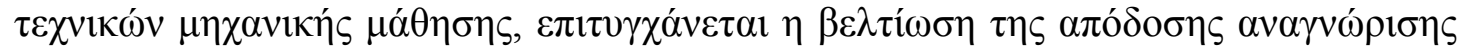
opı入ías (Anastasakos et al, 1995; Bourlard et al, 1996; Jennequin and Gauvian, 2007; Levinson, 1986; Mitchell et al,1995; Pols et al, 1996; Pylkkonen and Kurimo, 2004).

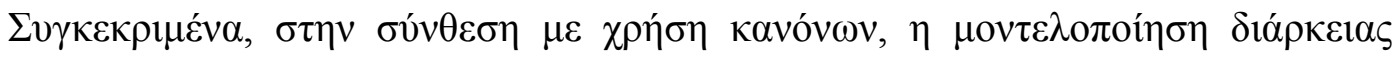

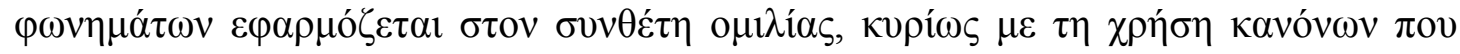

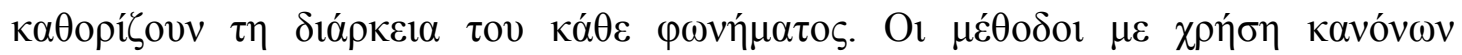




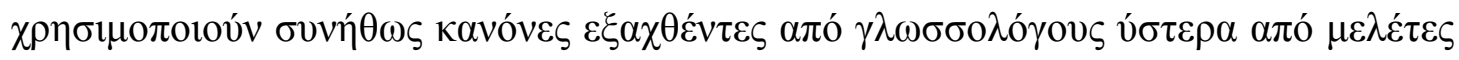

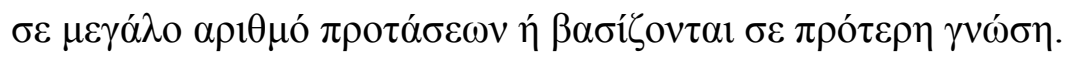

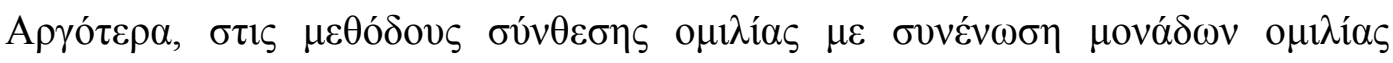

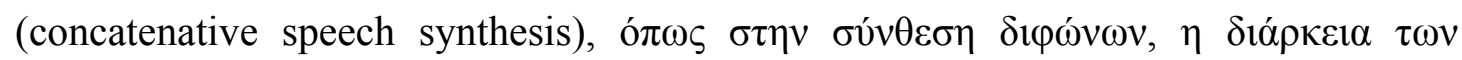

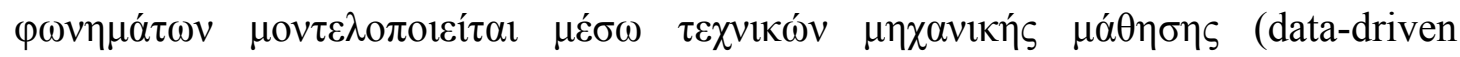

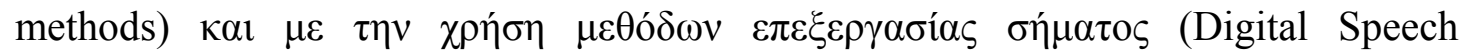

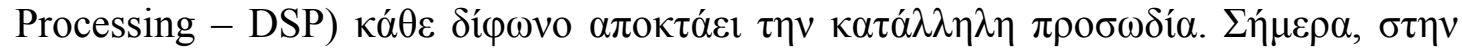

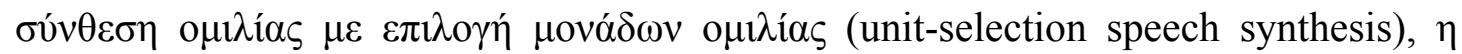

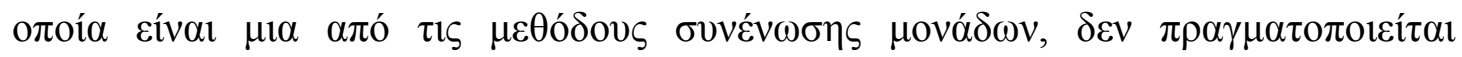

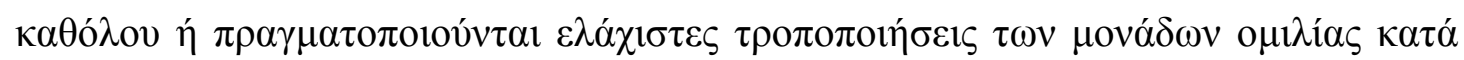

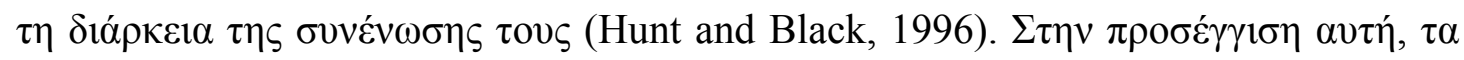

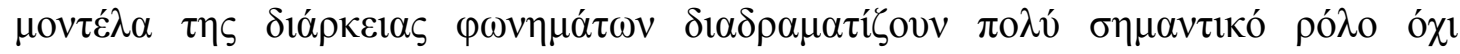

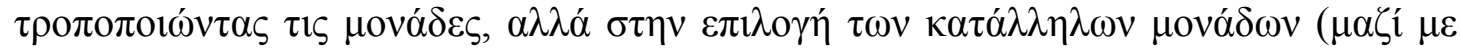

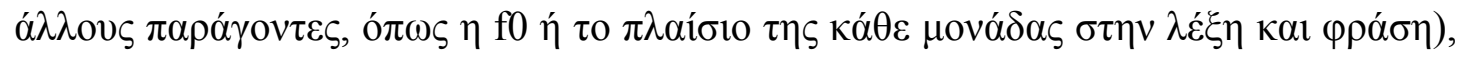

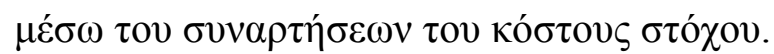

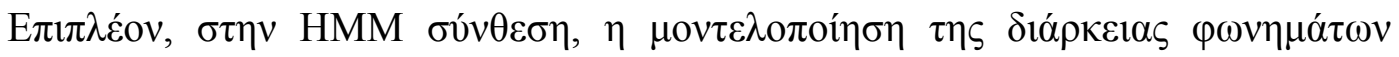

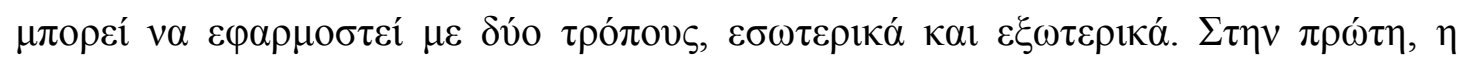

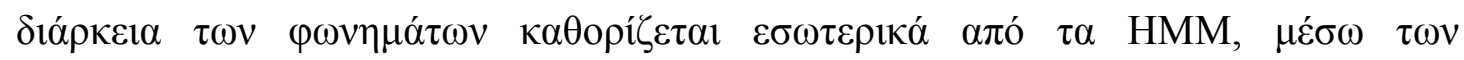

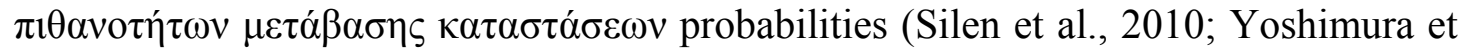

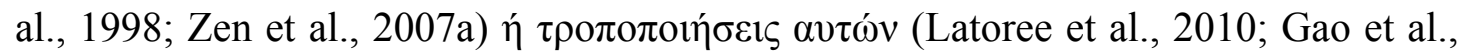

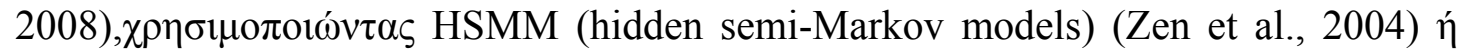

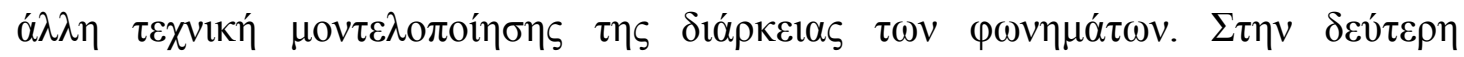

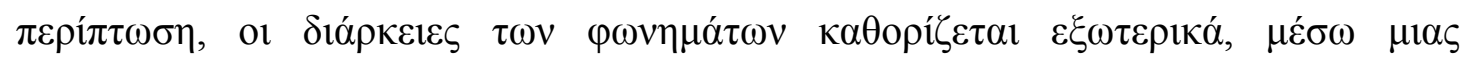

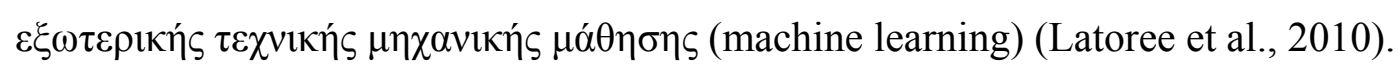

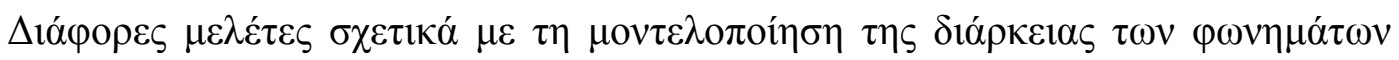
(Bartkova and Sorin, 1987; Bell et al., 2003; Carlson and Granstrom, 1986; Chung, 2002; Crustal and House, 1988; Epitropakis et al., 1993; Goubanova and King, 2008; Gregory et al., 2001; Iwahashi and Sagisaka, 2000; Klatt, 1976; Kohler, 1988; Krishna and Murthy, 2004; Lazaridis et al., 2007; Lazaridis et al., 2011; Lee and Oh, 1999a; Lee and Oh, 1999b; Mobius and Santen, 1996; Riley, 1992; Simoes, 1990; van

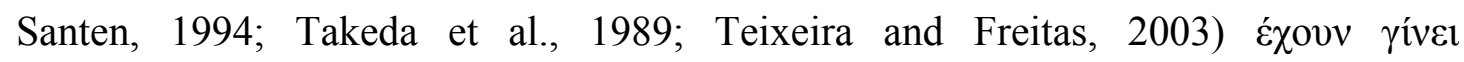

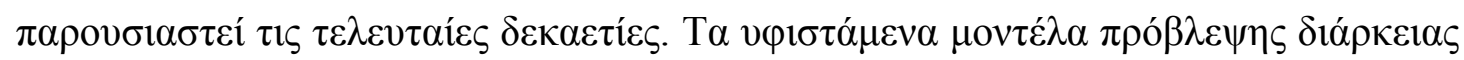

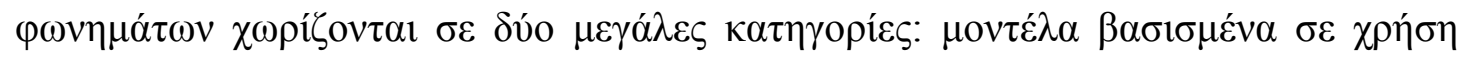

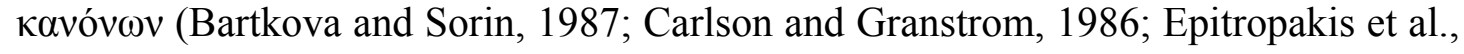




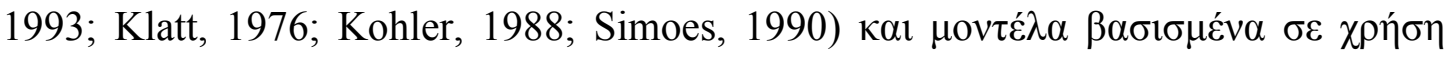
$\delta \varepsilon \delta o \mu \varepsilon ́ v \omega v$ (Chung, 2002; Goubanova and King, 2008; Iwahashi and Sagisaka, 2000; Krishna and Murthy, 2004; Lazaridis et al., 2007; Lazaridis et al., 2011; Lee and Oh, 1999a; Lee and Oh, 1999b; Mobius and Santen, 1996; Riley, 1992; van Santen, 1994; Takeda et al., 1989; Teixeira and Freitas, 2003).

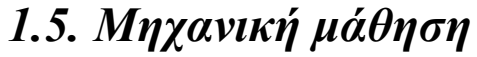

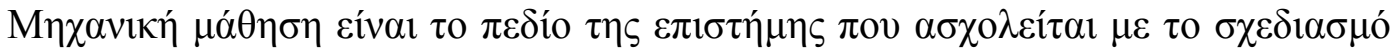

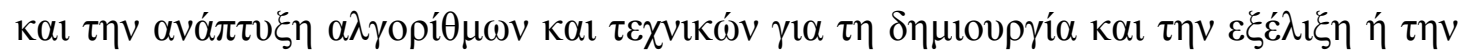

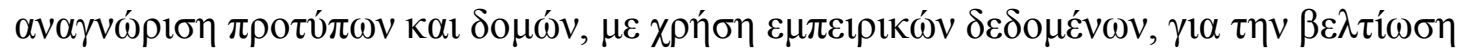

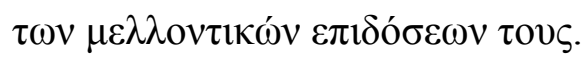

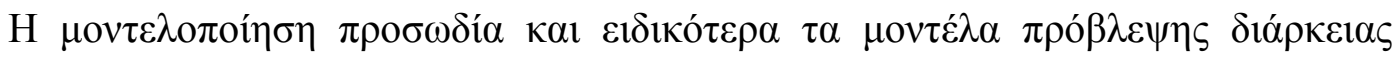
$\varphi \omega v \eta \mu \alpha ́ \tau \omega v \alpha \pi \circ \tau \varepsilon \lambda o v ́ v \pi \rho o ́ \beta \lambda \eta \mu \alpha \alpha \rho \imath \theta \mu \eta \tau \imath \kappa \eta ́ \varsigma ~ \pi \alpha \lambda \iota v \delta \rho o ́ \mu \eta \sigma \eta \varsigma$ (regression problem).

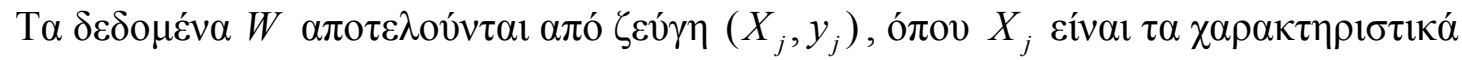

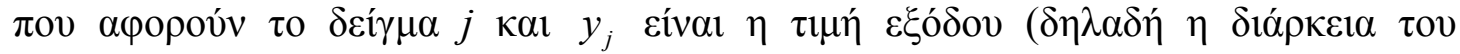

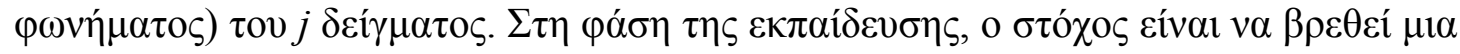

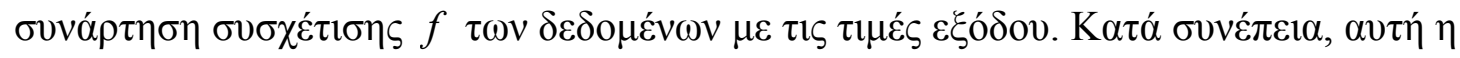

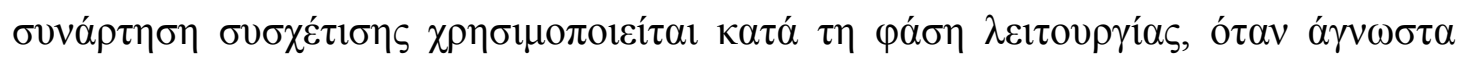

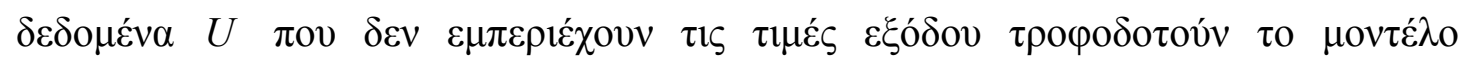

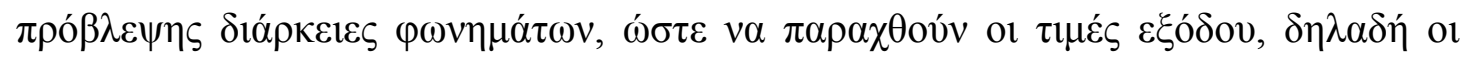

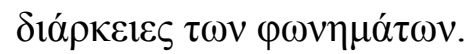


Training phase

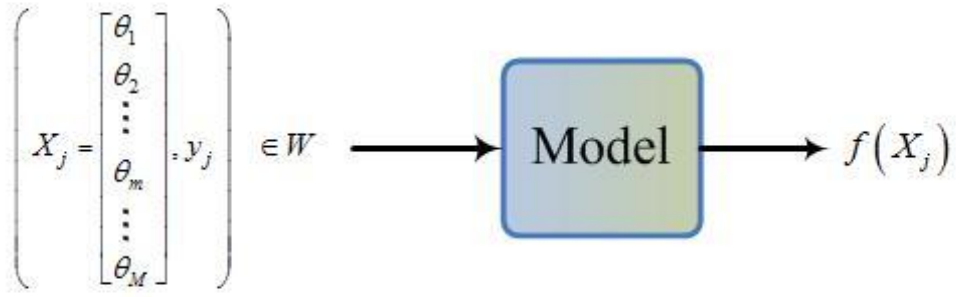

Operational phase

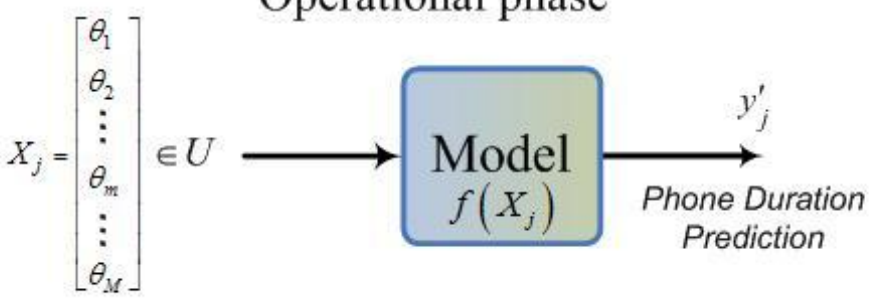

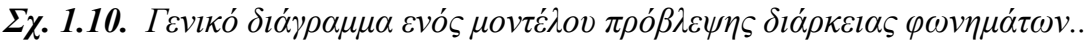

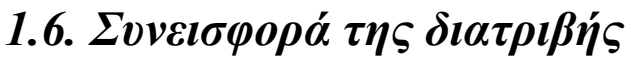

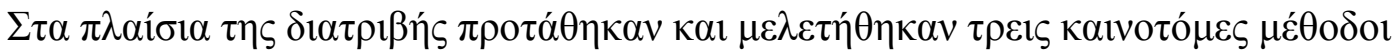

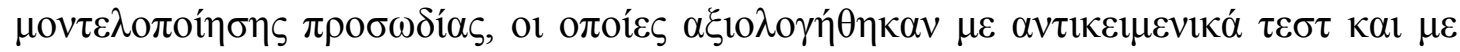

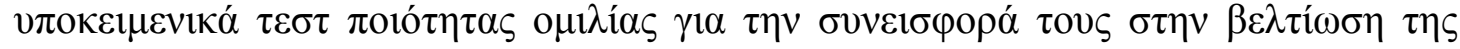

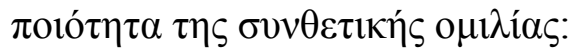

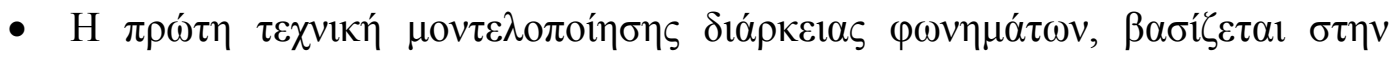

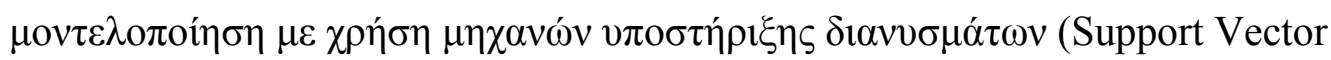

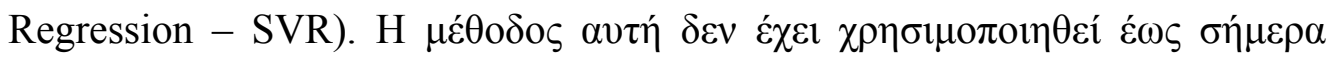

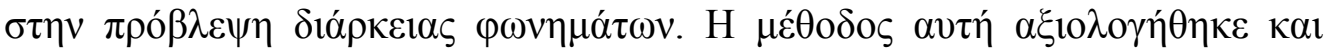

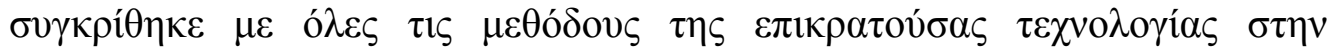

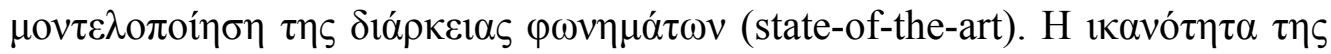

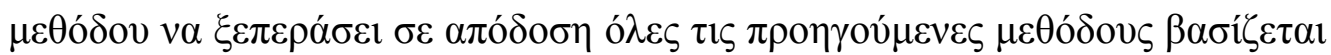

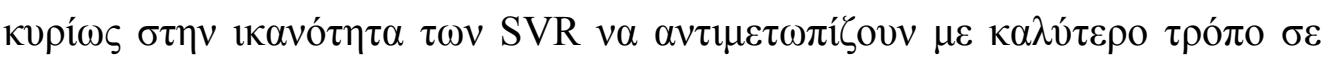

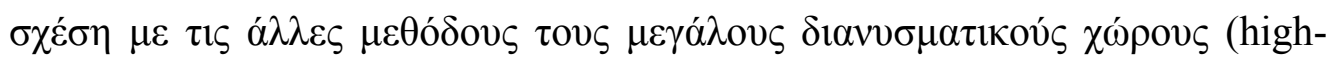

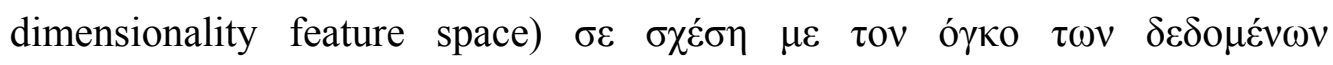

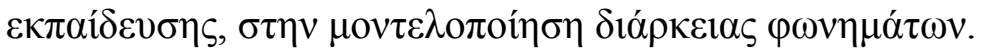




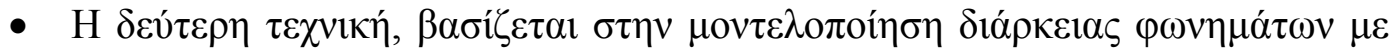

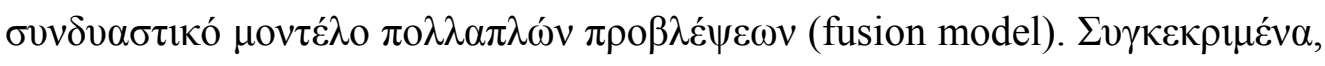

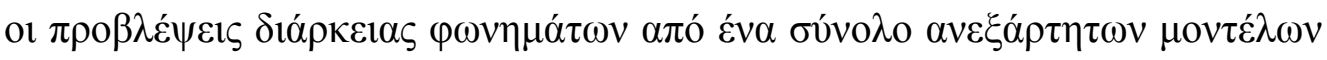

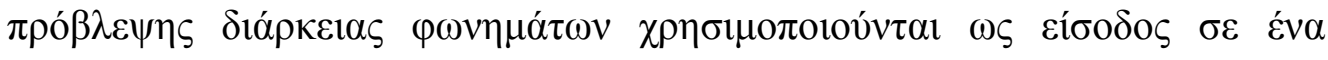

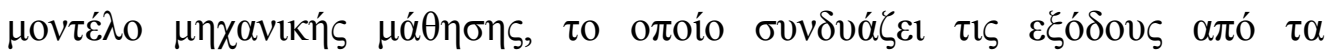

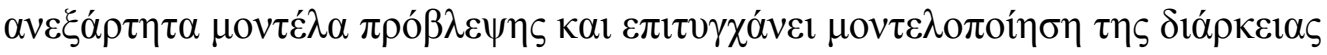

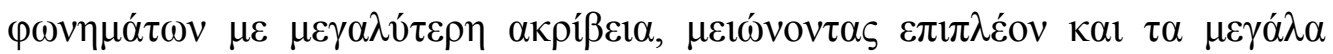

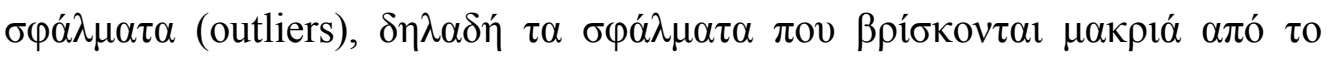

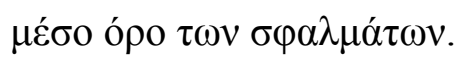

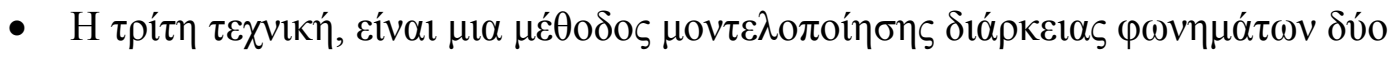

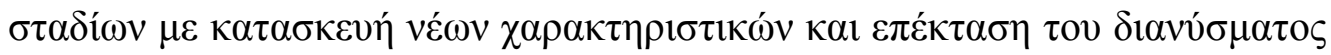

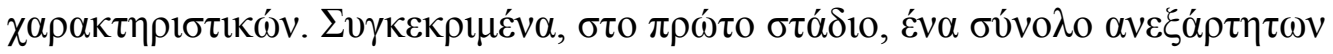

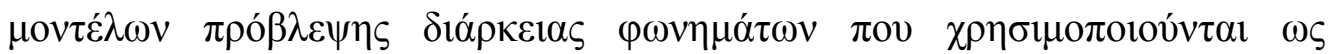

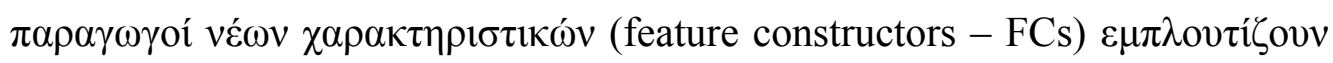

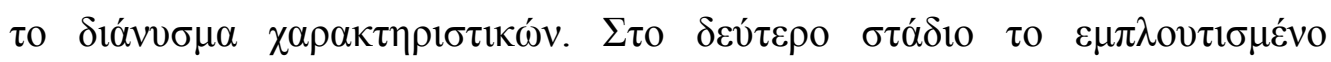

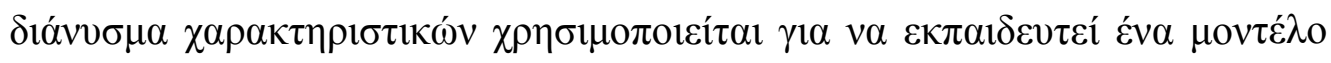

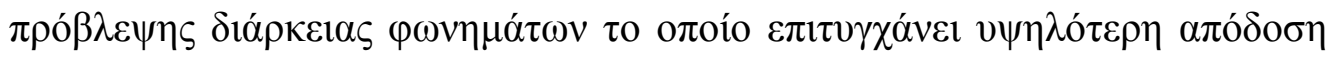

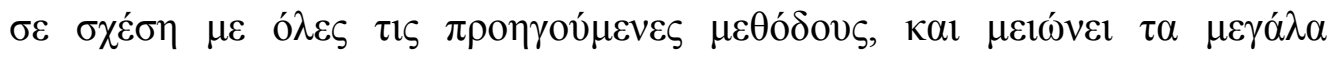

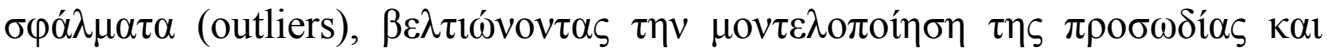

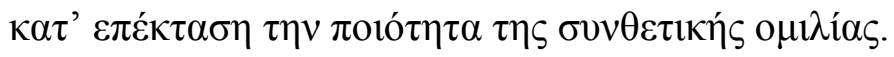

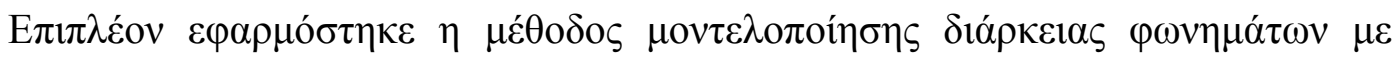

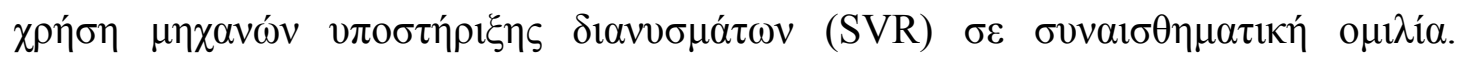

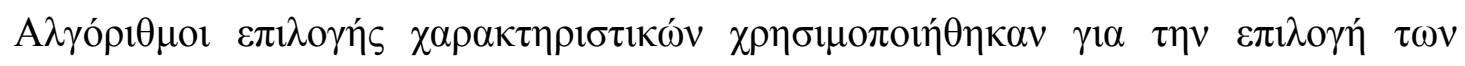

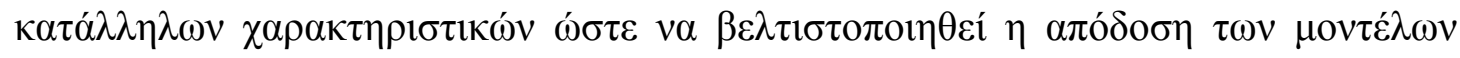

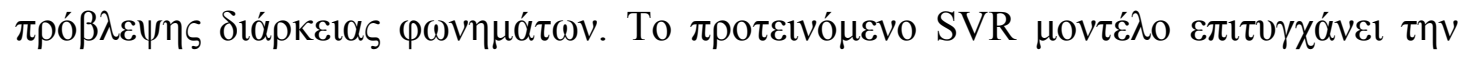

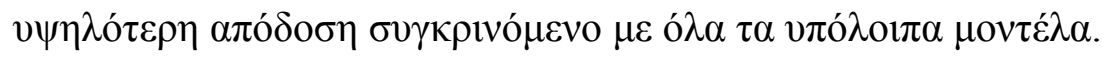

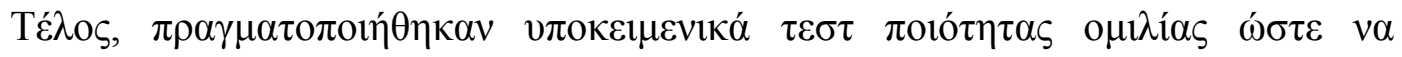

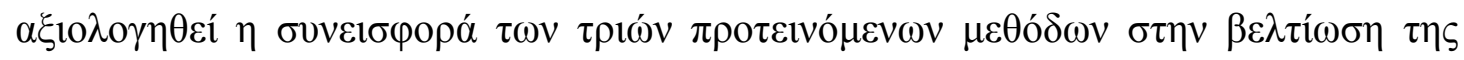

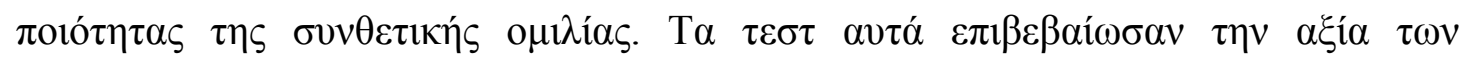

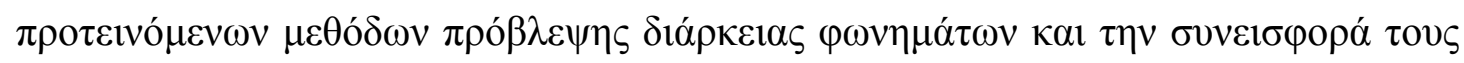

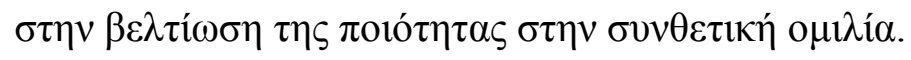




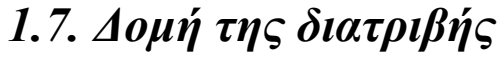

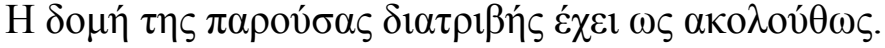

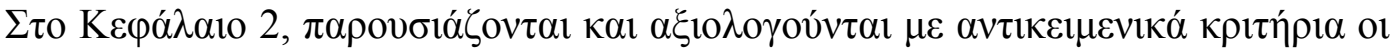

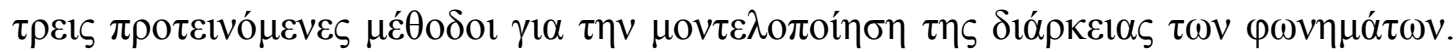

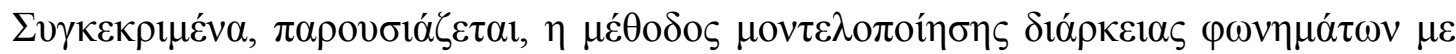

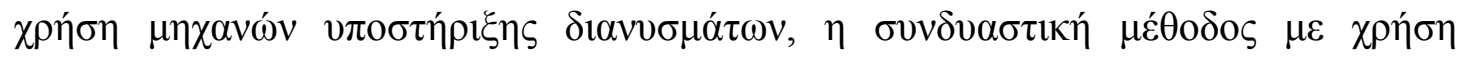

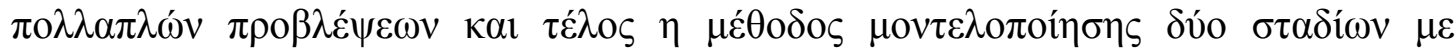

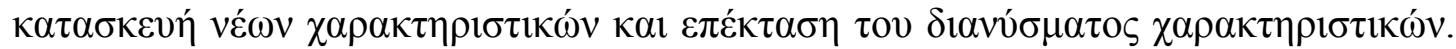

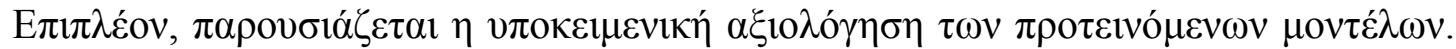

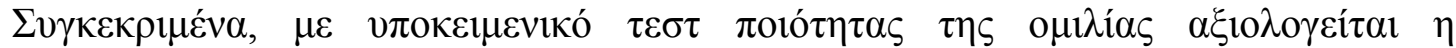

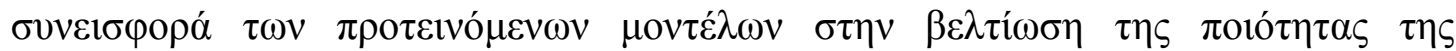

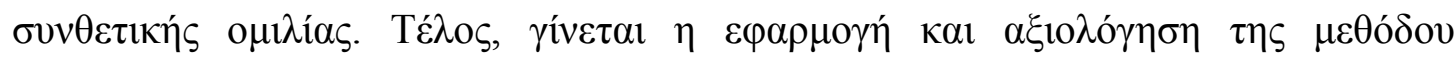

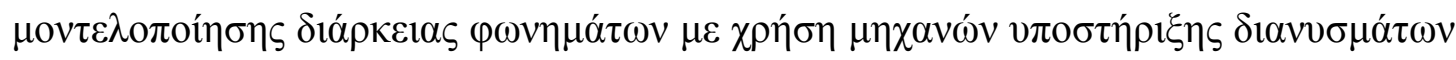

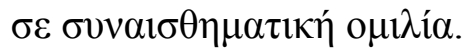

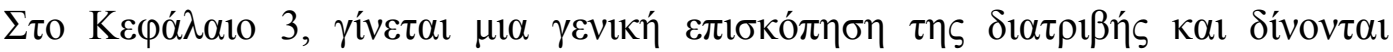

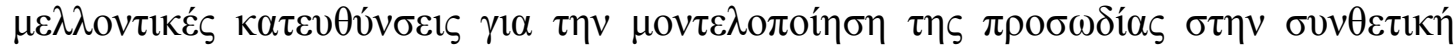

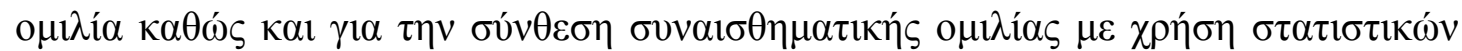

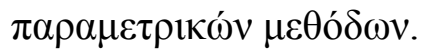




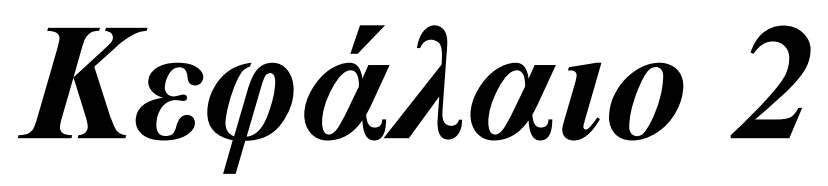

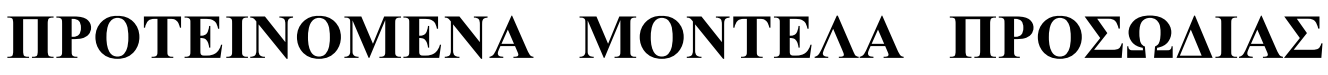

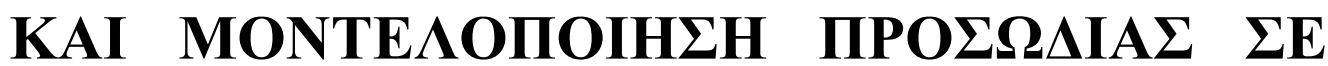

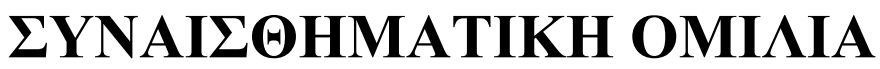

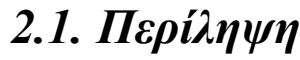

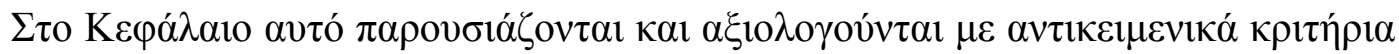

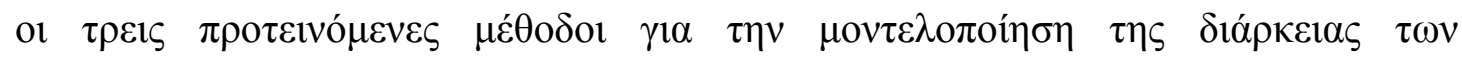

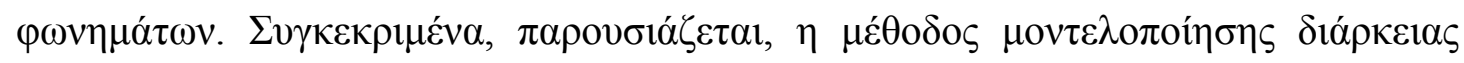

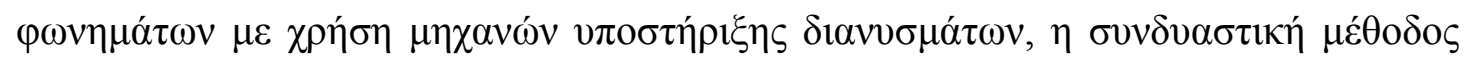

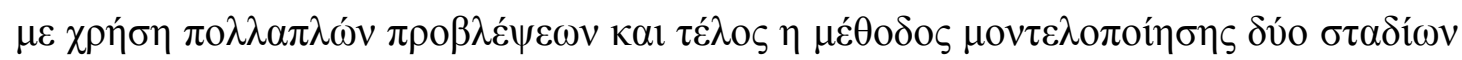

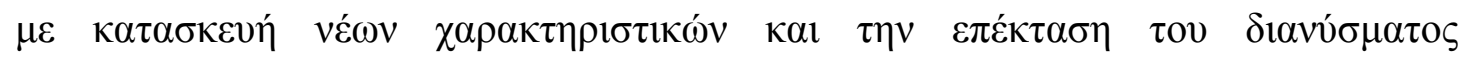

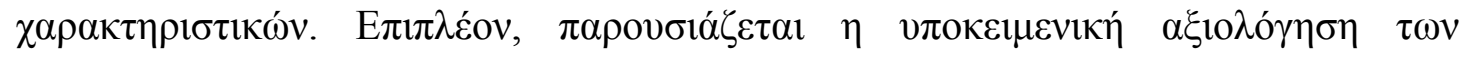

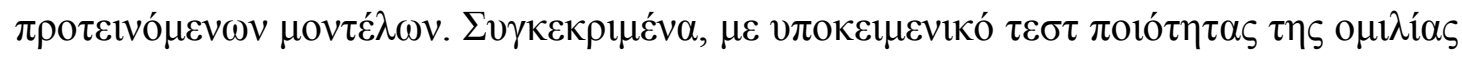

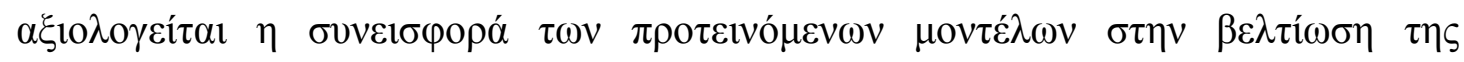

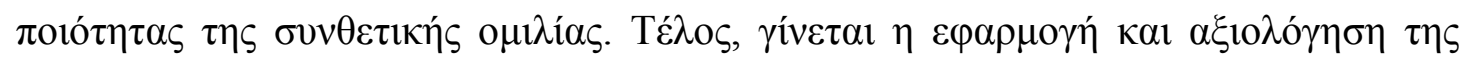

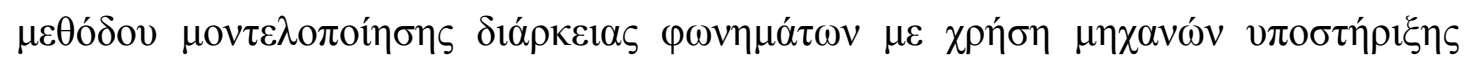

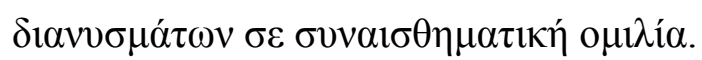




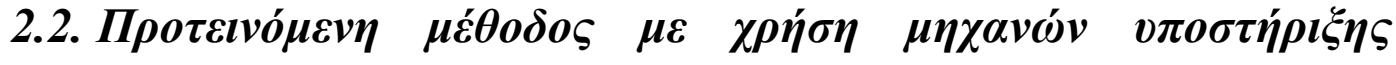 $\delta \imath \alpha v v \sigma \mu \alpha ́ \tau \omega v$}

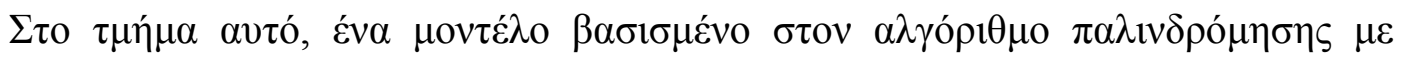

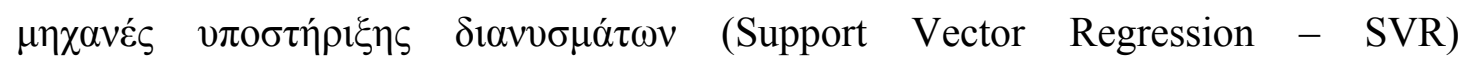

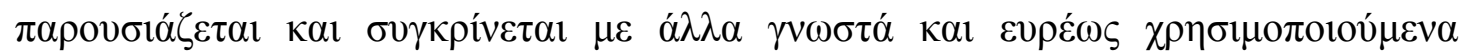

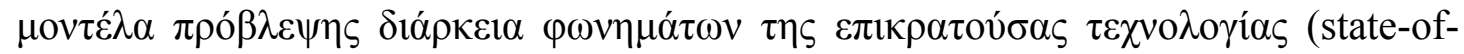

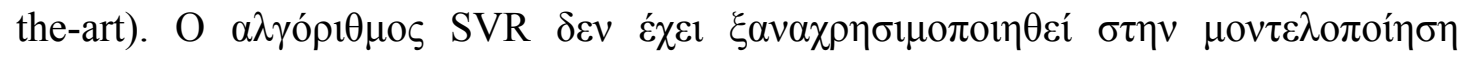

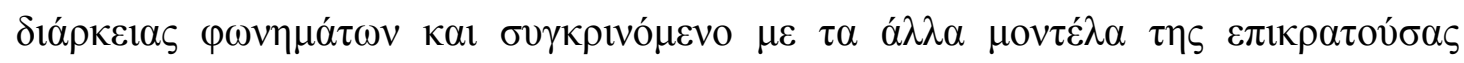

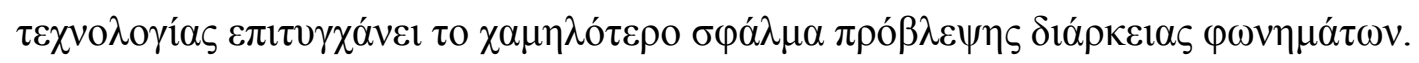

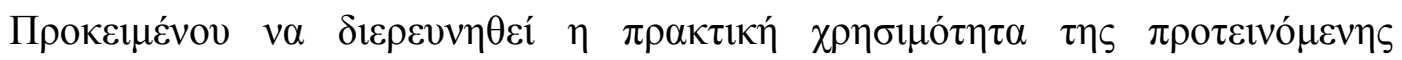

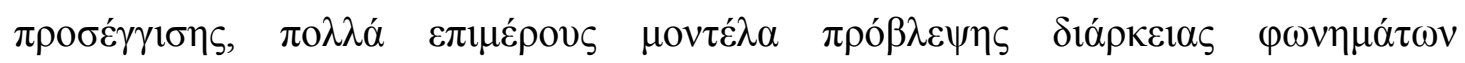

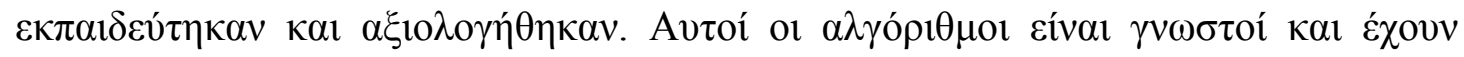

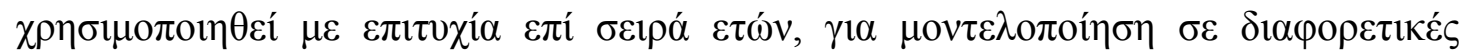

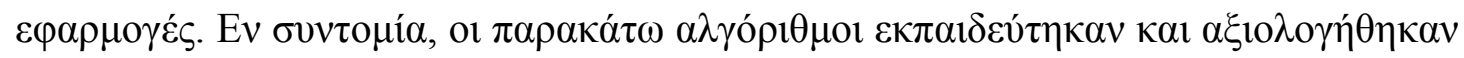

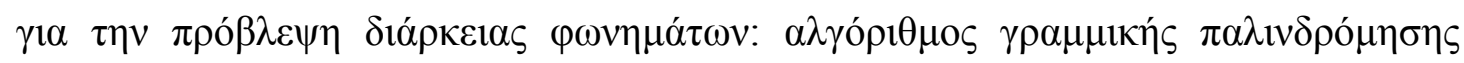
(linear regression), $\delta \varepsilon ́ v \tau \rho \alpha \alpha \pi$ ó $\alpha \sigma\rceil \varsigma$ (decision trees - regression and model trees),

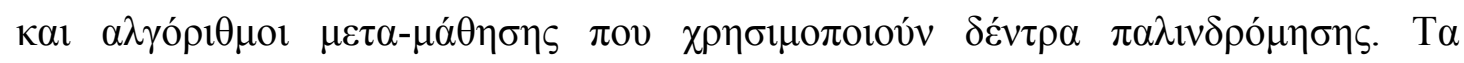

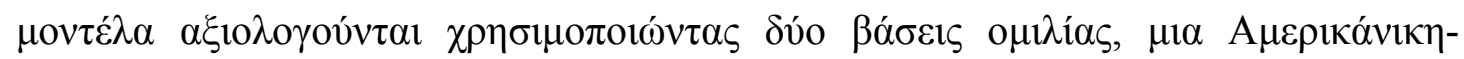

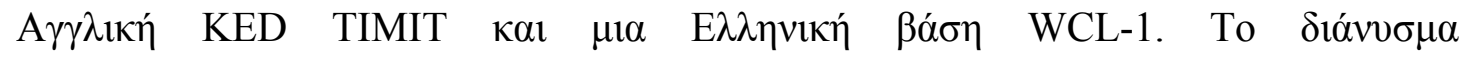

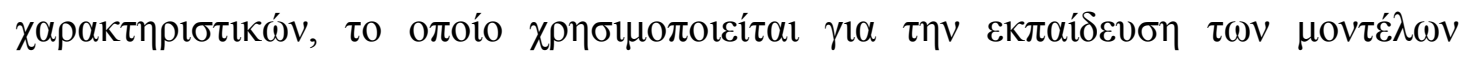

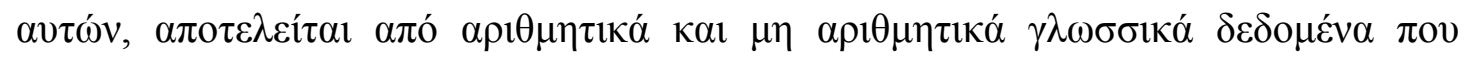

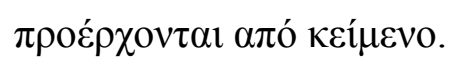

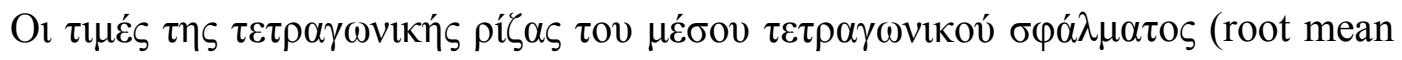

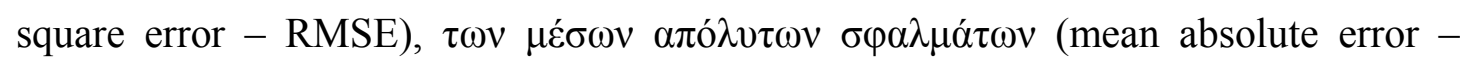

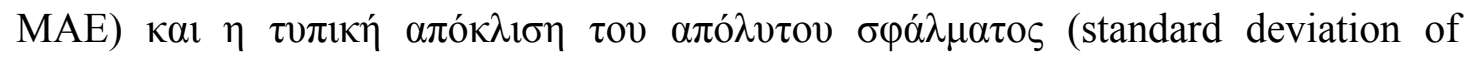

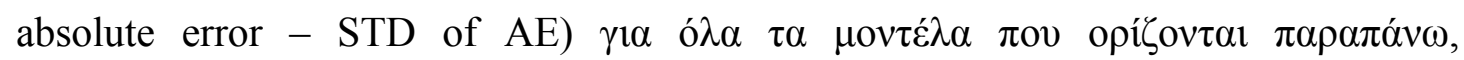

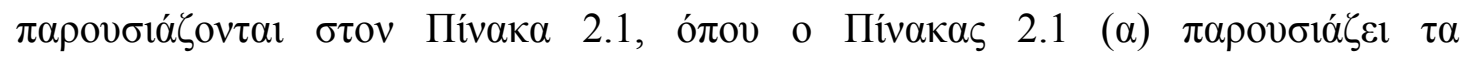

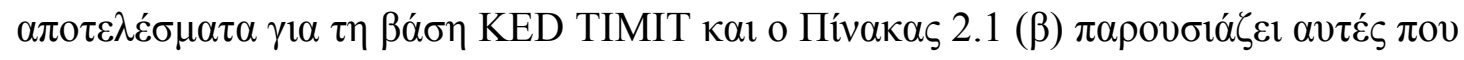

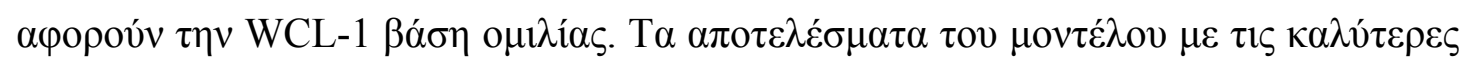

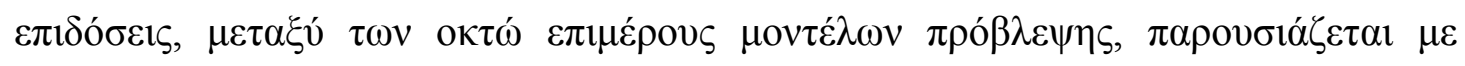

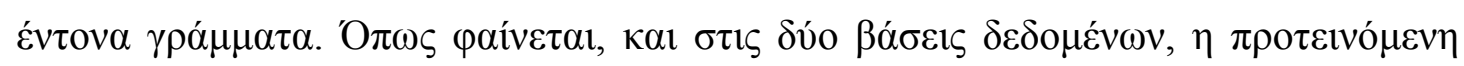




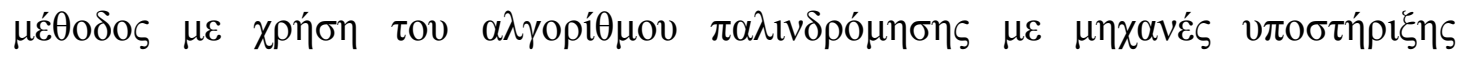

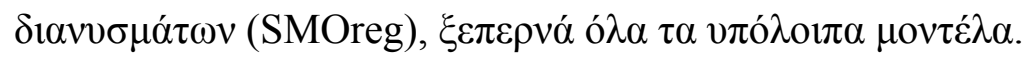

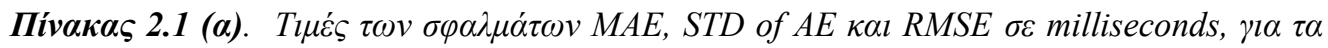

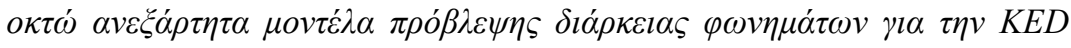

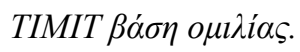

\begin{tabular}{lccc}
\hline Individual models (KED TIMIT database) & MAE $(\boldsymbol{m s})$ & $\boldsymbol{S T D}$ of $\boldsymbol{A E}(\boldsymbol{m s})$ & $\boldsymbol{R} M \boldsymbol{S} \boldsymbol{E}(\boldsymbol{m s})$ \\
\hline SMOreg & $\mathbf{1 4 . 9 5}$ & $\mathbf{1 4 . 1 1}$ & $\mathbf{2 0 . 5 6}$ \\
Add. Reg. m5pR & 15.82 & 14.34 & 21.35 \\
Add. Reg. REPTrees & 16.29 & 15.06 & 22.19 \\
Bagging m5pR & 16.51 & 14.76 & 22.14 \\
m5p & 16.62 & 14.77 & 22.23 \\
Bagging REPTrees & 16.69 & 15.89 & 23.04 \\
m5pR & 16.93 & 15.16 & 22.72 \\
Linear Regression & 17.15 & 15.16 & 22.89 \\
\hline
\end{tabular}

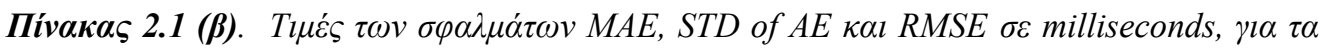

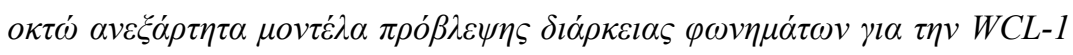

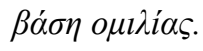

\begin{tabular}{lccc}
\hline Individual models (WCL-1 database) & MAE $(\boldsymbol{m s})$ & STD of $\boldsymbol{A E}(\boldsymbol{m s})$ & $\boldsymbol{R M S E}(\boldsymbol{m s})$ \\
\hline SMOreg & $\mathbf{1 6 . 7 8}$ & $\mathbf{1 8 . 8 1}$ & $\mathbf{2 5 . 2 1}$ \\
Linear Regression & 18.00 & 19.02 & 26.19 \\
Add. Reg. REPTrees & 18.08 & 19.97 & 26.94 \\
Add. Reg. m5pR & 18.13 & 19.16 & 26.38 \\
Bagging m5pR & 18.14 & 19.63 & 26.72 \\
m5p & 18.31 & 20.08 & 27.17 \\
Bagging REPTrees & 18.93 & 20.32 & 27.77 \\
m5pR & 19.07 & 20.10 & 27.71 \\
\hline
\end{tabular}

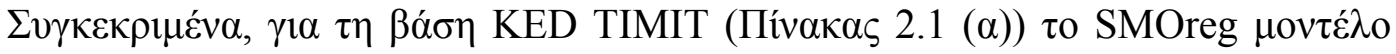

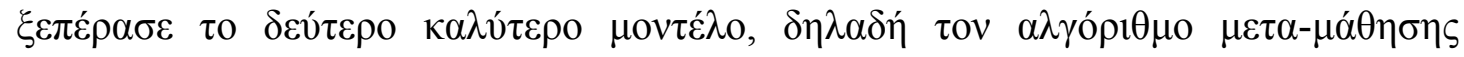

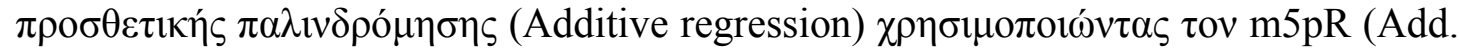

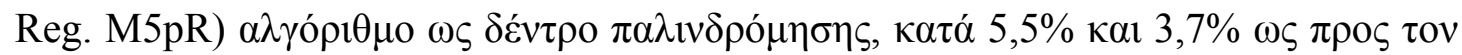

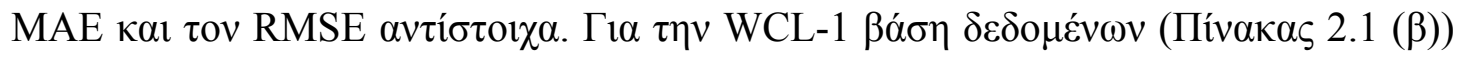

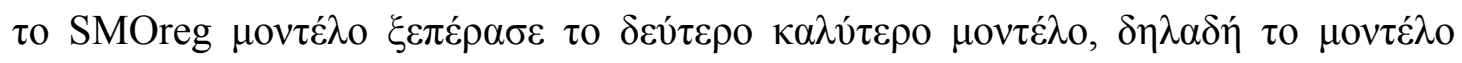

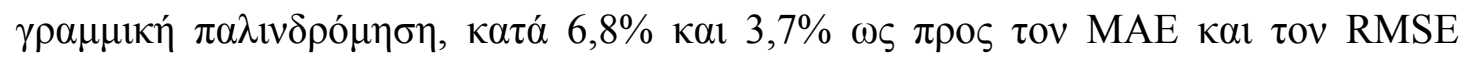

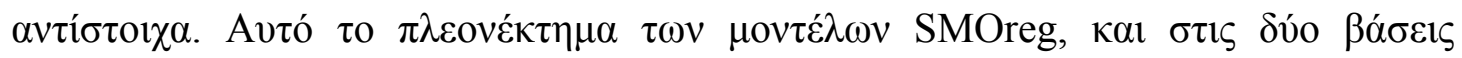

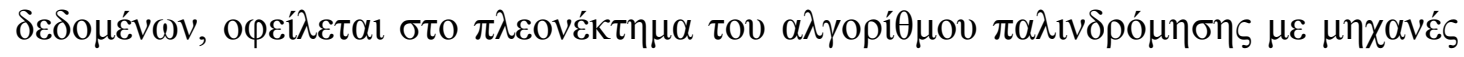

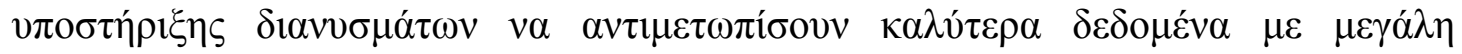




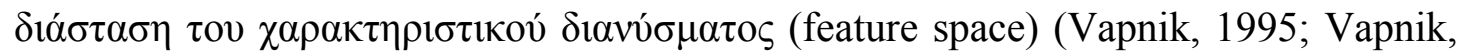

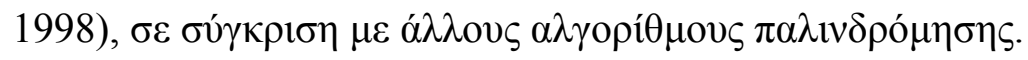

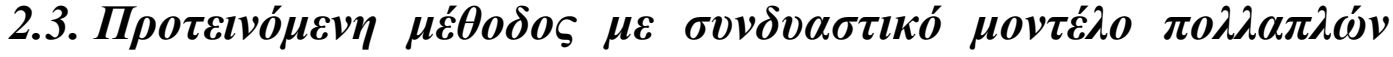 $\pi \rho \sigma \beta \lambda \varepsilon ́ \psi \varepsilon \omega v$}

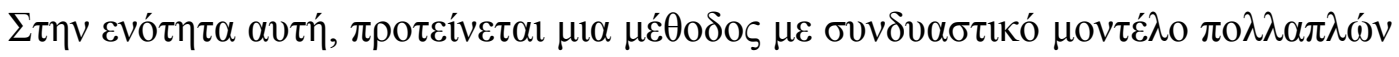

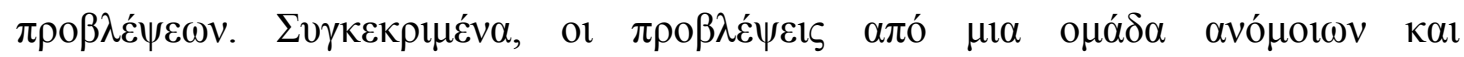

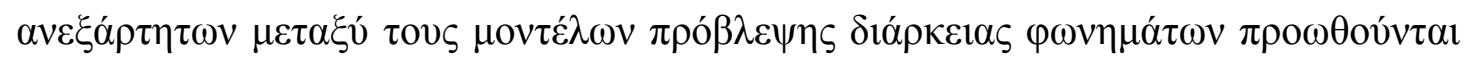

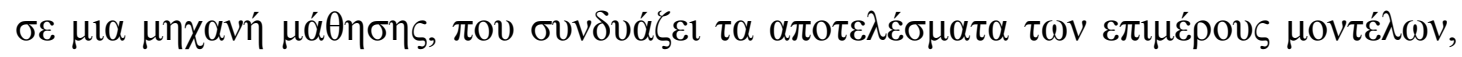

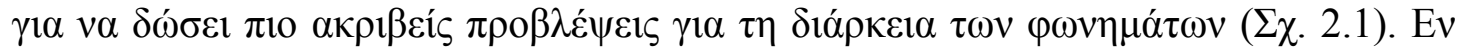

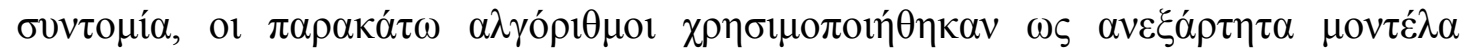

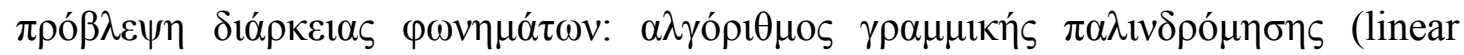

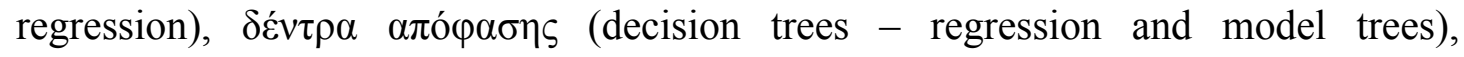

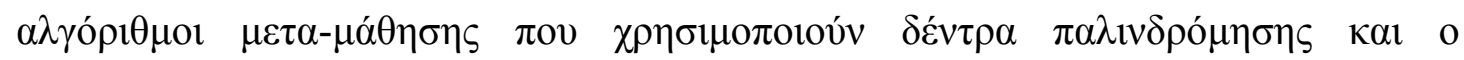

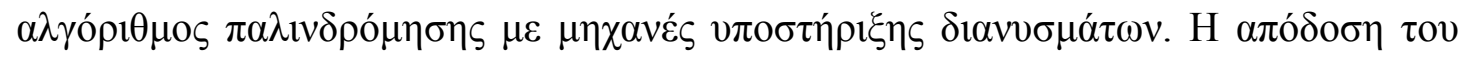

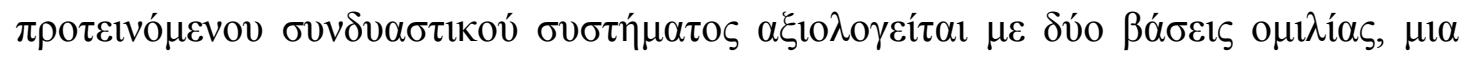

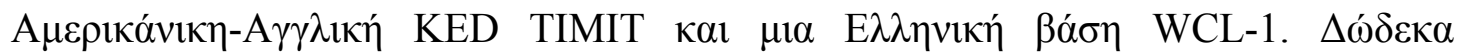

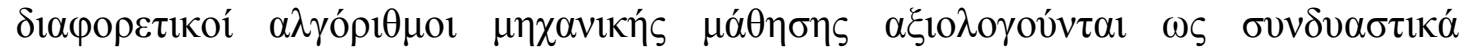

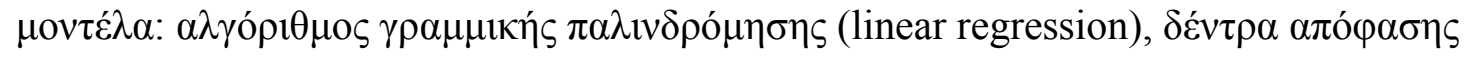

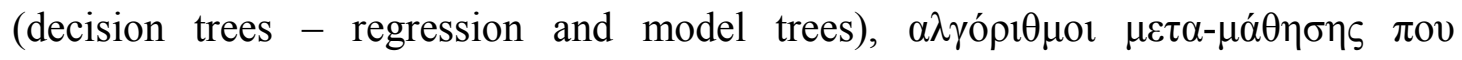

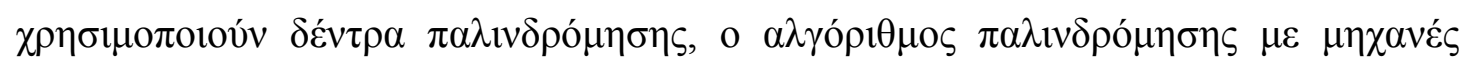

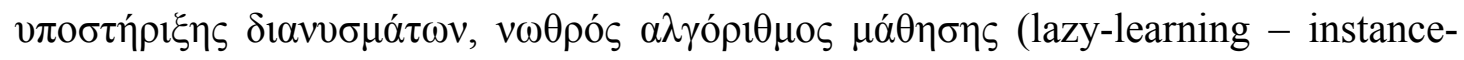

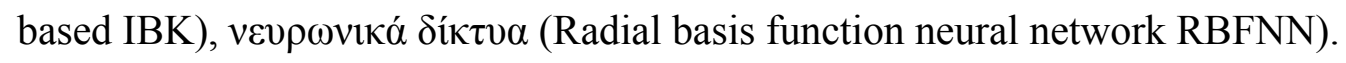




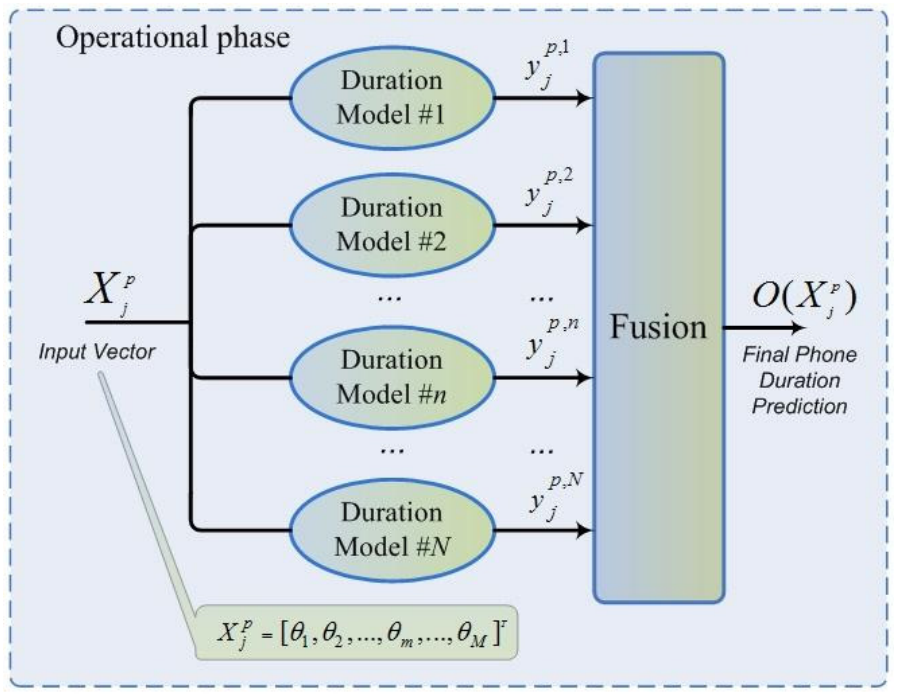

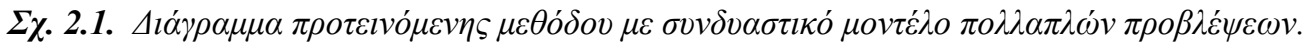

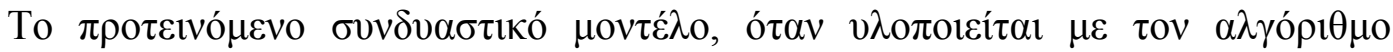

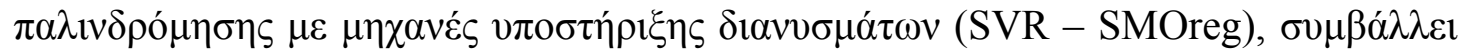

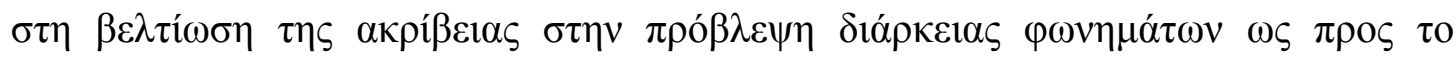

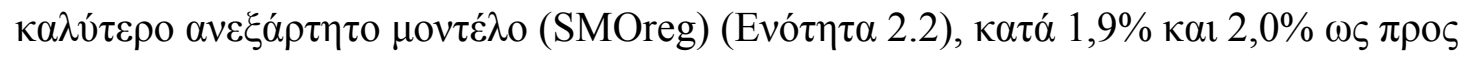

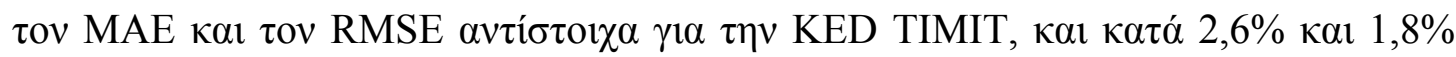

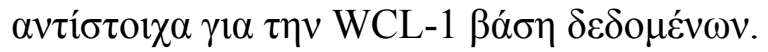

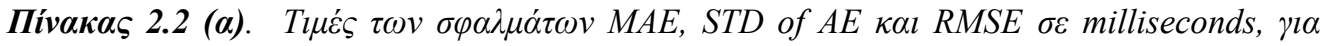

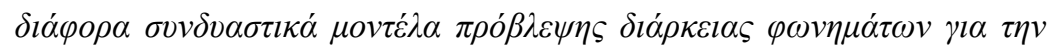

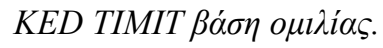

\begin{tabular}{lccc}
\hline KED TIMIT database & MAE $(\mathbf{m s})$ & STD of $\boldsymbol{A E}(\boldsymbol{m s})$ & $\boldsymbol{R M S E}(\mathbf{m s})$ \\
\hline SMOreg & $\mathbf{1 4 . 6 6}$ & $\mathbf{1 3 . 8 2}$ & $\mathbf{2 0 . 1 4}$ \\
IBK & 15.19 & 14.69 & 21.02 \\
Linear Regression & 15.49 & 14.45 & 21.18 \\
RBFNN & 15.53 & 14.49 & 21.24 \\
m5p & 15.56 & 14.60 & 21.34 \\
Add. Regr. m5pR & 15.72 & 14.94 & 21.69 \\
Add. Regr. REPTrees & 15.79 & 14.94 & 21.74 \\
Bagging m5pR & 15.81 & 15.09 & 21.86 \\
Bagging REPTrees & 15.88 & 15.15 & 21.95 \\
m5pR & 15.97 & 15.28 & 22.10 \\
\hline No fusion - best individual model, & 14.95 & 14.11 & 20.56 \\
SMOreg & &
\end{tabular}




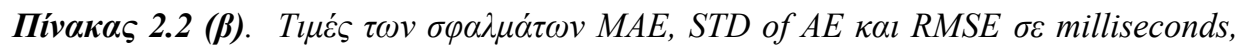

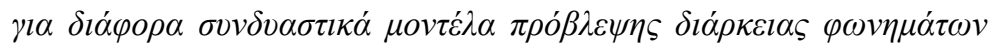

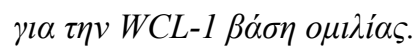

\begin{tabular}{lccc}
\hline WCL-1 database & MAE $(\boldsymbol{m s})$ & STD of AE $(\boldsymbol{m s})$ & $\boldsymbol{R M S E}(\boldsymbol{m s})$ \\
\hline SMOreg & $\mathbf{1 6 . 3 5}$ & $\mathbf{1 8 . 5 9}$ & $\mathbf{2 4 . 7 6}$ \\
IBK & 16.98 & 18.85 & 25.47 \\
RBFNN & 17.34 & 19.51 & 26.10 \\
Add. Regr. m5pR & 17.69 & 19.84 & 26.58 \\
Bagging m5pR & 17.72 & 19.84 & 26.60 \\
m5p & 17.84 & 20.51 & 27.18 \\
m5pR & 17.91 & 20.00 & 26.85 \\
Bagging REPTrees & 17.99 & 20.45 & 27.23 \\
Add. Regr. REPTrees & 18.00 & 20.56 & 27.32 \\
Linear Regression & 18.32 & 20.19 & 27.26 \\
\hline No fusion - best individual model, & 16.78 & 18.81 & 25.21 \\
SMOreg & & &
\end{tabular}

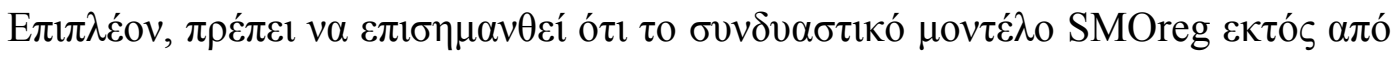
$\tau \eta \mu \varepsilon i ́ \omega \sigma \eta ~ \tau o v ~ \sigma v v o \lambda \iota \kappa o v ́ ~ \sigma \varphi \alpha ́ \lambda \mu \alpha \tau o \varsigma ~ \mu \varepsilon i ́ \omega \sigma \varepsilon ~ \varepsilon \pi i ́ \sigma \eta \varsigma ~ \tau \alpha ~ \alpha \kappa \rho \alpha i ́ \alpha ~ \sigma \varphi \alpha ́ \lambda \mu \alpha \tau \alpha$ (outliers).

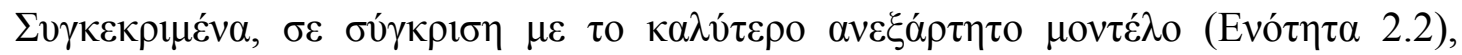

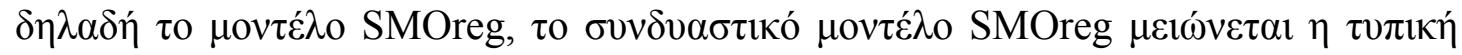

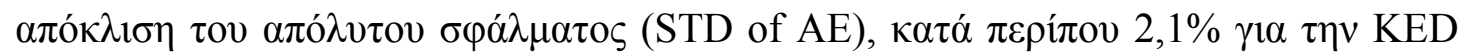

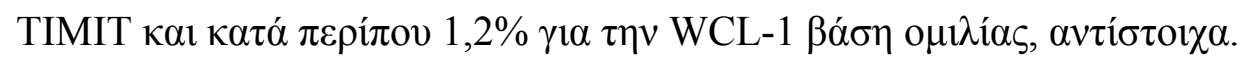

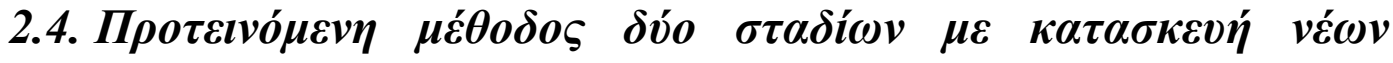

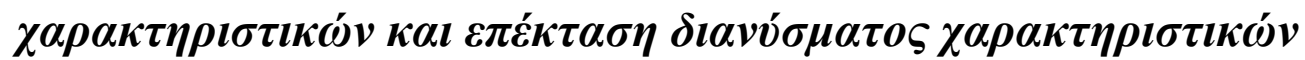

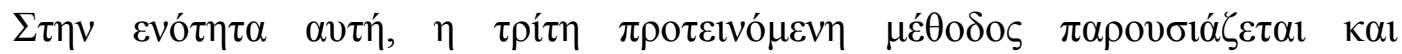

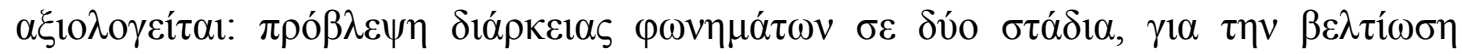

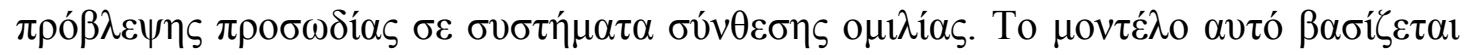

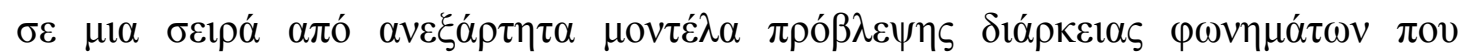

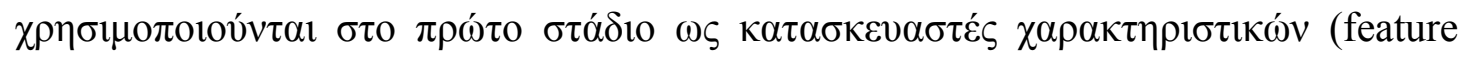

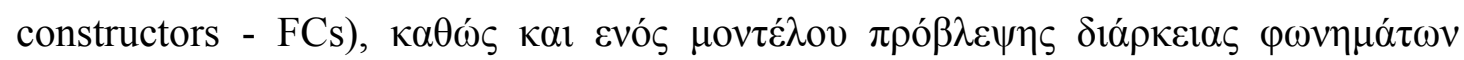

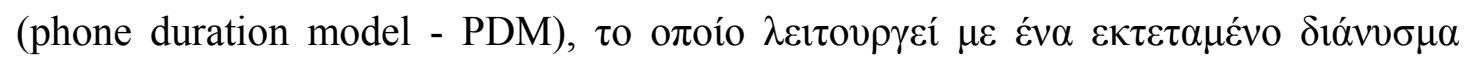

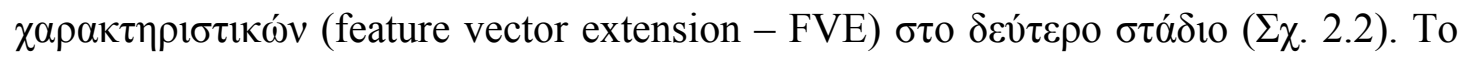

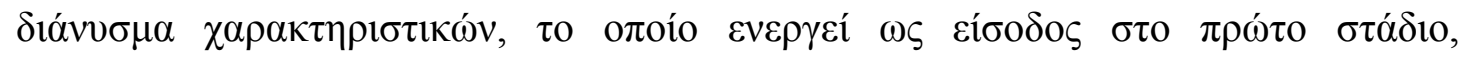

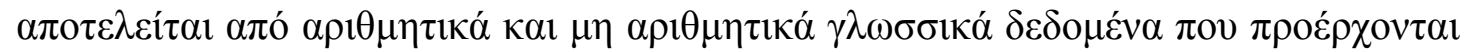




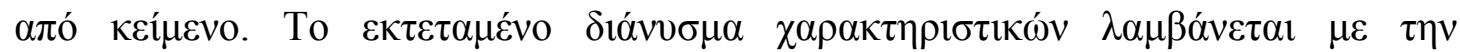

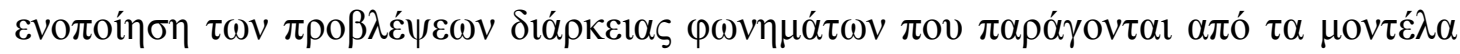

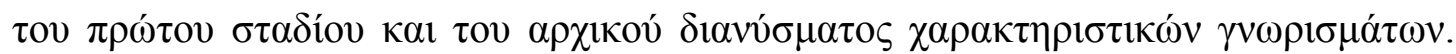

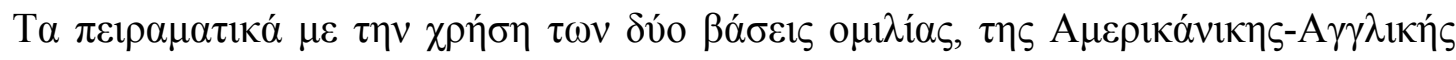

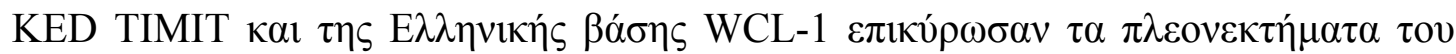

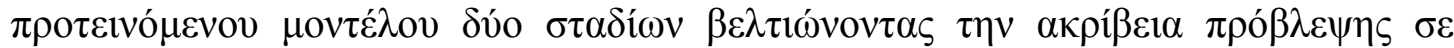

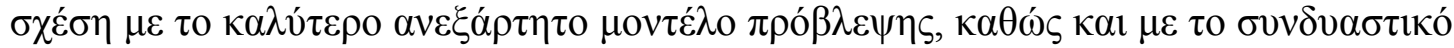

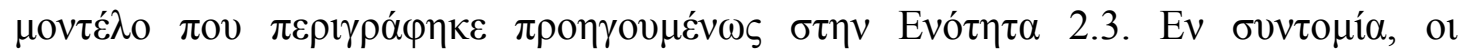

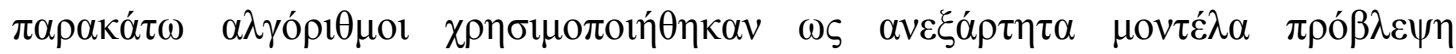

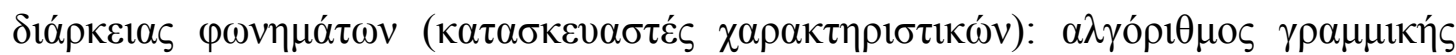

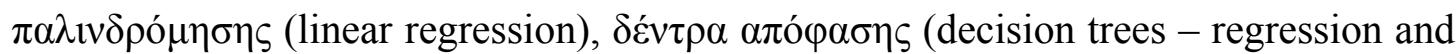

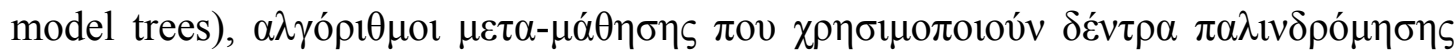

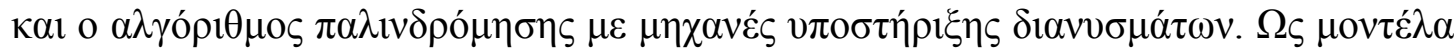

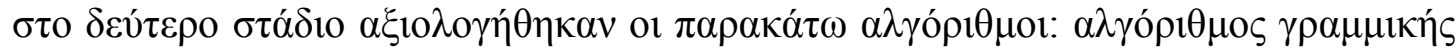

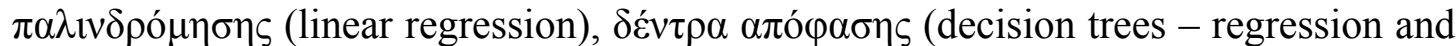

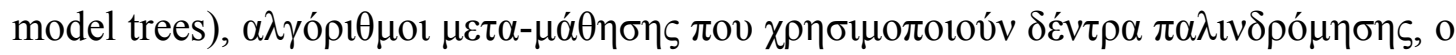

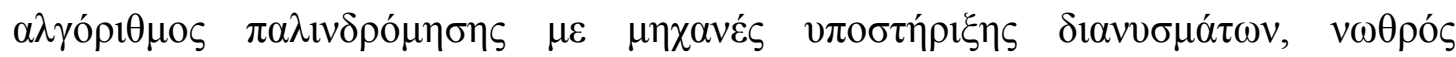

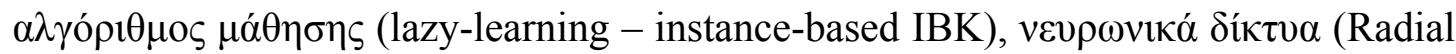
basis function neural network RBFNN).

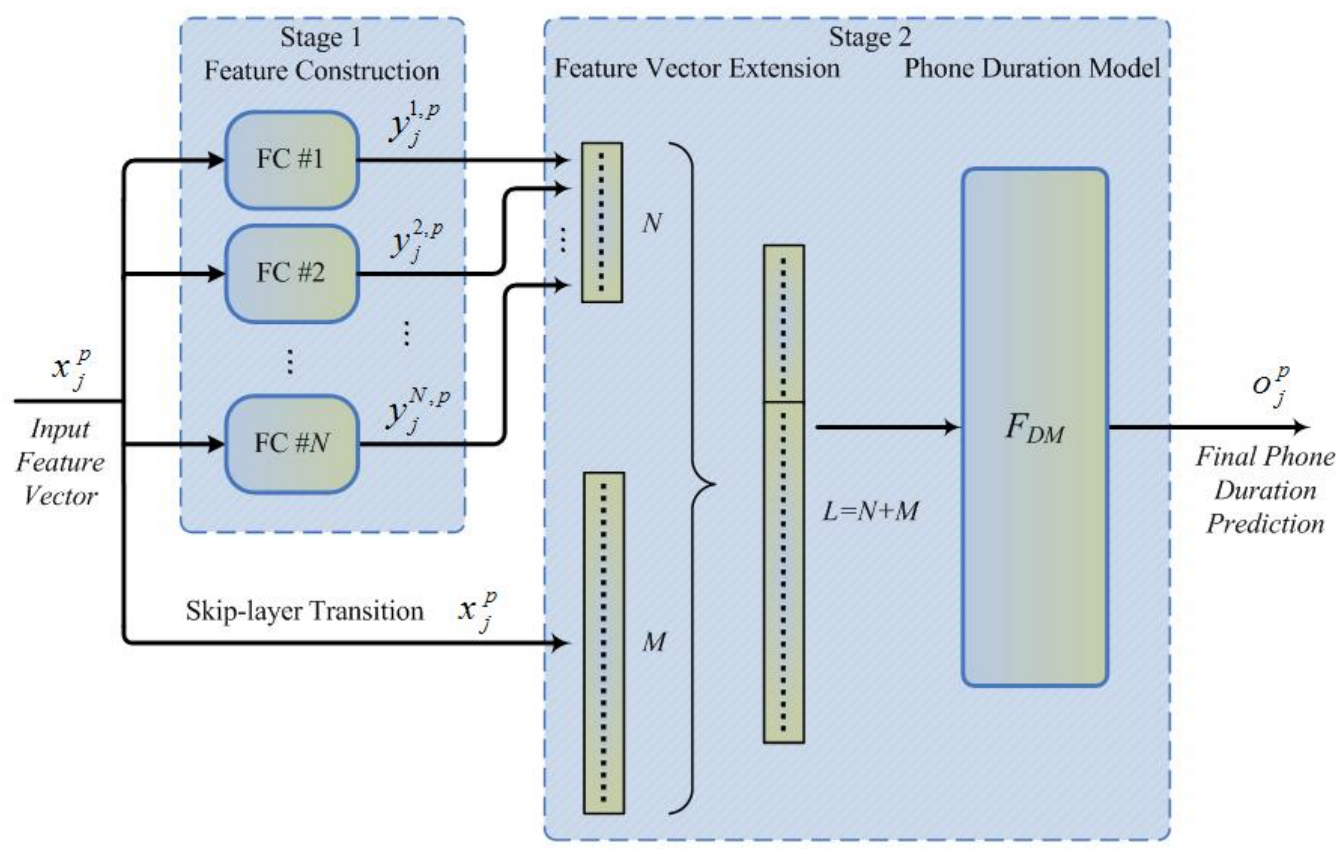

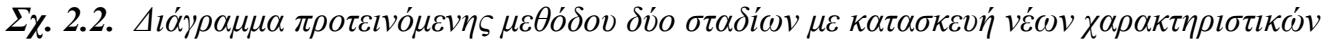

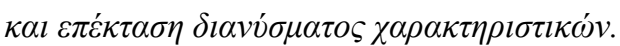




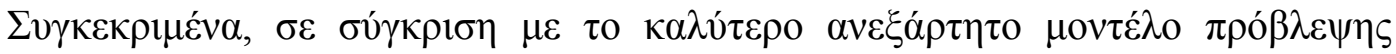

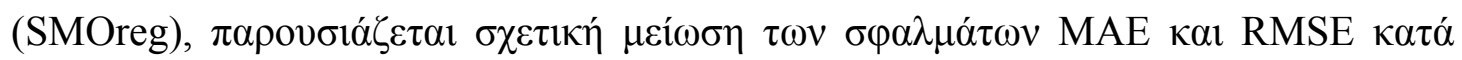

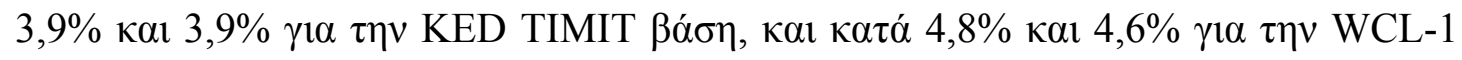

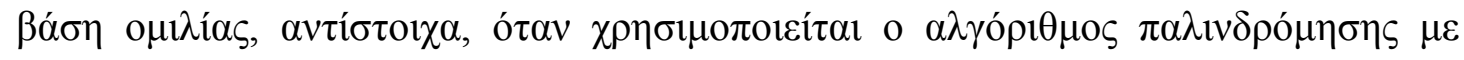

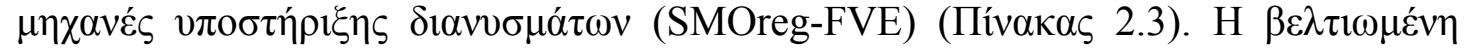

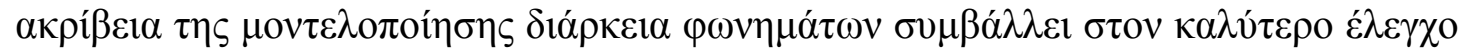

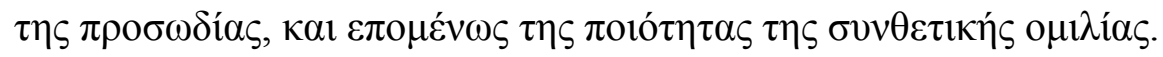

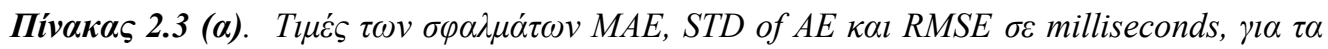

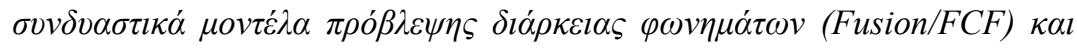

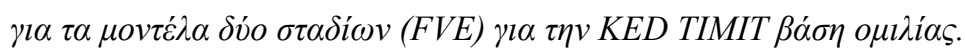

\begin{tabular}{lcccccc}
\hline \multirow{2}{*}{ PDM algorithms } & \multicolumn{3}{c}{ baseline, FCF } & \multicolumn{4}{c}{ proposed scheme, FVE } \\
\cline { 2 - 7 } & MAE & STD of AE & RMSE & MAE & STD of AE RMSE \\
\hline Add. Regr. m5pR & 15.72 & 14.94 & 21.69 & 15.74 & 14.92 & 21.69 \\
Add. Regr. REPTrees & 15.79 & 14.94 & 21.74 & 15.60 & 14.76 & 21.47 \\
Bagg. m5pR & 15.81 & 15.09 & 21.86 & 15.83 & 15.08 & 21.86 \\
Bagg. REPTrees & 15.88 & 15.15 & 21.95 & 16.26 & 15.52 & 22.48 \\
IBK & 15.19 & 14.69 & 21.02 & 17.41 & 15.88 & 23.57 \\
LR & 15.49 & 14.45 & 21.18 & 15.40 & 14.40 & 21.08 \\
m5p & 15.56 & 14.60 & 21.34 & 15.45 & 14.48 & 21.17 \\
m5pR & 15.97 & 15.28 & 22.10 & 15.95 & 15.25 & 22.06 \\
RBFNN & 15.53 & 14.49 & 21.24 & 21.28 & 18.37 & 28.11 \\
SMOreg & 14.66 & 13.82 & 20.14 & $\mathbf{1 4 . 3 6}$ & $\mathbf{1 3 . 5 6}$ & $\mathbf{1 9 . 7 5}$ \\
\hline best FC (SMOreg) & - & - & - & 14.95 & 14.11 & 20.56 \\
\hline
\end{tabular}

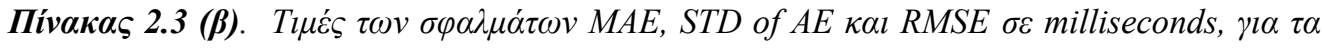

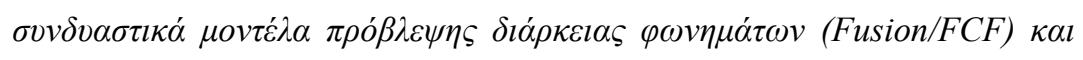

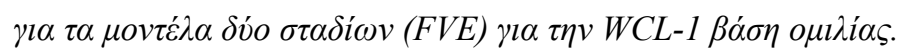

\begin{tabular}{lcccccc}
\hline \multirow{2}{*}{ PDM algorithms } & \multicolumn{3}{c}{ baseline, FCF } & \multicolumn{4}{c}{ proposed scheme, FVE } \\
\cline { 2 - 7 } & MAE & STD of $\boldsymbol{A E}$ & $\boldsymbol{R} M S \boldsymbol{M}$ M & ME & STD of $\boldsymbol{A E}$ RMSE \\
\hline Add. Regr. m5pR & 17.69 & 19.84 & 26.58 & 17.75 & 19.93 & 26.69 \\
Add. Regr. REPTrees & 18.00 & 20.56 & 27.32 & 17.89 & 20.22 & 26.99 \\
Bagg. m5pR & 17.72 & 19.84 & 26.60 & 17.75 & 19.82 & 26.60 \\
Bagg. REPTrees & 17.99 & 20.45 & 27.23 & 18.15 & 20.51 & 27.39 \\
IBK & 16.98 & 18.85 & 25.47 & 18.53 & 20.29 & 27.48 \\
LR & 18.32 & 20.19 & 27.26 & 18.22 & 20.11 & 27.14 \\
m5p & 17.84 & 20.51 & 27.18 & 17.81 & 20.46 & 27.12 \\
m5pR & 17.91 & 20.00 & 26.85 & 17.98 & 20.02 & 26.91 \\
RBFNN & 17.34 & 19.51 & 26.10 & 21.29 & 21.43 & 30.21 \\
SMOreg & 16.35 & 18.59 & 24.76 & $\mathbf{1 5 . 9 7}$ & $\mathbf{1 8 . 3 4}$ & $\mathbf{2 4 . 0 4}$ \\
\hline best FC (SMOreg) & - & - & - & 16.78 & 18.81 & 25.21 \\
\hline
\end{tabular}




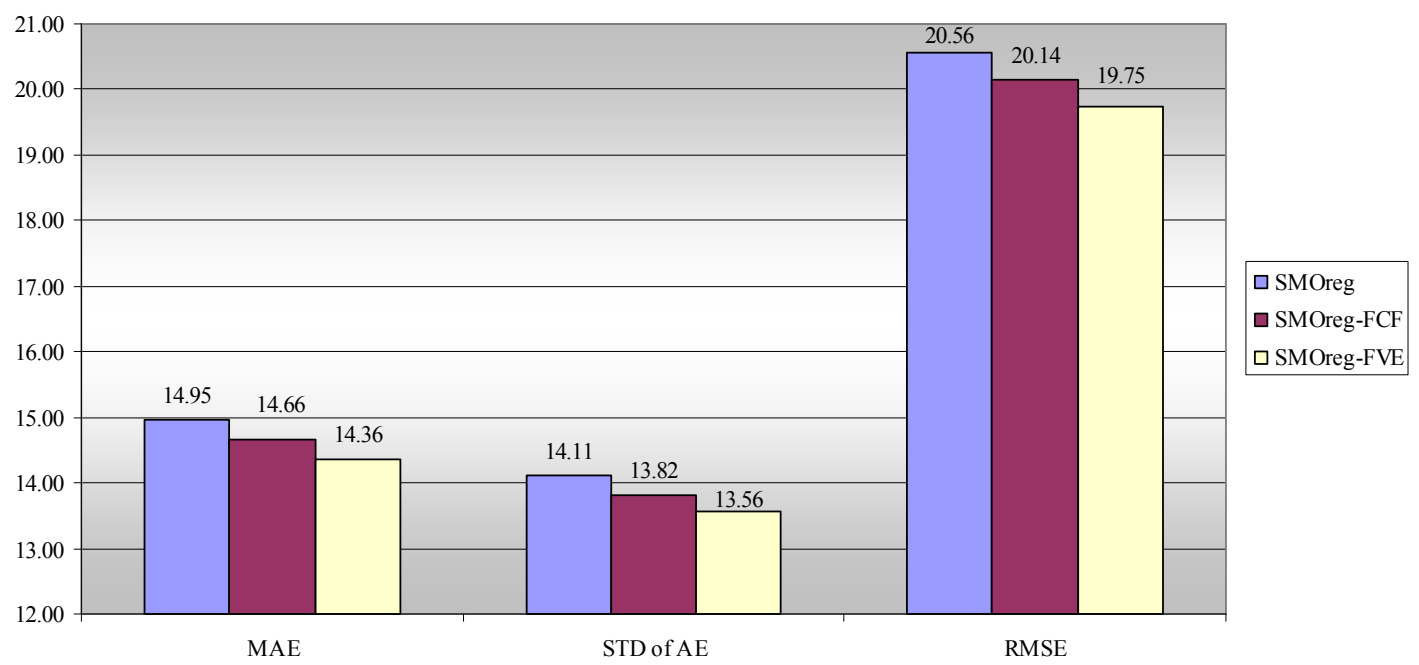

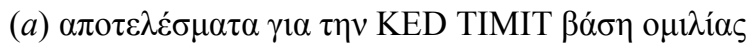

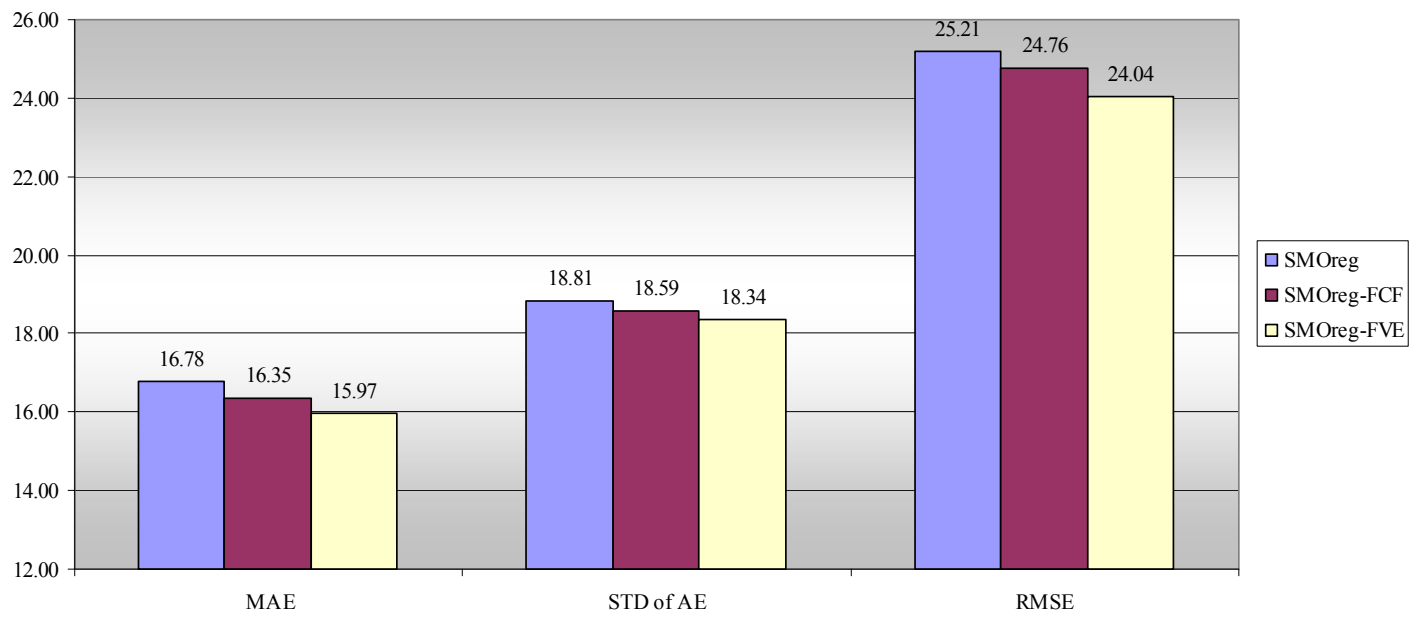

(b) $\alpha \pi 0 \tau \varepsilon \lambda \varepsilon \varepsilon \sigma \mu \alpha \tau \alpha \gamma 1 \alpha \tau \eta \nu$ WCL-1 $\beta \alpha ́ \sigma \eta ~ o \mu \imath \lambda i ́ \alpha \varsigma$

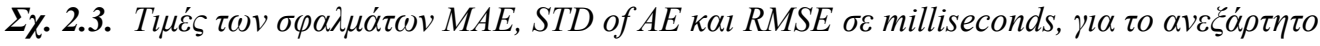

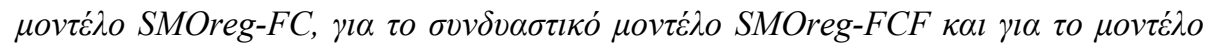

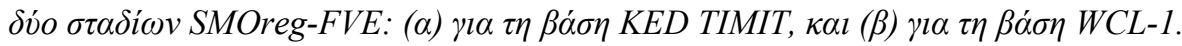

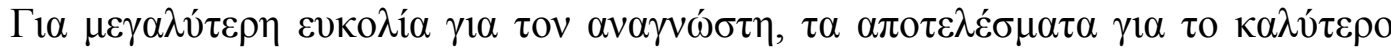

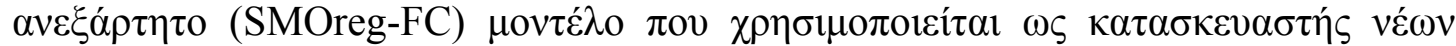

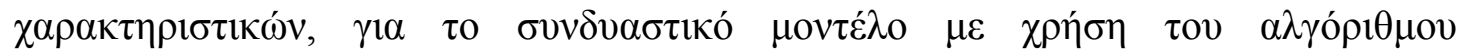

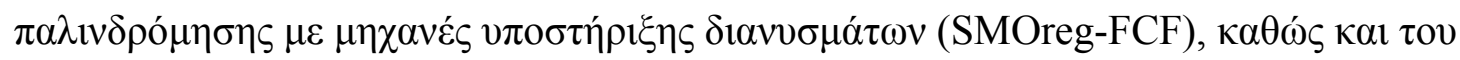

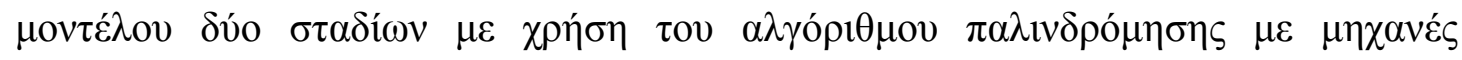

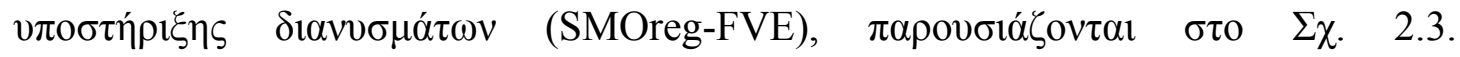

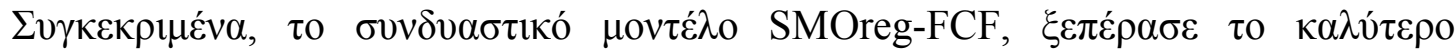




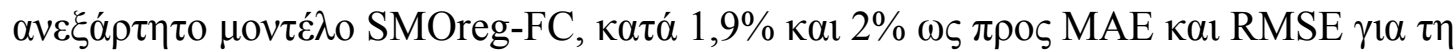

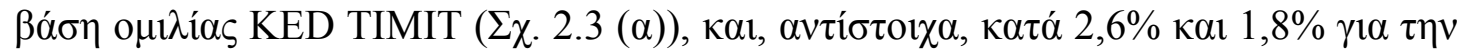

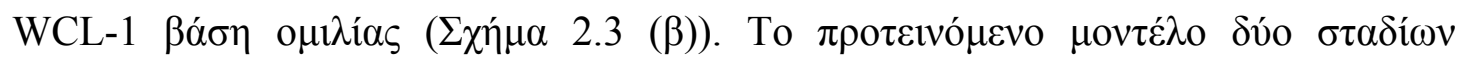

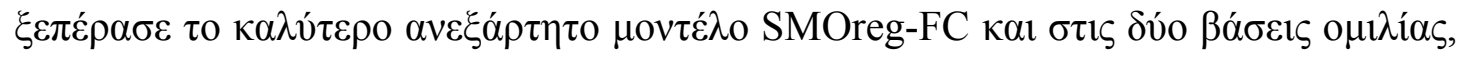

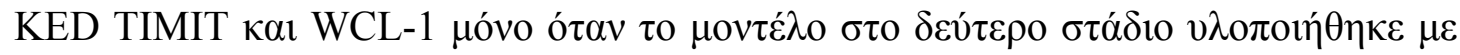

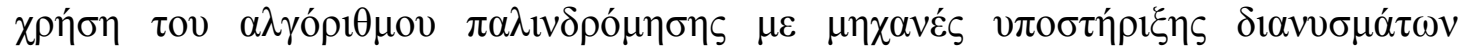

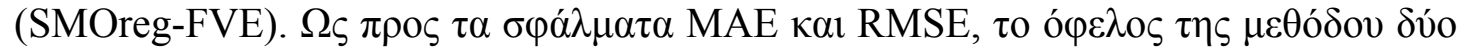

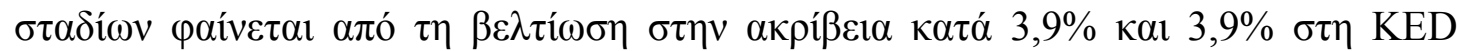

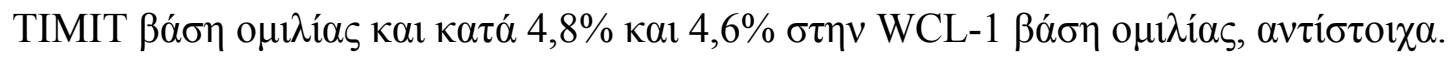

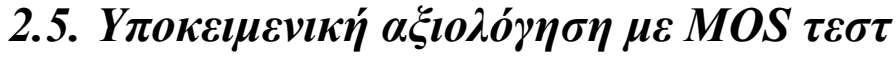

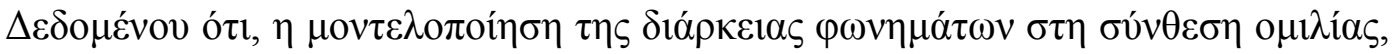

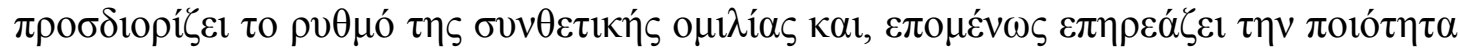

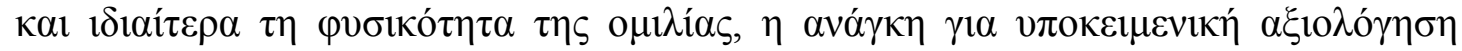

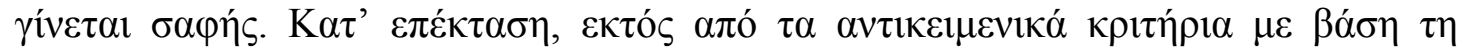

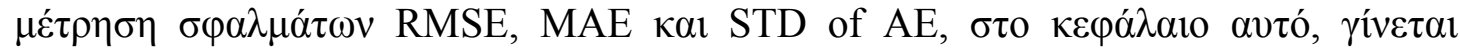

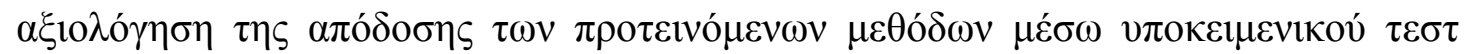
$\pi 010 ́ \tau \eta \tau \alpha \varsigma \tau \eta \varsigma$ o $\mu 1 \lambda i ́ \alpha \varsigma$.

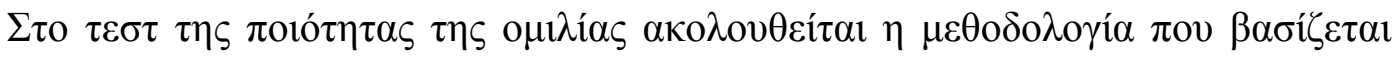

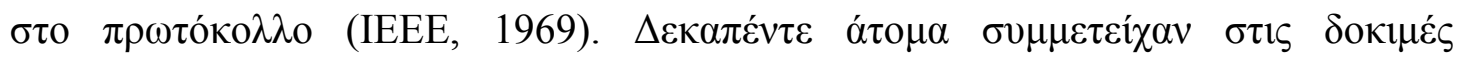

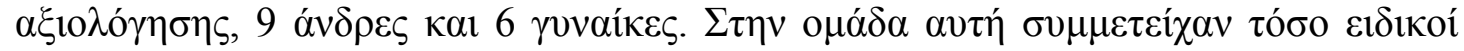

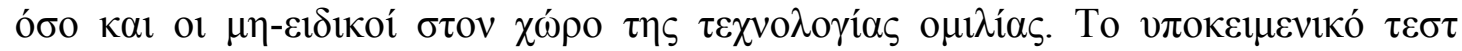

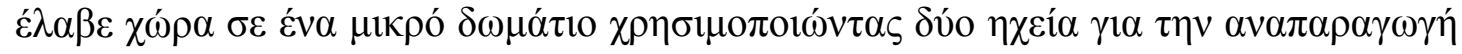

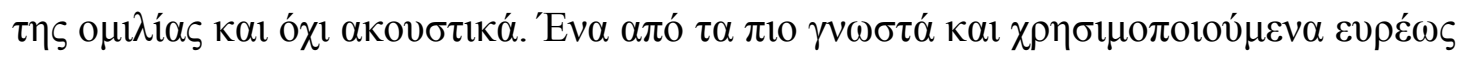

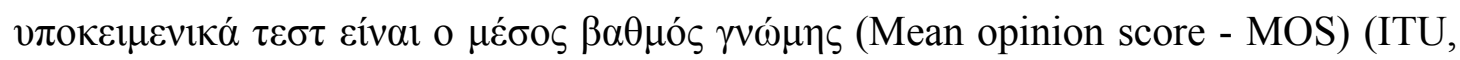

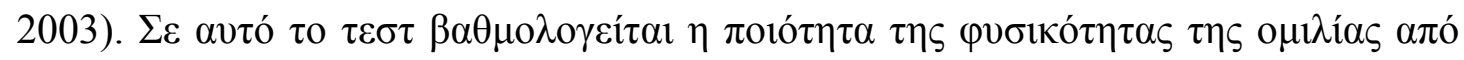

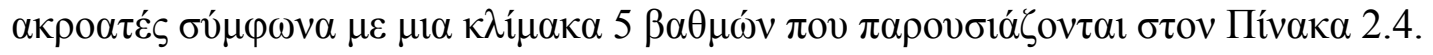




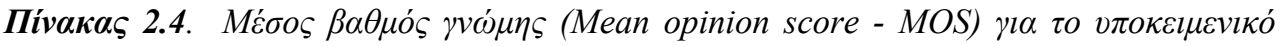
$\tau \varepsilon \sigma \tau$.

\begin{tabular}{|c|c|}
\hline 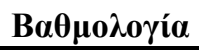 & 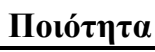 \\
\hline 1 & $\mathrm{Bad}$ \\
\hline 2 & Poor \\
\hline 3 & Fair \\
\hline 4 & Good \\
\hline 5 & Excellent \\
\hline
\end{tabular}

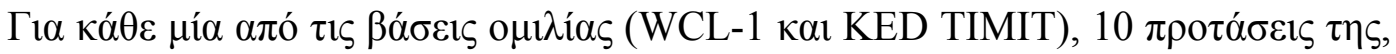

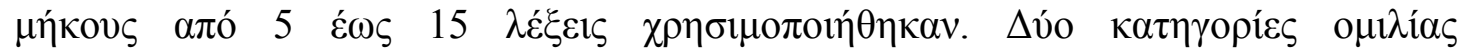

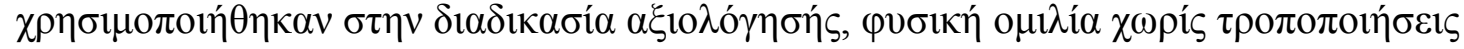

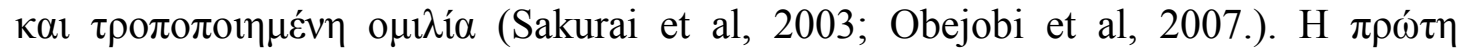

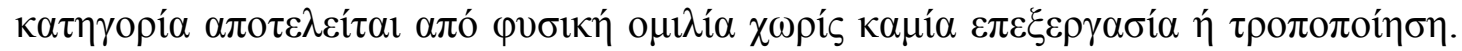

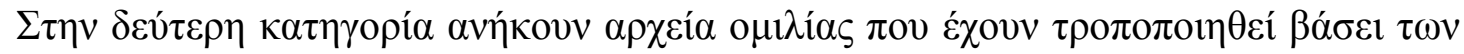

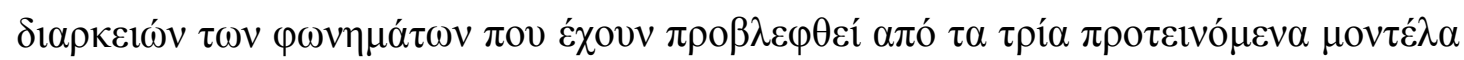

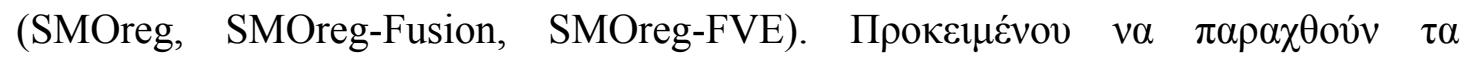

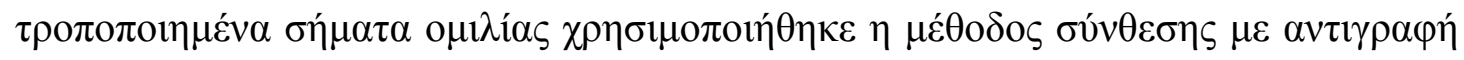
(copy-synthesis) (Laprie $\kappa \alpha 1$ Bonneau, 2007).

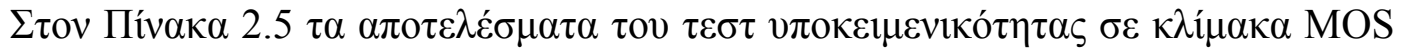

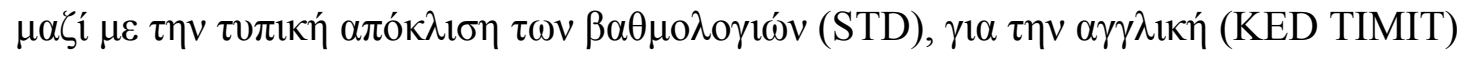

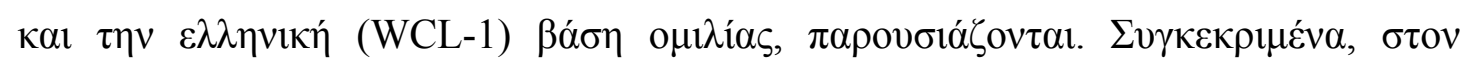

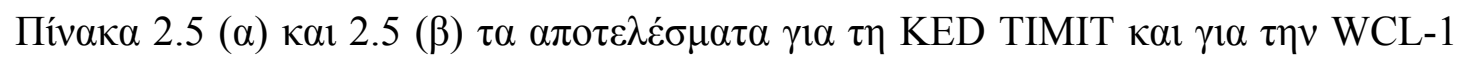

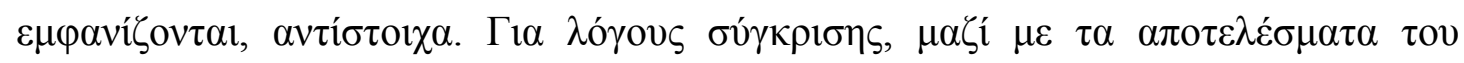

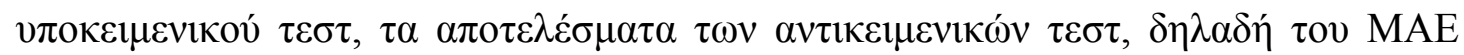

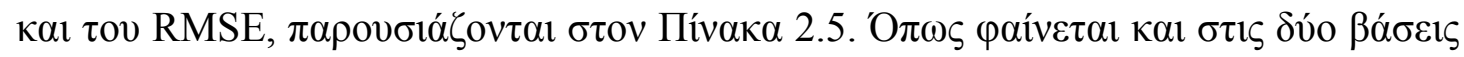

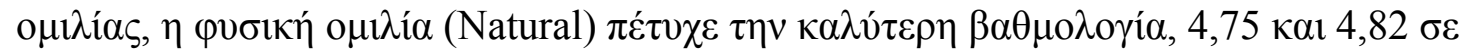

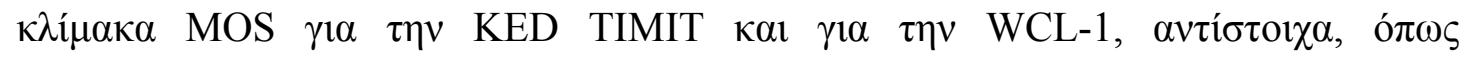

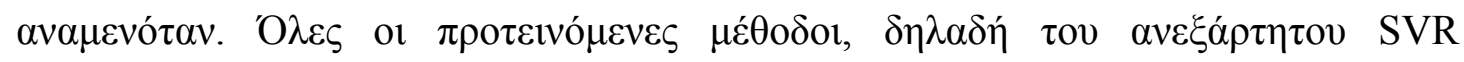

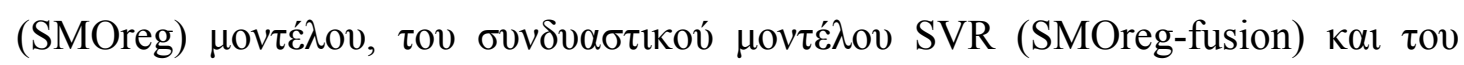

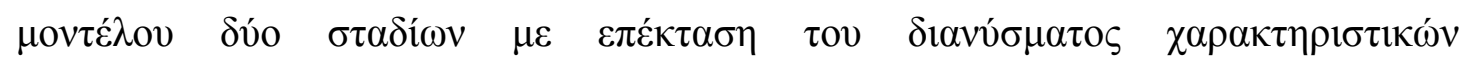

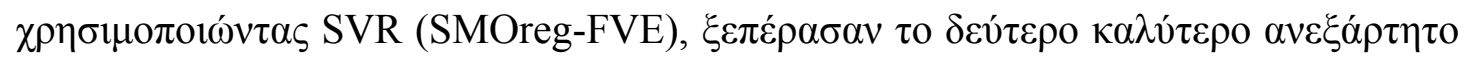

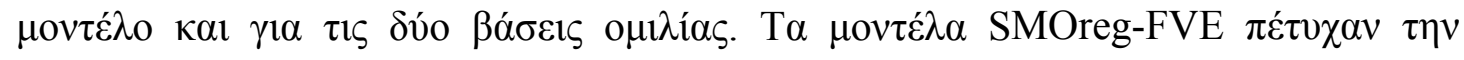

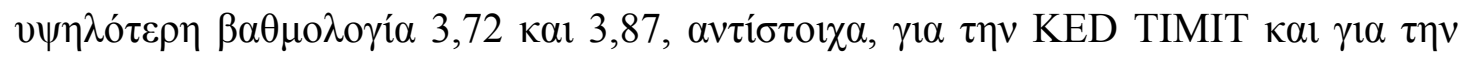

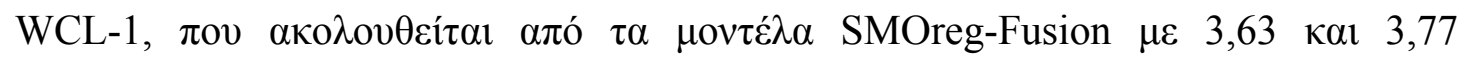




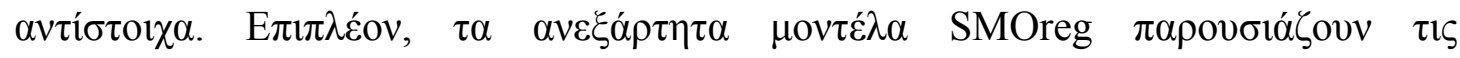

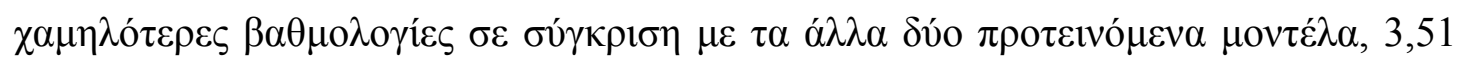

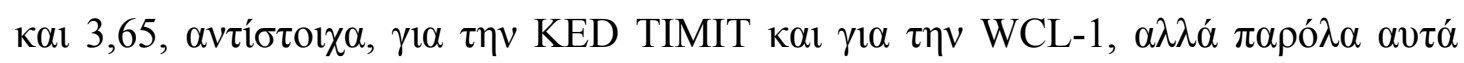

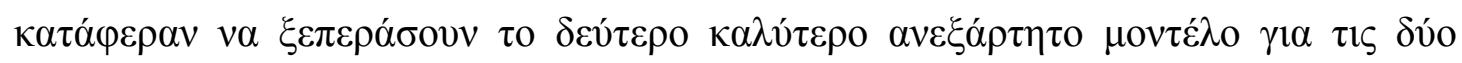

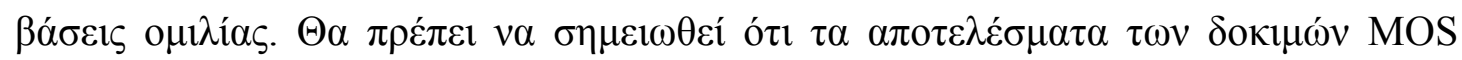

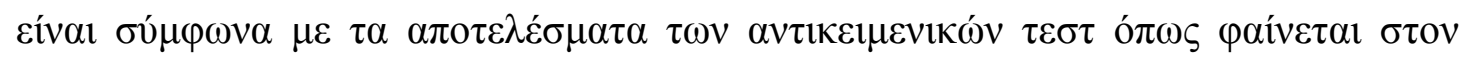
$\pi i ́ v \alpha \kappa \alpha$.

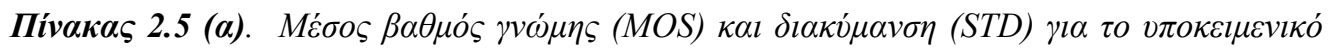

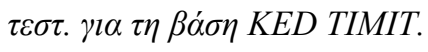

\begin{tabular}{lcccc}
\hline PDMs on the KED TIMIT database & MOS & STD & MAE (ms) & RMSE (ms) \\
\hline Natural & 4.75 & 0.48 & - & - \\
Second-best & 3.3 & 0.83 & 15.82 & 21.35 \\
SMOreg & 3.51 & 0.81 & 14.95 & 20.56 \\
SMOreg-fusion & 3.63 & 0.71 & 14.66 & 20.14 \\
SMOreg-FVE & 3.72 & 0.75 & 14.36 & 19.75 \\
\hline
\end{tabular}

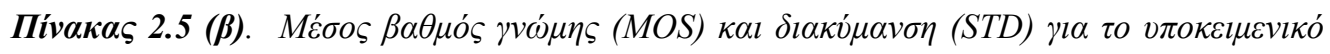
$\tau \varepsilon \sigma \tau . \gamma \iota \alpha \tau \eta \beta \alpha \dot{\alpha} \sigma \eta$.WCL-1.

\begin{tabular}{lcccc}
\hline PDMs on the WCL-1 database & MOS & STD & MAE (ms) & RMSE (ms) \\
\hline Natural & 4.82 & 0.42 & - & - \\
Second-best & 3.41 & 0.84 & 18.00 & 26.19 \\
SMOreg & 3.65 & 0.74 & 16.78 & 25.21 \\
SMOreg-fusion & 3.77 & 0.68 & 16.35 & 24.76 \\
SMOreg-FVE & 3.87 & 0.74 & 15.97 & 24.04 \\
\hline
\end{tabular}

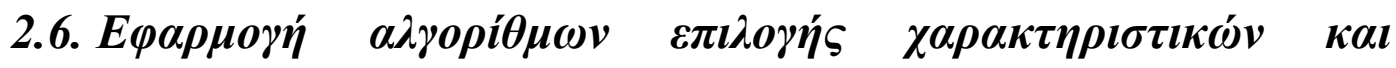

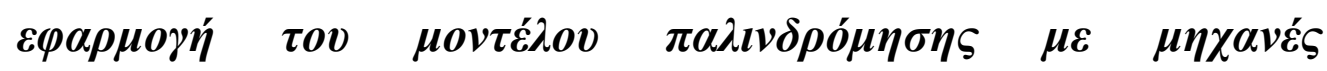

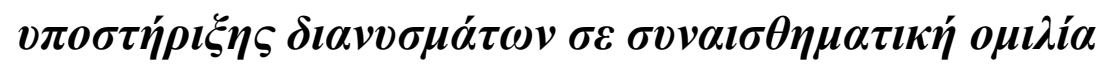

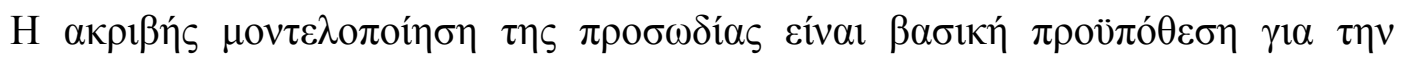

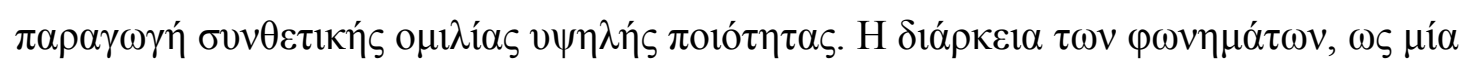

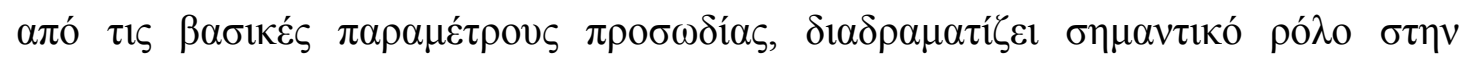

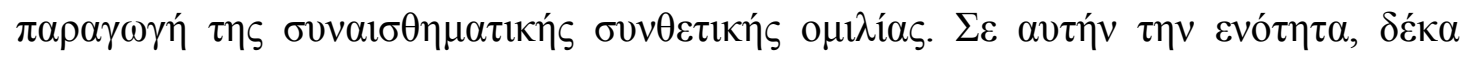

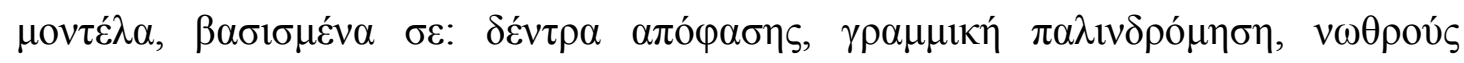

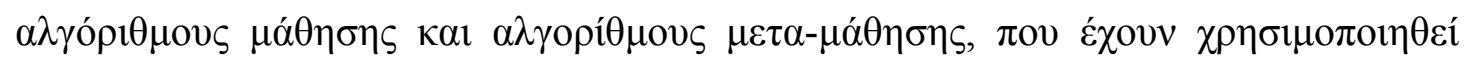




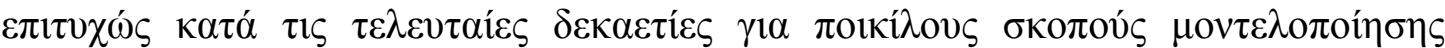

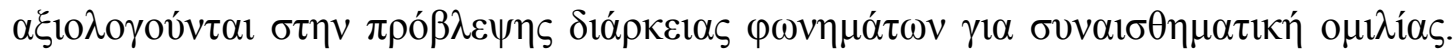

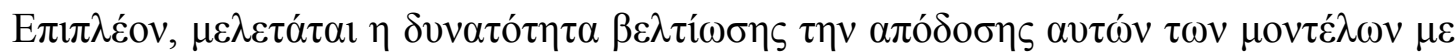

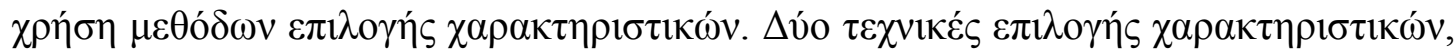

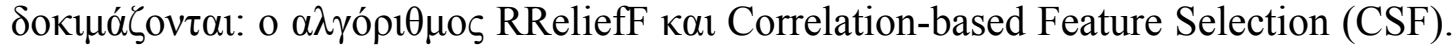

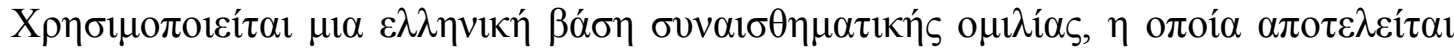

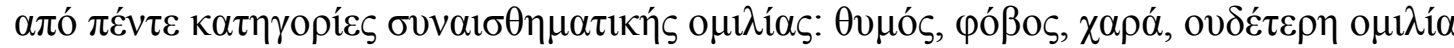

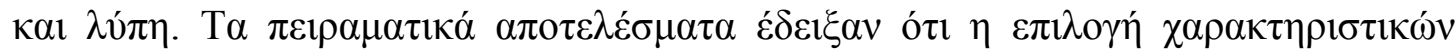

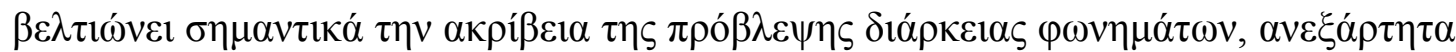

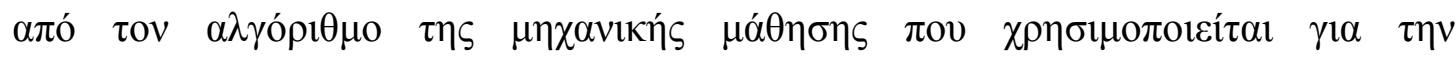

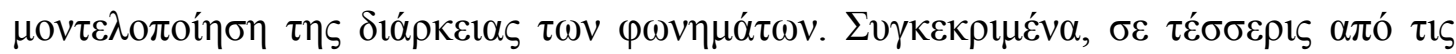

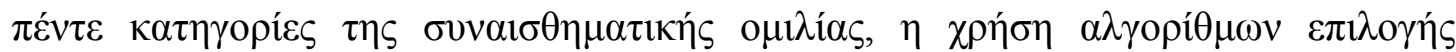

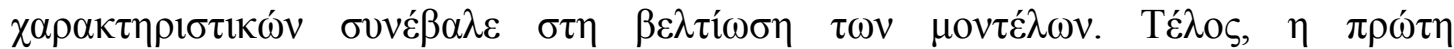

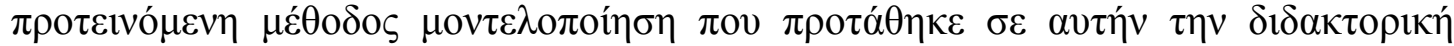

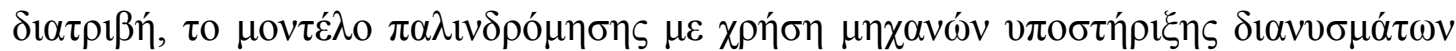

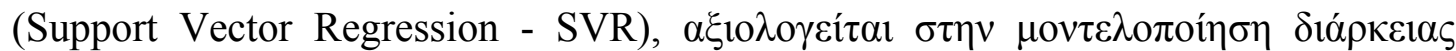

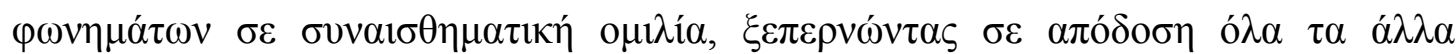
$\mu \circ \tau \tau \varepsilon \dot{\lambda} \lambda$.

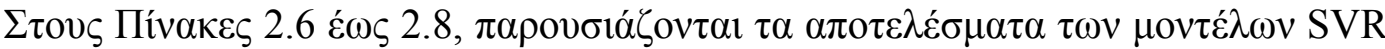

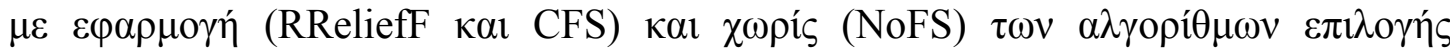

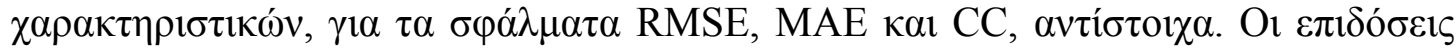

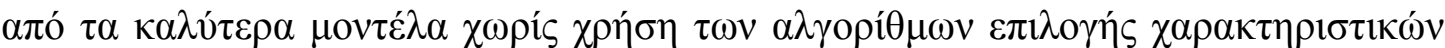

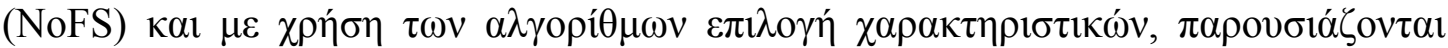

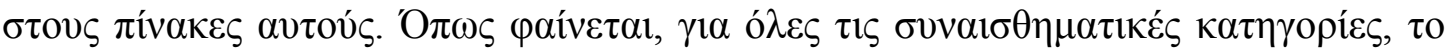

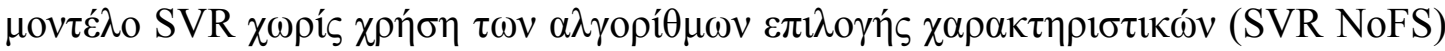

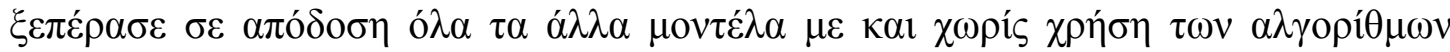

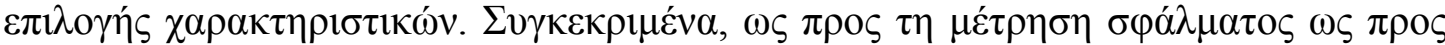

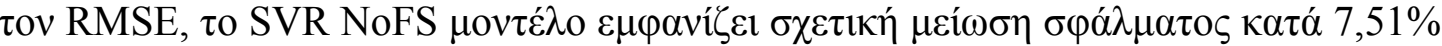

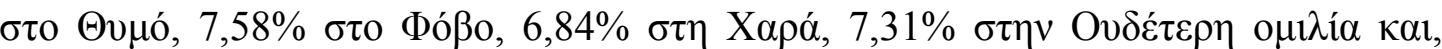
$\tau \varepsilon \dot{\lambda}$

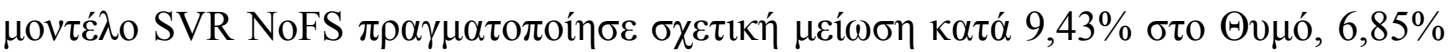

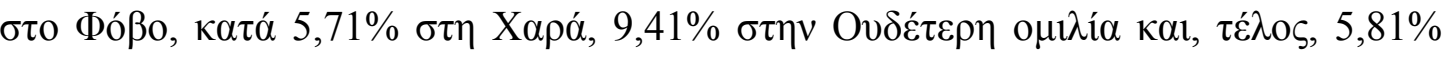

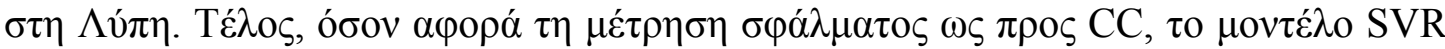




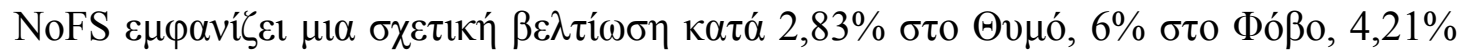

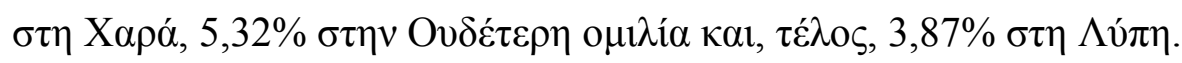

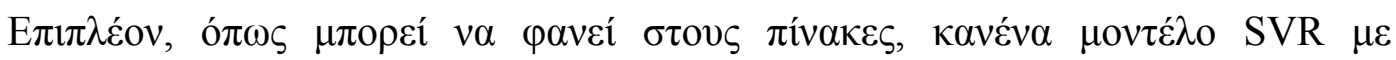

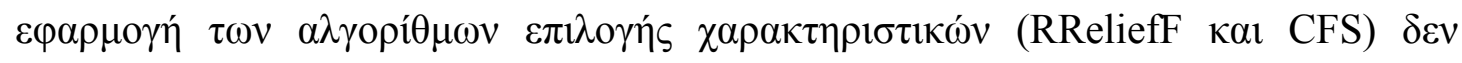

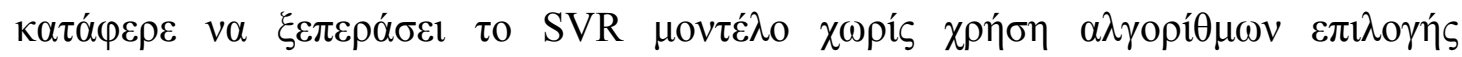

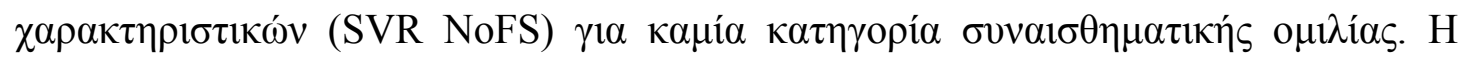

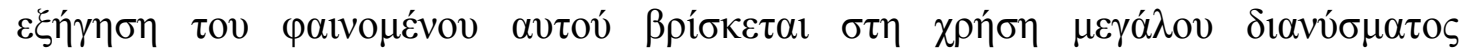

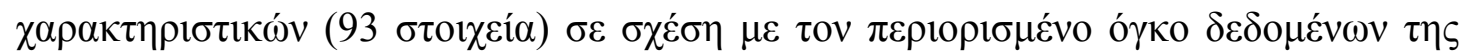

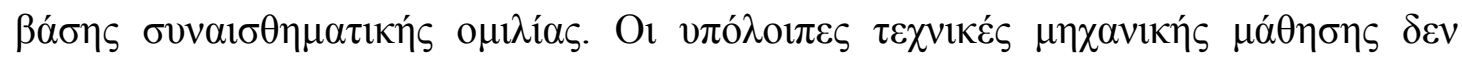

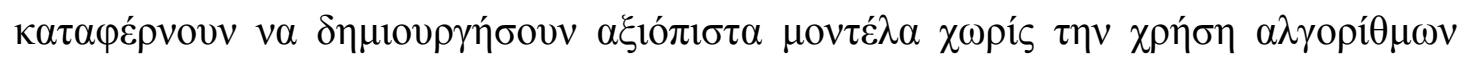

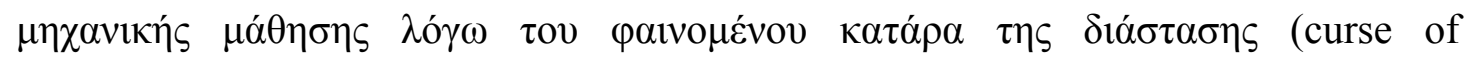

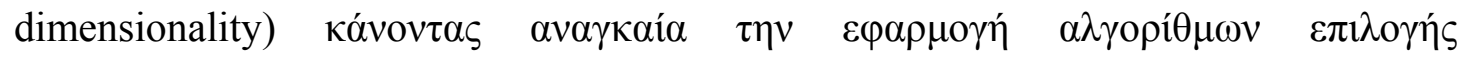

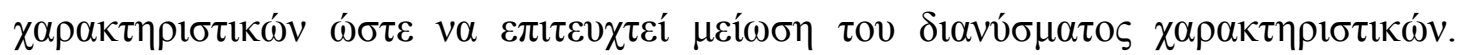

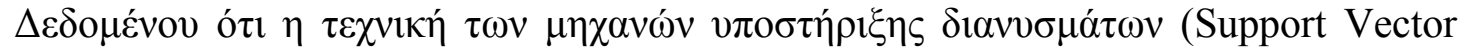

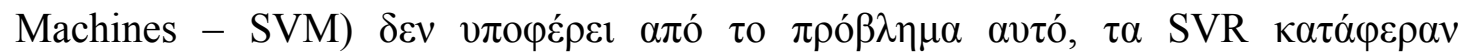

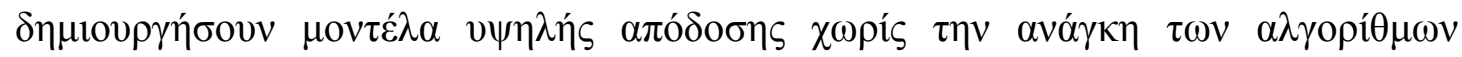

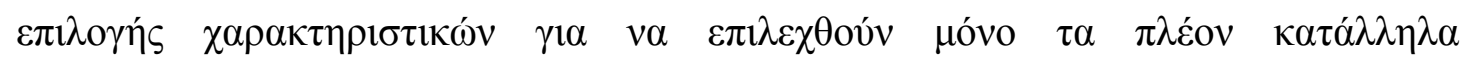

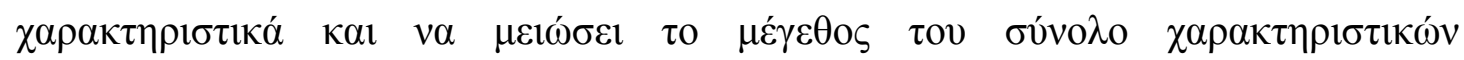
$\gamma \nu \omega \rho \imath \sigma \mu \alpha ́ \tau \omega v$. 


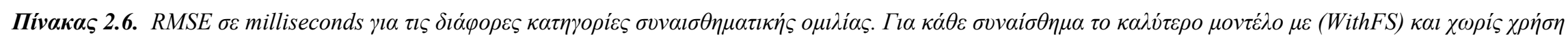

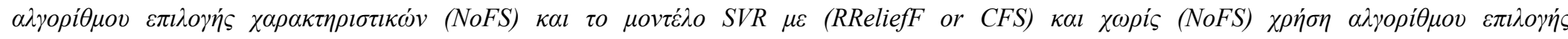

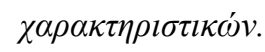

\begin{tabular}{|c|c|c|c|c|c|}
\hline RMSE & 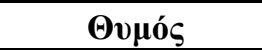 & Фóßos & 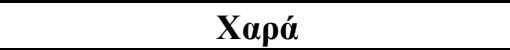 & 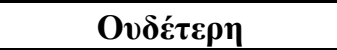 & $\Lambda \hat{\pi} \pi \eta$ \\
\hline Best NoFS & $21.7(\mathrm{~m} 5 \mathrm{p})$ & 20.1 (Add. Regg. m5pR) & 19.0 (Add. Regg. m5pR) & $26.2(\mathrm{~m} 5 \mathrm{p})$ & 20.6 (Add. Regg. m5pR) \\
\hline Best WithFS & $21.3(\mathrm{~m} 5 \mathrm{p}-\mathrm{CFS})$ & 19.8 (m5p - RReliefF) & 19.3 (Add. Regg. m5pR - RreliefF) & 26.0 (m5p - RReliefF) & 20.7 (m5p - CFS) \\
\hline SVR NoFS & 19.7 & 18.3 & 17.7 & 24.1 & 19.1 \\
\hline SVR (RReliefF) & 19.9 & 18.6 & 18.1 & 24.4 & 19.5 \\
\hline$S V R(C F S)$ & 21.2 & 20.0 & 18.8 & 25.8 & 20.9 \\
\hline
\end{tabular}

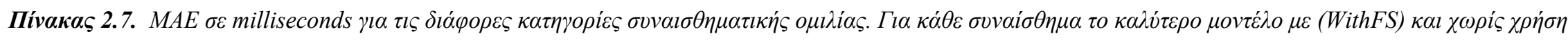

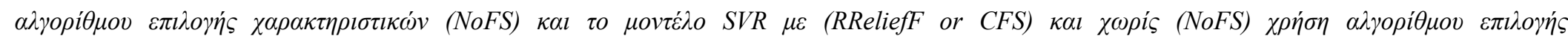

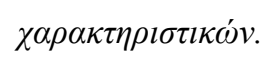

\begin{tabular}{|c|c|c|c|c|c|}
\hline$M A E$ & $\Theta v \mu o ́ \varsigma$ & Фóßos & Xapá & 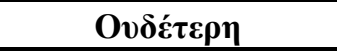 & $\Lambda v ́ \pi \eta$ \\
\hline Best NoFS & $16.1(\mathrm{~m} 5 \mathrm{p})$ & 14.9(Add. Regg. m5pR) & 14.0 (Add. Regg. m5pR) & $17.1(\mathrm{~m} 5 \mathrm{p})$ & 15.6 (Add. Regg. m5p) \\
\hline Best WithFS & $15.9(\mathrm{~m} 5 \mathrm{p}-\mathrm{CFS})$ & 14.6 (m5p - RReliefF) & 14.3 (Add. Regg. m5pR - RReliefF) & $17.0(\mathrm{~m} 5 \mathrm{p}-\mathrm{RReliefF})$ & $15.5(\mathrm{~m} 5 \mathrm{p}-\mathrm{CFS})$ \\
\hline SVR NoFS & 14.4 & 13.6 & 13.2 & 15.4 & 14.6 \\
\hline SVR (RReliefF) & 14.6 & 13.7 & 13.5 & 16.1 & 14.8 \\
\hline$S V R(C F S)$ & 15.5 & 14.8 & 14.2 & 16.8 & 15.5 \\
\hline
\end{tabular}




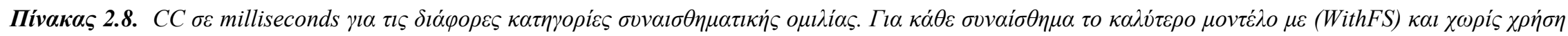

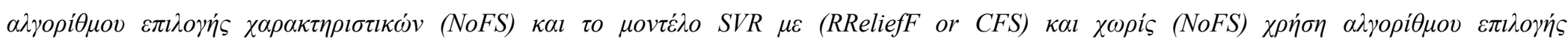

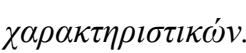

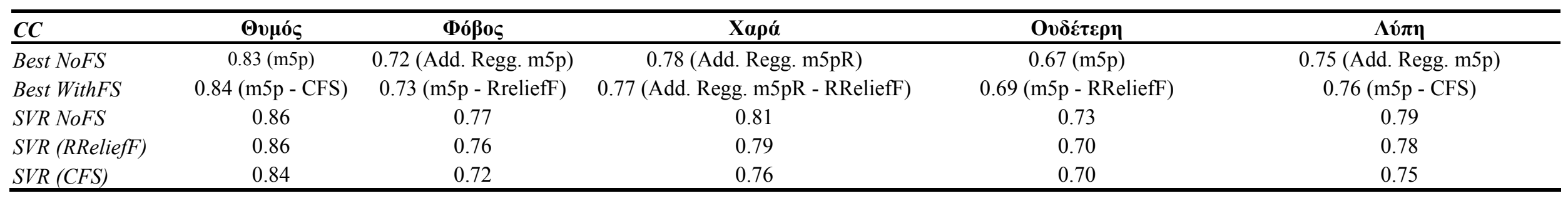




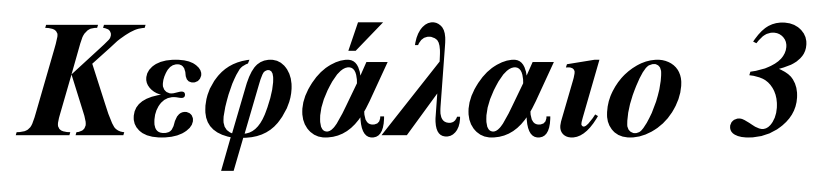

\section{ANA $\Sigma K O \Pi H \Sigma H \quad T H \Sigma \quad \Delta \mathrm{I} \Delta \mathrm{AKTOPIKH \Sigma}$

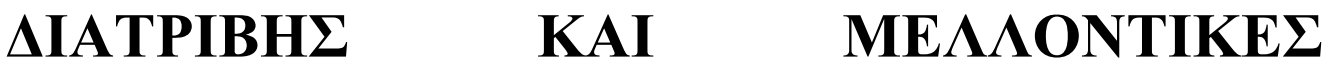

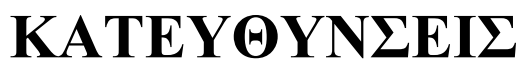

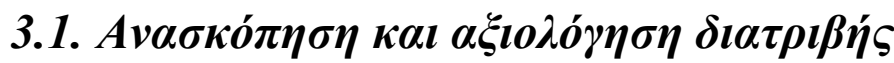

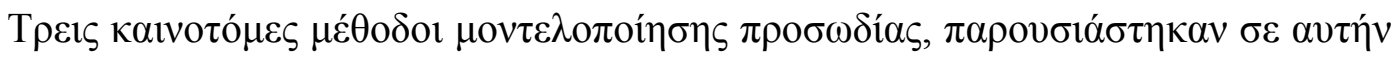

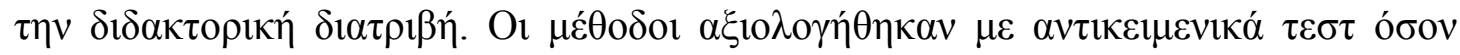

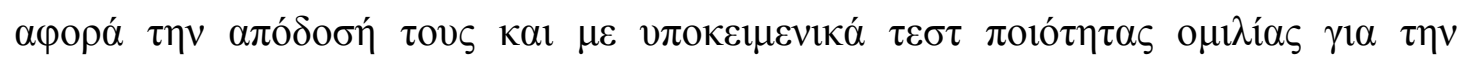

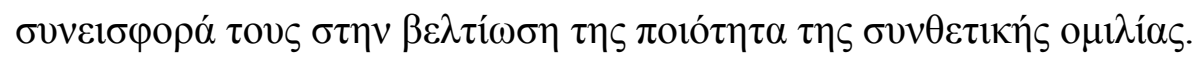

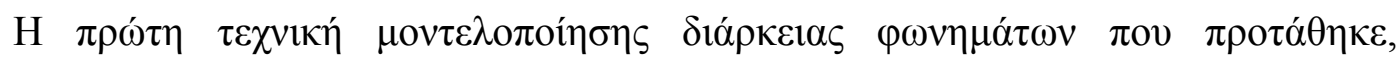

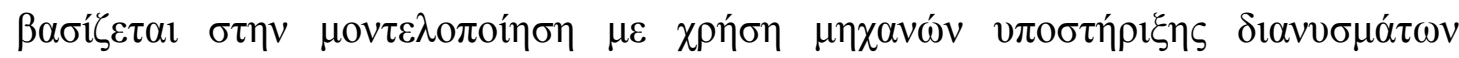

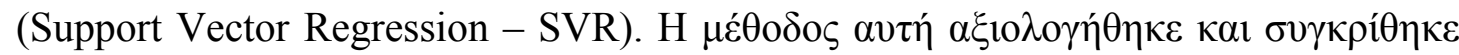

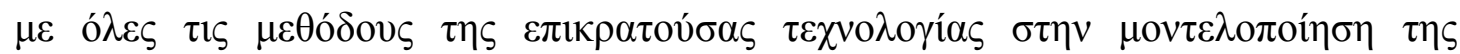

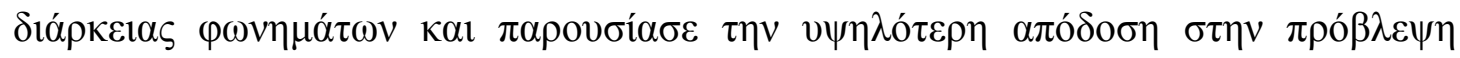

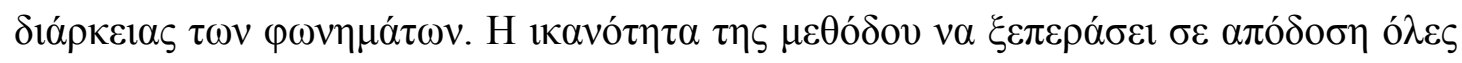

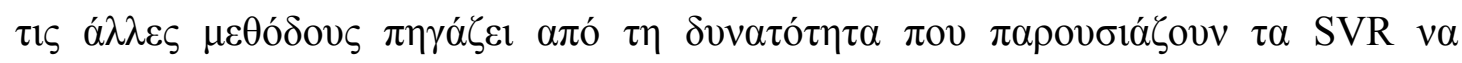

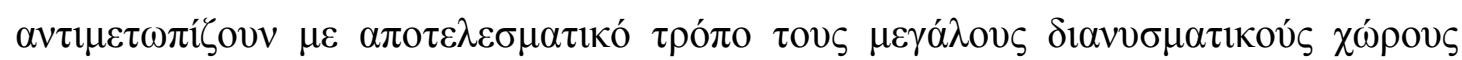

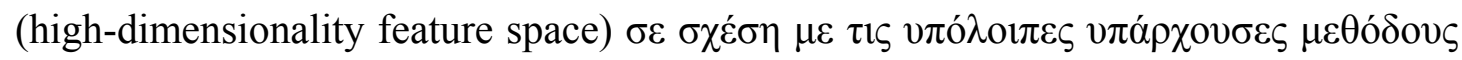

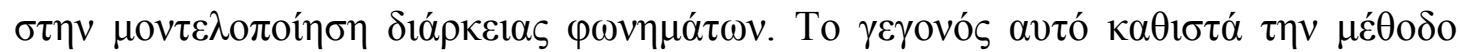

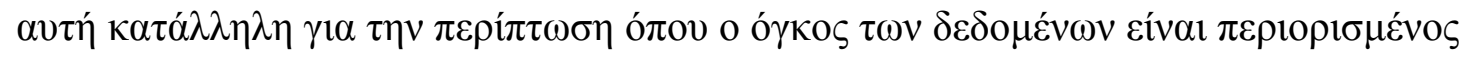




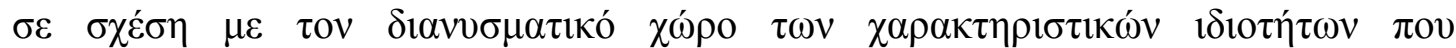

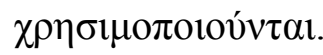

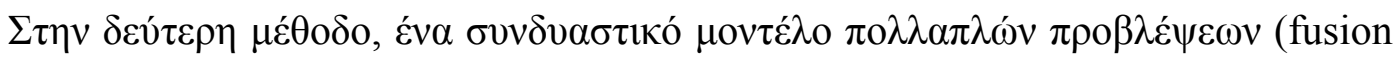

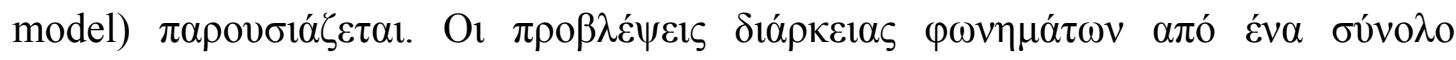

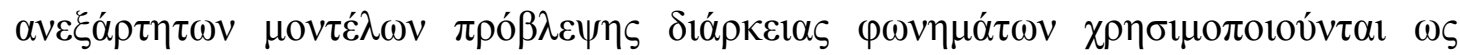

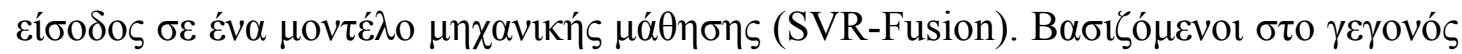

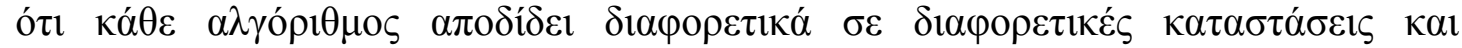

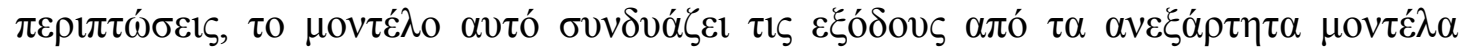

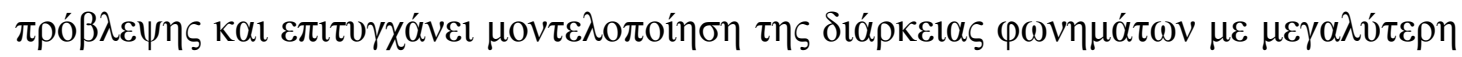

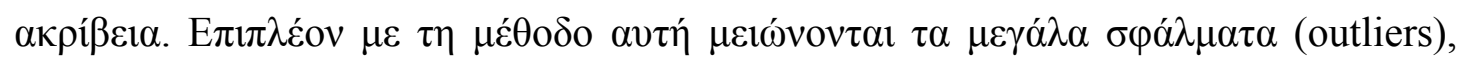

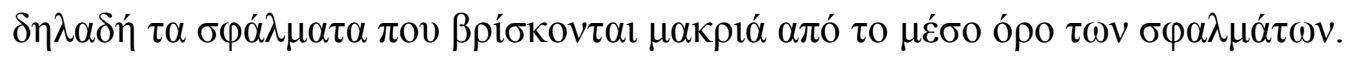

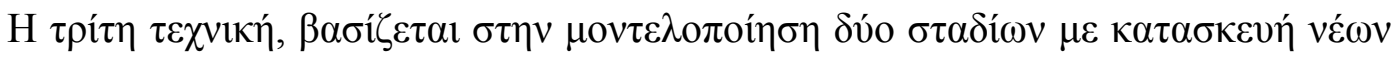

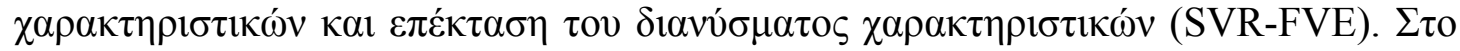

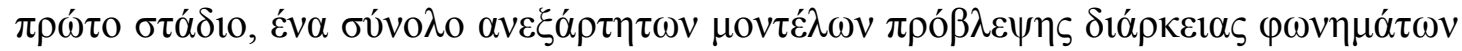

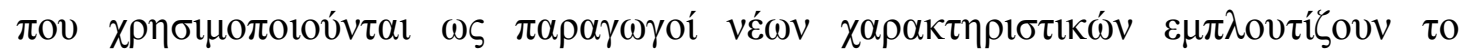

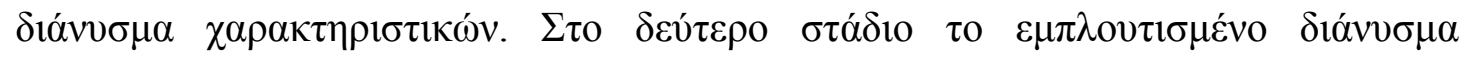

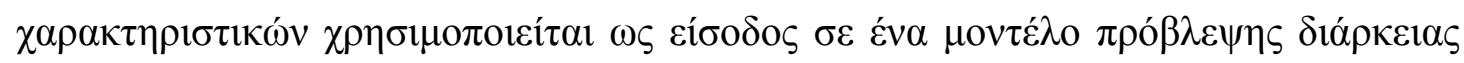

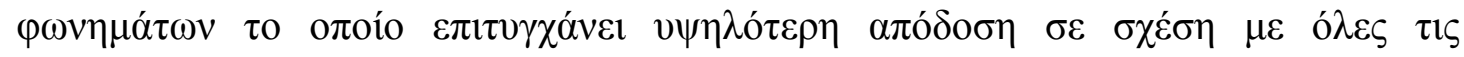

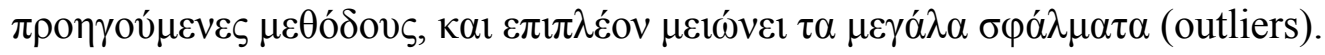

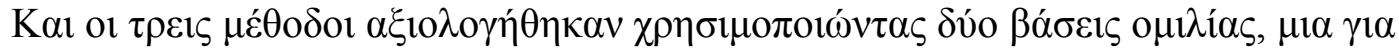

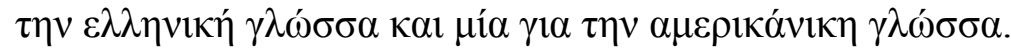

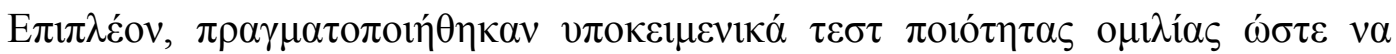

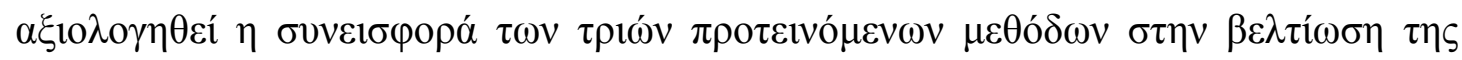

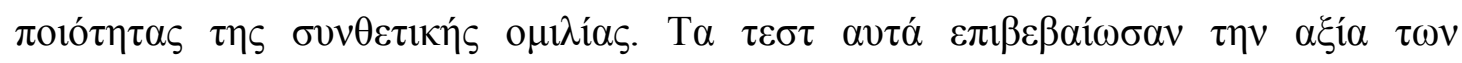

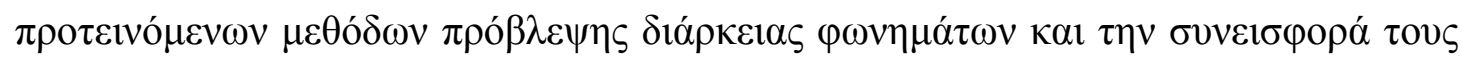

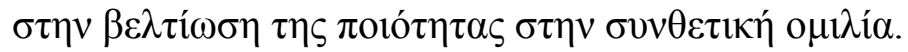

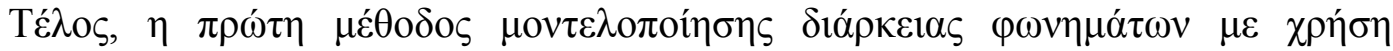

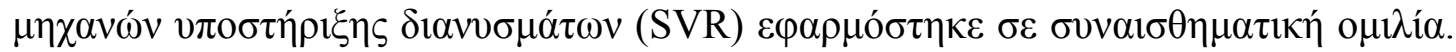

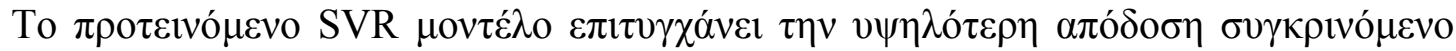

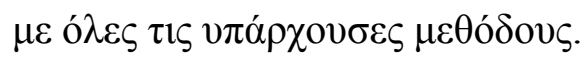




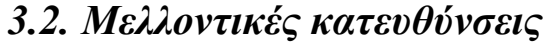

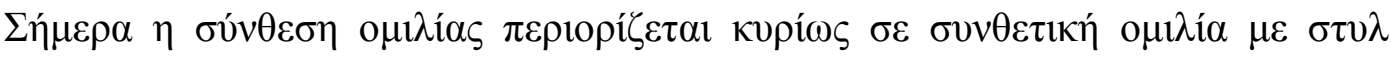

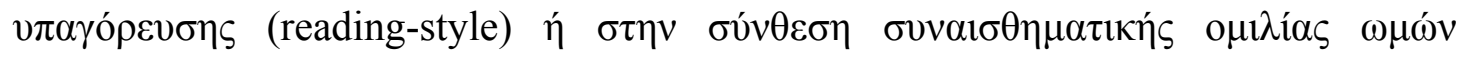

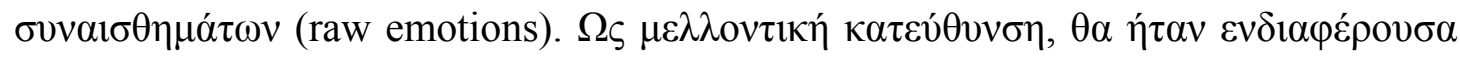

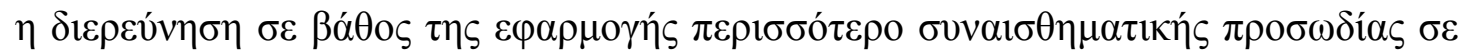

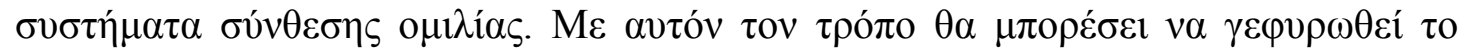

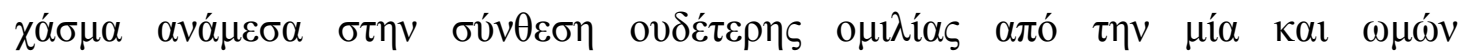

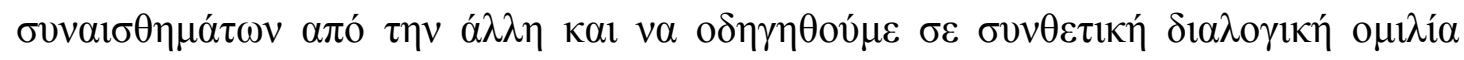

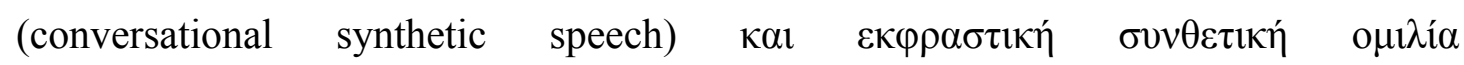

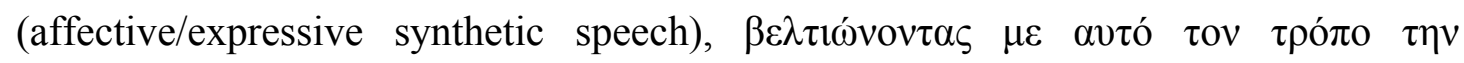

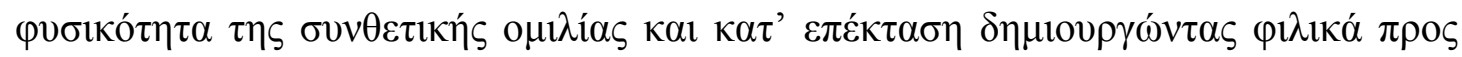

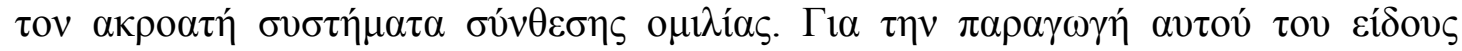

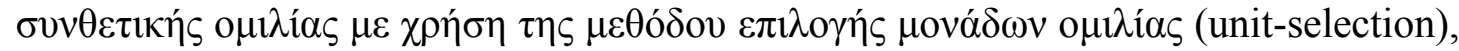

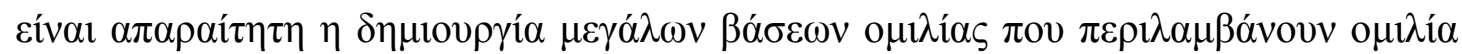

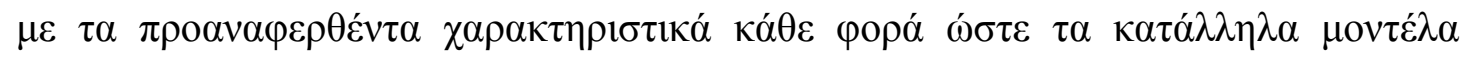

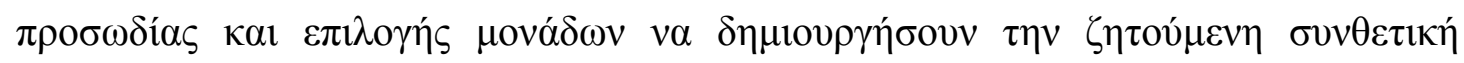

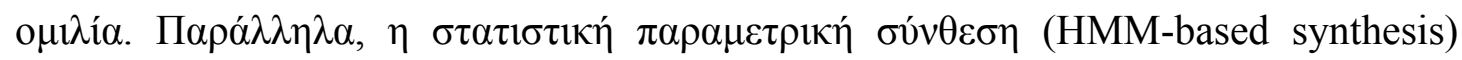

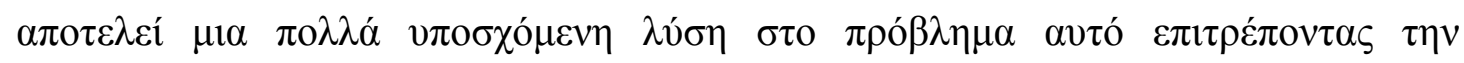

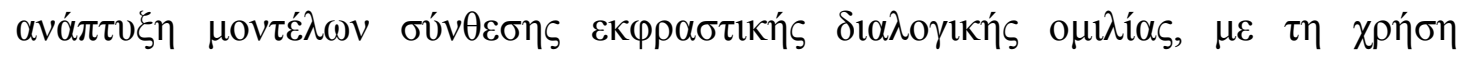

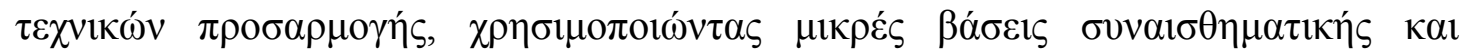

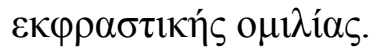




\section{ВIBАIОГРАФІА}

Anastasakos, A., Schwartz, R., Suh, H., 1995. Duration modeling in large vocabulary speech recognition. In Proc. of ICASSP-1995, Detroit, USA, pp. 628-631.

Bartkova, K., Sorin, C., 1987. A model of segmental duration for speech synthesis in French. Speech Communication. 6 (3), 245-260.

Bell, A., Jurafsky, D., Fosler-Lussier, E., Girand, C., Gregory, M., Gildea, D., 2003. Effects of disfluencies, predictability, and utterance position on word form variation in English conversation. Journal of the Acoustical Society of America. 113 (2), 1001-1024.

Bourlard, H., Hermansky, H., Morgan, N., 1996. Towards increasing speech recognition error rates. Speech Communication. 18 (3), 205-231.

Carlson, R., Granstrom, B., 1986. A search for durational rules in real speech database. Phonetica. 43, 140-154.

Chung, H., 2002, Duration models and the perceptual evaluation of spoken Korean. In Proc. of Speech Prosody, France, pp. 219-222.

Clark, J., Yallop, C., 1995. An introduction to phonetics and phonology, second ed. Blackwell, Oxford.

Crystal, T.H., House, A.S., 1988. Segmental durations in connected-speech signals: Current results. Journal of the Acoustical Society of America. 83 (4), 1553-1573.

Dixon, N.R., Maxey, H.D., 1968. Terminal analog synthesis of continuous speech using the diphone method of segment assembly. IEEE Trans. Audio and Electroacoustics. 16 (1), 40-50.

Dutoit, T., 1997. An Introduction to Text-To-Speech Synthesis, Kluwer Academic Publishers, Dordrecht.

Epitropakis, G., Tambakas, D., Fakotakis, N., Kokkinakis, G., 1993. Duration modelling for the Greek language. In Proc. of EUROSPEECH-1993, Berlin, Germany, pp. 1995-1998.

Fukada, T., Tokuda, K., Kobayashi, T., Imai, S., 1992. An adaptive algorithm for melcepstral analysis of speech. In Proc. of ICASSP-1992, California, USA, pp 137140. 
Furui, S., 2000. Digital Speech Processing, Synthesis, and Recognition, second ed., Marcel Dekker, New York.

Gao, B., Qian, Y., Wu, Z., Soong, F.K., 2008. Duration Refinement by Jointly Optimizing State and Longer Unit Likelihood. In Proc. of ICSLP-2008, Brisbane, Australia, pp. 2266-2269.

Goubanova, O., King, S., 2008. Bayesian networks for phone duration prediction. Speech Communication. 50 (4), 301-311.

Gregory, M., Bell, A., Jurafsky, D., Raymond, W., 2001. Frequency and predictability effects on the duration of content words in conversation. Journal of the Acoustical Society of America. 110 (5), 27-38.

Harris, C.M., 1953. A study of the building blocks in speech. Journal of the Acoustic Society of America. 25 (5), 962-969.

Huang, X., Acero, A., Hon, H.W., 2001. Spoken Language Processing: a guide to theory, algorithm, and system development. Prentice Hall, Redmond.

Hunt, A.J.; Black, A.W., 1996. Unit selection in a concatenative speech synthesis system using a large speech database. In Proc. ICASSP-1996, Atlanta, Georgia, pp. 373-376.

IEEE, 1969. Recommended practice for speech quality measurements. IEEE Transactions on Audio and Electroacoustics. 17 (3), 225-246.

ITU-TRecommendation P.800.1, 2003. Mean opinion score (MOS) terminology.

Iwahashi, N., Sagisaka, Y., 2000. Statistical modeling of speech segment duration by constrained tree regression. IEICE Trans. Inform. Systems. E83-D (7), 1550-1559.

Jennequin, N., Gauvain, J.L., 2007. Modeling duration via lattice rescoring. In Proc. of ICASSP-2007, Honolulu, Hawaii, pp. 641-644.

Klatt, D.H., 1976. Linguistic uses of segmental duration in English: Acoustic and perceptual evidence. Journal of the Acoustic Society of America. 59 (5), 12091221.

Kohler, K.J., 1988. Zeistrukturierung in der Sprachsynthese. ITG-Tagung Digitalc Sprachverarbeitung. 6, 165-170.

Krishna, N.S., Murthy, H.A., 2004. Duration modeling of Indian languages Hindi and Telugu. In Proc. of the 5th ISCA Speech Synthesis Workshop, Pittsburgh, USA, pp. 197-202. 
Laprie, Y., Bonneau, A., 2007. Construction of perception stimuli with copy synthesis. In Proc. of ICPhS-2007, Sarrebruck, Allemagne, pp. 2189-2192.

Latorre, J., Buchholz, S., Akamine, M., 2010. Usages of an external duration model for HMM-based speech synthesis. In Proc. of Speech Prosody 2010, Chicago, USA.

Laver, J., 1980. The phonetic description of voice quality. Cambridge University Press, Cambridge.

Laver, J., 1994. Principles of Phonetics. Cambridge University Press, Cambridge.

Lazaridis, A., Zervas, P., Kokkinakis, G., 2007. Segmental duration modeling for Greek speech synthesis. In Proc. of IEEE ICTAI-2007, Patras, Greece, pp. 518521.

Lazaridis, A., Mporas, I., Ganchev, T., Kokkinakis, G., Fakotakis, N., 2011. Improving Phone Duration Modeling using Support Vector Regression Fusion. Speech Communication. 53 (1), 85-97.

Lee, S., Oh, Y.H., 1999a. Tree-based modeling of prosodic phrasing and segmental duration for Korean tts systems. Speech Communication. 28 (4), 283-300.

Lee, S., Oh, Y.H., 1999b. CART-based modelling of Korean segmental duration. In Proc. of Oriental COCOSDA-1999, Taipei, Taiwan, pp. 109-112.

Levinson, S., 1986. Continuously variable duration hidden Markov models for speech analysis. In Proc. of ICASSP-1986, Tokyo, Japan, pp.1241-1244.

Mitchell, C., Harper, M., Jamieson, L., Helzermam, R., 1995. A parallel implementation of a hidden Markov model with duration modeling for speech recognition. Digital Signal Processing. 5, 43-57.

Möbius, B., van Santen, J., 1996. Modeling segmental duration in German text-toSpeech synthesis. In Proc. of ICSLP-1996, Philadelphia, USA pp. 2395-2398.

Odejobi, O.A., Wong, S.S., Beaumont, A.J., 2007. A fuzzy decision tree-based duration model for Standard Yoruba text-to-speech synthesis. Computer Speech and Language. 21 (2), 325-349.

Pols, L.C.W., Wang, X., ten Bosch, L.F.M., 1996. Modelling of phone duration (using the TIMIT database) and its potential benefit for ASR. Speech Communication. 19 (2), 161-176. 
Pylkkonen, J., Kurimo, M., 2004. Duration modeling techniques for continuous speech recognition. In Proc. of ICSLP-2004, Jeju Island, Korea, pp. 385-388.

Riley, M., 1992. Tree-based modelling for speech synthesis. In: Bailly, G., Benoit, C., Sawallis, T.R. (Eds.), Talking Machines: Theories, Models and Designs. Elsevier, Amsterdam, pp. 265-273.

Sakurai, A., Hirose, K., Minematsu, N., 2003. Data-driven generation of f0 contours using a superpositional model. Speech Communication. 40 (4), 535-549.

Schweitzer, A., Möbius, B., 2004. Exemplar-based production of prosody: Evidence from segment and syllable durations, In Proc. of Speech Prosody 2004, Nara, Japan, pp. 459-462

Shannon, R.V., Zeng, F.G., Kamath, V., Wygonski, J., Ekelid, M., 1995. Speech recognition with primarily temporal cues. Science. 13 (5234), 303-304.

Silén, H., Helander, E., Nurminen, J., Gabbouj, M., 2010. Analysis of Duration Prediction Accuracy in HMM-Based Speech Synthesis. In Proc. of Speech Prosody, Chicago, USA.

Simoes, A.R.M., 1990. Predicting sound segment duration in connected speech: An acoustical study of Brazilian Portugese. In Proc. of Workshop on Speech Synthesis, Autrans, France, pp.173-176.

Takeda, K., Sagisaka, Y., Kuwabara, H., 1989. On sentence-level factors governing segmental duration in Japanese. Journal of Acoustic Society of America. 86 (6), 2081-2087.

Teixeira, J.P., Freitas, D., 2003. Segmental durations predicted with a neural network. In Proc. of the European Conference on Speech Communication and Technology, Geneva, Switzerland, September, pp. 169-172.

Tokuda, K., Yoshimura, T., Masuko, T., Kobayashi, T., Kitamura, T., 2000. Speech parameter generation algorithm for HMM-based speech synthesis. In Proc. of ICASSP-2000, Istanbul, Turkey, pp 1315-1318.

van Santen, J.P.H., 1994. Assignment of segmental duration in text-to-speech synthesis. Computer Speech \& Language. 8 (2), 95-128.

van Santen, J.P.H., 1998. Timing. In: Sproat, R. (Eds.), Multilingual Text-to- Speech Synthesis - The Bell Labs Approach. Springer, New York, pp. 115-139.

Vapnik, V., 1995. The Nature of Statistical Learning Theory. Springer, New York. 
Vapnik, V., 1998. Statistical Learning Theory. Wiley, New York.

W3C, 1999. W3C, Standard Generalized Markup Language: http://www.w3.org/MarkUp/SGML/

W3C, 2003. W3C, Speech Synthesis Markup Language Version 1.0: http://www.w3.org/TR/2003/CR-speech-synthesis-20031218/

Yoshimura, T., Tokuda, K., Masuko, T., Kobayashi, T., Kitamura, T., 1998. Duration Modeling in HMM-based Speech Synthesis System. In Proc. of ICSLP-1998, Sydney, Australia, pp.29-32.

Yoshimura, T., Tokuda, K., Masuko, T., Kobayashi, T., Kitamura, T., 1999. Simultaneous modeling of spectrum, pitch and duration in HMM-based speech synthesis. In Proc. of EUROSPEECH-1999, Budapest, Hungary, pp 2347-2350.

Zen, H., Masuko, T., Yoshimura, T., Tokuda, K., Kobayashi, T., Kitamura, T., 2007a. State duration modeling for HMM-based speech synthesis. IEICE Trans. on Inf. \& Syst. E90-D (3), 692-693.

Zen, H., Tokuda, K., Masuko, T., Kobayashi, T., Kitamura, T., 2004. Hidden SemiMarkov Model Based Speech Synthesis. In Proc. of ICSLP-2004, Jeju Island, Korea, pp. 1393-1396. 